\author{
Universidade de São Paulo \\ Instituto de Física
}

\title{
Supercordas e Aspectos da Correspondência AdS/CFT
}

\author{
Renann Lipinski Jusinskas \\ ORIENTADOR: Prof. Dr. Victor de Oliveira Rivelles
}

Dissertação de mestrado apresentada ao Instituto de Física para a obtenção do título de Mestre em Ciências.

\section{Banca Examinadora:}

Prof. Dr. Victor de Oliveira Rivelles (Orientador - IFUSP)

Prof. Dr. Fernando Tadeu Caldeira Brandt (IFUSP)

Prof. Dr. Ricardo Iván Medina Bascur (UNIFEI) 


\section{FICHA CATALOGRÁFICA}

\section{Preparada pelo Serviço de Biblioteca e Informação}

do Instituto de Física da Universidade de São Paulo

Jusinskas, Renann Lipinski

Supercordas e aspectos da correspondência AdS/CFT São Paulo, 2010.

Dissertação (Mestrado) - Universidade de São Paulo. Instituto de Física, Departamento de Física Matemática.

Orientador: Prof. Dr. Victor de Oliveira Rivelles.

Área de Concentração: Física.

Unitermos: 1. Supergravidade; 2. Supersimetria;

3. Teoria de cordas; 4. Correspondência AdS/CFT. 


\section{Sumário}

$\begin{array}{ll}\text { Agradecimentos } & \text { v }\end{array}$

$\begin{array}{ll}\text { Prólogo } & \text { vi }\end{array}$

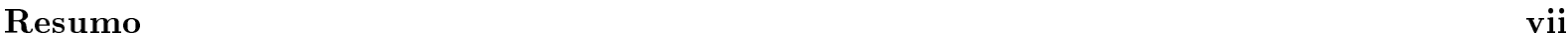

$\begin{array}{lc}\text { Abstract } & \text { viii }\end{array}$

1 Introdução $\quad 1$

2 Corda Bosônica $\quad 3$

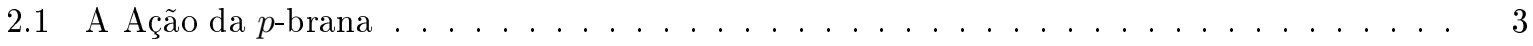

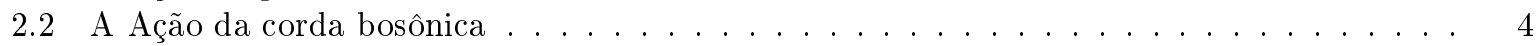

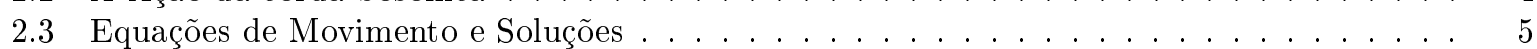

2.4 Massa e Energia da Corda . . . . . . . . . . . . . . . . . . . . . . 7

2.5 O Calibre do Cone de Luz . . . . . . . . . . . . . . . . . . . . . . 8

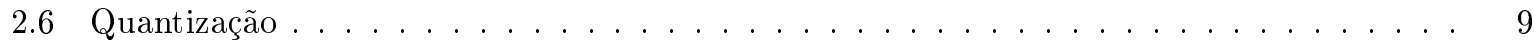

2.6.1 Espectro da Corda Aberta . . . . . . . . . . . . . . . . . . 10

2.6.2 Espectro da Corda Fechada . . . . . . . . . . . . . . . . . . . . . 11

2.7 Dualidade T e D-branas . . . . . . . . . . . . . . . . . . . . . . . 12

2.7.1 Compactificação para Cordas Fechadas . . . . . . . . . . . . . . . . . . . . 12

2.7.2 Compactificação para Cordas Abertas . . . . . . . . . . . . . . . . . . 13

2.8 Simetria de Calibre $U(N) \ldots \ldots \ldots \ldots \ldots \ldots \ldots \ldots \ldots$

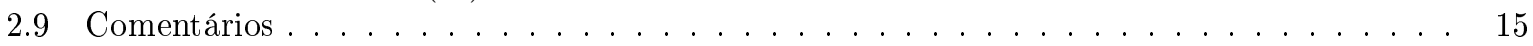

3 Supercorda RNS $\quad 17$

3.1 Supersimetria Local e a Ação RNS . . . . . . . . . . . . . . . . . . . . . . . . . . . . 17

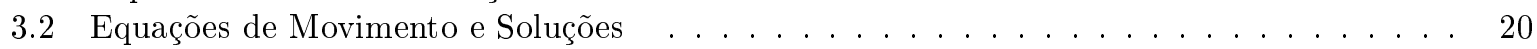

3.3 Cargas Conservadas e Geradores de Super Virasoro . . . . . . . . . . . . . . . . . . . . . 22

3.4 Quantização Canônica Covariante . . . . . . . . . . . . . . . . . . . . 23

3.5 O Calibre do Cone de Luz . . . . . . . . . . . . . . . . . . . . . . . . . 26

3.6 Análise do Espectro de Massa . . . . . . . . . . . . . . . . . . . . . . . 27

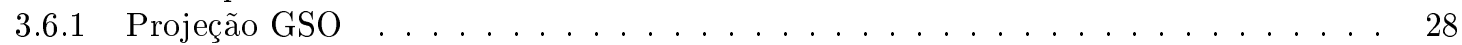

3.6 .2 Espectro da Corda Aberta . . . . . . . . . . . . . . . . . . . . 28

3.6.3 Espectro da Corda Fechada . . . . . . . . . . . . . . . . . . . . . . . . . 29

3.6.4 Supersimetria no Espaço-Tempo? . . . . . . . . . . . . . . . . . 30

3.7 Comentários . . . . . . . . . . . . . . . . . . . . . . . 31 
4 Supercorda GS $\quad 33$

4.1 Supersimetria no Espaço-Tempo . . . . . . . . . . . . . . . . . . . . . . . 33

4.2 Simetria kapa e a Ação GS . . . . . . . . . . . . . . . . . . . . . . . . . . . . . . . . . . . . 34

4.3 Cargas Conservadas e Geradores de Simetrias do Espaço-Tempo . . . . . . . . . . . . . . 37

4.4 Equações de Movimento e Graus de Liberdade . . . . . . . . . . . . . . . . . . . . . . . . . . . . . . . . . . . . . .

4.5 O Calibre do Cone de Luz no Formalismo GS . . . . . . . . . . . . . . . . . . . . . . . 39

4.6 Quantização e Espectro Fundamental . . . . . . . . . . . . . . . . . . . . . . . 41

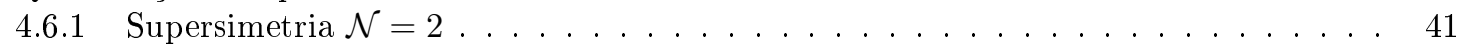

4.6.1.1 Cordas Fechadas Tipo $I I-\mathrm{A} \ldots \ldots \ldots \ldots$. . . . . . . . . . . . . . . . . . . . . . . . .

4.6.1.2 Cordas Fechadas Tipo $I I$-B . . . . . . . . . . . . . . . . 43

4.6.2 Supersimetria $\mathcal{N}=1 \ldots \ldots \ldots \ldots \ldots \ldots$

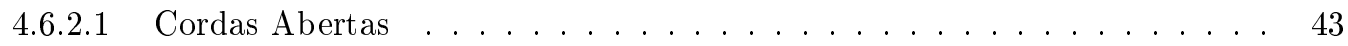

4.6.2.2 Cordas Fechadas Não Orientadas . . . . . . . . . . . . . . . . 44

4.7 Dualidade $\mathrm{T}$ e as Supercordas . . . . . . . . . . . . . . . . . . . . . . . . . . . . . . . . . . . . . . . . . . . .

4.7 .1 Cordas Fechadas . . . . . . . . . . . . . . . . . . . . 45

4.7 .2 Cordas Abertas . . . . . . . . . . . . . . . . . . . . 46

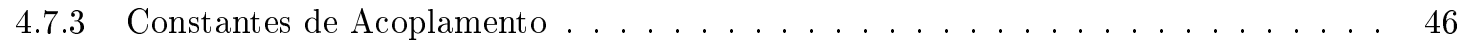

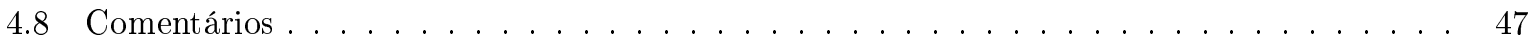

5 Branas e Supergravidade $\quad 49$

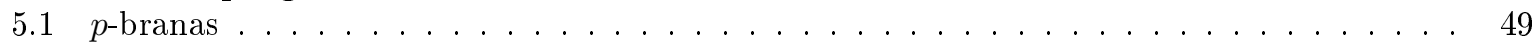

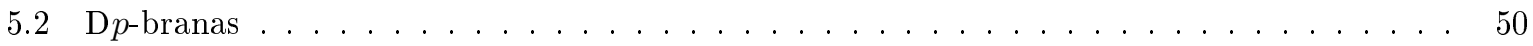

5.2.1 Supersimetria e Simetria kapa do Campo de Calibre . . . . . . . . . . . 51

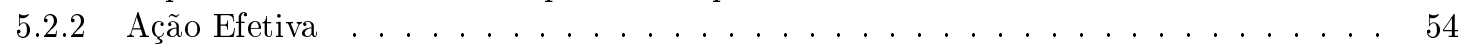

5.3 Ações da Supergravidade . . . . . . . . . . . . . . . . . . . . . . 56

5.3 .1 Teoria $M$ e Supergravidade $D=11 \ldots \ldots \ldots \ldots \ldots$

5.3 .2 Supergravidade $I I-\mathrm{A} \ldots \ldots \ldots \ldots \ldots \ldots \ldots$

5.3 .3 Supergravidade $I I-\mathrm{B} \ldots \ldots \ldots \ldots \ldots \ldots \ldots$

5.4 Comentários . . . . . . . . . . . . . . . . . . . . . 60

6 Aspectos da Correspondência AdS/CFT $\quad 61$

$6.1 \mathcal{N}=4$ Super Yang-Mills . . . . . . . . . . . . . . . . . . . . . . 61

6.1 .1 O Conteúdo de Campos e a Ação . . . . . . . . . . . . . . . . . . . . . . 61 . . . . . . . . . . .

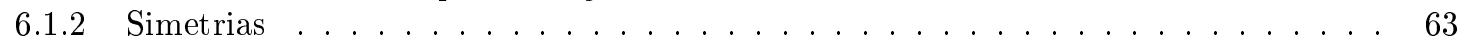

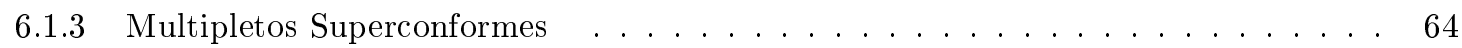

6.1 .4 Expansão $\frac{1}{N} \ldots \ldots \ldots \ldots \ldots \ldots \ldots$

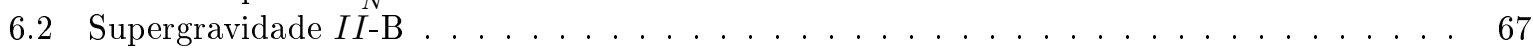

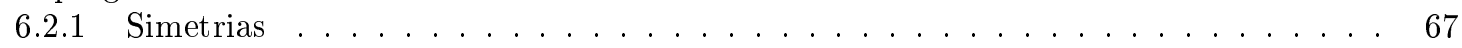

6.2.2 Equações de Movimento e Soluções Extremas . . . . . . . . . . . . . . . . . . 68

6.2.3 A 3-brana e a Geometria $A d S_{5} \times S^{5} \ldots \ldots \ldots \ldots$. . . . . . . . . . 71

6.3 A Conjectura de Maldacena e o Dicionário da Correspondência . . . . . . . . . . . . . 73

6.3.1 Algumas Comparações . . . . . . . . . . . . . . . . . . . . . . . . 73

6.3.2 A Conjectura e o Dicionário . . . . . . . . . . . . . . . . . . 74

6.4 Comentários . . . . . . . . . . . . . . . . . . . . 76

$\begin{array}{llr}7 & \text { Conclusão } & \mathbf{7 7}\end{array}$

$\begin{array}{lr}\text { A A Dimensão do Espaço-Tempo } & 79\end{array}$ 
B Algumas Propriedades de Espinores em Várias Dimensões 85

B.1 Matrizes $\Gamma \ldots \ldots \ldots \ldots \ldots \ldots \ldots \ldots$

B.2 Espinores de Majorana, Weyl e Majorana-Weyl . . . . . . . . . . . . . . . . . . . . 86

B.3 Propriedade Especial . . . . . . . . . . . . . . . . . . . . . . . . . . 89

C Supersimetria e Representações $\quad 91$

C.1 Introdução . . . . . . . . . . . . . . . . . . . . . . . . . . . . . . . 91

C.2 Supersimetria em $D=2$ e a Supercorda RNS . . . . . . . . . . . . . . . . . . . . . . . . . . . . .

C.3 Supersimetria em Dimensões Arbitrárias . . . . . . . . . . . . . . . . . . . . 93

C.3.1 Supersimetria em $D=4$ e suas Representações . . . . . . . . . . . . . . . . 94

C.3.1.1 Representações Não Massivas . . . . . . . . . . . . . . . 95

C.3.1.2 Representações Massivas e o vínculo BPS . . . . . . . . . . . . . . . 95

C.3.2 Supersimetria e Supergravidade em $D=10,11 \ldots \ldots$. . . . . . . . . . 96

D Matrizes Singulares e a Simetria kapa 99

D.1 Matrizes Singulares . . . . . . . . . . . . . . . . . . . . . . . . 99

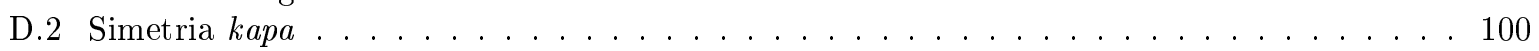

$\begin{array}{lr}\text { E Simetria Conforme } & 101\end{array}$

$\begin{array}{lr}\text { Referências Bibliográficas } & 103\end{array}$ 


\section{Agradecimentos}

Agradeço, em primeiro lugar, à minha família. À minha mãe, Inês, sempre tão atenciosa e carinhosa, e que, mesmo nos momentos mais difíceis, soube me ouvir e confortar com seu amor. Ao meu pai, Luiz, agradeço esse mesmo amor que, mesmo contido, me orientou e aconselhou sabiamente, com palavras que nem sempre gostei de ouvir, mas precisamente as que eu necessitava entender. À minha irmã, Francine, que, junto aos meus pais, sempre me apoiou em todas as decisões e me ajudou a ver o mundo como eu o vejo hoje e entender um pouco mais as pessoas.

Agradeço aos meus professores que, de uma forma ou de outra, fazem parte do que sou como físico. Em especial, agradeço aos meus ex-orientadores Gilberto Medeiros Kremer e Miguel Abbate, verdadeiros exemplos tanto como pessoas quanto como cientistas, cada um a seu modo, mas ambos fundamentais. Lamento profundamente ter me afastado todo esse tempo mas conto com sua compreensão e amizade.

Agradeço aos meus colegas e amigos da sala 307, Leandro e Pedro, idealizadores do CFT, por inúmeras experiências, discussões, brincadeiras, desabafos, idéias... Por ouvirem meus devaneios e ajudarem nos ajustes finos da dissertaçao, com comentários e sugestões de grande valor. Eu espero sinceramente podermos fazer a diferença e contribuir de alguma forma para a ciência.

Agradeço aos meus colegas da pós-graduação na USP e a todos que contribuíram de forma menos direta, por proporcionarem um ambiente tão favorável, sempre dispostos a discutir e compartilhar experiências.

Agradeço aos meus revisores ortográficos. Com pequenas ou grandes contribuições, definitivamente tornaram o texto mais apresentável. Um agradecimento especial vai para a Carol que, mesmo diante de um texto avançado, se dispôs a ler e consertar minhas falhas.

Por fim, agradeço ao Prof. Dr. Victor de Oliveira Rivelles pela orientação e ao CNPq pelo apoio financeiro. 


\section{Prólogo}

Tomo este espaço para alguns comentários pessoais em relação ao texto da dissertação e algumas das idéias nela contidas.

Apesar de ser um trabalho de revisão, eu acredito na contribuição que o texto pode oferecer, mesmo para os mais experientes que eu no assunto. Tentei escrevê-lo como gostaria que tivessem escrito para mim. São frequentes, na vasta bibliografia de livros e artigos sobre o assunto, comentários como "é fácil ver que...". Há no texto passagens, demonstrações rápidas e simples (algumas nem tanto) de idéias que, talvez triviais, talvez irrelevantes, são omitidas nas várias referências. Concordo que uma vez que os conceitos tenham sido entendidos, fica tudo mais fácil. Eu mesmo espero não ter abusado de comentários desse nível ao longo do texto. Nesse caso, tomo para mim toda a responsabilidade por omissões e possíveis encadeamentos confusos, ou até errados, de ideias. Além disso, todas as palavras expressam meu amadurecimento ao longo desses dois anos. Tentei, não sei se bem sucedido, abordar o assunto com uma das ferramentas mais bem desenvolvidas pelos físicos, a análise de simetrias. Espero deixar claro que tal abordagem não é original, apenas tentei repassá-la de forma mais amigável. Tenho muito a aprender.

Meu outro comentário é mais filosófico, envolvendo o modo como encaro a teoria de cordas e seu potencial de descrição da natureza, como uma teoria de grande unificação.

Quando decidi por esse caminho certamente ouvi críticas. Mais preocupantes, entretanto, eram suas origens. Meus próprios mentores dizendo que isso era um desperdício do meu potencial. Ouvi, e ainda ouço, comentários de desprezo, críticas ferozes de pessoas que não conhecem a metade de tudo o que aprendi até aqui sobre o assunto (o que é ainda muito pouco). Perguntas naturais como "Onde foram parar as outras 6?"' são recorrentes no meu dia-a-dia e geralmente apontam pontos críticos da teoria. É evidente que tais questionamentos são fundamentais e certamente a teoria está incompleta. Entretanto, é importante que a arrogância do ceticismo não subjugue a ousadia da curiosidade e da descoberta: a riqueza matemática e física da teoria de cordas é impressionante (esta última por vezes ignorada mas sempre presente nas idéias dos verdadeiros cientistas). Deixando de lado a leviandade da maioria das críticas (e dos críticos) afirmo aqui que não me arrependo das escolhas que fiz. Conforme um grande pesquisador disse certa vez, mesmo que a teoria de cordas esteja ainda longe de uma descrição satisfatória da natureza, ela pode conter os ingredientes da possível e tão almejada teoria fundamental, e isso já é razão suficiente para estudá-la. E, afinal, se a teoria for considerada inconsistente, apesar de sua beleza, teremos como saldo um enorme desenvolvimento da matemática e, certamente, uma indicação de qual direção seguir, mesmo que seja apenas uma placa dizendo "Não Entre!".

${ }^{1}$ Para entendê-la, basta dar uma olhada na página 27. 


\section{Resumo}

Este é um trabalho de revisão. Os principais formalismos no desenvolvimento da teoria de cordas são introduzidos e discutidos: a corda bosônica e as supercordas no formalismo de Ramond-Neveu-Schwarz e no formalismo de Green-Schwarz. São introduzidas também as açôes efetivas no limite de baixas energias das D-branas (ação DBI) e das teorias de supergravidade $(D=10,11)$. Por fim, são analisados alguns aspectos da correspondência AdS/CFT, enunciando a conjectura e introduzindo algumas das entradas do dicionário. Os apêndices contêm tópicos de grande relevância para o texto e podem ajudar a esclarecer vários raciocínios e passagens ao longo do mesmo. 


\section{Abstract}

This is a review work. The main formalisms on the development of string theory are introduced and discussed: the bosonic string and the superstrings formalisms of Ramond-Never-Schwarz and GreenSchwarz. The effective actions in the low energy limit of the D-branes (DBI action) and supergravity theories $(D=10,11)$ are introduced as well. Finally, some aspects of the AdS/CFT correspondence are analised, enunciating the conjecture and introducing some entries of the dictionary. The appendices contains some topics that are highly relevant for the whole text and may help clarify several ideas and arguments through it. 


\section{Capítulo 1}

\section{Introdução}

A teoria de cordas é, provavelmente, umas das teorias que mais oscilou entre o esquecimento e a glória em toda a história da física teórica. Com mais de 40 anos de seus rudimentos, a dúvida, e mesmo a descrença, estão presentes na comunidade científica.

Tendo sido introduzida na década de 60, seu objetivo inicial era descrever forças nucleares. Nesse sentido, estas seriam vistas como objetos unidimensionais que ligariam os constituintes da matéria. Seu potencial, entretanto, foi reconhecido logo em seguida, uma vez identificado em seu espectro um estado associado ao gráviton, a partícula mensageira das forças gravitacionais.

Nesse contexto nasceu também a supersimetria. Havia a necessidade de incorporar uma descrição fermiônica na teoria, que continha apenas bósons. Assim, possuindo em seu espectro cordas abertas e fechadas, entre bósons e férmions, a teoria ganhou destaque como uma possível unificação das forças da natureza.

Após em certo período de estagnação, que incluiu o desenvolvimento da supergravidade, a teoria passou pelo que é chamado hoje a Primeira Revolução das Cordas. Em um breve período, houve grandes mudanças e desenvolvimentos. O principal deles, associado a um cancelamento de anomalias nas teorias do tipo $\mathrm{I}^{1},[1]$.

Durante o seu desenvolvimento a teoria passou a contar com cinco tipos diferentes de descrição, o que, como esperado de uma teoria de unificação, é bastante desagradável. Em um grande trabalho de revisão, E. Witten conseguiu conectá-las em uma série de dualidades, apontando a existência de uma possível teoria em 11 dimensões que daria origem à todas as descrições conhecidas das cordas, a teoria M. Essa ficou conhecida como a Segunda Revolução das Cordas.

Nesse meio tempo, um grande trabalho de J. Polchinski, [2], levou os teóricos de cordas a considerarem seriamente a existência de objetos p-dimensionais, as D-branas, que são hoje de fundamental importância na área.

Seguindo a evolução da teoria, no final da década de 90, J. Maldacena introduziu a correspondência AdS/CFT e, em termos práticos, fundou um novo ramo de pesquisa e impulsionou a teoria de cordas, trazendo-a, uma vez mais, para a vanguarda da física teórica e promovendo uma aproximação entre várias áreas de pesquisa.

O objetivo deste texto é introduzir os formalismos mais tradicionais da teoria de cordas e descrever introdutoriamente a conjectura de Maldacena.

No capítulo 2, a teoria de cordas é motivada por uma generalização da ação da partícula relativística, com um estudo de suas propriedades e particularidades. A teoria quântica é obtida com a escolha do calibre do cone de luz e o espectro de estados da corda aberta e da corda fechada é analisado. Discute-se

\footnotetext{
${ }^{1}$ Nomenclatura a ser esclarecida durante o texto.
} 
também a dualidade $\mathrm{T}$, inferindo a existência das D-branas.

Em seguida, no capítulo 3, a ação da corda bosônica é generalizada de modo a incluir a supersimetria local na folha-mundo. A quantização da teoria é feita covariantemente pelo procedimento canônico e também no calibre do cone de luz. A partir de uma análise do espectro, a supersimetria no espaço-tempo é sugerida, sendo introduzida no capítulo 4, com o formalismo de Green-Schwarz das supercordas. Neste ponto, a extensão da ação bosônica não é evidente e a simetria kapa é introduzida para balancear os graus de liberdade bosônicos e fermiônicos. O espectro da teoria é obtido no calibre do cone de luz e discutido extensivamente, analisando, inclusive, os efeitos da dualidade T no setor fermiônico.

O capítulo 5 introduz as ações efetivas no limite de baixas energias associadas a teoria de supercordas. A ação de Dirac-Born-Infeld é introduzida, obtendo as transformações de supersimetria e simetria kapa do campo de calibre da D-brana. As ações da supergravidade são introduzidas, com uma rápida motivação da teoria M.

Por fim, o capítulo 6 apresenta alguns aspectos da correspondência AdS/CFT. A teoria de $\mathcal{N}=4$ Super Yang-Mills é introduzida e discutida. A supergravidade $I I$-B é desenvolvida, com uma abordagem de suas soluções extremas, em especial o caso da 3-brana. O enunciado da Conjectura de Maldacena é apresentado em seus vários níveis de intensidade junto a algumas entradas do dicionário da correspondência.

Os apêndices constituem um material muito relevante para o texto. O apêndice A apresenta o famoso cálculo da dimensão crítica do espaço-tempo para as cordas bosônicas. O apêndice B discute prorpiedades de espinores em várias dimensões, especialmente aquelas associadas aos bilineares presentes nas teorias supersimétricas. A supersimetria é apresentada introdutoriamente no apêndice C, discutindo a construção da sua álgebra nas várias dimensões de interesse na dissertação. Matrizes singulares são apresentadas rapidamente no apêndice $\mathrm{D}$, incluindo uma demonstração da escolha do calibre do cone de luz no formalismo de Green-Schwarz. A simetria conforme, relevante no estudo das teorias duais na correspondência $\mathrm{AdS} / \mathrm{CFT}$, é apresentada no último apêndice, E. 


\section{Capítulo 2}

\section{Corda Bosônica}

Neste capítulo é introduzida a teoria de cordas bosônicas e algumas de suas particularidades. A teoria quântica é obtida com a escolha do calibre do cone de luz e o espectro de estados da corda aberta e da corda fechada é analisado. Discute-se também a dualidade $\mathrm{T}$, inferindo a existência das D-branas e discutindo algumas de suas implicações. A base do desenvolvimento deste capítulo pode ser encontrada em $[3,4,5,6]$.

\subsection{A Ação da $p$-brana}

Ao estender o estudo da dinâmica de objetos pontuais (dimensão $p=0$ ) à objetos de dimensão $p \geq 1$ o procedimento mais natural é analisar possíveis generalizações da ação $S_{0}$ da partícula relativística.

A ação de uma partícula relativística é proporcional ao comprimento da sua linha-mundo, sua trajetória no espaço-tempo. Trajetórias de objetos de dimensão $p$ (p-branas) geram superfícies $p+1$ dimensionais ao se propagarem. Portando é natural construir sua ação $S_{p}$ na forma

$$
S_{p} \propto \int \sqrt{-\operatorname{det}\left(g^{\mu \nu} \partial^{\alpha} X_{\mu} \partial^{\beta} X_{\nu}\right)} d^{p+1} \sigma,
$$

na qual o integrando é um elemento de volume $p+1$ dimensional, $\sigma_{\alpha}(\operatorname{com} \alpha=0,1, \ldots, p)$ é a parametrização escolhida para descrever o volume-mundo e $X_{\mu}(\sigma)(\operatorname{com} \mu=0,1, \ldots, D-1)$ são as coordenadas da p-brana no espaço em que se move, chamado espaço-alvo (a dimensão do espaço-alvo é menor ou igual à dimensão $D$ do espaço-tempo).

Introduzindo um tensor métrico auxiliar $h_{\alpha \beta}$ no volume-mundo, é possível construir uma ação $S_{\sigma}$ classicamente equivalente à $S_{p}$, ou seja, que reproduz as mesmas equações de movimento:

$$
S_{\sigma}=\int \sqrt{-h}\left\{-\frac{T_{p}}{2} h^{\alpha \beta} \partial_{\alpha} X \cdot \partial_{\beta} X+\Lambda_{p}\right\} d^{p+1} \sigma .
$$

O termo cosmológico $\Lambda_{p}$ não é arbitrário. Isso é verificado da seguinte forma:

$$
\frac{\delta S_{\sigma}}{\delta h^{\alpha \beta}}=0 \Rightarrow T_{p}\left[\partial_{\alpha} X \cdot \partial_{\beta} X-\frac{1}{2} h_{\alpha \beta}\left(h^{\rho \gamma} \partial_{\rho} X \cdot \partial_{\gamma} X\right)\right]+\Lambda_{p} h_{\alpha \beta}=0 .
$$

Tomando o traço da equação acima:

$$
T_{p}\left(h^{\alpha \beta} \partial_{\alpha} X \cdot \partial_{\beta} X\right)=\frac{T_{p}}{2}(p+1)\left(h^{\alpha \beta} \partial_{\alpha} X \cdot \partial_{\beta} X\right)-\Lambda_{p}(p+1) .
$$


Como $\Lambda_{p}$ independe de $X^{\mu}$, a única solução possível é $\partial_{\alpha} X \cdot \partial_{\beta} X \propto h_{\alpha \beta}$. Substituindo esse resultado na equação acima, obtem-se $\Lambda_{p} \propto \frac{T_{p}}{2}(p-1)$.

\subsection{A Ação da corda bosônica}

A ação da corda bosônica (1-brana) é um caso particular das ações discutidas anteriormente. Ao se propagar, a corda forma uma superfície bidimensional no espaço-tempo, a folha-mundo, e são necessários dois parâmetros para descrevê-la: $\sigma^{0} \equiv \tau$ e $\sigma^{1} \equiv \sigma$. De forma geral, $\tau$ estará associado a um tempo na folha-mundo e $\sigma \in[0, \pi]$, tal que o intervalo definido cobre univocamente todos os pontos da corda para um dado $\tau$. Para $p=1, \Lambda_{p}$ anula-se em $S_{\sigma}$ e, além disso, uma possível dinâmica da métrica é limitada, uma vez que o termo de Einstein é um invariante topológico em duas dimensões, proporcional à característica de Euler da superfície descrita ${ }^{1}$. A ação da corda é, portanto ${ }^{2}$ :

$$
S_{b}=-\left(\frac{T_{1}}{2}\right) \int \sqrt{-h}\left\{h^{\alpha \beta} \partial_{\alpha} X \cdot \partial_{\beta} X\right\} d^{2} \sigma .
$$

Por uma simples análise dimensional, conclui-se que o parâmetro $T_{1}$ tem dimensão $\left[T_{1}\right]=M / T$. Em uma analogia ingênua com a ação da partícula relativística, $T_{1}=\lambda c$, em que $\lambda$ é uma densidade linear de massa e $c$ é a velocidade da luz no vácuo. Porém tal interpretação não é compatível por duas razões principais: como uma teoria fundamental da natureza, a teoria de cordas deve descrever, por exemplo, os bósons de calibre, que não possuem massa; e as soluções clássicas implicam que os extremos da corda devem mover-se com velocidade $c$. Como o objeto em questão é uma corda, $T_{1}=T / c$, em que $T$ é interpretado como sua tensão. No sistema natural de unidades $(\hbar=c=1), T$ está associado ao comprimento $l_{s}$ da corda por $T=\frac{1}{\pi l_{s}^{2}}$.

Como em qualquer outra teoria física, a análise das simetrias da ação (2.2) é bastante útil, permitindo fixar de modo conveniente a métrica auxiliar. $S_{b}$ apresenta as seguintes simetrias:

- Transformação de Poincaré

A simetria de Poincaré é evidente e pode ser resumida por $\delta X^{\mu}=a^{\mu}{ }_{\nu} X^{\nu}+b^{\mu}$. O parâmetro de translação é $b^{\mu}$, enquanto o parâmetro da transformação de Lorentz é $a^{\mu}{ }_{\nu}$;

- Reparametrização da Folha-Mundo (Difeomorfismo)

De forma geral:

$$
\sigma_{a} \rightarrow\left\{\begin{array}{l}
f^{\alpha}(\tau, \sigma)=\sigma^{\alpha} \\
h_{\alpha \beta}(\tau, \sigma)=\frac{\partial f^{\rho}}{\partial \sigma^{\alpha}} \frac{\partial f^{\gamma}}{\partial \sigma^{\beta}} h_{\rho \gamma}\left(\tau^{\prime}, \sigma^{\prime}\right)
\end{array}\right.
$$

- Transformação de Weyl

A simetria de Weyl corresponde, basicamente, a um reescalonamento da métrica na folha-mundo: $h^{\alpha \beta} \rightarrow \lambda(\tau, \sigma) h^{\alpha \beta}$.

Tomando uma variação da ação $S_{b}$ em relação a métrica auxiliar $h^{\alpha \beta}$, verifica-se que essa simetria implica que o traço do tensor energia-momento $\left(T^{\alpha \beta} h_{\alpha \beta}\right)$ é nulo.

Devido às duas últimas simetrias, $h^{\alpha \beta}$ pode ser fixada completamente (duas simetrias de reparametrização e uma simetria de Weyl fixando as três componentes independentes da métrica auxiliar).

\footnotetext{
${ }^{1}$ De fato, ao considerar um espaço-tempo genérico, o papel do termo de Einstein é importante pois dá o acoplamento com um dos campos de fundo. Mais detalhes são fornecidos na seção 2.6.2.

${ }^{2}$ Desse ponto em diante será utilizada a métrica plana $\eta_{\mu \nu}=(-1,1, \ldots, 1)$, a não ser que seja explicitamente dito o contrário.
} 


\subsection{Equações de Movimento e Soluções}

A ação $S_{b}$ fornece dois conjuntos de equações de movimento. Um associado à métrica auxiliar,

$$
\partial_{\alpha} X^{\mu} \partial_{\beta} X_{\mu}-\frac{1}{2} h_{\alpha \beta} h^{\gamma \rho} \partial_{\gamma} X^{\mu} \partial_{\rho} X_{\mu}=0
$$

e outro associado aos campos $X^{\mu}(\tau, \sigma)$,

$$
\partial_{\alpha}\left(\sqrt{-h} h^{\alpha \beta} \partial_{\beta} X^{\mu}\right)=0
$$

Para o caso de folhas-mundo descritas pelas cordas abertas e fechadas livres (em outras palavras, superfícies sem excentricidades topológicas), é possível escolher o calibre conforme, em que

$$
h_{\alpha \beta}=\eta_{\alpha \beta}=\left(\begin{array}{cc}
-1 & 0 \\
0 & 1
\end{array}\right)
$$

ou seja, a métrica plana. Definindo $P_{a}^{\mu} \equiv T \partial_{a} X^{\mu}$, a equação de movimento para $X^{\mu}$ é simplificada para

$$
\partial^{\alpha} P_{\alpha}^{\mu}=0
$$

e, uma vez que a métrica auxiliar foi fixada, sua equação de movimento deve ser imposta como vínculo:

$$
\left(P_{\tau} \pm P_{\sigma}\right)^{2}=0
$$

As equações de movimento podem ser reescritas nas coordenadas $\sigma_{ \pm}=\tau \pm \sigma$. Como $\partial_{ \pm}=\frac{1}{2}\left(\partial_{\tau} \pm \partial_{\sigma}\right)$, obtem-se:

$$
\partial_{+} \partial_{-} X^{\mu}=0 \quad\left(\partial_{+} X\right)^{2}=\left(\partial_{-} X\right)^{2}=0
$$

Pelas equações acima, $X^{\mu}(\tau, \sigma)$ pode ser decomposto em duas soluções que se propagam em direções opostas na folha-mundo, $X^{\mu}=X_{R}^{\mu}\left(\sigma_{-}\right)+X_{L}^{\mu}\left(\sigma_{+}\right)$. Expandindo em séries de Fourier complexas:

$$
\begin{aligned}
& X_{R}^{\mu}(\tau, \sigma)=\frac{x_{R}^{\mu}}{2}+a_{0}^{\mu}(\tau-\sigma)+\sum_{m \neq 0} \frac{1}{m} a_{m}^{\mu} e^{-i m(\tau-\sigma)} \\
& X_{L}^{\mu}(\tau, \sigma)=\frac{x_{L}^{\mu}}{2}+\tilde{a}_{0}^{\mu}(\tau+\sigma)+\sum_{m \neq 0} \frac{1}{m} \tilde{a}_{m}^{\mu} e^{-i m(\tau+\sigma)} .
\end{aligned}
$$

Embora não tenha sido mostrado explicitamente, o processo de derivação das equações de movimento envolve a fixação de termos de superfície. Portanto, as soluções de (2.5) devem ser fixadas pelas condições iniciais e pelas condições de contorno do problema (avaliadas nos extremos $\sigma^{*}=0, \pi$ ). A fixação das condições iniciais é bastante simples e não apresenta relevância na discussão subsequente. Já a fixação das condições de contorno, que são obtidas pelo termo de superfície

$$
\int d \tau\left[\left.\frac{\partial X^{\mu}}{\partial \sigma} \delta X_{\mu}\right|_{\sigma=\pi}-\left.\frac{\partial X^{\mu}}{\partial \sigma} \delta X_{\mu}\right|_{\sigma=0}\right]=0
$$

dá origem à duas famílias de soluções:

1. Cordas Fechadas

Tais soluções obedecem à condição de quasi-periodicidade $X^{\mu}(\tau, \sigma+\pi)=X^{\mu}(\tau, \sigma)+B^{\mu} \pi$. Pelas condições de contorno definidas, os modos de oscilação ímpares são nulos. Além disso $\tilde{a}_{0}^{\mu}-a_{0}^{\mu}=B^{\mu}$ 
e essa é a única relaçao entre os modos direitos $(\mathrm{R})$ e esquerdos $(\mathrm{L})^{3}$. De fato, a denominação corda fechada tem sentido apenas para $B^{\mu}=0$ (condição associada à simetria de translação em $\sigma$ e simetria de paridade $\sigma \leftrightarrow-\sigma$ na folha-mundo) ou no contexto de compactificação.

$$
\begin{aligned}
& X_{R}^{\mu}(\tau, \sigma)=\frac{x_{R}^{\mu}}{2}+l_{s} \alpha_{0}^{\mu}(\tau-\sigma)+i \frac{l_{s}}{2} \sum_{m \neq 0} \frac{1}{m} \alpha_{m}^{\mu} e^{-2 i m(\tau-\sigma)} \\
& X_{L}^{\mu}(\tau, \sigma)=\frac{x_{L}^{\mu}}{2}+l_{s} \tilde{\alpha}_{0}^{\mu}(\tau+\sigma)+i \frac{l_{s}}{2} \sum_{m \neq 0} \frac{1}{m} \tilde{\alpha}_{m}^{\mu} e^{-2 i m(\tau+\sigma)} .
\end{aligned}
$$

2. Cordas Abertas

(a) Condição de Extremo Fixo

A condição de Dirichlet, ou condição de extremo fixo, implica que $P_{\tau}^{\mu}\left(\tau, \sigma^{*}\right)=0$. Dessa forma, $a_{m}^{\mu}=-\tilde{a}_{m}^{\mu}$. Tal condição quebra a invariância de Poincaré (não há conservação de momento). Além disso é incompatível para $\mu=0$. Nesse caso $X^{0}$ seria estático, invalidando sua interpretação como coordenada temporal. Em geral:

$$
X^{\mu}(\tau, \sigma)=x^{\mu}+l_{s}^{2} w^{\mu} \sigma+i l_{s} \sum_{m \neq 0} \frac{1}{m} \alpha_{m}^{\mu} e^{-i m \tau} \sin (m \sigma) .
$$

(b) Condição de Extremo Livre

A condição de Neumann, ou condição de extremo livre, implica que $P_{\sigma}^{\mu}\left(\tau, \sigma^{*}\right)=0$ e, consequentemente, $a_{m}^{\mu}=\tilde{a}_{m}^{\mu}$. Através dos vínculos dados em (2.4), a condição de extremo livre implica que $P_{\tau}\left(\tau, \sigma^{*}\right)^{2}=0$. Ou seja, os extremos livres da corda bosônica descrita por $(2.2)$ movem-se à velocidade da luz. As soluções são da forma:

$$
X^{\mu}(\tau, \sigma)=x^{\mu}+l_{s}^{2} p^{\mu} \tau+i l_{s} \sum_{m \neq 0} \frac{1}{m} \alpha_{m}^{\mu} e^{-i m \tau} \cos (m \sigma) \text {. }
$$

(c) Condição Mista

As condições de contorno mistas possuem um extremo que satisfaz a condição $2 \mathrm{a}$ e um extremo que satisfaz a condição 2b. Por exemplo, ao considerar o extremo $\sigma=0$ fixo e o extremo $\sigma=\pi$ livre, obtem-se:

$$
X^{\mu}(\tau, \sigma)=x^{\mu}+\sum_{r \in \mathbb{Z}+\frac{1}{2}} \frac{1}{r} \alpha_{r}^{\mu} e^{-i r \tau} \sin (r \sigma) .
$$

Condições de contorno de Dirichlet e cordas fechadas com $B^{\mu} \neq 0$ serão discutidas apenas na seção 2.7. Condições mistas não serão abordadas.

A condição de realidade de $X^{\mu}$ implica que os modos de Fourier $a_{m}^{\mu}$ satisfazem à $\alpha_{-m}^{\mu}=\left(\alpha_{m}^{\mu}\right)^{\dagger}$. Além disso, os modos zero são identificados com o momento de translação da corda $p_{\mu}$. Para a corda fechada, $\alpha_{0}^{\mu}=\tilde{\alpha}_{0}^{\mu}=\frac{1}{2} l_{s} p^{\mu}$. Para a corda aberta, $\alpha_{0}^{\mu}=l_{s} p^{\mu}$.

De posse das soluções clássicas, é possível calcular todas as correntes conservadas na folha-mundo e, consequentemente, obter os observáveis de relevância, como a energia e o espectro de massa clássicos.

$$
\begin{aligned}
P_{\alpha}^{\mu}=T \partial_{\alpha} X^{\mu} & \rightarrow \text { Simetria de Translação. } \\
J_{\alpha}^{\mu \nu}=X^{\mu} P_{\alpha}^{\nu}-X^{\nu} P_{\alpha}^{\mu} & \rightarrow \text { Simetria de Lorentz. } \\
T_{\alpha \beta}=P_{\alpha} \cdot P_{\beta}-(\text { traço }) & \rightarrow \text { Tensor Energia-Momento. }
\end{aligned}
$$

\footnotetext{
${ }^{3}$ Há ainda um tipo de projeção que pode relacionar os modos da teoria que está associado à inversão dos extremos da corda, basicamente pela troca $\sigma \leftrightarrow \pi-\sigma$ nas soluções. Cordas cujas soluções clássicas são simétricas por essa transformação são denominadas não orientadas.
} 
Calculando as cargas conservadas, são obtidos os geradores de simetria do espaço-tempo e a hamiltoniana $H$ da corda bosônica. A simetria de translação é trivial. Os geradores de Lorentz são dados por:

- Corda Fechada

$$
J^{\mu \nu}=x^{\mu} p^{\nu}-x^{\nu} p^{\mu}-i \sum_{n=1}^{\infty} \frac{1}{n}\left(\alpha_{-n}^{\mu} \alpha_{n}^{\nu}-\alpha_{-n}^{\nu} \alpha_{n}^{\mu}+\tilde{\alpha}_{-n}^{\mu} \tilde{\alpha}_{n}^{\nu}-\tilde{\alpha}_{-n}^{\nu} \tilde{\alpha}_{n}^{\mu}\right) .
$$

- Corda Aberta

$$
J^{\mu \nu}=x^{\mu} p^{\nu}-x^{\nu} p^{\mu}-i \sum_{n=1}^{\infty} \frac{1}{n}\left(\alpha_{-n}^{\mu} \alpha_{n}^{\nu}-\alpha_{-n}^{\nu} \alpha_{n}^{\mu}\right)
$$

\subsection{Massa e Energia da Corda}

O tensor energia-momento $T_{\alpha \beta}$ é proporcional à variação da ação $S_{b}$ em relação à métrica auxiliar. De especial interesse são as componentes $T_{++}$e $T_{--}$, cujos modos de Fourier são dados por:

$$
L_{m}=\frac{1}{2} \sum_{n=-\infty}^{\infty} \alpha_{m-n} \cdot \alpha_{n} \quad \tilde{L}_{m}=\frac{1}{2} \sum_{n=-\infty}^{\infty} \tilde{\alpha}_{m-n} \cdot \tilde{\alpha}_{n}
$$

$L_{m}$ e $\tilde{L}_{m}$ são conhecidos como geradores de Virasoro e possuem estreita correspondência com os geradores da simetria conforme em duas dimensões ${ }^{4}$.

Uma vez que o tensor energia-momento deve ser nulo, cada um dos geradores de Virasoro deve se anular independentemente, ou seja, os vínculos da equação de movimento da métrica manisfestam-se como $L_{m}=0$ e $\tilde{L}_{m}=0$ na teoria clássica. Esses são os vínculos de Virasoro. Em particular, $L_{0}=\tilde{L}_{0}=0$ implica uma fórmula para a massa da corda, já que esta é calculada à partir dos modos zero da solução clássica, $M^{2}=-p^{\mu} p_{\mu}$.

- Corda Fechada

$$
\left.\begin{array}{l}
T_{++}=2 l_{s}^{2} \sum_{m=-\infty}^{\infty} \tilde{L}_{m} e^{-2 i m(\tau+\sigma)} \\
T_{--}=2 l_{s}^{2} \sum_{m=-\infty}^{\infty} L_{m} e^{-2 i m(\tau-\sigma)}
\end{array}\right\} \quad \begin{aligned}
& H=2\left(L_{0}+\tilde{L}_{0}\right) \\
& M^{2}=\frac{4}{l_{s}^{2}} \sum_{n=1}^{\infty}\left(\alpha_{-n} \cdot \alpha_{n}+\tilde{\alpha}_{-n} \cdot \tilde{\alpha}_{n}\right)
\end{aligned}
$$

- Corda Aberta

$$
\left.\begin{array}{l}
T_{++}=l_{s}^{2} \sum_{m=-\infty}^{\infty} L_{m} e^{-i m(\tau+\sigma)} \\
T_{--}=l_{s}^{2} \sum_{m=-\infty}^{\infty} L_{m} e^{-i m(\tau-\sigma)}
\end{array}\right\} \quad \begin{aligned}
& H=L_{0} \\
& M^{2}=\frac{2}{l_{s}^{2}} \sum_{n=1}^{\infty} \alpha_{-n} \cdot \alpha_{n}
\end{aligned}
$$

O espectro de massa da teoria clássica é contínuo, entretanto é evidente que não é positivo definido. A teoria quântica também está sujeita a tal irregularidade, embora sua manifestação seja através de estados de norma negativa. De fato, não há inconsistências. Apesar da determinação explícita das soluções clássicas, não há garantia alguma de que satisfaçam aos vínculos de Virasoro. Há ainda uma simetria escondida que permite a imposição dos vínculos de forma bastante simples, como será visto a seguir.

\footnotetext{
${ }^{4}$ Algumas propriedades do grupo conforme são discutidas no apêndice E.
} 


\subsection{O Calibre do Cone de Luz}

Ao considerar as simetrias da ação $S_{b}$ foram citadas a simetria de reparametrização na folha-mundo e o escalonamento de Weyl. Uma vez fixado o calibre conforme é possível ainda fazer uso de tais simetrias deixando a métrica auxiliar inalterada. Para transformações infinitesimais $\sigma_{a}^{\prime}=\sigma_{a}+\epsilon_{a}$ e $\eta_{a b}=\eta_{a b}-\Lambda \eta_{a b}$, basta que

$$
\partial_{a} \epsilon_{b}+\partial_{b} \epsilon_{a}=\Lambda \eta_{a b}+\mathcal{O}\left(\epsilon^{2}, \Lambda \epsilon\right)
$$

para que o calibre escolhido seja mantido. Ou seja, as transformações $\sigma_{ \pm} \rightarrow f_{ \pm}\left(\sigma_{ \pm}\right)$são uma simetria residual. Levando isso em conta e lembrando que $\partial_{+} \partial_{-} f_{ \pm}=0$, escolhe-se a parametrização de modo que $X^{+}(\tau, \sigma)=x^{+}+l_{s}^{2} p^{+} \tau$, em que

$$
X^{ \pm}=\left(X^{0} \pm X^{D-1}\right) / \sqrt{2}
$$

Essa escolha é denominada calibre do cone-de-luz. Agora, impondo os vínculos $\left(P_{\tau} \pm P_{\sigma}\right)^{2}=0$, obtem-se:

$$
\partial_{\tau} X^{-} \pm \partial_{\sigma} X^{-}=\frac{1}{2 p^{+} l_{s}^{2}}\left(\partial_{\tau} X^{i} \pm \partial_{\sigma} X^{i}\right)^{2}
$$

Substituindo as soluções, os modos $\alpha_{m}^{-}$são escritos em função dos modos ditos transversais, $\alpha_{m}^{i}$, com $i=1, \ldots, D-2$ :

$$
\alpha_{m}^{-}=\frac{1}{2 p^{+} l_{s}}\left(\sum_{n=-\infty}^{\infty} \alpha_{m-n}^{i} \alpha_{n}^{i}\right) .
$$

Dessa forma, os vínculos de Virasoro são satisfeitos automaticamente e o espectro de massa torna-se positivo definido, sendo constituído apenas pelos modos transversais. São definidos também os geradores de Virasoro transversais $L_{m}^{T}$ e $\tilde{L}_{m}^{T}$ :

$$
L_{m}^{T}=\frac{1}{2} \sum_{n=-\infty}^{\infty} \sum_{i=1}^{D-2} \alpha_{m-n}^{i} \alpha_{n}^{i} \quad \tilde{L}_{m}^{T}=\frac{1}{2} \sum_{n=-\infty}^{\infty} \sum_{i=1}^{D-2} \tilde{\alpha}_{m-n}^{i} \tilde{\alpha}_{n}^{i}
$$

Rumo à quantização da teoria, é importante analisar quais são os parênteses de Poisson associados aos modos normais $\alpha_{m}^{\mu}$.

Baseando-se nas relações fundamentais

$$
\begin{gathered}
\left\{P_{0}^{\mu}(\tau, \sigma), P_{0}^{\nu}\left(\tau, \sigma^{\prime}\right)\right\}_{P P}=\left\{X^{\mu}(\tau, \sigma), X^{\nu}\left(\tau, \sigma^{\prime}\right)\right\}_{P P}=0 \\
\left\{P_{0}^{\mu}(\tau, \sigma), X^{\nu}\left(\tau, \sigma^{\prime}\right)\right\}_{P P}=\eta^{\mu \nu} \delta\left(\sigma-\sigma^{\prime}\right)
\end{gathered}
$$

verifica-se que

$$
\begin{aligned}
\left\{p^{+}, x^{-}\right\}_{P P} & =-1 \\
\left\{p^{i}, x^{j}\right\}_{P P} & =\eta^{i j} \\
\left\{\alpha_{m}^{i}, \tilde{\alpha}_{n}^{j}\right\}_{P P} & =0 \\
\left\{\alpha_{m}^{i}, \alpha_{n}^{j}\right\}_{P P}=\left\{\tilde{\alpha}_{m}^{i}, \tilde{\alpha}_{n}^{j}\right\}_{P P} & =i m \delta_{m+n, 0} \eta^{i j},
\end{aligned}
$$

Como todos os vínculos foram eliminados, as relações acima são facilmente estendidas à teoria quântica no procedimento de quantização canônico. Esse é o caminho mais simples para o estudo da teoria quântica e seus vários aspectos. Um procedimento mais elegante, explicitamente covariante, é a quantização BRST. Entretanto, ao menos no nível de discussão proposto, tal alternativa não oferece vantagens e não será abordada.Adota-se em todo o texto o procedimento canônico. 


\subsection{Quantização}

O procedimento de quantização canônico se dá pela substituição dos parênteses de Poisson pelos comutadores $\left(\{,\}_{P P} \rightarrow i[],\right)$, promovendo os modos de expansão $\alpha_{m}^{\mu}$ à operadores. Desse modo, as equações (2.15) tornam-se:

$$
\begin{aligned}
{\left[x^{-}, p^{+}\right] } & =-i \\
{\left[x^{i}, p^{j}\right] } & =i \eta^{i j} \\
{\left[\alpha_{m}^{i}, \tilde{\alpha}_{n}^{j}\right] } & =0 \\
{\left[\alpha_{m}^{i}, \alpha_{n}^{j}\right]=\left[\tilde{\alpha}_{m}^{i}, \tilde{\alpha}_{n}^{j}\right] } & =m \delta_{m+n, 0} \eta^{i j} .
\end{aligned}
$$

A álgebra dos osciladores assemelha-se à uma álgebra de criação e aniquilação:

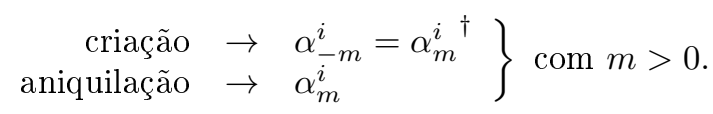

Junto aos operadores de criação e aniquilação é definido também o estado fundamental $\left|0, p^{+}, p^{i}\right\rangle$, em que $p^{+}$e $p^{i}$ designam as componentes do momento associado ao estado. Assim definido, os estados da corda são construídos pela atuação dos operadores de criação no estado fundamental. Para a corda aberta:

$$
\begin{aligned}
|\phi\rangle & =\alpha_{-m_{1}}^{i_{1}} \ldots \alpha_{-m_{k}}^{i_{k}}|0, p\rangle, \\
P^{\mu}|\phi\rangle & =p^{\mu}|\phi\rangle .
\end{aligned}
$$

Os estados da corda fechada são definidos de maneira análoga, sendo compostos por um produto direto de estados de modos esquerdos e direitos. É importante notar que estes são estados de apenas uma corda e não várias, pois esse é um procedimento de primeira quantização.

Uma vez introduzidas as relações de (2.16), é necessário avaliar como grandezas definidas classicamente, e.g. a energia de uma uma corda, são afetadas pela quantização. Em primeiro lugar define-se um ordenamento, afinal há ambiguidades na definição do produto de grandezas que não comutam. Por conveniência o ordenamento normal é escolhido, no qual os operadores de criação ficam à direita dos operadores de aniquilação. Produtos normalmente ordenados serão representados sempre entre um par ":. Por exemplo:

$$
: \alpha_{m}^{i} \alpha_{n}^{j}:=\left\{\begin{array}{l}
\alpha_{m}^{i} \alpha_{n}^{j}, \text { para } m<n \\
\alpha_{n}^{j} \alpha_{m}^{i}, \text { para } n<m
\end{array}\right.
$$

Dentre os objetos estudados, apenas os geradores $L_{0}, \tilde{L}_{0}$ e os geradores transversais associados são afetados pelo ordenamento. Para corrigir isso é introduzida uma constante de ordenamento " $a$ ", tal que:

$$
L_{0_{\text {clássico }}}=\frac{1}{2} \sum_{m=-\infty}^{\infty} \alpha_{-m} \cdot \alpha_{m} \rightarrow \frac{1}{2} \sum_{m=-\infty}^{\infty}: \alpha_{-m} \cdot \alpha_{m}:-a=L_{0_{\text {quântico }}}-a .
$$

Como os geradores de Virasoro de modo zero estão associados à hamiltoniana $H$ do sistema, essa nova contribuição é entendida como a energia de ponto zero na teoria quântica.

Tal constante reflete sua importância na definição do operador de massa $M^{2}$ :

- Corda Fechada

$$
M^{2}=\frac{4}{l_{s}^{2}}(N+\tilde{N}-a-\tilde{a})
$$


- Corda Aberta

$$
M^{2}=\frac{2}{l_{s}^{2}}(N-a)
$$

$N$ e $\tilde{N}$ são operadores de contagem (ou operadores número) e suas expressões são:

$$
N=\sum_{m=1}^{\infty} \alpha_{-m}^{i} \cdot \alpha_{m}^{i} \quad \tilde{N}=\sum_{m=1}^{\infty} \tilde{\alpha}_{-m}^{i} \cdot \tilde{\alpha}_{m}^{i}
$$

Devido ao vínculo entre os modos zero da corda fechada $\left(\alpha_{0}^{\mu}=\tilde{\alpha}_{0}^{\mu}\right)$, obtem-se que $a=\tilde{a}$ e $N=\tilde{N}$. A última igualdade é conhecida como condição de nivelamento das cordas fechadas. Outro modo de obter essa condição é fixar a simetria de translação em $\sigma$, impondo que o gerador de tal simetria aniquile os estados $|\phi\rangle$.

Tendo partido de um teoria explicitamente covariante e fixando os calibres de simetria interna espera-se que o resultado final mantenha a simetria de Lorentz. Ao escolher o calibre do cone de luz são definidas direções privilegiadas no espaço-tempo e é possível que a quebra da simetria de Lorentz manifeste-se através de anomalias na teoria quântica. Requerendo tal simetria, mesmo no nível quântico, a teoria de cordas fornece um de seus resultados mais polêmicos e mais especiais, ao menos no sentido de teoria fundamental da natureza, indicando o número de dimensões que o espaço-tempo deve possuir.

A análise do espectro físico da teoria mostra que o primeiro estado excitado pertence à uma representação do grupo $S O(D-2)$, correspondendo, basicamente, às direções transversais. Para a corda aberta, por exemplo, tais estados são dados por $\alpha_{-1}^{i}|0, p\rangle$ e sua massa é $M^{2}=l_{s}^{2}(1-a) / 2$. Na teoria de representações, os estados não massivos pertencentes ao grupo de Lorentz devem pertencer à uma representação do subgrupo $S O(D-2)$. Ou seja, para uma teoria consistente $a=1$. Continuando, a origem da constante " $a$ " é o ordenamento de $L_{0}^{T}$ e corresponde à

$$
a=\frac{1}{2} \sum_{m=1}^{\infty}\left[\alpha_{-m}^{i}, \alpha_{m}^{i}\right]=-(D-2) \sum_{m=1}^{\infty} m=\frac{D-2}{24} .
$$

Na equação acima foi utilizada a regularização da função $\zeta$ de Riemann. Fixando $a=1$ obtem-se $D=26$. Por uma simples análise verifica-se que a dimensão do espaço-tempo não pode ser qualquer para que a teoria quântica preserve a covariância clássica. De modo mais rigoroso, a imposição da algebra de Lorentz no nível quântico fornece os mesmos resultados e o cálculo é mostrado no apêndice A.

\subsubsection{Espectro da Corda Aberta}

O espectro físico de estados da corda aberta é obtido, como foi visto, através da ação dos operadores de criação $\alpha_{-m}^{i}$, com $m<0$, no estado fundamental $\left|0, p^{+}, p^{i}\right\rangle$ e sua massa é dada por $M^{2}=\frac{2}{l_{s}^{2}}(N-1)$.

- $N=0$ corresponde ao estado fundamental $\left|0, p^{+}, p^{i}\right\rangle$ e possui massa imaginária. Esse estado é denominado táquion. Sua presença é indesejada e geralmente está associado à instabilidades do vácuo da teoria;

- $N=1$ corresponde aos estados $\alpha_{-1}^{i}\left|0, p^{+}, p^{i}\right\rangle$ e possuem massa nula. Esses estados não-massivos possuem $D-2=24$ polarizações transversais, propriedade comum aos campos de calibre. Usualmente é identificado com o fóton em 26 dimensões;

- $N=2$ engloba os estados $\alpha_{-1}^{i} \alpha_{-1}^{j}\left|0, p^{+}, p^{i}\right\rangle$ e $\alpha_{-2}^{i}\left|0, p^{+}, p^{i}\right\rangle$. São os primeiros estados massivos do espectro da corda aberta. Possuem, ao todo, 324 graus de liberdade e pertencem à representação dos tensores simétricos de segunda ordem de $S O(D-1)$; 
- $N>2$ correspondem à estados massivos associados à representações tensoriais de $S O(D-1)$. Embora seja bastante interessante o fato da teoria possuir um espectro massivo infinito, sua descrição não é relevante para a dissertação.

\subsubsection{Espectro da Corda Fechada}

O espectro físico dos estados da corda fechada é obtido à partir da atuação dos modos de criação direitos e esquerdos, vinculados à condição de nivelamento $N=\tilde{N}$ e sua massa é dada por $M^{2}=$ $\frac{8}{l_{s}^{2}}(N-1)$.

- $N=0$ corresponde ao estado fundamental $\left|0, p^{+}, p^{i}\right\rangle$ e, como na corda aberta, é identificado com o táquion;

- $N=1$ corresponde aos estados $\alpha_{-1}^{i} \tilde{\alpha}_{-1}^{j}\left|0, p^{+}, p^{i}\right\rangle$ e possuem massa nula. Esses estados não massivos pertencem à representação dos tensores de segunda ordem do grupo $S O(D-2)$. São identificados com três campos fundamentais: a parte simétrica de traço nulo corresponde ao gráviton, $g_{\mu \nu}$; a parte antissimétrica corresponde ao campo de Kalb-Ramond, $B_{\mu \nu}$; e o escalar, formado pelo seu traço, corresponde ao dílaton, $\phi$;

- $N>1$ correspondem à estados massivos associados à representações tensoriais de $S O(D-1)$ e também não serão abordados neste texto.

Uma vez identificados os estados não massivos da corda fechada, são necessários alguns comentários sobre o seu papel na teoria. Até agora o espaço considerado para a obtenção do espectro físico foi o espaçoplano. É natural, portanto, acoplar tais estados como campos de fundo à ação (2.2): $S_{b}^{\prime}=S_{g}+S_{B}+S_{\phi}$.

$$
\begin{gathered}
S_{g}=-\left(\frac{1}{\pi l_{s}^{2}}\right) \int \sqrt{-h} h^{\alpha \beta} g_{\mu \nu}(X) \partial_{\alpha} X^{\mu} \cdot \partial_{\beta} X^{\nu} d^{2} \sigma . \\
S_{B}=-\left(\frac{1}{\pi l_{s}^{2}}\right) \int \varepsilon^{\alpha \beta} B_{\mu \nu}(X) \partial_{\alpha} X^{\mu} \cdot \partial_{\beta} X^{\nu} d^{2} \sigma . \\
S_{\phi}=-\left(\frac{1}{2 \pi}\right) \int \sqrt{-h} \phi(X) R^{(2)}(h) d^{2} \sigma .
\end{gathered}
$$

Nas expressões acima $\varepsilon^{\alpha \beta}$ é o símbolo de Levi-Civita em duas dimensões $\left(\varepsilon^{01}=1\right)$ e $R^{(2)}(h)$ é o escalar de curvatura associado à métrica $h^{\alpha \beta}$.

Para a obtenção do espectro gerado por $S_{b}^{\prime}$ é necessário conhecer o valor de cada um dos campos de fundo. Nesse sentido, a teoria é dita background dependente e o tratamento da geometria do espaçotempo, principalmente no nível quântico, é feito de forma perturbativa.

O campo de Kalb-Ramond é o análogo do potencial vetor $A_{\mu}$ eletromagnético para uma partícula e existe apenas no espectro das cordas orientadas.

O campo do dílaton atua como uma constante de acoplamento $g_{s}$ na teoria em interação. Mais precisamente, seu valor esperado no vácuo (VEV), $\phi_{0}$, está associado à constante de acoplamento como $g_{s}=e^{-\phi_{0}}$. A maneira mais simples de visualizar a relação é analisar o gerador funcional construído à partir de $S_{b}^{\prime}$ na versão euclidiana:

$$
Z \sim \int \mathcal{D} h \int \mathcal{D} X \ldots \exp \left\{-S_{b}^{\prime}\right\}
$$


O termo $\int \sqrt{-h} R^{(2)}(h) d^{2} \sigma$ é um invariante topológico proporcional à característica de Euler $\chi(\mathcal{M})$ da superfície (folha-mundo), tal que $\chi(\mathcal{M})=2-2 n_{h}-n_{b}-n_{c}$. Nessa expressão $n_{h}$ representa o genus da superfície, $n_{b}$ o número de bordas, $n_{c}$ o número de cross-caps e $\mathcal{M}$ a variedade em questão. Para superfícies fechadas orientadas, $\chi=2-2 n_{h}$,

$$
Z \sim \sum_{n_{h}=0}^{\infty} e^{\left(2-2 n_{h}\right) \phi_{0}} \int \mathcal{D} X \ldots \exp \left\{-S_{b}^{\prime}\right\} .
$$

Assim, ao considerar a ação $S_{\phi}$ no gerador funcional, a interpretação do VEV do dílaton como constante de acoplamento é imediata.

\section{7 $\quad$ Dualidade T e D-branas}

Como foi visto na seção 2.3, ao discutir a implementação das condições de contorno, as soluções clássicas da teoria de cordas bosônicas são compostas por campos que se propagam em direções opostas na folha-mundo, em geral

$$
X^{\mu}(\tau, \sigma)=X_{R}^{\mu}\left(\sigma_{-}\right)+X_{L}^{\mu}\left(\sigma_{+}\right) .
$$

Nota-se que uma transformação $X_{R}^{\mu} \rightarrow-X_{R}^{\mu}$ tem implicações muito interessantes. Nas soluções (2.8) e (2.9), há uma troca entre o termo de quasi-periodicidade $B^{\mu}$ e o momento linear. Nas soluções de cordas abertas, condições de extremos livres são levadas à condições de extremos fixos e vici-versa. Para visualizar de maneira mais consistente tal transformação é bastante útil estudá-la no contexto de compactificação, em particular a compactificação no toro. A compactificação toroidal é implementada identificando, à partir das direções transversais, as componentes das dimensões ordinárias $X^{i}$, com $i=1, \ldots, k$, e as componentes das dimensões compactas $Y^{I}$, com $I=k+1, \ldots, D-2$. Para as últimas, o momento linear é quantizado:

$$
p^{I}=\frac{1}{2}\left(p_{R}^{I}+p_{L}^{I}\right)=\frac{K^{I}}{R_{(I)}} .
$$

Aqui $K^{I} \in \mathbb{Z}$ é denominado modo de Kaluza-Klein e $R_{(I)}$ é o raio da dimensão compacta.

A existência de dimensões compactas implica uma mudança no espectro de massa das teorias estudadas, como será visto em seguida.

\subsubsection{Compactificação para Cordas Fechadas}

As condições de contorno da corda fechada são generalizadas para:

$$
\left\{\begin{array}{l}
X^{i}(\tau, \sigma+\pi)=X^{i}(\tau, \sigma) \\
Y^{I}(\tau, \sigma+\pi)=Y^{I}(\tau, \sigma)+2 \pi R_{(I)} W^{I}
\end{array}\right.
$$

Em que $W^{I} \in \mathbb{Z}$ é denominado número de circulação, representando o número de voltas que a corda dá ao redor da dimensão compacta. As soluções foram apresentadas em (2.8) e (2.9) e as condições de contorno acima implicam que:

$$
\left\{\begin{array}{l}
p^{i}=\frac{2}{l_{s}} \alpha_{0}^{i}=\frac{2}{l_{s}} \tilde{\alpha}_{0}^{i} \\
p^{I}=\frac{1}{l_{s}}\left(\alpha_{0}^{I}+\tilde{\alpha}_{0}^{I}\right)=\frac{K^{I}}{R_{(I)}} \\
W^{I}=\frac{l_{s}}{2 R_{(I)}}\left(\tilde{\alpha}_{0}^{I}-\alpha_{0}^{I}\right)
\end{array}\right.
$$


O estado fundamental na teoria quântica deve trazer as informações associadas às dimensões compactas, sendo designado por $\left|0, K^{I}, W^{I}, p^{+}, p^{i}\right\rangle$. Os estados excitados são construídos da maneira usual, sujeitos à condição de nivelamento

$$
N-\tilde{N}=\sum_{I=k+1}^{D-2} K^{I} W^{I}
$$

A expressão acima reflete o fato de que a simetria de translação em $\sigma$ é destruída. lação:

$\mathrm{O}$ operador de massa ${ }^{5}$ passa a incluir explicitamente os modos de Kaluza-Klein e números de circu-

$$
M^{2}=\sum_{I=k+1}^{D-2}\left[\left(\frac{K^{I}}{R_{(I)}}\right)^{2}+\left(\frac{2 W^{I} R_{(I)}}{l_{s}^{2}}\right)^{2}\right]+\frac{4}{l_{s}^{2}}(N+\tilde{N}) .
$$

Como esperado, quando o número de circulação é nulo e $R \rightarrow \infty$ recupera-se o resultado de cordas fechadas no espaço plano.

É notável a simetria $R \leftrightarrow l_{s}^{2}(2 \tilde{R})^{-1}$ e $K \leftrightarrow W$ na expressão de massa. Tal simetria é conhecida como dualidade $T$ e tem implicações fundamentais na interpretação da teoria, uma vez que estabelece um limite prático para o tamanho das dimensões compactas, a saber $R=\frac{l_{s}}{\sqrt{2}}$. Fazendo uma regressão às soluções clássicas, verifica-se que a simetria ${ }^{6}$ é mapeada precisamente pela transformação $X_{R}^{\mu} \rightarrow-X_{R}^{\mu}$, possibilitando a extensão da dualidade às cordas abertas.

\subsubsection{Compactificação para Cordas Abertas}

Quando são consideradas as condições de contorno de extremos livres, as soluções (2.11) continuam válidas, sendo que as componentes do momento nas direções compactas são quantizadas de acordo com (2.24). O espectro de massa passa a incluir explicitamente os modos de Kaluza-Klein:

$$
M^{2}=\sum_{I=k+1}^{D-2}\left(\frac{K^{I}}{R_{(I)}}\right)^{2}+\frac{2}{l_{s}^{2}} N .
$$

Ao aplicar a dualidade $\mathrm{T}$ à corda de extremos livres, verifica-se que a teoria resultante é equivalente àquela da corda com extremos fixos nas dimensões compactificadas. É possível inclusive definir um número de circulação $W^{I}$, de tal modo que o espectro de massa das duas teorias seja invariante pelas transformações $R \leftrightarrow l_{s}^{2}(2 \tilde{R})^{-1}$ e $K \leftrightarrow W$. Como $W^{I}$ é um número inteiro, os extremos são fixos no mesmo ponto do espaço, embora a corda esteja enrolada $\left|W^{I}\right|$ vezes sobre a dimensão compacta.

Nesse ponto fica claro que as cordas de extremos fixos não podem ser ignoradas, uma vez que constituem uma teoria dual àquela de cordas de extremos livres. A questão chave é: onde os extremos de uma corda são fixos? Como já foi comentado, esse tipo de solução não possui invariância translacional indicando que o momento não é conservado. Objetos chamados D-branas ("D" de Dirichlet) são introduzidos para corrigir essa deficiência.

Se uma corda possui condições de Dirichlet em $(D-1-p)$ dimensões, então ela está fixada em uma $\mathrm{D} p$-Brana e os extremos sobre a brana são livres. Estendendo o raciocínio, a teoria de cordas com extremos livres possui uma D25-brana, que preenche todo o espaço.

\footnotetext{
${ }^{5}$ É importante lembrar que a massa inercial de um objeto é definida à partir das componentes de momento linear nas dimensões ordinárias (não compactas).

${ }^{6}$ É possível estender a construção para uma teoria com campos de fundo constantes e o grupo de simetria da dualidade é $O(n, n, \mathbb{Z})$, sendo que $n$ representa o número de dimensões compactificadas.
} 
Consequentemente, a dualidade $\mathrm{T}$ também deve atuar nas D-Branas. Considerando uma $\mathrm{D} p$-brana, há duas possibilidades:

- se a dimensão sobre a qual a dualidade é aplicada é trasnversal à brana, equivalente a aplicar a dualidade a uma das componentes de extremos fixos da corda, a $\mathrm{D} p$-brana transforma-se em uma $\mathrm{D} p+1$-brana;

- do contrário, se a dimensão T-dual está sobre a brana, a D $p$-brana transforma-se em uma $\mathrm{D} p$ - 1brana.

As D-branas introduzem várias novas possibilidades no desenvolvimento da teoria e sua dinâmica será discutida no capítulo 5. A próxima seção discute a introdução da simetria de calibre, um ponto muito importante, já que o modelo padrão de partículas elementares inclui o grupo de simetria $S U(3)_{C} \otimes$ $S U(2)_{L} \otimes U(1)_{Y}$.

\subsection{Simetria de Calibre $U(N)$}

A simetria de calibre é inserida na teoria através das cargas de Chan-Paton. A idéia consiste em associar a cada extremo da corda uma representação fundamental de $U(n)$.

Para cordas orientadas associa-se uma representação fundamental para um extremo e anti-fundamental para outro. Assim, as cargas de Chan-Paton geram uma degenerescência de $n^{2}$ no espectro de massa. Os estados da corda ganham dois novos números quânticos, associados aos dois índices da representação de cada extremo, e são dados por $\left|N, p^{+}, p^{i}, a, b\right\rangle$. Naturalmente, tal estado transforma-se sob a representação adjunta de $U(n)$ :

$$
\left|N, p^{+}, p^{i}, a, b\right\rangle \rightarrow U_{a c} U_{b d}^{\dagger}\left|N, p^{+}, p^{i}, c, d\right\rangle .
$$

Na expressão acima, os índices latinos estão associados ao grupo $U(n)$, tal que $a=1,2, \ldots, n$.

Na presença de um campo de calibre plano $A$ (ou seja, campos cujo tensor $d A$ é nulo), há efeitos análogos ao efeito conhecido como Aharanov-Bohm. Considerando uma dimensão compactificada em um círculo de raio $R$, a linha de Wilson $\Upsilon$ nesta dimensão é dada por

$$
\Upsilon=\exp \left\{i \int_{0}^{2 \pi R} A d x\right\}
$$

que, após a diagonalização, fornece:

$$
\Upsilon=\operatorname{diag}\left(e^{i \theta_{1}}, \ldots, e^{i \theta_{n}}\right) .
$$

Portanto, na presença de uma linha de Wilson, o momento linear da corda na direção compactificada pode assumir valores fracionários. Assim,

$$
p=\frac{K}{R}-\frac{\theta_{a}-\theta_{b}}{2 \pi R}
$$

é a componente do momento na direção compactificada associada ao estado $\left|N, p^{+}, p^{i}, a, b\right\rangle$. Consequentemente, o espectro de massa para esse tipo de estado é alterado:

$$
M_{a b}^{2}=\left(\frac{K}{R}-\frac{\theta_{a}-\theta_{b}}{2 \pi R}\right)^{2}+\frac{2}{l_{s}^{2}} N .
$$


O correspondente em uma teoria T-dual seria um número de circulação não inteiro, mostrando que os extremos da corda estão presos a D-branas em diferentes pontos na dimensão compactificada.

É fácil notar também que a presença de uma linha de Wilson quebra a simetria de calibre $U(n)$ para $U(1)^{n}, \operatorname{com} \theta_{a} \neq \theta_{b}$. Para os casos em há componentes coincidentes, a simetria de calibre pertence a um grupo maior. Por exemplo, quando existem ao menos $m$ componentes coincidentes, há uma simetria de calibre $U(m) \otimes U(1)^{n-m}$.

Desse modo, uma possível interpretação para uma simetria de calibre $U(m)$ seria através de $m$ Dbranas conincidentes na dimensão compactificada.

Para cordas não-orientadas, ambos os extremos recebem uma representação fundamental, uma vez que estão identificados. Verifica-se, então, que o grupo de simetria é $S O(m)$ para extremos antissimétricos e $U S p(m)$ para extremos simétricos.

\subsection{Comentários}

A teoria bosônica não descreve, por razões óbvias, a natureza. Em primeiro lugar, contém o táquion. Por muito tempo acreditou-se que sua existência tornava a teoria inconsistente. Entretanto, com o conceito de D-branas, o táquion passou a ser interpretado como uma instabilidade da D25-brana e isso levou a várias discussões (e conclusões) que não serão descritas aqui. Outra "falha" evidente da teoria bosônica é o fato de não conter férmions em seu espectro. Com o objetivo de descrever tanto bósons como férmions foi introduzida a superssimetria, tornando a teoria mais robusta. Nos próximos capítulos são descritos dois formalismos que incorporam a supersimetria nas chamadas teorias de supercordas: o formalismo RNS, que trata campos fermiônicos na folha-mundo; e o formalismo de Green-Schwarz, pelo qual são introduzidos férmions no espaço-tempo. 


\section{Capítulo 3}

\section{Supercorda RNS}

Neste capítulo, a ação da corda bosônica é generalizada de modo a incluir a supersimetria local na folha-mundo ${ }^{1}$. A quantização da teoria é feita covariantemente pelo procedimento canônico, com uma discussão subsequente da álgebra de super Virasoro. Para a obtenção do espectro físico é escolhido o calibre do cone de luz, introduzindo em seguida a projeção GSO. A partir de uma análise do espectro da teoria, a supersimetria no espaço-tempo é sugerida pela demonstração da igualdade em número de graus de liberdade entre os estados bosônicos e fermiônicos. O desenvolvimento aqui apresentado reproduz em grande parte o respectivo conteúdo das referências [3, 4]. Algumas propriedades e resultados envolvendo matrizes de Dirac e supersimetria podem ser úteis de antemão e estão descritas nos apêndices B e C, respectivamente.

\subsection{Supersimetria Local e a Ação RNS}

Para construir a teoria de cordas supersimétrica, ou teoria de supercordas, é necessário, acreditando no potencial da corda bosônica, generalizar a ação (2.2). Tendo em vista o poder das simetrias da folha-mundo no desenvolvimento da teoria quântica, espera-se que tal ação possua todas as simetrias já discutidas no capítulo anterior. Com a inclusão dos campos fermiônicos, é natural que, além da supersimetria, algumas simetrias de mesmo caráter se manisfestem, o que realmente acontece. O resultado é o formalismo RNS (Ramond-Neveu-Schwarz) das supercordas.

As coordenadas do espaço-alvo da corda $X^{\mu}$ são escalares na folha-mundo, logo é necessário um espinor para que a álgebra de supersimetria seja fechada. Esse novo campo é descrito por uma variável de Grassman $\psi^{\mu}$ tal que $\left\{\psi^{\mu}, \psi^{\nu}\right\}=0$, sendo um espinor apenas na folha-mundo, carregando um índice vetorial do espaço-tempo.

O estudo de espinores em um espaço-tempo arbitrário (nesse caso, a folha-mundo) é feito através do formalismo das vielbein (zweibein, em 2 dimensões), designadas por $e_{\alpha}^{a}$. O índice latino corresponde a um índice de Lorentz local e o índice grego corresponde ao índice geral de coordenadas. Nesse formalismo o campo fundamental deixa de ser a métrica $h_{\alpha \beta}$ e passa a ser a zweibein, construída de tal forma que $h_{\alpha \beta}=e_{\alpha}^{a} e_{\beta}^{b} \eta_{a b}$, sendo $\eta_{a b}$ a métrica plana. Além disso, são definidas também as inversas: $e_{\alpha}^{a} e_{a}^{\beta}=\delta_{\alpha}^{\beta}$; e $e_{\alpha}^{a} e_{b}^{\alpha}=\delta_{b}^{a}$. Como a métrica possui 3 graus de liberdade, parece haver um excesso na introdução de $e_{\alpha}^{a}$, uma vez que possui 4 componentes. Entretanto, há ainda a simetria de Lorentz local, o que torna os graus de liberdade da parte antissimétrica do produto $e_{\alpha}^{a} e_{\beta}^{b}$ não físicos.

\footnotetext{
${ }^{1}$ Neste capítulo o termo local refere-se exclusivamente à folha-mundo e não ao espaço-tempo.
} 
Analogamente a uma teoria de calibre, a simetria de Lorentz local pode ser associada a um potencial, nesse caso à conexão de spin, $\omega_{\alpha}=\omega_{\alpha}^{a b} \Gamma_{a b}$, em que $\Gamma_{a b}$ é o produto antissimétrico das matrizes de Dirac $\Gamma_{a}$ em duas dimensões. Sob uma transformação de Lorentz $L(x)$, a conexão de spin deve transformar-se como:

$$
\omega_{\alpha} \rightarrow L \omega_{\alpha} L^{-1}-\left(\partial_{\alpha} L\right) L^{-1}
$$

Sob a mesma transformação, $\psi \rightarrow L \psi$. Assim, a derivada covariante de $\psi$,

$$
D_{\alpha} \psi=\left(\partial_{\alpha}+\omega_{\alpha}\right) \psi
$$

transforma-se também como um espinor na folha-mundo.

Definindo $\Gamma^{\alpha}(\tau, \sigma) \equiv \Gamma^{a} e_{a}^{\alpha}$, a ação de um espinor é escrita como

$$
S_{\psi}=i \int d^{2} \sigma \sqrt{-h} \bar{\psi} \Gamma^{\alpha} D_{\alpha} \psi
$$

A derivada covariante pode, neste caso, ser substituída pela derivada ordinária. A razão é simples. O termo da conexão de spin fornece um bilinear $\bar{\psi} \Gamma^{a} \Gamma^{b c} \psi$ o que, em duas dimensões é equivalente a $\bar{\psi} \Gamma^{d} \psi$, uma vez que existem apenas duas matrizes de Dirac. Pela propriedade (B.4) do apêndice, tal contribuição é nula.

Assim, além das coordenadas do espaço-alvo, a ação da corda supersimétrica deve incorporar novas coordenadas fermiônicas, de tal forma que:

$$
S^{\prime}=-\left(\frac{T}{2}\right) \int \sqrt{-h}\left\{h^{\alpha \beta} \partial_{\alpha} X \cdot \partial_{\beta} X+i \bar{\psi} \cdot \Gamma^{\alpha} \partial_{\alpha} \psi\right\} d^{2} \sigma
$$

Ao considerar a métrica plana $h_{\alpha \beta}=\eta_{\alpha \beta}$ e $e_{a}^{\alpha}=\delta_{a}^{\alpha}$, a ação acima é invariante, a menos de termos de superfície, pelas transformações $\delta X^{\mu}=\bar{\xi} \psi^{\mu}$ e $\delta \psi^{\mu}=-i\left(\Gamma^{\alpha} \xi\right) \partial_{\alpha} X^{\mu}$, sendo $\xi$ o parâmetro global das transformações de supersimetria. Nota-se que a condição de realidade de $X^{\mu}$ foi estendida aos espinores $\psi^{\mu}$, de modo que serão considerados espinores de Majorana. Essa condição é importante pois em qualquer teoria supersimétrica o número de graus de liberdade bosônicos e fermiônicos é igual ${ }^{2}$.

Quando a supersimetria local é requerida, é natural considerar transformações da métrica da folhamundo. Ao fazer isso, é necessário introduzir um campo fermiônico de spin mais alto que $\psi^{\mu}$, o espinor de Rarita-Schwinger $\chi_{\alpha}$. Basicamente, um espinor com um índice vetorial da folha-mundo. Sua transformação de supersimetria deve deixar claro seu caráter local ( $\chi_{\alpha}$ é o campo de calibre associado à supersimetria local) e o procedimento de Noether aplicado à ação $S^{\prime}$ proporciona exatamente isso. Verifica-se que a partir da transformação $\delta \chi_{\alpha}=D_{\alpha} \xi$ são gerados recursivamente os termos necessários para a ação supersimétrica. É possível mostrar que a ação mais geral com supersimetria local que contém $S^{\prime}$ é dada por:

$$
\begin{aligned}
S^{\prime \prime} & =-\left(\frac{T}{2}\right) \int \sqrt{-h}\left\{h^{\alpha \beta} \partial_{\alpha} X \cdot \partial_{\beta} X+i \bar{\psi} \cdot \Gamma^{\alpha} \partial_{\alpha} \psi+2 \bar{\chi}_{\alpha} \Gamma^{\beta} \Gamma^{\alpha} \psi \cdot \partial_{\beta} X+\frac{1}{2} \bar{\psi} \cdot \psi \bar{\chi}_{\alpha} \Gamma^{\beta} \Gamma^{\alpha} \chi_{\beta}\right\} d^{2} \sigma \\
& -\int \sqrt{-h}\left\{\frac{1}{2 \kappa^{2}} R+\frac{i}{2} \bar{\chi}_{\alpha} \Gamma^{\alpha \beta \lambda} \partial_{\beta} \chi_{\lambda}\right\} d^{2} \sigma
\end{aligned}
$$

A última integral na ação acima contém os termos cinéticos de $e_{\alpha}^{a}$ e de $\chi_{\alpha}$, sendo que $\kappa$ designa a constante de acoplamento gravitacional bidimensional, e sua contribuição não apresenta relevância. Em primeiro lugar, a zweibein não possui dinâmica e seu termo cinético corresponde a um invariante topológico, como

\footnotetext{
${ }^{2}$ Nesse caso, atinge-se a igualdade apenas na concha de massa, i.e. utilizando as equações de movimento. Para conseguir uma ação manisfestamente supersimétrica são incluídos graus de liberdade bosônicos não propagantes.
} 
já foi visto no capítulo 2. Além disso, não é possível construir um produto antissimétrico de três matrizes de Dirac, uma vez que há apenas duas para tal, de modo que $\Gamma^{\alpha \beta \lambda}=0$.

Com essa análise, é obtida a ação do formalismo RNS da teoria de supercordas, na qual a supersimetria manifesta-se na folha-mundo:

$$
\begin{aligned}
S_{R N S}= & -\left(\frac{T}{2}\right) \int \sqrt{-h}\left\{h^{\alpha \beta} \partial_{\alpha} X \cdot \partial_{\beta} X+i \bar{\psi} \cdot \Gamma^{\alpha} \partial_{\alpha} \psi\right. \\
& \left.+2 \bar{\chi}_{\alpha} \Gamma^{\beta} \Gamma^{\alpha} \psi \cdot \partial_{\beta} X+\frac{1}{2} \bar{\psi} \cdot \psi \bar{\chi}_{\alpha} \Gamma^{\beta} \Gamma^{\alpha} \chi_{\beta}\right\} d^{2} \sigma .
\end{aligned}
$$

A ação (3.1) possui as seguintes simetrias:

- Reparametrização da Folha-Mundo

- Simetria de Weyl Local

$$
\begin{array}{cc}
\delta X^{\mu}=0 & \delta \psi^{\mu}=-\frac{1}{2} \Lambda \psi^{\mu} \\
\delta e_{\alpha}^{a}=\Lambda e_{\alpha}^{a} & \delta \chi_{\alpha}=\frac{1}{2} \Lambda \chi_{\alpha}
\end{array}
$$

- Simetria Fermiônica Local (SFL)

$$
\delta X^{\mu}=0 \quad \begin{gathered}
\delta \chi_{\alpha}=i\left(\Gamma_{\alpha} \chi\right) \\
\delta \psi^{\mu}=0 \quad \delta e_{\alpha}^{a}=0
\end{gathered}
$$

Aqui $\chi$ é um espinor de Majorana. Tal simetria é exclusiva do campo de Rarita-Schwinger, sendo característica do modelo bidimensional. A verificação é imediata a partir da identidade (B.3) do apêndice;

- Simetria de Lorentz Local

- Simetria de Poincaré

A única diferença para a corda bosônica é a presença do vetor $\psi^{\mu}$;

- Supersimetria

As transformações de supersimetria são dadas por:

$$
\begin{aligned}
\delta X^{\mu}=\bar{\xi} \psi^{\mu} & \delta \psi^{\mu}=-i\left(\Gamma^{\alpha} \xi\right)\left(\partial_{\alpha} X^{\mu}-\bar{\psi}^{\mu} \chi_{\alpha}\right) \\
\delta e_{\alpha}^{a}=-2 i\left(\bar{\xi} \Gamma^{a} \chi_{\alpha}\right) & \delta \chi_{\alpha}=D_{\alpha} \xi
\end{aligned}
$$

Verifica-se que a álgebra de supersimetria é satisfeita apenas quando são utilizadas as equações de movimento e as simetrias acima descritas.

As três primeiras simetrias listadas implicam que a teoria construída sobre a ação $S_{R N S}$ é superconforme e, junto à supersimetria local e à simetria de Lorentz local, são responsáveis pela fixação das componentes de $e_{\alpha}^{a}$ e $\chi_{\alpha}$ :

Simetrias Bosônicas 2 simetrias de reparametrização, 1 simetria de Weyl e 1 simetria de Lorentz local. Permitem fixar completamente as 4 componentes da zweibein. Como foi feito na página 5 , escolhe-se: $e_{a}^{\alpha}=\delta_{a}^{\alpha}$, ou seja, a métrica plana $\eta^{\alpha \beta}$. 
Simetrias Fermiônicas 2 supersimetrias locais e 2 SFL, fixando as 4 componentes do espinor de RaritaSchwinger. Escolhe-se: $\chi_{\alpha}=0$.

O calibre acima é denominado calibre superconforme e, ao fixá-lo, há duas consequências diretas. Como no caso bosônico, as equações de movimento de $e_{\alpha}^{a}$ e $\chi_{\alpha}$ devem ser impostas como vínculos das soluções de $X^{\mu}$ e $\psi^{\mu}$. A outra consequência é o sacrifício da supersimetria local. Ao fixar o campo de Rarita-Schwinger, a teoria passa a possuir apenas a supersimetria global e a ação resultante é

$$
S_{R N S}^{\prime}=-\left(\frac{T}{2}\right) \int\left\{\partial^{\alpha} X \cdot \partial_{\alpha} X+i \bar{\psi} \cdot \Gamma^{\alpha} \partial_{\alpha} \psi\right\} d^{2} \sigma
$$

\subsection{Equações de Movimento e Soluções}

Tomando a representação de Majorana

$$
\Gamma^{0}=\left(\begin{array}{cc}
0 & -1 \\
1 & 0
\end{array}\right), \quad \Gamma^{1}=\left(\begin{array}{cc}
0 & 1 \\
1 & 0
\end{array}\right)
$$

e deixando explícitas as componentes espinoriais de $\psi^{\mu}=\left(\begin{array}{c}\psi_{\overline{-}}^{\mu} \\ \psi_{+}^{\mu}\end{array}\right)$, a ação (3.2) é reescrita como

$$
S_{R N S}^{\prime}=-\left(\frac{T}{2}\right) \int\left\{\partial^{\alpha} X \cdot \partial_{\alpha} X-2 i \psi_{+} \cdot \partial_{-} \psi_{+}-2 i \psi_{-} \cdot \partial_{+} \psi_{-}\right\} d^{2} \sigma .
$$

Aqui foram consideradas as coordenadas $\sigma^{ \pm}=\tau \pm \sigma, \operatorname{com} \partial_{ \pm}=\frac{1}{2}\left(\partial_{\tau} \pm \partial_{\sigma}\right)$.

As equações de movimento são, portanto:

$$
\partial_{+} \psi_{-}^{\mu}=0, \quad \partial_{+} \partial_{-} X^{\mu}=0, \quad \partial_{-} \psi_{+}^{\mu}=0 .
$$

As soluções de $X^{\mu}(\tau, \sigma)$ são idênticas àquelas do capítulo 2 dadas por (2.6) e (2.7). As soluções fermiônicas são dadas por:

$$
\begin{aligned}
\psi_{-}^{\mu}(\tau, \sigma) & =\sum_{m} c_{m}^{\mu} e^{-i m(\tau-\sigma)}, \\
\psi_{+}^{\mu}(\tau, \sigma) & =\sum_{m} \tilde{c}_{m}^{\mu} e^{-i m(\tau+\sigma)}
\end{aligned}
$$

A condição de Majorana impõe uma relação entre os modos positivos e negativos das soluções, a saber $c_{-m}^{\mu}=c_{m}^{\mu \dagger}$ e $\tilde{c}_{-m}^{\mu}=\tilde{c}_{m}^{\mu \dagger}$.

Procedendo de forma análoga ao caso bosônico, as soluções das equações de movimento devem ser ajustadas pelas condições de contorno. As coordenadas do espaço-alvo seguem a mesma discussão anterior. A fixação das condições de contorno dos campos fermiônicos é mais sutil e dá origem à setores ${ }^{3}$ diferentes na teoria. O termo de superfície associado é

$$
\int d \tau\left[\left.\left(\psi_{-} \cdot \delta \psi_{-}-\psi_{+} \cdot \delta \psi_{+}\right)\right|_{\sigma=\pi}-\left.\left(\psi_{-} \cdot \delta \psi_{-}-\psi_{+} \cdot \delta \psi_{+}\right)\right|_{\sigma=0}\right]=0
$$

\footnotetext{
${ }^{3}$ Ao discutir o espectro quântico da teoria na seção 3.4 o termo setor ficará mais claro, designando que tipo de solução dá origem à bósons ou férmions no espaço-tempo.
} 
Considerando cordas abertas, os termos entre parênteses acima devem anular-se de forma independente. Para que isso ocorra, a condição $\psi_{+}^{\mu}= \pm \psi_{-}^{\mu}$ deve ser satisfeita em cada um dos extremos. Verifica-se que apenas o sinal relativo entre os extremos é importante, de modo que será considerado $\psi_{+}^{\mu}(\tau, 0)=\psi_{-}^{\mu}(\tau, 0)$. Assim, há duas soluções possíveis:

- Condição de contorno de Ramond $(\mathrm{R}): \psi_{+}^{\mu}(\tau, \pi)=\psi_{-}^{\mu}(\tau, \pi)$.

Pelas equações (3.4) e (3.5), obtem-se:

$$
\psi_{ \pm}^{\mu}(\tau \pm \sigma)=\frac{l_{s}}{\sqrt{2}} \sum_{m \in \mathbb{Z}} d_{m}^{\mu} e^{-i m(\tau \pm \sigma)}
$$

- Condição de contorno de Neveu-Schwarz (NS): $\psi_{+}^{\mu}(\tau, \pi)=-\psi_{-}^{\mu}(\tau, \pi)$.

As soluções que satisfazem à essa condição são dadas por:

$$
\psi_{ \pm}^{\mu}(\tau \pm \sigma)=\frac{l_{s}}{\sqrt{2}} \sum_{r \in \mathbb{Z}+\frac{1}{2}} b_{r}^{\mu} e^{-i r(\tau \pm \sigma)}
$$

Há ainda a possibilidade de considerar condições diferentes para componentes diferentes. Isso claramente viola a simetria de Lorentz, além de trazer algumas implicações extremamente indesejáveis na teoria quântica, como a dificuldade de definir um estado fundamental.

É possível que uma transformação de supersimetria altere as condições de contorno, o que é certamente prejudicial. Para evitar isso, o parâmetro de supersimetria deve ter suas componentes vinculadas.

$$
\delta \psi_{-}^{\mu}=-\xi_{+} \partial_{-} X_{R}^{\mu} \quad \delta \psi_{+}^{\mu}=\xi_{-} \partial_{+} X_{L}^{\mu}
$$

Para cordas abertas de extremos livres, por exemplo, a condição sobre o parâmetro de supersimetria é $\xi_{-}=-\xi_{+}$.

Para cordas fechadas, a condição de contorno relaciona os dois extremos de acordo com $\psi^{\mu}(\tau, \sigma)=$ $\pm \psi^{\mu}(\tau, \sigma+\pi)$. Esse tipo de condição gera o dobro de setores da corda aberta (R-R, NS-NS, R-NS e NS-R) e as soluções são dadas por:

$$
\begin{array}{ll}
\psi_{+}^{\mu}(\tau+\sigma)=l_{s} \sum_{m \in \mathbb{Z}} \tilde{d}_{m}^{\mu} e^{-2 i m(\tau+\sigma)} & R \\
\psi_{+}^{\mu}(\tau+\sigma)=l_{s} \sum_{r \in \mathbb{Z}+\frac{1}{2}} \tilde{b}_{r}^{\mu} e^{-2 i r(\tau+\sigma)} & N S
\end{array}
$$

As soluções $\psi_{-}^{\mu}(\tau-\sigma)$ são similares, apenas substituindo os modos de Fourier esquerdos pelos seus correspondentes direitos: $\tilde{b}_{r}^{\mu} \rightarrow b_{r}^{\mu}$ para o setor NS; e $\tilde{d}_{m}^{\mu} \rightarrow d_{m}^{\mu}$ para o setor R.

As equações de movimento restantes, uma vez fixado o calibre superconforme, dão origem aos vínculos de super Virasoro:

- Tensor energia-momento (associado à equação de movimento da zweibein):

$$
T_{\alpha \beta} \propto \partial_{\alpha} X \cdot \partial_{\beta} X+\frac{i}{4} \bar{\psi} \cdot \Gamma_{\alpha} \partial_{\beta} \psi+\frac{i}{4} \bar{\psi} \cdot \Gamma_{\beta} \partial_{\alpha} \psi-\operatorname{traço~}=0
$$

- Supercorrente (associada à equação de movimento do campo de Rarita-Schwinger):

$$
J_{\alpha}=\left(\Gamma^{\beta} \Gamma_{\alpha} \psi\right) \cdot \partial_{\beta} X=0
$$


Embora o índice espinorial esteja implícito na equação, $J_{\alpha}$ possui em princípio 4 componentes. Entretanto, devido novamente à propriedade (B.3), $\Gamma^{\alpha} J_{\alpha}=0$, o que deixa apenas duas componentes independentes:

$$
\begin{aligned}
& J_{+}=\psi_{+} \cdot \partial_{+} X \\
& J_{-}=\psi_{-} \cdot \partial_{-} X .
\end{aligned}
$$

Assim, $X^{\mu}(\tau, \sigma)$ e $\psi^{\mu}(\tau, \sigma)$ devem satisfazer às condições acima para serem soluções válidas, que extremizam a ação RNS.

\subsection{Cargas Conservadas e Geradores de Super Virasoro}

As cargas conservadas na folha-mundo são geradas pelas seguintes correntes:

$$
\begin{aligned}
P_{\alpha}^{\mu}=T \partial_{\alpha} X^{\mu} & \rightarrow \text { Simetria de Translação no Espaço-Tempo. } \\
J_{\alpha}^{\mu \nu}=T\left(X^{\mu} \partial_{\alpha} X^{\nu}-X^{\nu} \partial_{\alpha} X^{\mu}+i \bar{\psi}^{\mu} \Gamma_{\alpha} \psi^{\nu}\right) & \rightarrow \text { Simetria de Lorentz. } \\
T_{\alpha \beta} & \rightarrow \text { Simetria de Translação na Folha-Mundo. } \\
J_{\alpha} & \rightarrow \text { Supersimetria. }
\end{aligned}
$$

A partir das soluções obtidas na seção anterior são construídos os geradores das simetrias citadas. Como o procedimento para a corda aberta e para a corda fechada é similar, apenas a primeira será utilizada para ilustrar tais resultados ${ }^{4}$.

- Setor R:

Os geradores de Lorentz são dados por:

$$
J^{\mu \nu}=x^{\mu} p^{\nu}-x^{\nu} p^{\mu}-i \sum_{n=1}^{\infty} \frac{1}{n}\left(\alpha_{-n}^{\mu} \alpha_{n}^{\nu}-\alpha_{-n}^{\nu} \alpha_{n}^{\mu}\right)-i \sum_{n=1}^{\infty}\left(d_{-n}^{\mu} d_{n}^{\nu}-d_{-n}^{\nu} d_{n}^{\mu}\right)-\frac{i}{2}\left[d_{0}^{\mu}, d_{0}^{\nu}\right] .
$$

O último termo, como será visto, corresponde exatamente aos geradores de Lorentz em uma transformação de espinores do espaço-tempo.

Os modos de Fourier do tensor energia-momento junto aos modos da supercorrente fornecem os geradores de super Virasoro.

$$
T_{++}=\sum_{m \in \mathbb{Z}} L_{m} e^{-i m(\tau+\sigma)}, \quad J_{+}=\sum_{m \in \mathbb{Z}} F_{m} e^{-i m(\tau+\sigma)} .
$$

Nessas expressões, $L_{m}=L_{m}^{(B)}+L_{m}^{(R)}$, tais que

e

$$
\begin{aligned}
& L_{m}^{(B)}=\frac{1}{2} \sum_{n \in \mathbb{Z}} \alpha_{-n} \cdot \alpha_{n+m}, \\
& L_{m}^{(R)}=\frac{1}{2} \sum_{n \in \mathbb{Z}} n d_{-n} \cdot d_{n+m},
\end{aligned}
$$

$$
F_{m}=\sum_{n \in \mathbb{Z}} \alpha_{-n} \cdot d_{n+m}
$$

\footnotetext{
${ }^{4}$ No resto do capítulo essa postura será adotada, sem perda de generalidade. Quando necessário, a distinção entre cordas abertas e fechadas será devidamente enfatizada.
} 
- Setor NS:

Os geradores de Lorentz são dados por:

$$
J^{\mu \nu}=x^{\mu} p^{\nu}-x^{\nu} p^{\mu}-i \sum_{n=1}^{\infty} \frac{1}{n}\left(\alpha_{-n}^{\mu} \alpha_{n}^{\nu}-\alpha_{-n}^{\nu} \alpha_{n}^{\mu}\right)-i \sum_{r=\frac{1}{2}}^{\infty}\left(b_{-r}^{\mu} b_{r}^{\nu}-b_{-r}^{\nu} b_{r}^{\mu}\right) .
$$

Os geradores de super Virasoro são obtidos de forma idêntica àqueles do setor R.

$$
T_{++}=\sum_{m=-\infty}^{\infty} L_{m} e^{-i m(\tau+\sigma)}, \quad J_{+}=\sum_{r \in \mathbb{Z}+\frac{1}{2}} G_{r} e^{-i r(\tau+\sigma)} .
$$

Também nessas expressões, $L_{m}=L_{m}^{(B)}+L_{m}^{(N S)}$, em que

$$
L_{m}^{(N S)}=\frac{1}{2} \sum_{r \in \mathbb{Z}+\frac{1}{2}} r b_{-r} \cdot b_{r+m},
$$

e

$$
G_{r}=\sum_{n \in \mathbb{Z}} \alpha_{-n} \cdot b_{n+r} .
$$

Nota-se claramente nos geradores de super Virasoro de ambos os setores que o termo de mistura dos modos bosônicos e fermiônicos provem da supercorrente e que suas contribuições ao tensor energia-momento são independentes.

A hamiltoniana $H$ da supercorda aberta é obtida a partir da equação de conservação do tensor energia-momento $\partial_{\alpha} T^{\alpha \beta}$, ou seja, é a carga conservada dada por

$$
H=\int_{0}^{\pi} d \sigma T^{00}=L_{0} .
$$

Ao expandir $T_{++}$e $J_{+}$em séries de Fourier, as condições (3.9) e (3.10) implicam que as soluções clássicas devem satisfazer $L_{m}=F_{m}=G_{r}=0$ para todo $m$ e $r$. Em outras palavras, cada modo de Fourier deve anular-se independentemente, constituindo uma forma alternativa de visualizar os vínculos de super Virasoro. Novamente, $L_{0}=0$ implica uma fórmula para a massa da supercorda. Para o setor $\mathrm{R}$,

$$
M^{2}=\frac{2}{l_{s}^{2}} \sum_{n=1}^{\infty}\left(\alpha_{-n} \cdot \alpha_{n}+n d_{-n} \cdot d_{n}\right)
$$

e, para o setor NS,

$$
M^{2}=\frac{2}{l_{s}^{2}} \sum_{n=1}^{\infty} \alpha_{-n} \cdot \alpha_{n}+\frac{2}{l_{s}^{2}} \sum_{r=\frac{1}{2}}^{\infty} r b_{-r} \cdot b_{r}
$$

Também aqui, o espectro de massa é contínuo e não é positivo definido. Como no caso bosônico, essa irregularidade manisfesta-se na teoria quântica, com estados que não possuem norma positiva definida. Isso será visto a seguir.

\subsection{Quantização Canônica Covariante}

No capítulo anterior foi utilizada uma simetria residual da ação da corda bosônica que permitia impor de forma muito conveniente os vínculos de Virasoro. Entretanto, ao escolher tal abordagem perde-se pelo 
caminho uma parte muito interessante da teoria, envolvendo a álgebra de Virasoro. No formalismo RNS há também uma simetria residual que permite esse tipo de atalho ao espectro físico, mas, por completeza, esta seção trata da quantização canônica explicitamente covariante, mostrando rapidamente a álgebra de Super Virasoro e algumas implicações básicas.

O procedimento canônico promove os modos de oscilação das soluções (2.11), (3.4) e (3.5) a operadores. As relações de comutação para os osciladores bosônicos são idênticas às do capítulo anterior.

Os campos espinoriais anticomutam e o procedimento canônico parte da relação $\left\{\psi^{\mu}(\tau, \sigma), \psi^{\nu}\left(\tau, \sigma^{\prime}\right)\right\}=$ $\frac{1}{T} \eta^{\mu \nu} \delta\left(\sigma-\sigma^{\prime}\right)$ para obter a álgebra dos osciladores fermiônicos:

$$
\begin{aligned}
\left\{b_{r}^{\mu}, b_{s}^{\nu}\right\} & =\delta_{r+s, 0} \eta^{\mu \nu} \\
\left\{d_{m}^{\mu}, d_{n}^{\nu}\right\} & =\delta_{m+n, 0} \eta^{\mu \nu} .
\end{aligned}
$$

Introduzindo o ordenamento normal ${ }^{5}$, a transição da teoria clássica para a teoria quântica insere constantes de ordenamento $a_{R}$ e $a_{N S}$, associadas à cada um dos setores. Apenas os geradores $L_{0}$ são afetados e, consequentemente, os operadores de massa sofrem um desvio. Definindo os operadores número

$$
N_{\alpha}=\sum_{n=1}^{\infty} \alpha_{-n}^{i} \alpha_{n}^{i}, \quad N_{b}=\sum_{r=\frac{1}{2}}^{\infty} r b_{-r}^{i} b_{r}^{i}, \quad N_{d}=\sum_{n=1}^{\infty} n d_{-n}^{i} d_{n}^{i}
$$

obtem-se:

$$
\begin{aligned}
M_{R}^{2} & =\frac{2}{l_{s}^{2}}\left\{N_{\alpha}+N_{d}-a_{R}\right\}, \\
M_{N S}^{2} & =\frac{2}{l_{s}^{2}}\left\{N_{\alpha}+N_{b}-a_{N S}\right\} .
\end{aligned}
$$

Resta agora determinar como construir os estados da teoria. A partir das relações (3.16) e (3.17), são definidas as álgebras de criação de cada setor, de tal forma que as excitações são obtidas a partir da atuação de modos bosônicos e fermiônicos em um estado fundamental adequadamente definido.

$\mathrm{O}$ estado fundamental do setor NS será representado por $|0, p\rangle_{N S}$. Autoestado do operador de momento, é aniquilado pelos modos positivos tanto bosônicos quanto fermiônicos. Uma vez que cada modo de criação é um vetor no espaço-tempo e não há degenerescência, as excitações de $|0, p\rangle_{N S}$ são bosônicas.

A definição do estado fundamental do setor $\mathrm{R},|0, p\rangle_{R}$, é mais sutil. Isso porque $\left[M_{R}^{2}, d_{0}^{\mu}\right]=0$. $\mathrm{O}$ fato de o modo zero do setor $\mathrm{R}$ comutar com o operador de massa cria uma degenerescência no estado fundamental. Como $\left\{d_{0}^{\mu}, d_{0}^{\nu}\right\}=\eta^{\mu \nu}$ assemelha-se a álgebra das matrizes de Dirac $\left(\Gamma^{\mu} \rightarrow \sqrt{2} d_{0}^{\mu}\right)$, há uma sugestão implícita de que o estado fundamental pertença a uma representação espinorial em $D$ dimensões. Portanto, este será representado por $|0, A, p\rangle_{R}$, em que $A$ é o índice espinorial. Tal estado é, por construção, aniquilado pelos modos positivos e a aplicação do modo zero fica associada à multiplicação por uma matriz de Dirac

$$
d_{0}^{\mu}|0, p\rangle_{R} \rightarrow \frac{1}{\sqrt{2}} \Gamma_{A B}^{\mu}|0, B, p\rangle_{R} .
$$

As excitações no setor $R$ tem caráter fermiônico, pois os modos de criação são bosônicos e o estado fundamental é um férmion no espaço-tempo.

Uma vez que o primeiro passo na construção dos estados da teoria foi dado, é hora de preocupar-se com os vínculos de super Virasoro. O espectro de estados gerado pela atuação aleatória de modos de criação nos estados fundamentais de ambos os setores da teoria não corresponde ao seu espectro físico. Um exemplo disso é a existência de estados de norma negativa:

\footnotetext{
${ }^{5}$ Naturalmente, o ordenamento de osciladores anticomutantes deve levar sua estatística em conta. Por exemplo, $: b_{r}^{\mu} b_{s}^{\nu}$ : é igual a $b_{r}^{\mu} b_{s}^{\nu}$ para $r<s$ e, do contrário, é igual a $-b_{s}^{\nu} b_{r}^{\mu}$.
} 


$$
\left(\alpha_{-m}^{0}|0, p\rangle_{R}\right)^{\dagger}\left(\alpha_{-m}^{0}|0, p\rangle_{R}\right)=\eta^{00}\langle 0, p \mid 0, p\rangle_{R}<0 .
$$

Isso acontece porque as condições (3.9) e (3.10) não foram impostas no nível quântico. Classicamente, tais vínculos exigem que cada um dos geradores de super Virasoro anule-se independentemente. Na teoria quântica essa condição é, de certo modo, relaxada. Por consistência, é necessário que apenas os modos de super Virasoro positivos aniquilem os estados físicos:

- setor R:

$$
\begin{gathered}
L_{m}|R\rangle=0, \quad m>0, \\
F_{m}|R\rangle=0, \quad m \geq 0, \\
\left(L_{0}-a_{R}\right)|R\rangle=0 .
\end{gathered}
$$

- setor NS:

$$
\begin{gathered}
L_{m}|N S\rangle=0, \quad m>0, \\
G_{r}|N S\rangle=0, \quad r>0, \\
\left(L_{0}-a_{N S}\right)|N S\rangle=0 .
\end{gathered}
$$

As condições acima são conhecidas como condições de concha de massa, uma vez que remetem às equações de movimento da zweibein e do campo de Rarita-Schwinger. Em particular, a condição $F_{0}|R\rangle=0$ é similar à equação de Dirac e dá origem à equação de Dirac-Ramond:

$$
\left(p \cdot \Gamma+\frac{2 \sqrt{2}}{l_{s}} \sum_{n \neq 0} \alpha_{-n} \cdot d_{n}\right)|R\rangle=0 .
$$

O critério de consistência citado no parágrafo anterior é obtido pela análise da álgebra dos geradores de super Virasoro, definida independentemente para cada setor da teoria. Com certa paciência, é possível mostrar que:

- setor R:

$$
\begin{aligned}
{\left[L_{m}, L_{n}\right] } & =(m-n) L_{m+n}+\frac{D}{8} m^{3} \delta_{m+n} \\
{\left[L_{m}, F_{n}\right] } & =\left(\frac{m}{2}-n\right) F_{m+n} \\
\left\{F_{m}, F_{n}\right\} & =2 L_{m+n}+\frac{D}{2} m^{2} \delta_{m+n} .
\end{aligned}
$$

- setor NS

$$
\begin{aligned}
{\left[L_{m}, L_{n}\right] } & =(m-n) L_{m+n}+\frac{D}{8} m\left(m^{2}-1\right) \delta_{m+n} \\
{\left[L_{m}, G_{r}\right] } & =\left(\frac{m}{2}-r\right) G_{m+r} \\
\left\{G_{r}, G_{s}\right\} & =2 L_{r+s}+\frac{D}{2}\left(r^{2}-\frac{1}{4}\right) \delta_{r+s} .
\end{aligned}
$$


Os termos nas expressões acima que envolvem a dimensão do espaço-tempo $D$ são chamados de cargas centrais, e são característicos da teoria quântica.

Agora é bastante simples entender as restrições de modos positivos em (3.19) e (3.20). Aplicando, por exemplo, o gerador $L_{-m}$ na primeira condição de (3.19), obtem-se:

$$
L_{-m} L_{m}|R\rangle=\left\{L_{m} L_{-m}+\left[L_{-m}, L_{m}\right]\right\}|R\rangle=\left\{2 m L_{0}+L_{m} L_{-m}+\frac{D}{8} m^{3}\right\}|R\rangle=0 .
$$

Pela terceira condição, $L_{o}|R\rangle=a_{R}|R\rangle$, de modo que:

$$
L_{m} L_{-m}|R\rangle=-\left\{2 m a_{R}+\frac{D}{8} m^{3}\right\}|R\rangle
$$

Como $a_{R}$ é uma constante e não depende de $m$, a condição $L_{-m}|R\rangle=0$ não é compatível com a álgebra.

Há ainda uma consequência muito interessante da álgebra do setor R. Sabe-se que $\left\{F_{0}, F_{0}\right\}=2 L_{0}$, implicando que, pela segunda condição de (3.19), $L_{0}|R\rangle=0$. Ou seja, a constante de ordenamento $a_{R}$ é nula. Esse raciocínio não pode ser estendido para a determinação de $a_{N S}$, uma vez que os geradores da supercorrente no setor NS não possuem modo zero.

Tendo introduzido a álgebra de super Virasoro e visto algumas de suas implicações no espectro de estados físicos, é interessante analisar sua origem de um ponto de vista mais crítico, entender por que existem essas relações entre os geradores se os calibres já foram escolhidos, fixando as simetrias presentes na ação RNS. As relações (3.22) e (3.23) indicam que ainda existem simetrias residuais na ação (3.2), que deixam o calibre escolhido invariante. Sua existência permite abordar a teoria quântica de uma maneira familiar, como será visto na próxima seção.

\subsection{O Calibre do Cone de Luz}

As simetrias da ação (3.1) permitem a escolha do calibre superconforme, facilitando a obtenção das soluções clássicas dos campos que a constituem. Como no caso bosônico, há simetrias residuais e estas permitem a imposição dos vínculos de super Virasoro de modo explícito.

Os passos para a fixação da simetria residual na teoria RNS não são tão simples como na corda bosônica:

- em primeiro lugar, são obtidas as soluções $X^{\mu}(\tau, \sigma), \psi^{\mu}(\tau, \sigma), e_{a}^{\alpha}=\delta_{a}^{\alpha}$ e $\chi_{\alpha}=0$;

- em seguida, são realizadas transformações de supersimetria local intercaladas com transformações de SFL, mantendo apenas o modo zero da componente $\psi^{+}=\left(\psi^{0}+\psi^{D-1}\right) / \sqrt{2}$. A simetria fermiônica local é responsável por restaurar o calibre $\chi_{\alpha}=0$ antes de cada transformação de supersimetria. Necessariamente,

$$
\partial_{ \pm} \xi=-i \Gamma_{ \pm} \chi
$$

A condição acima é obtida pelas simetrias descritas na página 19 . $\xi$ é o parâmetro das transformações de supersimetria e $\chi$ é o parâmetro da SFL;

- $\operatorname{com} \psi^{+}$fixado, escolhe-se $X^{+}(\tau, \sigma)=x^{+}+l_{s}^{2} p^{+} \tau$. Isso é possível pois a reparametrização na folha-mundo é balanceada pelo reescalonamento de Weyl e a simetria de Lorentz local, processo análogo àquele da corda bosônica, descrito na seção 2.5;

- por fim, como requisito de consistência, verifica-se que o calibre do cone de luz é invariante por transformações globais de supersimetria. 
É importante notar que as simetrias residuais não comutam, de modo que a sequência definida acima é necessária para a fixação do calibre do cone de luz.

Impondo, então, os vínculos de super Virasoro, as componentes $X^{-}$e $\psi^{-}$são escritas em função das componentes transversais $X^{i}$ e $\psi^{i}$, com $i=1, \ldots, D-2$ :

$$
\begin{aligned}
\partial_{ \pm} X^{-} & =\frac{1}{p^{+}}\left[\left(\partial_{ \pm} X^{i}\right)^{2}+\frac{i}{2} \psi_{ \pm}^{i} \cdot \partial_{ \pm} \psi_{ \pm}^{i}\right] \\
\psi_{ \pm}^{-} & =\frac{2}{p^{+}} \psi_{ \pm}^{i} \cdot \partial_{ \pm} X^{i} .
\end{aligned}
$$

Para o setor NS, $\psi^{+}=0$ e, para o setor $\mathrm{R}, \psi^{+}=d_{0}^{+}$. A demonstração das igualdades acima é trivial para o setor NS. No caso do setor R basta lembrar que $\left(d_{0}^{+}\right)^{2}=0$. Dessa forma, os vínculos de super Virasoro são satisfeitos automaticamente pelas soluções e a teoria pode ser quantizada no calibre do cone de luz, sendo descrita em termos dos modos bosônicos e fermiônicos transversais.

Embora esse calibre facilite a obtenção do espectro físico, a quebra da simetria de Lorentz (através da escolha de direções privilegiadas no espaço-tempo) pode introduzir anomalias na teoria quântica. Também a supercorda RNS fica livre de tais anomalias, desde que a dimensão do espaço-tempo e a constante de ordenamento $a_{N S}$ tenham valores específicos.

O primeiro estado excitado do setor NS, $b_{-r}^{i}|0\rangle_{N S}$, pertence à uma representação do grupo $S O(D-2)$. Para que tal estado seja um multipleto de Lorentz, o desvio de massa deve ser $a_{N S}=1 / 2$. Desse modo, ao analisar a origem da constante de ordenamento,

$$
\frac{1}{2} \sum_{m=1}^{\infty}\left[\alpha_{-m}^{i}, \alpha_{m}^{i}\right]-\frac{1}{2} \sum_{r=\frac{1}{2}}^{\infty} r\left\{b_{-r}^{i}, b_{r}^{i}\right\}=-\frac{(D-2)}{2}\left\{\sum_{m=1}^{\infty} m-\sum_{r=\frac{1}{2}}^{\infty} r\right\}=\frac{(D-2)}{2}\left(\frac{1}{12}+\frac{1}{24}\right)=a_{N S},
$$

obtem-se $D=10$. Requerendo a simetria de Lorentz no nível quântico, analogamente ao que foi feito no caso bosônico (cujo cálculo está mostrado no apêndice A), esse resultado é confirmado rigorosamente.

Até agora tudo parece muito promissor. A introdução da supersimetria na folha-mundo ajuda a reduzir o número de dimensões necessárias para uma teoria quântica que preserve a simetria de Lorentz e possui férmions e bósons do espaço-tempo no seu espectro, duas grandes vantagens sobre a teoria bosônica. Entretanto, há ainda dois detalhes incômodos e ambos estão associados essencialmente ao setor NS.

O primeiro está relacionado à diferença entre as constantes de ordenamento de cada setor. O setor $\mathrm{R}$ não possui a supersimetria quebrada, e as contribuições de ordenamento (energias de ponto zero) associadas aos modos bosônicos e fermiônicos são opostas. O mesmo não acontece no setor NS. Como já foi apontado no texto, as soluções espinoriais do setor NS não possuem o modo zero e os geradores obtidos pela conservação da supercorrente são nulos. Assim, as condições de contorno de Neveu-Schwarz quebram a supersimetria. Daí vem a discrepância encontrada, que está intimamente relacionada ao segundo detalhe incômodo, o táquion, discutido (e resolvido) ao analisar o espectro de massa, na próxima seção.

\subsection{Análise do Espectro de Massa}

Uma vez observado o poder de uma teoria supersimétrica, um espectro de estados que possua tal propriedade é muito bem vindo. Obviamente, até agora essa correspondência não foi atingida entre os setores da supercorda RNS, aberta ou fechada. Basta lembrar que o táquion está presente apenas no setor NS. 
Há, entretanto, uma maneira bastante eficiente de truncar o espectro da supercorda RNS de modo a retirar de forma consistente o tão indesejado táquion, conhecida como projeção GSO [7]. Apesar de não ser obtida de primeiros princípios, pode ser justificada completamente ao considerar a teoria em interação. Sua origem não será aqui discutida, apenas a sua estrutura e suas implicações na obtenção do espectro físico.

\subsubsection{Projeção GSO}

O ingrediente fundamental para construir o projetor GSO é o operador de número fermiônico da folha-mundo, $\mathcal{F}$. Seu papel, como o nome sugere, é contar o número de modos fermiônicos de criação em um dado estado excitado, e é definido em cada setor como:

$$
\mathcal{F}_{N S}=\sum_{r=\frac{1}{2}}^{\infty} b_{-r}^{i} b_{r}^{i} \quad \text { e } \quad \mathcal{F}_{R}=\sum_{n=1}^{\infty} d_{-n}^{i} d_{n}^{i} .
$$

Cada estado possui um número $\mathcal{F}$ associado e isso propicia a classificação de estados segundo sua paridade fermiônica, dada pelo autovalor do operador $(-1)^{\mathcal{F}+1}$. A projeção GSO trabalha com uma generalização da paridade fermiônica, chamada paridade $\mathrm{G}$, requerendo que bósons do espaço-tempo tenham paridade +1 na folha-mundo e férmions no espaço-tempo tenham paridade -1 na folha-mundo. Para isso é necessária uma projeção coerente com o espectro físico esperado. É desejável, por exemplo, que o táquion seja eliminado e os estados não massivos mantidos, sendo associados aos bósons de calibre. Assim são construídos os projetores GSO de cada setor.

- setor NS: Para eliminar o táquion, o projetor GSO do setor NS, $\mathcal{G}_{N S}$, é dado por:

$$
\mathcal{G}_{N S}=\frac{1}{2}\left[1+(-1)^{\mathcal{F}+1}\right]
$$

A paridade G no setor NS é dada apenas em função da paridade fermiônica;

- setor R: O setor R possui uma restrição própria dos espinores, e a paridade $G$ inclui também a quiralidade. Novamente, uma teoria em interação consistente requer a condição de Weyl, o que estabelece duas possibilidades de projeção GSO no setor R. A matriz de quiralidade será representada por $\Gamma^{D}$ e está definida em dimensões arbitrárias no apêndice B. Como um espinor de Weyl é autoestado da matriz de quiralidade, os projetores do setor $\mathrm{R}$ são:

$$
\mathcal{G}_{R}^{ \pm}=\frac{1}{2}\left[1 \pm \Gamma^{D}(-1)^{\mathcal{F}+1}\right]
$$

Adequadamente construída, a projeção GSO é o critério último para definir o espectro físico a partir da escolha do calibre do cone de luz.

\subsubsection{Espectro da Corda Aberta}

O espectro da corda aberta possui um setor bosônico e um fermiônico, como foi visto.

- Setor NS:

$-N_{\alpha}+N_{b}=N_{(N S)}=\frac{1}{2}$. Como o táquion é eliminado pela projeção GSO, o primeiro estado físico do setor NS é dado por $b_{-\frac{1}{2}}^{i}|0\rangle_{N S}$. Possuindo 8 direções transversais, é identificado com o fóton em $D=10$; 
$-N_{(N S)}=\frac{3}{2}$. O primeiro estado massivo do setor NS é obtido pela atuação dos seguintes modos no estado fundamental: $\alpha_{-1}^{i} b_{-\frac{1}{2}}^{j}, b_{-\frac{3}{2}}^{i}$ e $b_{-\frac{1}{2}}^{i} b_{-\frac{1}{2}}^{j} b_{-\frac{1}{2}}^{k}$. Possuindo $64+8+56$ graus de liberdade, pertence à uma representação do grupo $S O(9)$.

- setor R:

$-N_{\alpha}+N_{d}=N_{(R)}=0$. O estado fundamental corresponde a um espinor em 10 dimensões, sendo representado por $|0, A\rangle_{R}$. Em princípio, a representação mínima de tal espinor deve possuir 32 componentes complexas. Entretanto, há algumas condições que restringem esse número: a condição de Majorana; a equação de Dirac-Ramond, ou, de forma equivalente, a escolha do calibre do cone de luz; e a projeção GSO, que impõe a condição de Weyl. Cada uma delas reduz pela metade o número de graus de liberdade inicial, deixando apenas 8 graus independentes;

- $N_{(R)}=1$. Esse é o primeiro nível massivo, representado pelos estados $\alpha_{-1}^{i}|0, A\rangle_{R}$ e $d_{-1}^{i}|0, A\rangle_{R}$ que, pela projeção GSO, possuem quiralidade oposta. Ao todo, são $64+64$ graus de liberdade.

Em ambos os setores a torre de massa é infinita mas o espectro massivo não será discutido além disso.

\subsubsection{Espectro da Corda Fechada}

O espectro das cordas fechadas é dividido nos 4 setores mencionados na página 21 . Os estados físicos são construídos à partir de um produto direto entre estados criados a partir de modos direitos e esquerdos e toda a análise feita até agora para as cordas abertas de extremos livres é facilmente estendida considerando tais modos separadamente. Cada um dos setores possui uma condição de nivelamento similar àquela obtida no caso bosônico devido ao vínculo entre os modos zero das componentes $X_{R}^{-}\left(\sigma^{-}\right)$ e $X_{L}^{-}\left(\sigma^{+}\right): N_{(N S)}=\tilde{N}_{(N S)} ; N_{(R)}=\tilde{N}_{(R)} ; N_{(N S)}-1 / 2=\tilde{N}_{(R)} ;$ e $N_{(R)}=\tilde{N}_{(N S)}-1 / 2$.

- setor NS-NS: O primeiro estado excitado do setor possui massa nula e é representado por

$$
b_{-\frac{1}{2}}^{i}|0\rangle_{N S} \otimes \tilde{b}_{-\frac{1}{2}}^{j}|\tilde{0}\rangle_{N S}
$$

Pertence à representação dos tensores de segunda ordem de $S O(8)$, possuindo $1+28+35$ graus de liberdade. Sua identificação é análoga ao seu equivalente na corda bosônica, correspondendo ao dílaton, à 2-forma de Kalb-Ramond e ao gráviton, respectivamente.

Para os três setores restantes existe a preocupação com a quiralidade da projeção GSO e há duas possibilidades para tal. Uma em que são feitas projeções de quiralidade oposta e outra com quiralidades iguais. A primeira é denominada teoria de supercordas tipo $I I$-A, enquanto a última é identificada como tipo II-B. O setor NS-NS de ambas é idêntico. Os setores restantes são:

- setor R-R: junto ao setor NS-NS, o setor R-R constitui o setor bosônico da supercorda fechada. O operador de massa é dado por $M^{2}=\frac{8}{l_{s}^{2}} N_{(R)}$ e os estados não massivos possíveis são:

$$
\begin{array}{ll}
|0,-\rangle_{R} \otimes|\tilde{0},+\rangle_{R} & \\
|0,+\rangle_{R} \otimes|\tilde{0},-\rangle_{R} & \text { II-A } \\
|0,+\rangle_{R} \otimes|\tilde{0},+\rangle_{R} & \\
|0,-\rangle_{R} \otimes|\tilde{0},-\rangle_{R} & \text { II-B }
\end{array}
$$


Esses estados podem ser transformados em tensores, a partir da contração dos seus índices espinoriais com matrizes de Dirac nas dimensões transversais, $\Gamma^{i}$. Devido à álgebra das matrizes, os tensores formados são totalmente antissimétricos nos índices espaciais e são identificados com $n$-formas. Pela propriedade (B.5) do apêndice, a teoria do tipo $I I$-A contem $n=1$ e $n=3$, formas diferenciais ${ }^{6}$, constituindo $8+56$ graus de liberdade. Já a teoria do tipo $I I$-B contem $n=0, n=2$ e $n=4$ formas, possuindo $1+28+35$ graus de liberdade;

- setores R-NS e NS-R: ambos os setores são fermiônicos e os estados não massivos podem ser representados por

$$
\begin{array}{ll}
|0, \pm\rangle_{R} \otimes \tilde{b}_{-\frac{1}{2}}^{i}|\tilde{0}\rangle_{N S} & \\
b_{-\frac{1}{2}}^{i}|0\rangle_{N S} \otimes|\tilde{0}, \mp\rangle_{R} & \text { II-A } \\
|0, \pm\rangle_{R} \otimes \tilde{b}_{-\frac{1}{2}}^{i}|\tilde{0}\rangle_{N S} & \\
b_{-\frac{1}{2}}^{i}|0\rangle_{N S} \otimes|\tilde{0}, \pm\rangle_{R} & \text { II-B, }
\end{array}
$$

cada um possuindo 64 graus de liberdade.

\subsubsection{Supersimetria no Espaço-Tempo?}

Ao discutir o espectro físico da teoria, foi enfatizada a contagem dos graus de liberdade. Estados não massivos em cada um dos setores, fermiônicos ou bosônicos, possuem o mesmo número de componentes independentes. Mesmo no primeiro nível massivo da corda aberta, essa igualdade é confirmada. Uma maneira de investigar isso mais a fundo é através de uma função que possa comparar o número de estados físicos em cada nível de massa. As funções para os setores R e NS são:

$$
f_{R}(w)=\sum_{n=0}^{\infty} f_{R}(n) w^{n} \quad f_{N S}(w)=\sum_{n=0}^{\infty} f_{N S}(n) w^{n} .
$$

$f_{R}(n)$ e $f_{N S}(n)$ representam o número de estados em cada setor no mesmo nível de massa $\left(M^{2} \propto n\right)$.

Antes da projeção GSO, $f_{N S}(n)$ é proporcional ao coeficiente da potência $n$-ésima de $w$ no traço do operador $w^{N_{N S}-\frac{1}{2}}$ projetado no espectro físico. Assim:

$$
f_{N S}(w)=\operatorname{tr}\left(w^{N_{N S}-\frac{1}{2}} \mathcal{G}_{N S}\right)=\frac{1}{2}\left\{\operatorname{tr}\left(w^{N_{N S}-\frac{1}{2}}\right)-\operatorname{tr}\left[w^{N_{N S}-\frac{1}{2}}(-1)^{\mathcal{F}}\right]\right\}
$$

sendo

$$
w^{N_{N S}-\frac{1}{2}}=\frac{1}{\sqrt{w}} \prod_{i=1}^{8}\left\{\left(\prod_{n=1}^{\infty} w^{\alpha_{-n}^{i} \alpha_{n}^{i}}\right)\left(\prod_{r=\frac{1}{2}}^{\infty} w^{r b_{-r}^{i} b_{r}^{i}}\right)\right\} .
$$

Calculando o traço dos elementos presentes em $f_{N S}$ :

$$
\begin{aligned}
\operatorname{tr}\left(w^{\alpha_{-n}^{i} \alpha_{n}^{i}}\right) & =1+w^{n}+w^{2 n}+\ldots \\
\operatorname{tr}\left[w^{r b_{-r}^{i} b_{r}^{i}}( \pm 1)^{b_{-r}^{i} b_{r}^{i}}\right] & =1 \pm w^{r} .
\end{aligned}
$$

O primeiro termo corresponde a uma série geométrica. Os outros são truncados na primeira potência pelo seu caráter fermiônico, em outras palavras, $b_{-r}^{i} b_{-r}^{i}|0\rangle_{N S}=0$. Desse modo:

\footnotetext{
${ }^{6} \mathrm{~A}$ condição de Weyl identifica as $n$-formas com as $(8-n)$-formas.
} 


$$
\operatorname{tr}\left(w^{N_{N S}-\frac{1}{2}}( \pm 1)^{\mathcal{F}}\right)=\frac{1}{\sqrt{w}} \prod_{i=1}^{8} \prod_{n=1}^{\infty}\left(\frac{1}{1-w^{n}}\right) \prod_{r=\frac{1}{2}}^{\infty}\left(1 \pm w^{r}\right)=\frac{1}{\sqrt{w}} \prod_{n=1}^{\infty}\left(\frac{1 \pm w^{n-\frac{1}{2}}}{1-w^{n}}\right)^{8}
$$

Portanto:

$$
f_{N S}(w)=\frac{1}{2 \sqrt{w}}\left\{\prod_{n=1}^{\infty}\left(\frac{1+w^{n-\frac{1}{2}}}{1-w^{n}}\right)^{8}-\prod_{n=1}^{\infty}\left(\frac{1-w^{n-\frac{1}{2}}}{1-w^{n}}\right)^{8}\right\} .
$$

Para o setor R, a ação da projeção GSO é essencialmente reduzir o número de graus de liberdade de 16 para 8 , uma vez que requer férmions quirais, de modo que:

$$
f_{R}(w)=\operatorname{tr}\left(w^{N_{R}} \mathcal{G}_{R}\right)=8 \prod_{n=1}^{\infty}\left(\frac{1+w^{n}}{1-w^{n}}\right)^{8}
$$

O fator 8 multiplicativo vem do número de componentes espinoriais indepedentes e a potência 8 decorre do número de direções trasnversais.

Obtidas as funções geradoras de contagem, resta comparar os dois setores. A igualdade entre as equações (3.24) e (3.25) foi demonstrada há quase dois séculos por Carl Gustav Jacob Jacobi, que as estudou no contexto das séries q-hipergeométricas.

Conclui-se que a projeção GSO, além de eliminar o táquion da teoria, possivelmente deixa um espectro físico supersimétrico, fato ilustrado pela igualdade entre os graus de liberdade bosônicos e fermiônicos em cada nível de massa.

\subsection{Comentários}

Apesar de tudo, o formalismo RNS deixa a desejar em certos aspectos. Em primeiro lugar, ao estudar a teoria em interação, o acoplamento dos campos do setor Ramond-Ramond na folha-mundo é bastante complicado. Além disso, a projeção GSO ainda não parece um procedimento natural e a origem de uma possível supersimetria no espaço-tempo é muito obscura. Isso motivou a construção de uma teoria de supercordas cuja supersimetria fosse manifesta no espaço-tempo, resolvendo os problemas citados e construindo o espectro físico, com bósons e férmions, a partir de um único estado fundamental. Esse é denominado formalismo de Green-Schwarz [8] e será estudado no próximo capítulo. 


\section{Capítulo 4}

\section{Supercorda GS}

Neste capítulo é introduzido o formalismo de Green-Schwarz das supercordas. Partindo novamente da ação da corda bosônica, a ação GS é obtida gradativamente ao considerar as possibilidades de construção de uma teoria supersimétrica, inclusive a necessidade de introdução da simetria kapa. Ao obter as equações de movimento, uma análise qualitativa e quantitativa dos graus de liberdade da ação é realizada, verificando a possibilidade de sua linearização a partir da escolha do calibre do cone de luz. A preservação das supersimetrias nas soluções clássicas é avaliada e a quantização da teoria é realizada pelo procedimento canônico, seguindo de perto o que foi feito nos capítulos anteriores. O espectro não massivo das teorias do tipo $I, I I$-A e $I I$-B é obtido e, por fim, é introduzida a dualidade T no setor fermiônico das supercordas. Os resultados do apêndice B são recorrentes na análise das estruturas espinoriais. O conteúdo deste capítulo é baseado nas referências $[3,4,6,8,9]$.

\subsection{Supersimetria no Espaço-Tempo}

Como foi evidenciado no espectro da supercorda RNS no capítulo anterior, a igualdade no número de graus de liberdade bosônicos e fermiônicos sugere a supersimetria no espaço-tempo. Dessa forma, uma nova descrição da supercorda deve envolver um espinor no espaço-tempo, além das coordenadas do espaço-alvo:

$$
X^{\mu}(\tau, \sigma) \quad \Theta^{\mathcal{A} A}(\tau, \sigma) .
$$

Aqui, $A$ é o índice espinorial e $\mathcal{A}=1, \ldots, \mathcal{N}$ identifica a supersimetria do espinor.

Tendo como guia a supercorda RNS, a teoria requer $D=10$ dimensões. Isso pode ser demonstrado também pelo formalismo GS, mas não será discutido aqui. Assim, os espinores $\Theta^{\mathcal{A}}$ possuem 32 componentes. Além disso, o espectro RNS contém férmions de Majorana e, devido à projeção GSO, são restritos ainda pela condição de Weyl, de modo que cada deles possui 16 componentes reais. No que segue, será utilizada a representação de Majorana das matrizes de Dirac, cujas propriedades principais são mostradas no apêndice $\mathrm{B}^{1}$.

As transformações de supersimetria dos campos acima são dadas por:

$$
\begin{aligned}
\delta \Theta^{\mathcal{A}} & =\xi^{\mathcal{A}}, \\
\delta X^{\mu} & =i \bar{\Theta}^{\mathcal{A}} \Gamma^{\mu} \xi^{\mathcal{A}} .
\end{aligned}
$$

\footnotetext{
${ }^{1}$ A condição combinada de Majorana-Weyl não é possível em qualquer dimensão, apenas em $D=2$ (mod 8 ). O apêndice apresenta uma discussão detalhada desta e outras propriedade das matrizes de Dirac em dimensão arbitrária.
} 
O fator imaginário na transformação de $X^{\mu}$ assegura sua realidade.

A ação (2.2) é um guia muito bem sucedido, como foi visto no capítulo anterior. Assim, uma forma razoável de construir uma ação supersimétrica no espaço-tempo é partir de uma extensão das coordenadas do espaço-alvo. O objeto definido por

$$
\Pi_{\alpha}^{\mu} \equiv \partial_{\alpha} X^{\mu}+i \bar{\Theta}^{\mathcal{A}} \Gamma^{\mu} \partial_{\alpha} \Theta^{\mathcal{A}},
$$

é o candidato mais evidente, possibilitanto a construção da seguinte generalização supersimétrica da ação (2.2) no espaço plano:

$$
S^{\prime}=-\left(\frac{T}{2}\right) \int \sqrt{-h}\left(h^{\alpha \beta} \Pi_{\alpha} \cdot \Pi_{\beta}\right) d^{2} \sigma .
$$

Pela discussão da seção 3.6, assumindo a supersimetria no espectro RNS, há uma evidência muito forte de que esta seja duplicada. Em cada setor R-NS e NS-R existe um estado de spin $\frac{3}{2}$. Estes seriam os superparceiros do gráviton, denominados gravitinos. Como são estados independentes, há, em princípio, supersimetria $\mathcal{N}=2$. Assim, apesar da elegância, a ação acima sofre de um sério problema: não há como igualar, mesmo na concha de massa, o número de graus de liberdade bosônicos e fermiônicos ao obter o espectro quântico. A extensão para um modelo supersimétrico no espaço-tempo deve incluir ao menos duas supersimetrias, o que torna uma teoria baseada somente na ação (4.3) incapaz de descrever tal espectro. Há, entretanto, uma simetria fermiônica que permite resolver esse problema perfeitamente, que é o assunto da próxima seção.

\subsection{Simetria kapa e a Ação GS}

A motivação para introduzir a simetria kapa, em particular, vem do estudo da superpartícula, que mantém tal simetria escondida em sua ação ${ }^{2}$. Apesar de ser muito similar à supersimetria, a simetria kapa apresenta uma diferença fundamental, a localidade na folha-mundo. A transformação das coordenadas fermiônicas é genericamente representada por $\delta_{\kappa} \Theta^{\mathcal{A}}$. Já as coordenadas do espaço-alvo transformam-se como

$$
\delta_{\kappa} X^{\mu}=-i \bar{\Theta}^{\mathcal{A}} \Gamma^{\mu}\left(\delta_{\kappa} \Theta^{\mathcal{A}}\right) .
$$

Considerando uma possível transformação da métrica da folha-mundo, a ação $S^{\prime}$ é modificada da seguinte forma:

$$
\delta_{\kappa} S^{\prime}=-2 T \int \sqrt{-h}\left\{h^{\alpha \beta} \Pi_{\alpha}^{\mu}\left(i \delta_{\kappa} \bar{\Theta}^{\mathcal{A}} \Gamma^{\nu} \partial_{\beta} \Theta^{\mathcal{A}}\right) \eta_{\mu \nu}+\frac{1}{4} \delta_{\kappa}\left(\sqrt{-h} h^{\alpha \beta}\right) \Pi_{\alpha} \cdot \Pi_{\beta}\right\} d^{2} \sigma
$$

A contribuição de uma outra possível parte da ação, $S^{\prime \prime}$, deve cancelar a transformação acima, bem como possuir as simetrias presentes em $S^{\prime}$, especialmente a supersimetria. Uma forma de implementar $S^{\prime \prime}$ é considerar um espaço tridimensional $\mathcal{D}$ cuja fronteira é a folha-mundo $\mathcal{M}=\partial \mathcal{D}$, utilizando algumas técnicas de geometria diferencial. Assim:

$$
S^{\prime \prime}=\int_{\mathcal{D}} \Omega_{3}=\int_{\mathcal{M}} \Omega_{2}, \quad \operatorname{com} \Omega_{3}=d \Omega_{2} .
$$

A 3-forma $\Omega_{3}$ deve ser supersimétrica e invariante de Lorentz. Para construí-la, dispõe-se de três 1-formas que possuem os requisitos necessários, a saber, $d \Theta^{1}, d \Theta^{2}$ e $\Pi^{\mu}=d X^{\mu}+i \bar{\Theta}^{\mathcal{A}} \Gamma^{\mu} d \Theta^{\mathcal{A}}$. Assim, $\Omega_{3}$ deve possuir a seguinte estrutura (o produto externo entre as formas está implícito):

$$
\Omega_{3}=c_{1} d \bar{\Theta}^{1} \Gamma^{\mu} d \Theta^{1} \Pi_{\mu}+c_{2} d \bar{\Theta}^{2} \Gamma^{\mu} d \Theta^{2} \Pi_{\mu} .
$$

\footnotetext{
${ }^{2}$ As cargas conservadas associadas à essa simetria anulam-se na concha de massa. Uma discussão detalhada é apresentada em [10] e revista rapidamente em [4].
} 
Nesta equação, $c_{i}$ são constantes a serem determinadas. Um outro possível termo que satisfaz aos requisitos necessários é $d \bar{\Theta}^{1} \Gamma^{\mu} d \Theta^{2} \Pi_{\mu}$. Há duas boas razões para dispensá-lo. A primeira delas é que a teoria deve descrever espinores de quiralidade oposta ou igual, e o bilinear formado por $d \Theta^{1}$ e $d \Theta^{2}$ anula-se para quiralidades opostas, de acordo com (B.5). Isso indica que esse termo é irrelevante para a teoria II-A. Pela dualidade $\mathrm{T}$, descrita na seção 2.7 para a corda bosônica e a ser introduzida na seção 4.7 para supercordas, a teoria $I I$-A é dual à teoria $I I$-B, o que justifica por completo sua exclusão. A segunda razão, mais prática, é que a simetria kapa é perfeitamente implementada sem o bilinear misto. Isso será demonstrado a seguir, quando, ao ignorá-la, a ação $S^{\prime \prime}$ será construída consistentemente com a contribuição kapa de $S^{\prime}$.

O primeiro teste de consistência de $\Omega_{3}$ é $d \Omega_{3}=0$, uma vez que é uma forma exata.

$$
d \Omega_{3}=i c_{1}\left(d \bar{\Theta}^{1} \Gamma^{\mu} d \Theta^{1}\right)\left(d \bar{\Theta}^{2} \Gamma_{\mu} d \Theta^{2}\right)+i c_{2}\left(d \bar{\Theta}^{2} \Gamma^{\mu} d \Theta^{2}\right)\left(d \bar{\Theta}^{1} \Gamma_{\mu} d \Theta^{1}\right) .
$$

Os termos quárticos com o mesmo espinor não aparecem devido à propriedade (B.6), fundamental ${ }^{3}$ na análise da supercorda GS. Para $d \Omega_{3}=0$, obtem-se $c_{1}=-c_{2}$.

$\Omega_{2}$ é obtido por uma integração simples:

$$
\Omega_{2}=c_{1}\left(\bar{\Theta}^{1} \Gamma^{\mu} d \Theta^{1}-\bar{\Theta}^{2} \Gamma^{\mu} d \Theta^{2}\right) d X_{\mu}+i c_{1}\left(\bar{\Theta}^{1} \Gamma^{\mu} d \Theta^{1}\right)\left(\bar{\Theta}^{2} \Gamma_{\mu} d \Theta^{2}\right) .
$$

A solução acima é dada a menos de uma 2-forma exata, que contribuiria na ação como um termo de superfície. Agora é necessário investigar a transformação de simetria kapa de $\Omega_{2}$. Para isso, a construção $\Omega_{3}=d \Omega_{2}$ é de bastante utilidade, já que a transformação da 3-forma é facilmente obtida:

$$
\begin{aligned}
\delta_{\kappa} \Omega_{3} & =c_{1} d\left(\delta_{\kappa} \bar{\Theta}^{1}\right) \Gamma^{\mu} d \Theta^{1} \Pi_{\mu}+c_{1} d \bar{\Theta}^{1} \Gamma^{\mu} d\left(\delta_{\kappa} \Theta^{1}\right) \Pi_{\mu}+c_{1} d \bar{\Theta}^{1} \Gamma^{\mu} d \Theta^{1}\left(\delta_{\kappa} \Pi_{\mu}\right) \\
& -c_{1} d\left(\delta_{\kappa} \bar{\Theta}^{2}\right) \Gamma^{\mu} d \Theta^{2} \Pi_{\mu}-c_{1} d \bar{\Theta}^{2} \Gamma^{\mu} d\left(\delta_{\kappa} \Theta^{2}\right) \Pi_{\mu}-c_{1} d \bar{\Theta}^{2} \Gamma^{\mu} d \Theta^{2}\left(\delta_{\kappa} \Pi_{\mu}\right) .
\end{aligned}
$$

Novamente, devido à propriedade (B.6),

$$
\left(d \bar{\Theta}^{1} \Gamma^{\mu} d \Theta^{1}-d \bar{\Theta}^{2} \Gamma^{\mu} d \Theta^{2}\right)\left(\delta_{\kappa} \Pi_{\mu}\right)=2\left[\left(\delta_{\kappa} \bar{\Theta}^{1}\right) \Gamma^{\mu} d \Theta^{1}-\left(\delta_{\kappa} \bar{\Theta}^{2}\right) \Gamma^{\mu} d \Theta^{2}\right] d \Pi_{\mu},
$$

o que permite escrever $\delta_{\kappa} \Omega_{3}$ como uma forma exata:

$$
\delta_{\kappa} \Omega_{3}=d\left[2 c_{1}\left(\delta_{\kappa} \bar{\Theta}^{1} \Gamma^{\mu} d \Theta^{1}-\delta_{\kappa} \bar{\Theta}^{2} \Gamma^{\mu} d \Theta^{2}\right) \Pi_{\mu}\right] .
$$

Portanto, a transformação de simetria kapa de $\Omega_{2}$ é

$$
\delta_{\kappa} \Omega_{2}=2 c_{1}\left(\delta_{\kappa} \bar{\Theta}^{1} \Gamma^{\mu} d \Theta^{1}-\delta_{\kappa} \bar{\Theta}^{2} \Gamma^{\mu} d \Theta^{2}\right) \Pi_{\mu} .
$$

Somando as contribuições das ações $S^{\prime}$ e $S^{\prime \prime}$, obtem-se:

$$
\begin{aligned}
\delta_{\kappa}\left(S^{\prime}+S^{\prime \prime}\right) & =-2 T \int \sqrt{-h}\left\{h^{\alpha \beta} \Pi_{\alpha}^{\mu}\left(i \delta_{\kappa} \bar{\Theta}^{\mathcal{A}} \Gamma_{\mu} \partial_{\beta} \Theta^{\mathcal{A}}\right)+\frac{1}{4} \delta_{\kappa}\left(\sqrt{-h} h^{\alpha \beta}\right) \Pi_{\alpha} \cdot \Pi_{\beta}\right\} d^{2} \sigma \\
& +2 c_{1} \int \sqrt{-h} \epsilon^{\alpha \beta}\left(\delta_{\kappa} \bar{\Theta}^{1} \Gamma_{\mu} \partial_{\alpha} \Theta^{1}-\delta_{\kappa} \bar{\Theta}^{2} \Gamma_{\mu} \partial_{\alpha} \Theta^{2}\right) \Pi_{\beta}^{\mu} .
\end{aligned}
$$

Aqui a notação de formas diferenciais foi substituída pela convencional, sendo $\epsilon^{\alpha \beta}=-\epsilon^{\beta \alpha}$ e $\epsilon^{01}=1$. Definindo $c_{1}=-i T$, são introduzidos os objetos

$$
P_{ \pm}^{\alpha \beta}=\frac{1}{2}\left(h^{\alpha \beta} \pm \frac{\epsilon^{\alpha \beta}}{\sqrt{-h}}\right)
$$

${ }^{3}$ De fato há uma seção, B.3, inteiramente dedicada a ela e algumas implicações básicas. 
chamados projetores de auto dualidade na folha-mundo ${ }^{4}$. Assim:

$$
\begin{aligned}
\delta_{\kappa}\left(S^{\prime}+S^{\prime \prime}\right) & =-4 T \int \sqrt{-h}\left\{\Pi_{\alpha}^{\mu} P_{-}^{\alpha \beta}\left(i \delta_{\kappa} \bar{\Theta}^{1} \Gamma_{\mu} \partial_{\beta} \Theta^{1}\right)\right\} d^{2} \sigma \\
& -4 T \int \sqrt{-h}\left\{\Pi_{\alpha}^{\mu} P_{+}^{\alpha \beta}\left(i \delta_{\kappa} \bar{\Theta}^{2} \Gamma_{\mu} \partial_{\beta} \Theta^{2}\right)\right\} d^{2} \sigma \\
& -\frac{T}{2} \int\left\{\delta_{\kappa}\left(\sqrt{-h} h^{\alpha \beta}\right) \Pi_{\alpha} \cdot \Pi_{\beta}\right\} d^{2} \sigma
\end{aligned}
$$

Em analogia com a superpartícula, discutida nas referências [11, 3, 4], espera-se que $\delta_{\kappa} \Theta^{\mathcal{A}}=\Gamma_{\mu} \Pi_{\alpha}^{\mu} \kappa^{\mathcal{A} \alpha}$, sendo $\kappa^{\mathcal{A} \alpha}$ o parâmetro das transformações kapa. Substituindo-a, obtem-se, após algumas manipulações,

$$
\begin{aligned}
\delta_{\kappa}\left(S^{\prime}+S^{\prime \prime}\right) & =2 T \int \sqrt{-h} \Pi_{\alpha}^{\mu} \Pi_{\beta}^{\nu}\left\{\partial_{\gamma} \bar{\Theta}^{1} \Gamma_{\mu} \Gamma_{\nu}\left(P_{-}^{\alpha \gamma} \kappa^{1 \beta}-P_{-}^{\beta \gamma} \kappa^{1 \alpha}\right)\right\} d^{2} \sigma \\
& +2 T \int \sqrt{-h} \Pi_{\alpha}^{\mu} \Pi_{\beta}^{\nu}\left\{\partial_{\gamma} \bar{\Theta}^{2} \Gamma_{\mu} \Gamma_{\nu}\left(P_{+}^{\alpha \gamma} \kappa^{2 \beta}-P_{+}^{\beta \gamma} \kappa^{2 \alpha}\right)\right\} d^{2} \sigma \\
& -\frac{T}{2} \int \Pi_{\alpha} \cdot \Pi_{\beta}\left\{\delta_{\kappa}\left(\sqrt{-h} h^{\alpha \beta}\right)-8 i \sqrt{-h}\left(P_{-}^{\alpha \gamma} \partial_{\gamma} \bar{\Theta}^{1} \kappa^{1 \beta}+P_{+}^{\alpha \gamma} \partial_{\gamma} \bar{\Theta}^{2} \kappa^{2 \beta}\right)\right\} d^{2} \sigma .
\end{aligned}
$$

Impondo que a ação seja invariante, é estabelecida a lei de transformação da métrica $h^{\alpha \beta}$ e o vínculo para os parâmetros $\kappa^{\mathcal{A} \alpha}$ :

$$
\begin{aligned}
\kappa^{1 \alpha} & =P_{-}^{\alpha \beta} \kappa_{\beta}^{1} \\
\kappa^{2 \alpha} & =P_{+}^{\alpha \beta} \kappa_{\beta}^{2} .
\end{aligned}
$$

Com isso obtem-se a ação GS das supercordas, na qual a supersimetria manifesta-se no espaço-tempo:

$$
\begin{aligned}
S_{G S} & =-\left(\frac{T}{2}\right) \int \sqrt{-h}\left\{h^{\alpha \beta} \Pi_{\alpha} \cdot \Pi_{\beta}\right\} d^{2} \sigma \\
& -i T \int \epsilon^{\alpha \beta}\left[\left(\bar{\Theta}^{1} \Gamma^{\mu} \partial_{\alpha} \Theta^{1}-\bar{\Theta}^{2} \Gamma^{\mu} \partial_{\alpha} \Theta^{2}\right) \partial_{\beta} X_{\mu}+i\left(\bar{\Theta}^{1} \Gamma^{\mu} \partial_{\alpha} \Theta^{1}\right)\left(\bar{\Theta}^{2} \Gamma_{\mu} \partial_{\beta} \Theta^{2}\right)\right] d^{2} \sigma .
\end{aligned}
$$

Suas simetrias são:

- Reparametrização da Folha-Mundo

- Simetria de Weyl Local

- Simetria de Poincaré

- Supersimetria no Espaço-Tempo

As transformações supersimetria dos campos presentes na ação (4.8) são dadas em (4.1). A contribuição $S^{\prime}$ é naturalmente supersimétrica. Isso ainda não foi verificado para $S^{\prime \prime}$.

$$
\begin{aligned}
\delta_{\xi} S^{\prime \prime} & \propto \int\left\{\left(\bar{\xi}^{1} \Gamma^{\mu} d \Theta^{1}-\bar{\xi}^{2} \Gamma^{\mu} d \Theta^{2}\right) d X_{\mu}+\left(\bar{\Theta}^{1} \Gamma^{\mu} d \Theta^{1}-\bar{\Theta}^{2} \Gamma^{\mu} d \Theta^{2}\right)\left(i d \bar{\Theta}^{\mathcal{A}} \Gamma^{\mu} \xi^{\mathcal{A}}\right)\right\} \\
& +i \int\left\{\left(\bar{\xi}^{1} \Gamma^{\mu} d \Theta^{1}\right)\left(\bar{\Theta}^{2} \Gamma_{\mu} d \Theta^{2}\right)+\left(\bar{\Theta}^{1} \Gamma^{\mu} d \Theta^{1}\right)\left(\bar{\xi}^{2} \Gamma_{\mu} d \Theta^{2}\right)\right\}
\end{aligned}
$$

${ }^{4}$ Algumas propriedades:

$$
P_{ \pm}^{\alpha \gamma} h_{\gamma \rho} P_{ \pm}^{\rho \beta}=P_{ \pm}^{\alpha \beta} \quad P_{ \pm}^{\alpha \gamma} h_{\gamma \rho} P_{\mp}^{\rho \beta}=0 \quad P_{ \pm}^{\alpha \gamma} P_{ \pm}^{\rho \beta}=P_{ \pm}^{\rho \gamma} P_{ \pm}^{\alpha \beta}
$$


A primeira contribuição entre parênteses é uma forma exata, correspondendo a um termo de superfície. Rearranjando a transformação:

$$
\delta_{\xi} S^{\prime \prime} \sim \int d^{2} \sigma \epsilon^{\alpha \beta}\left\{\left(\bar{\Theta}^{1} \Gamma^{\mu} \partial_{\beta} \Theta^{1}\right)\left(\bar{\xi}^{1} \Gamma^{\mu} \partial_{\alpha} \Theta^{1}\right)+\left(\bar{\Theta}^{2} \Gamma^{\mu} \partial_{\alpha} \Theta^{2}\right)\left(\bar{\xi}^{2} \Gamma_{\mu} \partial_{\beta} \Theta^{2}\right)\right\} .
$$

Pela discussão final do apêndide $\mathrm{B}$, os termos dentro das chaves são derivadas totais, o que demonstra que $\delta_{\xi} S^{\prime \prime}=0$ a menos de termos de superfície. Isso ocorre porque $S^{\prime \prime}$ é obtida a partir da integração de uma 3-forma supersimétrica;

- Simetria kapa

É a base da construção da ação GS. As transformações kapa dos campos são:

$$
\begin{aligned}
\delta_{\kappa} \Theta^{\mathcal{A}} & =\Gamma_{\mu} \Pi_{\alpha}^{\mu} \kappa^{\mathcal{A} \alpha} \\
\delta_{\kappa} X^{\mu} & =-i \Pi_{\alpha}^{\nu}\left(\bar{\Theta}^{\mathcal{A}} \Gamma^{\mu} \Gamma_{\nu} \kappa^{\mathcal{A} \alpha}\right) \\
\delta_{\kappa}\left(\sqrt{-h} h^{\alpha \beta}\right) & =8 i \sqrt{-h}\left(P_{-}^{\alpha \gamma} \partial_{\gamma} \bar{\Theta}^{1} \kappa^{1 \beta}+P_{+}^{\alpha \gamma} \partial_{\gamma} \bar{\Theta}^{2} \kappa^{2 \beta}\right)
\end{aligned}
$$

\subsection{Cargas Conservadas e Geradores de Simetrias do Espaço- Tempo}

As cargas associadas à simetrias do espaço-tempo são facilmente obtidas pelo procedimento de Noether.

- Simetria de Translação $\left(\delta X^{\mu}=a^{\mu} ; \delta \Theta^{\mathcal{A}}=0\right)$ :

$$
P^{\mu, \alpha}=T \sqrt{-h}\left\{h^{\alpha \beta} \partial_{\beta} X^{\mu}+2 i P_{-}^{\alpha \beta}\left(\bar{\Theta}^{1} \Gamma^{\mu} \partial_{\beta} \Theta^{1}\right)+2 i P_{+}^{\alpha \beta}\left(\bar{\Theta}^{2} \Gamma^{\mu} \partial_{\beta} \Theta^{2}\right)\right\} .
$$

- Simetria de Lorentz $\left(\delta X^{\mu}=a^{\mu}{ }_{\nu} X^{\nu} ; \delta \Theta^{\mathcal{A}}=\frac{1}{4} a_{\mu \nu} \Gamma^{\mu \nu} \Theta^{\mathcal{A}}\right)$ :

$$
\begin{aligned}
J^{\mu \nu, \alpha} & =X^{\mu} P^{\nu, \alpha}-X^{\nu} P^{\mu, \alpha} \\
& +\frac{i}{2} h_{\rho \lambda} P^{\lambda, \alpha}\left(\bar{\Theta}^{1} \Gamma^{\mu \rho \nu} \Theta^{1}+\bar{\Theta}^{2} \Gamma^{\mu \rho \nu} \Theta^{2}\right) \\
& +i \frac{T}{2} \bar{\Theta}^{1} \Gamma^{\mu \rho \nu} \Theta^{1} \epsilon^{\alpha \beta}\left(\partial_{\beta} X_{\rho}+\bar{\Theta}^{1} \Gamma_{\rho} \partial_{\beta} \Theta^{1}\right) \\
& -i \frac{T}{2} \bar{\Theta}^{2} \Gamma^{\mu \rho \nu} \Theta^{2} \epsilon^{\alpha \beta}\left(\partial_{\beta} X_{\rho}+\bar{\Theta}^{2} \Gamma_{\rho} \partial_{\beta} \Theta^{2}\right) .
\end{aligned}
$$

- Supersimetria $\left(\delta X^{\mu}=i \bar{\Theta}^{\mathcal{A}} \Gamma^{\mu} \xi^{\mathcal{A}} ; \delta \Theta^{\mathcal{A}}=\xi^{\mathcal{A}}\right)$ :

$$
\begin{aligned}
Q^{1 \alpha} & =T\left\{\sqrt{-h} P_{+}^{\alpha \beta}\left(\partial_{\beta} X_{\mu}+i \bar{\Theta}^{2} \Gamma_{\mu} \partial_{\beta} \Theta^{2}\right)-\frac{2 i}{3} \epsilon^{\alpha \beta} \bar{\Theta}^{1} \Gamma_{\mu} \partial_{\beta} \Theta^{1}\right\} \Gamma^{\mu} \Theta^{1}, \\
Q^{2 \alpha} & =T\left\{\sqrt{-h} P_{-}^{\alpha \beta}\left(\partial_{\beta} X_{\mu}+i \bar{\Theta}^{1} \Gamma_{\mu} \partial_{\beta} \Theta^{1}\right)+\frac{2 i}{3} \epsilon^{\alpha \beta} \bar{\Theta}^{2} \Gamma_{\mu} \partial_{\beta} \Theta^{2}\right\} \Gamma^{\mu} \Theta^{2} .
\end{aligned}
$$

A conservação das cargas é obtida a partir das equações $\partial_{\alpha}(\ldots)^{\alpha}=0$, identificadas com os geradores das simetrias do espaço-tempo. 


\subsection{Equações de Movimento e Graus de Liberdade}

As equações de movimento dos campos presentes na ação (4.8) são dadas por:

- $h^{\alpha \beta}$ :

$$
\Pi_{\alpha} \cdot \Pi_{\beta}-\frac{1}{2} h_{\alpha \beta}\left(h^{\gamma \rho} \Pi_{\gamma} \cdot \Pi_{\rho}\right)=0
$$

- $X^{\mu}$ :

$$
\partial_{\alpha}\left\{\sqrt{-h}\left[h^{\alpha \beta} \partial_{\beta} X^{\mu}+2 i P_{-}^{\alpha \beta}\left(\bar{\Theta}^{1} \Gamma^{\mu} \partial_{\beta} \Theta^{1}\right)+2 i P_{+}^{\alpha \beta}\left(\bar{\Theta}^{2} \Gamma^{\mu} \partial_{\beta} \Theta^{2}\right)\right]\right\}=0 .
$$

Como esperado, a equação de movimento das coordenadas do espaço-alvo está associada à simetria de translação, dada em (4.10).

- $\Theta^{1}$ :

$$
\Pi_{\alpha} \cdot \Gamma\left(P_{-}^{\alpha \beta} \partial_{\beta} \Theta^{1}\right)=0
$$

- $\Theta^{2}$ :

$$
\Pi_{\alpha} \cdot \Gamma\left(P_{+}^{\alpha \beta} \partial_{\beta} \Theta^{2}\right)=0 .
$$

Novamente, as simetrias de reparametrização e reescalonamento de Weyl simplificam bastante a análise das equações de movimento e suas possíveis soluções, sendo possível a escolha $h^{\alpha \beta}=\eta^{\alpha \beta}$. Desse modo, o número de graus de liberdade restantes origina-se exclusivamente das coordenadas do espaço-alvo e dos espinores:

- $X^{\mu}(\tau, \sigma)$ possui 10 componentes reais. Há ainda a simetria residual de reparametrização e a equação de movimento da métrica, (4.14), que dá origem ao vínculo de Virasoro da supercorda GS:

$$
\left(\Pi_{0}^{\mu} \pm \Pi_{1}^{\mu}\right)^{2}=0
$$

Nesse caso, o número de graus de liberdade bosônicos é 8;

- $\Theta^{\mathcal{A}}(\tau, \sigma)$ possui 16 componentes reais. Como há duas supersimetrias, o número de graus de liberdade fermiônicos é 32 .

No caso fermiônico, resta avaliar ainda quantos graus de liberdade são fixados pela simetria kapa.

Definindo $\gamma_{ \pm}=\left(\Pi_{0}^{\mu} \pm \Pi_{1}^{\mu}\right) \Gamma_{\mu}$, as equações de movimento são reescritas como:

$$
\begin{aligned}
& \gamma_{-} \partial_{+} \Theta^{1}=0 \\
& \gamma_{+} \partial_{-} \Theta^{2}=0
\end{aligned}
$$

É fácil notar que $\left(\gamma_{ \pm}\right)^{2}=0$ e $\left\{\gamma_{+}, \gamma_{-}\right\} \propto \mathbb{I}$. É possível, então, construir uma base de projeção bidimensional,

$$
\begin{aligned}
& \Pi^{2}=\eta^{\alpha \beta} \Pi_{\alpha} \cdot \Pi_{\beta}, \\
& P_{+}=-\frac{1}{2 \Pi^{2}} \gamma_{+} \gamma_{-}, \\
& P_{-}=-\frac{1}{2 \Pi^{2}} \gamma_{-} \gamma_{+},
\end{aligned}
$$

satisfazendo a $P_{+}+P_{-}=\mathbb{I}$ e $P_{+} P_{-}=P_{-} P_{+}=0$. 
Os vínculos dos parâmetros da simetria kapa dados em (4.6) e (4.7) podem ser vistos da seguinte forma:

$$
\begin{aligned}
& \eta^{\alpha \beta} \kappa_{\beta}^{1}=P_{-}^{\alpha \beta} \kappa_{\beta}^{1} \Rightarrow \kappa_{0}^{1}=\kappa_{1}^{1} \equiv \kappa^{1}, \\
& \eta^{\alpha \beta} \kappa_{\beta}^{2}=P_{+}^{\alpha \beta} \kappa_{\beta}^{2} \Rightarrow \kappa_{0}^{2}=-\kappa_{1}^{2} \equiv \kappa^{2} .
\end{aligned}
$$

Desse modo,

$$
\begin{aligned}
\delta_{\kappa} \Theta^{1} & =\gamma_{-} \kappa^{1}, \\
\delta_{\kappa} \Theta^{2} & =\gamma_{+} \kappa^{2} .
\end{aligned}
$$

Pelo caráter projetivo ${ }^{5}$ das matrizes $\gamma_{ \pm}$, as equações acima evidenciam que metade das componentes de $\kappa^{1}$ e $\kappa^{2}$ não contribuem para a transformação kapa dos espinores. Estendendo a análise, a contagem das componentes relevantes do parâmetro da transformação é a seguinte:

- inicialmente, os parâmetros $\kappa^{\mathcal{A} \alpha}$ possuem ao todo $2 \times 2 \times 16$ componentes. São duas componentes vetorias da folha-mundo, duas supersimetrias e 16 componentes espinoriais, já estabelecidas pela condição de Majorana-Weyl;

- as condições de auto dualidade (4.6) e (4.7) eliminam metade dos graus descritos acima, deixando 32 componentes independentes;

- pela construção da transformação, como em (4.20) e (4.21), apenas metade dos graus de liberdade restantes contribuem, de modo que, ao todo, a simetria kapa pode fixar 16 graus de liberdade.

Nota-se que as equações de movimento de $\Theta^{A}$ não eliminam graus de liberdade além daqueles que podem ser fixados pela simetria kapa. Isso será verificado na próxima seção. De fato, a partir deste ponto, o único modo de reduzir graus de liberdade fermiônicos é através de quebra de supersimetria. Há duas formas bastante evidentes que incluem essa possibilidade, embora ambas tenham um princípio em comum.

As equações de movimento das coordenadas do espaço-alvo permitiram, ao menos até aqui, uma construção independente de soluções que se propagam em direções opostas na folha-mundo. Em termos práticos, isso constitui uma duplicação dos graus de liberdade. Enquanto não há relação entre essas soluções, há 16 graus de liberdade bosônicos. A partir do momento em que modos direitos e esquerdos são associados há uma quebra de supersimetria. Desse modo, cordas abertas e cordas não orientadas devem possuir apenas uma supersimetria no formalismo de Green-Schwarz. Essas considerações serão aprofundadas ao obter o espectro físico. Para chegar lá, o único caminho conhecido é a fixação do calibre do cone de luz, que é estudado a seguir.

\subsection{O Calibre do Cone de Luz no Formalismo GS}

As equações de movimento (4.14)-(4.17) são muito difíceis de serem resolvidas. Sua não linearidade implica em vínculos que até hoje não foram resolvidos de forma covariante. Nesse ponto, a simetria kapa mostra o seu poder.

Motivada pela supercorda RNS, a escolha do calibre de $\Theta^{\mathcal{A}}$ (fixado pela simetria kapa) deve ser tal que a transformação de supersimetria mantenha o calibre no cone de luz, se for possível fixá-lo. Como $\delta X^{+}=-i\left(\delta \bar{\Theta}^{\mathcal{A}}\right) \Gamma^{+} \Theta^{\mathcal{A}}$, a escolha natural é $\Gamma^{+} \Theta^{\mathcal{A}}=0$. Na seção D.2 do apêndice há uma demonstração bastante simples dessa possibilidade.

\footnotetext{
${ }^{5} \mathrm{O}$ apêndice $\mathrm{D}$ discute alguns aspectos das matrizes singulares dessa forma.
} 
Resta analisar o impacto dessa escolha nas equações de movimento. Em primeiro lugar, obtem-se que $\bar{\Theta}^{\mathcal{A}} \Gamma^{\mu} \partial_{\alpha} \Theta^{\mathcal{A}}$ é diferente de zero apenas para $\mu=-$. Para $\mu=+$ a demonstração é trivial. Para o caso das dimensões transversais, $\mu=i$, basta lembrar que $\sqrt{2} \Gamma^{0}=\Gamma^{+}+\Gamma^{-}$e $\left\{\Gamma^{ \pm}, \Gamma^{i}\right\}=0$. Assim:

- $\Theta^{\mathcal{A}}$ : Para o caso dos espinores as equações de movimento são bastante simplificadas:

$$
\begin{aligned}
& \partial_{+} \Theta^{1}=0 . \\
& \partial_{-} \Theta^{2}=0 .
\end{aligned}
$$

As manipulações que levam às equações acima são diretas. Nota-se que as propriedades de auto dualidade manifestadas pelos parâmetros das transformações kapa, (4.6) e (4.7), vigoram em todo o desenvolvimento da teoria para cada um dos espinores. As equações de movimento determinam que $\Theta^{1}$ e $\Theta^{2}$ propagam-se em direções opostas na folha-mundo.

- $X^{\mu}$ : Pela equação (4.15):

$$
\partial^{\alpha} \partial_{\alpha} X^{\mu}+2 i P_{-}^{\alpha \beta} \partial_{\alpha}\left(\bar{\Theta}^{1} \Gamma^{\mu} \partial_{\beta} \Theta^{1}\right)+2 i P_{+}^{\alpha \beta} \partial_{\alpha}\left(\bar{\Theta}^{2} \Gamma^{\mu} \partial_{\beta} \Theta^{2}\right)=0 .
$$

Para $\mu=+, i$. os termos com espinores não contribuem. Para $\mu=-$, basta utilizar as equações de movimento dos espinores e a propriedade (B.4) do apêndice e verifica-se que:

$$
\partial_{+} \partial_{-} X^{\mu}=0
$$

Todas essas equações já foram estudadas nos capítulos anteriores. Devido à simetria residual de reparametrização, é possível escolher $X^{+}(\tau, \sigma)=x^{+}+l_{s}^{2} p^{+} \tau$, como desejado. Esse é o calibre do cone de luz permitindo, mais uma vez, quantizar a teoria de modo conveniente ${ }^{6}$.

Uma vez que há 32 supercargas, obtidas pela conservação da supercorrente, é possível que uma transformação de supersimetria retire o sistema do calibre do cone de luz, o que pode ser desastroso. Portanto, ao construir uma teoria supersimétrica consistente com a escolha de calibre, as transformações de supersimetria devem preservá-lo, diretamente ou não. No primeiro caso, o parâmetro da transformação satisfaz $\Gamma^{+} \xi=0$ e há 8 supercargas para cada supersimetria. No último caso, $\Gamma^{-} \xi=0$, o calibre deve ser restaurado por uma transformação de simetria kapa e uma reparametrização residual.

Ao denotar as componentes independentes de $\Theta^{\mathcal{A}}$ por $S^{\mathcal{A} a}$, com $a=1, \ldots, 8$ e fazer a identificação

$$
\Theta^{\mathcal{A}} \rightarrow \frac{1}{\sqrt{2 p^{+} \sqrt{2}}} S^{\mathcal{A}},
$$

é possível, no calibre do cone de luz, identificar uma ação classicamente equivalente à (4.8):

$$
S_{c l}=-\frac{T}{2} \int d^{2} \sigma\left[\partial_{\alpha} X^{i} \partial^{\alpha} X^{i}+2 i\left(S^{1} \partial_{+} S^{1}+S^{2} \partial_{-} S^{2}\right)\right] .
$$

A semelhança com a ação do formalismo RNS no cone de luz é notável, embora haja uma diferença básica. No formalismo RNS, os espinores (folha-mundo) pertencem a uma representação fundamental de $S O(8)$, genericamente representada por $\mathbf{8}_{v}$. No formalismo GS, aparece o grupo de cobertura Spin (8), que é simplesmente conexo, e os espinores (espaço-tempo) pertencem a uma de suas representações $\boldsymbol{8}_{s}$ ou $\mathbf{8}_{c}$, dependendo da quiralidade. Há uma simetria de trialidade entre esses grupos e todos possuem dimensão 8, o que é bastante incomum, uma vez que representações espinoriais e vetorias geralmente possuem dimensão diferente. As implicações dessa diferença serão analisadas na próxima seção, ao obter e discutir o espectro físico pelo procedimento canônico de quantização.

\footnotetext{
6“Conveniente", nesse caso, caracteriza a obtenção do espectro de estados. Obviamente, um procedimento de quantização covariante de Lorentz seria muito benquisto, tendo em vista, por exemplo, o poder das simetrias na construção da teoria em interação.
} 


\subsection{Quantização e Espectro Fundamental}

O procedimento de quantização é muito parecido com aquele do formalismo RNS. A diferença está no setor fermiônico. Nesse caso, ao fazer a transição para a teoria quântica obtem-se a relação

$$
\left\{S^{\mathcal{A} a}(\tau, \sigma), S^{\mathcal{B} b}\left(\tau, \sigma^{\prime}\right)\right\}=\frac{1}{T} \delta^{\mathcal{A B}} \delta^{a b} \delta\left(\sigma-\sigma^{\prime}\right),
$$

permitindo obter as relações de anticomutação entre os modos de Fourier das soluções.

Até aqui, a quiralidade dos espinores não foi tratada explicitamente. Espinores cuja quiralidade for positiva, pertencem à representação $\mathbf{8}_{s}$, e suas componentes serão associadas ao índice $a$. Para quiralidade negativa, o índice associado será pontuado, $\dot{a}$, pertencendo à representação espinorial $\mathbf{8}_{c}$. Desse modo, é conveniente, pela escolha do calibre do cone de luz, introduzir as matrizes de Dirac em 8 dimensões, as direções transversais, que, devido à condição de Weyl, assumem a seguinte forma:

$$
\Gamma^{i}=\left(\begin{array}{cc}
0 & \gamma_{a \dot{a}}^{i} \\
\gamma_{\dot{a} a}^{i} & 0
\end{array}\right)
$$

$\gamma^{i}$ são matrizes quadradas $8 \times 8$ que, devido a algebra de Dirac, satisfazem

$$
\gamma_{a \dot{a}}^{i} \gamma_{\dot{a} b}^{j}+\gamma_{a \dot{a}}^{j} \gamma_{\dot{a} b}^{i}=2 \delta^{i j} \delta_{a b}
$$

Naturalmente, a aplicação de uma matriz $\Gamma^{i}$ em um espinor $S$ altera sua quiralidade. A seguir são analisadas as possibilidades de construção dos estados não massivos do espectro quântico. A construção de estados excitados é análoga ao que foi feito até agora para a corda bosônica e para a supercorda RNS.

\subsubsection{Supersimetria $\mathcal{N}=2$}

Para preservar a supersimetria $\mathcal{N}=2$ no espectro, é preciso que o número de graus bosônicos fornecidos pelas coordenadas do espaço-alvo seja dobrado, como foi discutido na seção 4.4. Isso acontece apenas no caso das cordas fechadas, uma vez que as soluções $X^{\mu}(\tau, \sigma)$ são compostas de duas partes independentes, dadas em (2.8) e (2.9), cujo vínculo único é o modo zero na expansão de Fourier.

As soluções fermiônicas para cordas fechadas são dadas por

$$
\begin{aligned}
& S^{1}(\tau, \sigma)=l_{s} \sum_{m \in \mathbb{Z}} S_{m}^{1} e^{-2 i m(\tau-\sigma)}, \\
& S^{2}(\tau, \sigma)=l_{s} \sum_{m \in \mathbb{Z}} S_{m}^{2} e^{-2 i m(\tau+\sigma)}
\end{aligned}
$$

obtidas com a condição de periodicidade $S^{\mathcal{A}}(\tau, \sigma)=S^{\mathcal{A}}(\tau, \sigma+\pi)$. Pela condição de Majorana, $S_{-m}^{\mathcal{A}}=$ $\left(S_{m}^{\mathcal{A}}\right)^{\dagger}$. Promovendo os modos de Fourier clássicos a operadores, verifica-se que:

$$
\left\{S_{m}^{\mathcal{A} a}, S_{n}^{\mathcal{B} b}\right\}=\delta^{\mathcal{A B}} \delta^{a b} \delta_{m+n}
$$

O espectro de estados é construído como aquele do setor de Ramond na supercorda RNS. Devido aos vínculos de Virasoro, a componente $p^{-}$do vetor de momento é escrita em função de $p^{+}$e dos modos de Fourier nas direções transversais, de modo que o operador de massa é dado por:

$$
\frac{1}{4} l_{s}^{2} M^{2}=\sum_{n=1}^{\infty}\left\{\alpha_{-n}^{i} \alpha_{n}^{i}+\tilde{\alpha}_{-n}^{i} \tilde{\alpha}_{n}^{i}+n\left(S_{-n}^{1} S_{n}^{1}+S_{-n}^{2} S_{n}^{2}\right)\right\} .
$$


Por uma análise simples, é possível mostrar que não há a necessidade de uma constante de ordenamento, uma vez que as contribuições bosônicas e fermiônicas são opostas. A construção do estado fundamental baseia-se na simetria de trialidade mencionada acima:

$$
\begin{aligned}
& \mathbf{8}_{v} \rightarrow|i\rangle \\
& \mathbf{8}_{s} \rightarrow|a\rangle \\
& \mathbf{8}_{c} \rightarrow|\dot{a}\rangle
\end{aligned}
$$

Como $\left[M^{2}, S_{0}^{\mathcal{A}}\right]=0$ e $S_{0}^{\mathcal{A}}$ tem caráter fermiônico, os estados acima podem ser mapeados um no outro com o auxílio de (4.22). Assim, omitindo o índice de supersimetria:

$$
\begin{aligned}
|i\rangle & =\gamma_{\dot{a} a}^{i} S_{0}^{\dot{a}}|a\rangle \\
|a\rangle & =\gamma_{a \dot{a}}^{i} S_{0}^{\dot{a}}|i\rangle,
\end{aligned}
$$

$\mathrm{ou}$

$$
\begin{aligned}
|i\rangle & =\gamma_{a \dot{a}}^{i} S_{0}^{a}|\dot{a}\rangle \\
|\dot{a}\rangle & =\gamma_{\dot{a} a}^{i} S_{0}^{a}|i\rangle .
\end{aligned}
$$

Construído dessa forma, o espectro da supercorda GS é manisfestamente supersimétrico, com bósons e férmions sendo criados a partir de excitações de um único estado fundamental.

\subsubsection{Cordas Fechadas Tipo $I I-\mathrm{A}$}

Esse tipo de supercordas é constituído por dois espinores de quiralidade oposta, $S^{1}$ pertence à representação $\mathbf{8}_{s}$ enquanto $S^{2}$ pertence à representação $\mathbf{8}_{c}$. O conteúdo do estado fundamental é obtido a partir de uma decomposição tensorial do produto

$$
\left(\boldsymbol{8}_{v}+\mathbf{8}_{s}\right)_{R} \otimes\left(\mathbf{8}_{v}+\mathbf{8}_{c}\right)_{L}
$$

Portanto:

- $\boldsymbol{8}_{v} \otimes \boldsymbol{8}_{v}$ corresponde ao setor NS-NS obtido na subseção 3.6.3. Representando o dílaton, o gráviton e a 2-forma de Kalb-Ramond, possui ao todo 64 graus de liberdade.

- $\mathbf{8}_{s} \otimes \mathbf{8}_{c}$ corresponde ao setor R-R da supercorda RNS. Como o estado é uma combinação de quiralidades opostas, representado por $|a ; \dot{a}\rangle$, basta analisar as contrações com as matrizes $\gamma_{a \dot{a}}^{i}$ para obter as represetações $\mathbf{8}_{v}$ :

- $\gamma_{a \dot{a}}^{i}|a ; \dot{a}\rangle$ é um vetor de $\mathbf{8}_{v}$, associado a uma 1-forma;

- $\gamma_{a \dot{b}}^{i} \gamma_{\dot{b} b}^{j} \gamma_{b \dot{a}}^{k}|a ; \dot{a}\rangle$ é uma representação tensorial de ordem 3 totalmente antissimétrica de $\mathbf{8}_{v}$, desiganda por $56_{t}$, associada a uma 3 -forma;

- $\mathbf{8}_{v} \otimes \mathbf{8}_{c}$ e $\mathbf{8}_{s} \otimes \mathbf{8}_{v}$ são os parceiros supersimétricos das combinações acima, somando 128 graus de liberdade. 


\subsubsection{Cordas Fechadas Tipo $I I-\mathrm{B}$}

Nesse caso os espinores possuem a mesma quiralidade e, consequentemente, o espectro gerado é diferente da supercorda $I I$-A. O conteúdo do estado fundamental é obtido a partir de uma decomposição tensorial do produto

$$
\left(\mathbf{8}_{v}+\mathbf{8}_{s}\right)_{R} \otimes\left(\mathbf{8}_{v}+\mathbf{8}_{s}\right)_{L}
$$

Portanto:

- $\boldsymbol{8}_{v} \otimes \mathbf{8}_{v}$ é idêntico à teoria $I I-\mathrm{A}$.

- $\boldsymbol{8}_{s} \otimes \boldsymbol{8}_{s}$ é o setor R-R da teoria $I I$-B. O estado é uma combinação de duas quiralidades iguais, representado por $|a ; b\rangle$ e a decomposição tensorial é obtida através da contração com um número par de matrizes $\gamma_{a \dot{a}}^{i}$ :

- $|a ; a\rangle$ é um escalar, associado a uma 0-forma;

- $\gamma_{a \dot{b}}^{i} \gamma_{\dot{b} b}^{j}|a ; b\rangle$ é uma representação tensorial de ordem 2 antissimétrica de $\mathbf{8}_{v}$, associada a uma 2-forma. Possui 28 graus de liberdade;

- $\gamma_{a \dot{b}}^{i} \gamma_{\dot{b} c}^{j} \gamma_{c \dot{c}}^{k} \gamma_{\dot{c} b}^{l}|a ; b\rangle$ é uma representação tensorial de ordem 4 totalmente antissimétrica de $\boldsymbol{8}_{v}$, associada a uma 4-forma. Possui, em princípio, 70 graus de liberdade. Entretanto, a condição de Weyl torna-a auto dual, de modo que há 35 graus de liberdade independentes.

- $\boldsymbol{8}_{v} \otimes \mathbf{8}_{s}$ e $\mathbf{8}_{s} \otimes \mathbf{8}_{v}$ são os parceiros supersimétricos das combinações acima, somando 128 graus de liberdade.

Neste ponto cabe uma observação sobre o espectro acima construído. Se existe alguma simetria que relaciona os setores NS-NS e R-R das cordas fechadas, ela deve ocorrer na supercorda $I I$-B. Nota-se aqui que estão presentes, em ambos os setores, uma 2-forma e uma 0-forma, embora a 4-forma esteja presente apenas no setor R-R.

\subsubsection{Supersimetria $\mathcal{N}=1$}

Como foi discutido, espera-se que uma construção supersimétrica consistente que possua um vínculo mais forte que aquele citado na subseção anterior ${ }^{7}$ leve à quebra de uma das supersimetrias. Os dois vínculos imediatos nessa consideração são a condição de contorno de cordas abertas e a projeção de inversão de extremos na corda fechada. Essas são conhecidas como as supercordas tipo $I$.

\subsubsection{Cordas Abertas}

Ao considerar os termos de superfície a serem fixados pelas condições de contorno de cordas abertas, os dois extremos devem anular-se de forma independente. Concluiu-se que existem 8 graus de liberdade bosônicos. Quanto às soluções fermiônicas, há uma relação clara entre os espinores com índice de supersimetria diferentes. Em cada extremo da corda, a condição $S^{1}\left(\delta S^{1}\right)=S^{2}\left(\delta S^{2}\right)$ deve ser satisfeita, em analogia com o que foi discutido na seção 3.2. Isso pode ocorrer de duas maneiras:

\footnotetext{
${ }^{7}$ Em outras palavras, um vínculo que associe todos os modos de Fourier direitos e esquerdos, ao invés de apenas um.
} 
- $S^{1}=-S^{2}$

Essa condição quebra todas as supersimetrias da teoria. A primeira supersimetria é quebrada pela associação entre os espinores. A segunda supersimetria é quebrada porque soluções que satisfazem esse tipo de condição de contorno não possuem o modo zero na expansão de Fourier, o que desequilibra, de forma evidente, as soluções clássicas bosônicas e fermiônicas.

- $S^{1}=S^{2}$

As soluções das equações de movimento com essa condição de contorno são dadas por:

$$
\begin{aligned}
& S^{1}(\tau, \sigma)=\frac{l_{s}}{\sqrt{2}} \sum_{m \in \mathbb{Z}} S_{m} e^{-i m(\tau-\sigma)}, \\
& S^{2}(\tau, \sigma)=\frac{l_{s}}{\sqrt{2}} \sum_{m \in \mathbb{Z}} S_{m} e^{-i m(\tau+\sigma)} .
\end{aligned}
$$

Nota-se aqui que os modos de Fourier das soluções são iguais. Pela condição de Majorana, $S_{-m}=$ $\left(S_{m}\right)^{\dagger}$. Promovendo os modos de Fourier clássicos à operadores, obtem-se:

$$
\left\{S_{m}^{a}, S_{n}^{b}\right\}=\delta^{a b} \delta_{m+n}
$$

Desse ponto em diante, será considerada a supercorda aberta com supersimetria $\mathcal{N}=1$.

A construção do espectro de estados é direta, conforme discutido para cordas fechadas. Novamente, devido aos vínculos de Virasoro é possível construir o operador de massa a partir dos modos de oscilação transversais:

$$
\frac{1}{2} l_{s}^{2} M^{2}=\sum_{n=1}^{\infty}\left\{\alpha_{-n}^{i} \alpha_{n}^{i}+n S_{-n} S_{n}\right\}
$$

O estado fundamental é uma combinação bosônica e fermiônica, a primeira pertencendo a representação fundamental de $\mathbf{8}_{v}$ e a última a uma representação fundamental de $\mathbf{8}_{s}$ :

- $\boldsymbol{8}_{v}$ é o equivalente do setor NS na supercorda RNS. Os estados são representados por $|i\rangle$ e estão associados ao fóton em 10 dimensões.

- $\boldsymbol{8}_{s}$ é o equivalente do setor R na supercorda RNS. Os estados são representados por $|\dot{a}\rangle$ e estão associados ao fotino, superparceiro do fóton, em 10 dimensões.

A relação entre os estados é idêntica àquela dada em (4.26).

\subsubsection{Cordas Fechadas Não Orientadas}

O espectro das cordas não orientadas não será aqui obtido e a discussão subsequente é incluída apenas para ilustrar uma alternativa de redução do número de supersimetrias.

Se é requisitado que as soluções de corda fechada dadas em (2.8), (2.9), (4.23) e (4.24) possuam a simetria $\sigma \rightarrow \pi-\sigma$, os modos das soluções que se propagam em direções opostas na folha-mundo são 
vinculados:

$$
\begin{aligned}
X_{R}^{\mu}(\tau, \sigma) & =\frac{x^{\mu}}{2}+l_{s} \alpha_{0}^{\mu}(\tau-\sigma)+i \frac{l_{s}}{2} \sum_{m \neq 0} \frac{1}{m} \alpha_{m}^{\mu} e^{-2 i m(\tau-\sigma)} \\
X_{L}^{\mu}(\tau, \sigma) & =\frac{x^{\mu}}{2}+l_{s} \alpha_{0}^{\mu}(\tau+\sigma)+i \frac{l_{s}}{2} \sum_{m \neq 0} \frac{1}{m} \alpha_{m}^{\mu} e^{-2 i m(\tau+\sigma)}, \\
S^{1}(\tau, \sigma) & =l_{s} \sum_{m \in \mathbb{Z}} S_{m} e^{-2 i m(\tau-\sigma)} \\
S^{2}(\tau, \sigma) & =l_{s} \sum_{m \in \mathbb{Z}} S_{m} e^{-2 i m(\tau+\sigma)} .
\end{aligned}
$$

A supersimetria é quebrada, de modo que as soluções acima possuem apenas supersimetria $\mathcal{N}=1$.

\subsection{Dualidade T e as Supercordas}

Quando a dualidade T discutida na seção 2.7 é estendida ao setor fermiônico, qualquer possível ação nas coordenadas espinoriais deve ser consistente com as transformações de supersimetria. Para uma transformação de supersimetria que não preserva o calibre da simetria $k a p a\left(\Gamma^{+} \xi^{\mathcal{A}} \neq 0\right)$ :

$$
\begin{aligned}
& \delta X_{R}^{I}=i \bar{\Theta}^{1} \Gamma^{I} \xi^{1} \\
& \delta X_{L}^{I}=i \bar{\Theta}^{2} \Gamma^{I} \xi^{2}
\end{aligned}
$$

Aqui o índice $I$ representa uma das dimensões compactificadas. Definindo o operador de dualidade $\mathrm{T}$ e lembrando que $T\left(\delta X_{R}^{I}\right)=-\delta X_{R}^{I}$ e $T\left(\delta X_{L}^{I}\right)=\delta X_{L}^{\mu I}$ :

$$
\begin{aligned}
& T\left[\bar{\Theta}^{1} \Gamma^{I} \xi^{1}\right]=-\bar{\Theta}^{1} \Gamma^{I} \xi^{1}, \\
& T\left[\bar{\Theta}^{2} \Gamma^{I} \xi^{2}\right]=+\bar{\Theta}^{2} \Gamma^{I} \xi^{2} .
\end{aligned}
$$

Para construir a operação da dualidade nos espinores, basta associar $T$ a uma matriz quadrada $32 \times 32$, ou seja:

$$
T\left(\Theta^{\mathcal{A}}\right)_{A}=T_{A B}^{(\mathcal{A})} \Theta_{B}^{\mathcal{A}}
$$

Assim, são estabelecidas as seguintes relações (omitindo os índices espinoriais $A$ ):

$$
\begin{gathered}
T^{(1) T} \Gamma^{0} \Gamma^{I} T^{(1)}=-\Gamma^{0} \Gamma^{I} \Rightarrow T^{(1)}=\Gamma^{I}, \\
T^{(2) T} \Gamma^{0} \Gamma^{I} T^{(2)}=\Gamma^{0} \Gamma^{I} \Rightarrow T^{(2)}=\mathbb{I} .
\end{gathered}
$$

Portanto, $T\left(\Theta^{1}\right)=\Gamma^{I} \Theta^{1}$ e $T\left(\Theta^{2}\right)=\Theta^{2}$.

\subsubsection{Cordas Fechadas}

Uma implicação muito interessante da dualidade $\mathrm{T}$ na teoria de supercordas fechadas, é que existe a possibilidade de alteração da quiralidade de um dos espinores, ou seja, as teorias do tipo $I I$-A e $I I$-B são duais. Denotando as componentes independentes de $\Theta^{\mathcal{A}}$ por $S^{\mathcal{A} a}$, com $a=1, \ldots, 8$, a dualidade T pode ser resumida nas duas situações abaixo: 
- quando é aplicada a um número de dimensões ímpar,

$$
T\left(S^{1 a}\right)=\gamma_{a \dot{b}}^{I_{1}} \ldots \gamma_{b \dot{c}}^{I_{2 n-1}} S^{1 \dot{c}}
$$

há alteração da quiralidade de $S^{1}$, enquanto $S^{2}$ permanece. A teoria $I I-\mathrm{A}(\mathrm{B})$ é dual a teoria $I I-\mathrm{B}(\mathrm{A})$.

- para um número de dimensões par,

$$
T\left(S^{1 a}\right)=\gamma_{a \dot{b}}^{I_{1}} \ldots \gamma_{\dot{c} b}^{I_{2 n}} S^{1 b}
$$

não há alteração de quiralidade e as teorias são auto duais.

Há uma certa ambiguidade na ordem de multiplicação das matrizes $\gamma^{I}$ que, ao final, corresponde a uma troca global de sinal ( $\left.S^{1} \rightarrow-S^{1}\right)$. Entretanto, essa é uma simetria da ação GS, de modo que o sinal não é importante.

\subsubsection{Cordas Abertas}

No caso de cordas abertas, já foi visto que a dualidade $\mathrm{T}$ mapeia condições de contorno de extremos livres em extremos fixos e vice-versa.

A análise para o setor fermiônico pode, em princípio, parecer infrutífera. Já foi visto que as condições de contorno aplicadas às soluções espinoriais estabalecem um vínculo entre os espinores, induzindo à conclusão de que possuem a mesma quiralidade. Entretanto é possível mostrar que não há ambiguidades na extensão direta do que foi visto para a corda fechada. As condições de contorno $S^{1}\left(\delta S^{1}\right)=S^{2}\left(\delta S^{2}\right)$ continuam invariantes sobre a transformação $T\left(\Theta^{\mathcal{A}}\right)$ definida na subseção anterior.

$$
\begin{aligned}
& T\left(S^{1 a}\right)=\gamma_{a \dot{b}}^{I_{1}} S^{1 \dot{b}} \\
& T\left(S^{2 a}\right)=S^{2 a} .
\end{aligned}
$$

Assim, a dualidade $\mathrm{T}$ pode inverter a quiralidade relativa dos espinores da supercorda tipo $I$, embora tal operação não reflita em nada nos observáveis físicos, visto que as soluções $S^{1}$ e $S^{2}$ continuam vinculadas, preservando a supersimetria $\mathcal{N}=1$ e mantendo, por exemplo, o espectro da massa quântico.

\subsubsection{Constantes de Acoplamento}

Um ponto omitido até aqui e que merece certa atenção é o entendimento da dualidade $\mathrm{T}$ na teoria em interação, ao menos sua validade perturbativa. Ao analisar esse aspecto, é interessante observar a compactificação em uma ação efetiva. Em geral, tal ação apresenta-se como

$$
S_{e f}=\frac{1}{g_{s}^{2}} \int d^{10} x \mathcal{L}
$$

sendo $g_{s}$ a constante de acoplamento da teoria. Ao considerar, por exemplo, o setor NS-NS da teoria $I I$-A com uma dimensão compactificada de raio $R$, obtem-se:

$$
S_{e f}=\frac{2 \pi R}{g_{s}^{2}} \int d^{9} x \mathcal{L}_{N S-N S} .
$$


Como foi visto na seção 2.7, tal ação deve ser equivalente àquela da teoria $I I$-B compactificada em uma dimensão de raio

$$
\tilde{R}=\frac{l_{s}^{2}}{2 R}
$$

uma vez que tal setor é igual em ambas. Nesse caso:

$$
S_{e f}=\frac{2 \pi \tilde{R}}{\tilde{g}_{s}^{2}} \int d^{9} x \mathcal{L}_{N S-N S}=\frac{2 \pi R}{g_{s}^{2}} \int d^{9} x \mathcal{L}_{N S-N S} .
$$

Portanto, é estabelecida a relação entre as constantes de acoplamento entre duas teorias T duais:

$$
\tilde{g}_{s}=g_{s} \frac{l_{s}}{\sqrt{2} R} .
$$

Essa relação será utilizada na seção 5.2 ao estudar a relação entre as tensões das D p-branas.

\subsection{Comentários}

O formalismo de Green-Schwarz foi, durante muito tempo, a ferramenta mais adequada para um estudo consistente das supercordas, seu espectro e suas interações. A supersimetria manifesta no espaçotempo contribui de forma muito evidente nos cálculos da teoria em interação, entretanto sua importância é, em parte, apagada pelo sacrifício realizado na obtenção do espectro quântico: a simetria de Lorentz não é manifesta. Há alguns anos foi introduzido o formalismo de espinores puros [12], pelo qual a supercorda é quantizada com ambas as simetrias manifestas, supersimetria e simetria de Lorentz. Justamente por isso, essa nova proposta tem se mostrado bastante promissora, embora os cálculos envolvidos ainda sejam bastante intrincados. Sua equivalência com o formalismo de GS tem sido verificada abundantemente.

No final do capítulo foi discutida a dualidade $T$ na supercorda e, com ela, foram obtidas as ferramentas necessárias para estudar a dinâmica dos objetos extensos introduzidos no capítulo da corda bosônica, as branas. No próximo capítulo discute-se a dinâmica das p-branas e das D-branas no limite de baixas energias, onde apenas os campos não massivos são acoplados. Também nesse limite, são introduzidas as ações de supergravidade, com uma discussão básica de suas propriedades. 


\section{Capítulo 5}

\section{Branas e Supergravidade}

Neste capítulo são introduzidas as ações efetivas no limite de baixas energias associadas a teoria de supercordas. Discute-se o acoplamento das p-branas com os campos de calibre e determina-se a condição de Dirac-Nepomechie-Teiltelboim, que determina a quantização das cargas elétrica e magnética da brana. Em seguida, é analisada a construção de uma ação efetiva para a D p-brana, inferindo a presença do campo de calibre sobre a brana. Partindo de uma construção genérica, as transformações de supersimetria e simetria kapa desse "novo" campo são determinadas requerendo a validade da álgebra de supersimetria em sua forma mais simples, determinando a ação no limite de baixas energias da $\mathrm{D} p$-brana. Em seguida, são introduzidas as ações de supergravidade em $D=11$, associada a teoria $\mathrm{M}$, e em $D=10$, associadas ao espectro não massivo das supercordas $I I$-A e $I I$-B.

\section{$5.1 p$-branas}

As $p$-branas, como introduzido na seção 2.1, são objetos com $p$ dimensões espaciais que se propagam no espaço-tempo gerando superfícies $p+1$ dimensionais, o volume-mundo, que é o componente fundamental da sua descrição dinâmica, (2.1). Analogamente ao caso da partícula relativística, espera-se que o acoplamento com um campo de calibre seja através de uma $(p+1)$-forma. Por exemplo, para $p=1$, o acoplamento seria análogo àquele descrito na equação (2.21), em que o campo de Kalb-Ramond acopla-se à corda. De forma geral, o termo de acoplamento é

$$
S_{a e}=Q_{p} \int A_{p+1}
$$

Aqui é utilizada a notação compacta de formas diferenciais, $Q_{p}$ é a carga da forma $A_{p+1}$, denominada, por abuso de linguagem, carga elétrica da $p$-brana. Seguindo essa construção, é possível definir o acoplamento magnético de uma $p$-brana:

$$
S_{a m}=\tilde{Q}_{p} \int \star A_{p+1}
$$

Nesse caso, $\tilde{Q}_{p}$ é a carga magnética da brana e a operação $\star$, definida por

$$
(\star A)_{\mu_{1} \ldots \mu_{D-p}}=\frac{1}{(p !) \sqrt{|g|}} e_{\mu_{1} \ldots \mu_{D-p}}^{\nu_{1} \ldots \nu_{p}} A_{\nu_{1} \ldots \nu_{p}},
$$


é denominada operação Hodge estrela, mapeando uma $p$-forma em uma $(D-p)$-forma, ambas sobre uma variedade de dimensão $D$. Na definição, $g$ é o determinante da métrica $D$ dimensional e $e_{\mu_{1} \ldots \mu_{D}}$ é o tensor completamente antissimétrico nesse espaço.

Naturalmente, ao introduzir o acoplamento entre as $p$-branas e os campos de calibre, é necessário preocupar-se também com sua dinâmica. O termo cinético de $A_{p+1}$ é construído a partir do tensor do campo, $F_{p+2}=d A_{p+1}$ :

$$
S_{c i n} \sim \int F_{p+2}\left(\star F_{p+2}\right) .
$$

Quando se admite a existência de cargas magnéticas, é possível obter a condição de Dirac-NepomechieTeitelboim (DNT), uma extensão da condição de quantização de carga de Dirac, como pode ser visto a seguir.

Partindo de uma generalização da Lei de Gauss, obtem-se:

$$
\begin{aligned}
Q_{p} & =\int_{S^{D-p-2}} \star F_{p+2}, \\
\tilde{Q}_{p} & =\int_{S^{p+2}} F_{p+2} .
\end{aligned}
$$

Nessas identidades, $S^{D-p-2}$ é uma esfera que envolve a $p$-brana.

Assumindo, sempre em analogia com o eletromagnetismo, que a mudança de fase associada à função de onda da $p$-brana seja

$$
\delta=i Q_{p} \int_{\gamma} A_{p+1},
$$

em que $\gamma$ é a superfície $(p+1)$ dimensional descrita na propagação da brana, obtem-se, pelo teorema de Stokes:

$$
\delta=i Q_{p} \int_{\Sigma} F_{p+2}
$$

Aqui, $\Sigma$ é o volume cuja fronteira é $\gamma$. Como este não é univocamente definido, é possível escolher um volume $\Sigma^{\prime}$ alternativo cuja fronteira também é $\gamma$.

$$
\delta^{\prime}=i Q_{p} \int_{\Sigma^{\prime}} F_{p+2}
$$

A mudança de fase na função de onda é insensível a essa escolha, de modo que $\delta-\delta^{\prime}=i 2 \pi n$, com $n \in \mathbb{Z}$. Como a superfície $\Sigma-\Sigma^{\prime}$ é equivalente a $S^{p+2}$, verifica-se que a condição de invariância de fase e a equação (5.3) implicam em

$$
Q_{p} \tilde{Q}_{p}=2 \pi n .
$$

Essa é a condição DNT que associa as cargas elétrica e a carga magnética de uma brana.

Isso conclui uma rápida introdução à dinâmica de acoplamento das $p$-branas com as formas $A_{p+1}$. Na próxima seção, discute-se a construção da ação das branas de Dirichlet como uma generalização supersimétrica e kapa simétrica da ação das $p$-branas no limite de baixas energias.

\section{$5.2 \quad \mathrm{D} p$-branas}

Para o regime de baixas energias (mais especificamente, $l_{s} \rightarrow 0$ ), apenas os estados fundamentais da teoria são relevantes para uma possível descrição das D-branas. Isso é equivalente a dizer que nesse limite 
apenas os estados não massivos são criados e, consequentemente, constituem os únicos estados da corda aberta que podem interagir com a brana.

A composição da ação de uma D-brana deve levar em conta suas coordenadas do espaço-alvo, $X^{\mu}(\sigma)$, em que $\sigma^{\alpha}$ é a parametrização do volume-mundo, com $\alpha=0, \ldots, p$, e as coordenadas fermiônicas $\Theta^{\mathcal{A} A}(\sigma)$, com $\mathcal{A}$ representando o número de supersimetrias e $A$ o índice espinorial. Em $D=10, A=1, \ldots, 32$.

Essa primeira abordagem ainda é incompleta, bastando analisar o número de graus de liberdade bosônicos e fermiônicos.

- bósons: as coordenadas do espaço alvo constituem 10 componentes reais. Devido à simetria de reparametrização no volume-mundo, é possível eliminar $p+1$ componentes. Desse modo, há $9-p$ graus de liberdade bosônicos.

- férmions: as coordenadas fermiônicas são restritas pelas condições de Majorana-Weyl. Isso implica que cada espinor contribui com 16 graus de liberdade. A simetria kapa e as equações de movimento reduzem esse número para 8.

Requerendo, portanto, supersimetria, há um déficit de $p-1$ graus de liberdade bosônicos.

Ao analisar a presença da $\mathrm{D} p$-brana no espaço-tempo, nota-se que a simetria de Lorentz $S O(1,9)$ é quebrada para $S O(1, p) \otimes S O(9-p)$. Pelo espectro não massivo da corda aberta, há um campo de calibre $A_{\alpha}$ sobre o volume-mundo da brana, pertencendo à representação vetorial de $S O(1, p)$. Tal campo possui $(p+1)$ componentes reais. A simetria de calibre e as equações de movimento reduzem esse número para $(p-1)$ graus de liberdade propagantes, precisamente o número necessário para completar a descrição da D-brana.

Ao incluir esse novo campo de calibre, é necessário investigar suas transformações de supersimetria e simetria kapa. A princípio, não há informação alguma sobre elas. Entretanto, há uma forma bastante consistente para implementar essa construção, como será visto a seguir.

\subsubsection{Supersimetria e Simetria kapa do Campo de Calibre}

O primeiro passo para determinar a transformação de supersimetria do campo de calibre $A_{\alpha}$ é identificar seus componentes. De forma geral, $\delta_{\xi} A_{\alpha}=F\left(X^{\mu}, \Theta^{\mathcal{A}}, \xi^{\mathcal{A}}\right)$, de tal modo que $F\left(X^{\mu}, \Theta^{\mathcal{A}}, \xi^{\mathcal{A}}\right)$ é linear no parâmetro $\xi^{\mathcal{A}}$ da transformação. A construção mais simples possível (a menos de transformações de calibre) é:

$$
\delta_{\xi} A_{\alpha}=\sum_{\mathcal{A}=1}^{2} c^{\mathcal{A}} \partial_{\alpha} X^{\mu}\left(\bar{\Theta}^{\mathcal{A}} \Gamma_{\mu} \xi^{\mathcal{A}}\right)+\sum_{\mathcal{A}, \mathcal{B}=1}^{2} d^{\mathcal{A B}}\left(\bar{\Theta}^{\mathcal{A}} \Gamma^{\mu} \xi^{\mathcal{A}}\right)\left(\bar{\Theta}^{\mathcal{B}} \Gamma_{\mu} \partial_{\alpha} \Theta^{\mathcal{B}}\right)
$$

Aqui a soma nos índices de supersimetria está explícita e isso será adotado no resto da subseção. $c^{\mathcal{A}} \mathrm{e}$ $d^{\mathcal{A B}}$ são coeficientes a serem determinados. Pela condição de realidade de $A_{\alpha}, c^{\mathcal{A}}$ deve ser puramente imaginário enquanto $d^{\mathcal{A B}}$ é real.

A determinação dos coeficientes acima é realizada pela imposição da álgebra de supersimetria. Para uma transformação pura (apenas em uma das supersimetrias) a comutação de duas transformações com parâmetros $\xi_{(1)}^{\mathcal{A}}$ e $\xi_{(2)}^{\mathcal{A}}$ é dada por:

$$
\begin{aligned}
{\left[\delta_{(1)}^{\mathcal{A}}, \delta_{(2)}^{\mathcal{A}}\right] A_{\alpha} } & =c^{\mathcal{A}}\left\{\delta_{(1)}^{\mathcal{A}}\left[\partial_{\alpha} X^{\mu}\left(\bar{\Theta}^{\mathcal{A}} \Gamma_{\mu} \xi_{(2)}^{\mathcal{A}}\right)\right]-\delta_{(2)}^{\mathcal{A}}\left[\partial_{\alpha} X^{\mu}\left(\bar{\Theta}^{\mathcal{A}} \Gamma_{\mu} \xi_{(1)}^{\mathcal{A}}\right)\right]\right\} \\
& +\sum_{\mathcal{B}=1}^{2} d^{\mathcal{A} \mathcal{B}}\left\{\delta_{(1)}^{\mathcal{A}}\left[\left(\bar{\Theta}^{\mathcal{A}} \Gamma^{\mu} \xi_{(2)}^{\mathcal{A}}\right)\left(\bar{\Theta}^{\mathcal{B}} \Gamma_{\mu} \partial_{\alpha} \Theta^{\mathcal{B}}\right)\right]-\delta_{(2)}^{\mathcal{A}}\left[\left(\bar{\Theta}^{\mathcal{A}} \Gamma^{\mu} \xi_{(1)}^{\mathcal{A}}\right)\left(\bar{\Theta}^{\mathcal{B}} \Gamma_{\mu} \partial_{\alpha} \Theta^{\mathcal{B}}\right)\right]\right\}
\end{aligned}
$$


Substituindo as relações dadas em (4.1) na transformação acima, obtem-se:

$$
\begin{aligned}
{\left[\delta_{(1)}^{\mathcal{A}}, \delta_{(2)}^{\mathcal{A}}\right] A_{\alpha} } & =c^{\mathcal{A}}\left\{i\left(\partial_{\alpha} \bar{\Theta}^{\mathcal{A}} \Gamma^{\mu} \xi_{(1)}^{\mathcal{A}}\right)\left(\bar{\Theta}^{\mathcal{A}} \Gamma_{\mu} \xi_{(2)}^{\mathcal{A}}\right)+\partial_{\alpha} X^{\mu}\left(\bar{\xi}_{(1)}^{\mathcal{A}} \Gamma_{\mu} \xi_{(2)}^{\mathcal{A}}\right)\right\} \\
& -c^{\mathcal{A}}\left\{i\left(\partial_{\alpha} \bar{\Theta}^{\mathcal{A}} \Gamma^{\mu} \xi_{(2)}^{\mathcal{A}}\right)\left(\bar{\Theta}^{\mathcal{A}} \Gamma_{\mu} \xi_{(1)}^{\mathcal{A}}\right)+\partial_{\alpha} X^{\mu}\left(\bar{\xi}_{(2)}^{\mathcal{A}} \Gamma_{\mu} \xi_{(1)}^{\mathcal{A}}\right)\right\} \\
& +d^{\mathcal{A} \mathcal{A}}\left\{\left(\bar{\xi}_{(1)}^{\mathcal{A}} \Gamma^{\mu} \xi_{(2)}^{\mathcal{A}}\right)\left(\bar{\Theta}^{\mathcal{A}} \Gamma_{\mu} \partial_{\alpha} \Theta^{\mathcal{A}}\right)+\left(\bar{\Theta}^{\mathcal{A}} \Gamma^{\mu} \xi_{(2)}^{\mathcal{A}}\right)\left(\bar{\xi}_{(1)}^{\mathcal{A}} \Gamma_{\mu} \partial_{\alpha} \Theta^{\mathcal{A}}\right)\right\} \\
& -d^{\mathcal{A} \mathcal{A}}\left\{\left(\bar{\xi}_{(2)}^{\mathcal{A}} \Gamma^{\mu} \xi_{(1)}^{\mathcal{A}}\right)\left(\bar{\Theta}^{\mathcal{A}} \Gamma_{\mu} \partial_{\alpha} \Theta^{\mathcal{A}}\right)+\left(\bar{\Theta}^{\mathcal{A}} \Gamma^{\mu} \xi_{(1)}^{\mathcal{A}}\right)\left(\bar{\xi}_{(2)}^{\mathcal{A}} \Gamma_{\mu} \partial_{\alpha} \Theta^{\mathcal{A}}\right)\right\} \\
& +d_{\mathcal{B} \neq \mathcal{A}}^{\mathcal{A B}}\left\{2\left(\bar{\Theta}^{\mathcal{B}} \Gamma^{\mu} \partial_{\alpha} \Theta^{\mathcal{B}}\right)\left(\bar{\xi}_{(1)}^{\mathcal{A}} \Gamma_{\mu} \xi_{(2)}^{\mathcal{A}}\right)\right\} .
\end{aligned}
$$

A propriedade (B.4) é recorrente nessa demonstração. Rearranjando os termos:

$$
\begin{aligned}
{\left[\delta_{(1)}^{\mathcal{A}}, \delta_{(2)}^{\mathcal{A}}\right] A_{\alpha} } & =2 d^{\mathcal{A} \mathcal{A}}\left(\bar{\xi}_{(1)}^{\mathcal{A}} \Gamma^{\mu} \xi_{(2)}^{\mathcal{A}}\right)\left(\bar{\Theta}^{\mathcal{A}} \Gamma_{\mu} \partial_{\alpha} \Theta^{\mathcal{A}}\right) \\
& +\left(^{\mathcal{A} \mathcal{A}}-i c^{\mathcal{A}}\right)\left[\left(\bar{\Theta}^{\mathcal{A}} \Gamma^{\mu} \xi_{(2)}^{\mathcal{A}}\right)\left(\bar{\xi}_{(1)}^{\mathcal{A}} \Gamma_{\mu} \partial_{\alpha} \Theta^{\mathcal{A}}\right)-(1 \leftrightarrow 2)\right] \\
& +\underset{\mathcal{B} \neq \mathcal{A}}{d_{\mathcal{A}}^{\mathcal{A B}}}\left\{2\left(\bar{\Theta}^{\mathcal{B}} \Gamma^{\mu} \partial_{\alpha} \Theta^{\mathcal{B}}\right)\left(\bar{\xi}_{(1)}^{\mathcal{A}} \Gamma_{\mu} \xi_{(2)}^{\mathcal{A}}\right)\right\}+2 \partial_{\alpha}\left[X^{\mu}\left(\bar{\xi}_{(1)}^{\mathcal{A}} \Gamma_{\mu} \xi_{(2)}^{\mathcal{A}}\right)\right]
\end{aligned}
$$

A álgebra de supersimetria é requerida a menos de transformações de calibre e o último termo na igualdade acima representa justamente isso. Os outros termos claramente não estão associados a uma translação do campo de calibre. Dessa forma, os coeficientes $d^{12}$ e $d^{21}$ são obrigatoriamente nulos. Pela propriedade (B.6) do apêndice,

$$
\left(\bar{\xi}_{(1)}^{\mathcal{A}} \Gamma^{\mu} \xi_{(2)}^{\mathcal{A}}\right)\left(\bar{\Theta}^{\mathcal{A}} \Gamma_{\mu} \partial_{\alpha} \Theta^{\mathcal{A}}\right)=\left(\bar{\Theta}^{\mathcal{A}} \Gamma^{\mu} \xi_{(2)}^{\mathcal{A}}\right)\left(\bar{\xi}_{(1)}^{\mathcal{A}} \Gamma_{\mu} \partial_{\alpha} \Theta^{\mathcal{A}}\right)-\left(\bar{\Theta}^{\mathcal{A}} \Gamma^{\mu} \xi_{(1)}^{\mathcal{A}}\right)\left(\bar{\xi}_{(2)}^{\mathcal{A}} \Gamma_{\mu} \partial_{\alpha} \Theta^{\mathcal{A}}\right)
$$

e a relação de comutação pode ser reescrita como

$$
\left[\delta_{(1)}^{\mathcal{A}}, \delta_{(2)}^{\mathcal{A}}\right] A_{\alpha} \sim\left(3 d^{\mathcal{A} \mathcal{A}}-i c^{\mathcal{A}}\right)\left(\bar{\Theta}^{\mathcal{A}} \Gamma^{\mu} \xi_{(2)}^{\mathcal{A}}\right)\left(\bar{\xi}_{(1)}^{\mathcal{A}} \Gamma_{\mu} \partial_{\alpha} \Theta^{\mathcal{A}}\right)-(1 \leftrightarrow 2) .
$$

Nessa expressão, o símbolo " " representa a igualdade a menos de transformações de calibre. A única forma de anular a expressão é tomar $3 d^{\mathcal{A} \mathcal{A}}=i c^{\mathcal{A}}$, restringindo fortemente a transformação (5.5):

$$
\delta_{\xi} A_{\alpha}=\sum_{\mathcal{A}=1}^{2} c^{\mathcal{A}}\left[\partial_{\alpha} X^{\mu}\left(\bar{\Theta}^{\mathcal{A}} \Gamma_{\mu} \xi^{\mathcal{A}}\right)+\frac{i}{3}\left(\bar{\Theta}^{\mathcal{A}} \Gamma^{\mu} \xi^{\mathcal{A}}\right)\left(\bar{\Theta}^{\mathcal{A}} \Gamma_{\mu} \partial_{\alpha} \Theta^{\mathcal{A}}\right)\right]
$$

Analisando agora as transformações de supersimetria mistas, com parâmetros $\xi^{1}$ e $\xi^{2}$ :

$$
\left[\delta^{1}, \delta^{2}\right] A_{\alpha}=c^{2} \delta^{1}\left[\partial_{\alpha} X^{\mu}\left(\bar{\Theta}^{2} \Gamma_{\mu} \xi^{2}\right)\right]-c^{1} \delta^{2}\left[\partial_{\alpha} X^{\mu}\left(\bar{\Theta}^{1} \Gamma_{\mu} \xi^{1}\right)\right]
$$

Apenas o primeiro termo em (5.6) contribui, pois $\delta^{1} \Theta^{2}=\delta^{2} \Theta^{1}=0$. Prosseguindo:

$$
\left[\delta^{1}, \delta^{2}\right] A_{\alpha}=c^{1}\left(\bar{\xi}^{2} \Gamma^{\mu} \partial_{\alpha} \Theta^{2}\right)\left(\bar{\Theta}^{1} \Gamma_{\mu} \xi^{1}\right)-c^{2}\left(\bar{\xi}^{1} \Gamma^{\mu} \partial_{\alpha} \Theta^{1}\right)\left(\bar{\Theta}^{2} \Gamma_{\mu} \xi^{2}\right) .
$$

A menos de transformações de calibre,

$$
\left[\delta^{1}, \delta^{2}\right] A_{\alpha} \sim\left(c^{1}+c^{2}\right)\left(\bar{\xi}^{2} \Gamma^{\mu} \partial_{\alpha} \Theta^{2}\right)\left(\bar{\Theta}^{1} \Gamma_{\mu} \xi^{1}\right),
$$

concluindo que $c^{1}=-c^{2}$. 
Portanto, ao requerer a álgebra de supersimetria, obtem-se a lei de transformação do campo de calibre:

$$
\delta_{\xi} A_{\alpha} \propto 3 i\left(\partial_{\alpha} X^{\mu}\right)\left(\bar{\Theta}^{1} \Gamma_{\mu} \xi^{1}-\bar{\Theta}^{2} \Gamma_{\mu} \xi^{2}\right)-\left(\bar{\Theta}^{1} \Gamma^{\mu} \xi^{1}\right)\left(\bar{\Theta}^{1} \Gamma_{\mu} \partial_{\alpha} \Theta^{1}\right)+\left(\bar{\Theta}^{2} \Gamma^{\mu} \xi^{2}\right)\left(\bar{\Theta}^{2} \Gamma_{\mu} \partial_{\alpha} \Theta^{2}\right) .
$$

Estendendo a análise ao tensor do campo, $F_{\alpha \beta}=\partial_{\alpha} A_{\beta}-\partial_{\beta} A_{\alpha}$, obtem-se:

$$
\begin{aligned}
\delta_{\xi} F_{\alpha \beta} & \propto i\left(\partial_{\alpha} X^{\mu}\right) \partial_{\beta}\left(\bar{\Theta}^{1} \Gamma_{\mu} \xi^{1}-\bar{\Theta}^{2} \Gamma_{\mu} \xi^{2}\right)-i\left(\partial_{\beta} X^{\mu}\right) \partial_{\alpha}\left(\bar{\Theta}^{1} \Gamma_{\mu} \xi^{1}-\bar{\Theta}^{2} \Gamma_{\mu} \xi^{2}\right) \\
& +\left(\bar{\Theta}^{1} \Gamma^{\mu} \xi^{1}\right)\left(\partial_{\alpha} \bar{\Theta}^{1} \Gamma_{\mu} \partial_{\beta} \Theta^{1}\right)-\left(\bar{\Theta}^{2} \Gamma^{\mu} \xi^{12}\right)\left(\partial_{\alpha} \bar{\Theta}^{2} \Gamma_{\mu} \partial_{\beta} \Theta^{2}\right) .
\end{aligned}
$$

Na linguagem de formas diferenciais, reescreve-se a transformação como

$$
\delta_{\xi} F \propto 2 i\left(d X^{\mu}\right)\left(d \bar{\Theta}^{1} \Gamma_{\mu} \xi^{1}-d \bar{\Theta}^{2} \Gamma_{\mu} \xi^{2}\right)+\left(\bar{\Theta}^{1} \Gamma^{\mu} \xi^{1}\right)\left(d \bar{\Theta}^{1} \Gamma_{\mu} d \Theta^{1}\right)-\left(\bar{\Theta}^{2} \Gamma^{\mu} \xi^{12}\right)\left(d \bar{\Theta}^{2} \Gamma_{\mu} d \Theta^{2}\right) .
$$

A lei de transformação de $F_{\alpha \beta}$ lembra bastante a transformação de supersimetria da 2-forma (4.4), construída sobre a folha-mundo para introduzir a simetria kapa no formalismo GS. Generalizando para uma dimensão arbitrária de volume-mundo, sua transformação de supersimetria é dada por:

$$
\begin{aligned}
\delta_{\xi} \Omega_{2} & \propto i\left(d X^{\mu}\right)\left(d \bar{\Theta}^{1} \Gamma_{\mu} \xi^{1}-d \bar{\Theta}^{2} \Gamma_{\mu} \xi^{2}\right)+\sum_{\mathcal{A}=1}^{2}\left(d \bar{\Theta}^{\mathcal{A}} \Gamma_{\mu} \xi^{\mathcal{A}}\right)\left(\bar{\Theta}^{1} \Gamma^{\mu} d \Theta^{1}-\bar{\Theta}^{2} \Gamma^{\mu} d \Theta^{2}\right) \\
& +\left\{\left(d \bar{\Theta}^{1} \Gamma_{\mu} \xi^{1}\right)\left(\bar{\Theta}^{2} \Gamma_{\mu} d \Theta^{2}\right)+\left(\bar{\Theta}^{1} \Gamma^{\mu} d \Theta^{1}\right)\left(d \bar{\Theta}^{2} \Gamma_{\mu} \xi^{2}\right)\right\} .
\end{aligned}
$$

Após algumas simplificações:

$$
\delta_{\xi} \Omega_{2} \propto i\left(d X^{\mu}\right)\left(d \bar{\Theta}^{1} \Gamma_{\mu} \xi^{1}-d \bar{\Theta}^{2} \Gamma_{\mu} \xi^{2}\right)+\left(d \bar{\Theta}^{1} \Gamma_{\mu} \xi^{1}\right)\left(\bar{\Theta}^{1} \Gamma^{\mu} d \Theta^{1}\right)-\left(d \bar{\Theta}^{2} \Gamma_{\mu} \xi^{2}\right)\left(\bar{\Theta}^{2} \Gamma^{\mu} d \Theta^{2}\right) .
$$

Pelas propriedades discutidas no final do apêndice B, obtem-se

$$
\delta_{\xi} \Omega_{2} \propto i\left(d X^{\mu}\right)\left(d \bar{\Theta}^{1} \Gamma_{\mu} \xi^{1}-d \bar{\Theta}^{2} \Gamma_{\mu} \xi^{2}\right)+\frac{1}{2}\left(\bar{\Theta}^{1} \Gamma^{\mu} \xi^{1}\right)\left(d \bar{\Theta}^{1} \Gamma_{\mu} d \Theta^{1}\right)-\frac{1}{2}\left(\bar{\Theta}^{2} \Gamma^{\mu} \xi^{12}\right)\left(d \bar{\Theta}^{2} \Gamma_{\mu} d \Theta^{2}\right),
$$

precisamente a lei de transformação de $F_{\alpha \beta}$. Como toda a análise é feita a menos de constantes de proporcionalidade, define-se

$$
\delta_{\xi} A=i\left(d X^{\mu}\right)\left(\bar{\Theta}^{1} \Gamma_{\mu} \xi^{1}-\bar{\Theta}^{2} \Gamma_{\mu} \xi^{2}\right)-\frac{1}{3}\left(\bar{\Theta}^{1} \Gamma^{\mu} \xi^{1}\right)\left(\bar{\Theta}^{1} \Gamma_{\mu} d \Theta^{1}\right)+\frac{1}{3}\left(\bar{\Theta}^{2} \Gamma^{\mu} \xi^{2}\right)\left(\bar{\Theta}^{2} \Gamma_{\mu} d \Theta^{2}\right) .
$$

Assim, é construído o objeto supersimétrico

$$
\mathcal{F}_{\alpha \beta}=F_{\alpha \beta}+b_{\alpha \beta},
$$

em que

$$
b=i\left(d X^{\mu}\right)\left(\bar{\Theta}^{1} \Gamma_{\mu} d \Theta^{1}-\bar{\Theta}^{2} \Gamma_{\mu} d \Theta^{2}\right)+\left(\bar{\Theta}^{1} \Gamma^{\mu} d \Theta^{1}\right)\left(\bar{\Theta}^{2} \Gamma_{\mu} d \Theta^{2}\right) .
$$

No caso da simetria kapa, o procedimento é análogo. Analisando o comportamento dos campos sob essas transformações, surge uma observação interessante: simetria kapa e supersimetria comutam. Desse modo, é natural investigar a lei de transformação de simetria kapa do campo de calibre requerendo a comutação com a supersimetria.

Uma construção similar àquela em (5.5) é um bom ponto de partida. Levando em conta que o parâmetro da simetria kapa é local, uma generalização é necessária, afinal surgem termos não equivalentes por transformações de calibre:

$$
\begin{aligned}
\delta_{\kappa} A_{\alpha} & =\sum_{\mathcal{A}=1}^{2} C^{\mathcal{A}} \Pi_{\alpha}^{\mu}\left[\bar{\Theta}^{\mathcal{A}} \Gamma_{\mu}\left(\delta_{\kappa} \Theta^{\mathcal{A}}\right)\right]+D^{\mathcal{A}} X^{\mu}\left[\partial_{\alpha} \bar{\Theta}^{\mathcal{A}} \Gamma_{\mu}\left(\delta_{\kappa} \Theta^{\mathcal{A}}\right)\right] \\
& +\sum_{\mathcal{A}, \mathcal{B}=1}^{2} E^{\mathcal{A} \mathcal{B}}\left[\bar{\Theta}^{\mathcal{A}} \Gamma^{\mu}\left(\delta_{\kappa} \Theta^{\mathcal{A}}\right)\right]\left(\bar{\Theta}^{\mathcal{B}} \Gamma_{\mu} \partial_{\alpha} \Theta^{\mathcal{B}}\right) .
\end{aligned}
$$


Ao requerer a comutação com a supersimetria, os coeficientes $C^{\mathcal{A}}, D^{\mathcal{A}}$ e $E^{\mathcal{A B}}$ são fixados e o resultado, na notação de formas diferenciais, é dado por:

$$
\begin{aligned}
\delta_{\kappa} A & =i \Pi^{\mu}\left[\left(\delta_{\kappa} \bar{\Theta}^{1}\right) \Gamma_{\mu} \Theta^{1}-\left(\delta_{\kappa} \bar{\Theta}^{2}\right) \Gamma_{\mu} \Theta^{2}\right] \\
& +\left(\bar{\Theta}^{1} \Gamma_{\mu} d \Theta^{1}\right)\left[\bar{\Theta}^{2} \Gamma^{\mu}\left(\delta_{\kappa} \Theta^{2}\right)\right]-\left(\bar{\Theta}^{2} \Gamma_{\mu} d \Theta^{2}\right)\left[\bar{\Theta}^{1} \Gamma^{\mu}\left(\delta_{\kappa} \Theta^{1}\right)\right] .
\end{aligned}
$$

Portanto, as leis de transformação por simetria kapa e supersimetria foram obtidas requerendo a algebra de supersimetria e a comutação entre simetrias diferentes. O critério de simplicidade utilizado em $[15,16]$ leva à transformação acima, necessária para a construção da ação efetiva, discutida a seguir.

\subsubsection{Ação Efetiva}

A maneira mais natural de construir a ação efetiva das D-branas é partir de uma ação de volumemundo, como foi visto para as p-branas. Há dois requisitos para uma possível generalização. O primeiro, e mais simples de ser implementado, é a supersimetria. Para isso, há à disposição os objetos definidos em (4.2) e(5.8). Definindo

$$
G_{\alpha \beta}=\Pi_{\alpha}^{\mu} \Pi_{\beta}^{\nu} \eta_{\mu \nu}
$$

é construída a seguinte ação de volume-mundo supersimétrica:

$$
S_{\mathrm{D} p}^{\prime}=-T_{\mathrm{D} p} \int d^{p+1} \sigma \sqrt{-\operatorname{det}\left(G_{\alpha \beta}+k \mathcal{F}_{\alpha \beta}\right)}
$$

Aqui, $T_{\mathrm{D} p}$ é a tensão da $\mathrm{D} p$-brana e $k$ é uma constante escrita em função dos parâmetros da teoria. Sua determinação é bastante simples. Na seção 2.8 foi analisada a linha de Wilson e a questão da dualidade $\mathrm{T}$ nas D-branas e verificou-se a correspondência entre as coordenadas de uma dimensão compactificada e as componentes de um campo de calibre. Ao verificar essa correspondência precisamente, mostra-se que $k=\pi l_{s}^{2}$.

A ação acima definida não cumpre o segundo requisito, a simetria kapa. Sua implementação é similar àquela do capítulo anterior. Nesse caso, o volume-mundo é $(p+1)$ dimensional e o termo que assegura a simetria kapa é obtido pela construção de um espaço de dimensão $(p+2)$ cuja fronteira é o volume-mundo.

$$
S_{\mathrm{D} p}^{\prime \prime}=\int_{\mathcal{M}} \Omega_{p+2}=\int_{\partial \mathcal{M}} \Omega_{p+1} \quad \operatorname{com} \Omega_{p+2}=d \Omega_{p+1} .
$$

Novamente, para construir uma forma $\Omega_{p+2}$ supersimétrica, dispõe-se de $d \Theta^{1}, d \Theta^{2}, \Pi^{\mu}$ e $\mathcal{F}$. De forma geral,

$$
\Omega_{p+2}=d \bar{\Theta}^{\mathcal{A}} \mathcal{T}_{p}^{\mathcal{A B}} d \Theta^{\mathcal{B}}
$$

em que $\mathcal{T}_{p}$ é uma matriz $2 \times 2$ cujos elementos são $p$-formas compostas pelo produto de até $p$ matrizes de Dirac $\Gamma^{\mu}$.

A partir de uma análise cuidadosa é possível impor algumas restrições sobre a forma matricial de $\mathcal{T}_{p}$. Reescrevendo as propriedades (B.4) e (B.5) na forma acima proposta, obtem-se:

- $d \bar{\Theta}^{\mathcal{A}} \Gamma^{\mu_{1} \ldots \mu_{p}} d \Theta^{\mathcal{B}}=-(-1)^{\frac{p(p+1)}{2}} d \bar{\Theta}^{\mathcal{B}} \Gamma^{\mu_{1} \ldots \mu_{p}} d \Theta^{\mathcal{A}}$.

- $d \bar{\Theta}^{\mathcal{A}} \Gamma^{\mu_{1} \ldots \mu_{p}} d \Theta^{\mathcal{B}}=(-1)^{p+1} \epsilon^{\mathcal{A}} \epsilon^{\mathcal{B}} d \bar{\Theta}^{\mathcal{A}} \Gamma^{\mu_{1} \ldots \mu_{p}} d \Theta^{\mathcal{B}}$. Aqui os índices repetidos não indicam soma e $\epsilon^{\mathcal{A}}$ representa a quiralidade dos espinores, tal que $\Gamma^{D} \Theta^{\mathcal{A}}=\epsilon^{\mathcal{A}} \Theta^{\mathcal{A}}$.

A segunda condição está diretamente associada à estabilidade das $\mathrm{D} p$-branas nas teorias $I I$-A e $I I$-B. Como foi visto, os campos de Ramond-Ramond, presentes no espectro das cordas fechadas, acoplam-se 
com as branas da forma descrita na seção 5.1. É possível mostrar que as D p-branas são estáveis na teoria $I I$-A quando $p$ é par e, quando $p$ é ímpar, apenas a teoria $I I$-B apresenta estabilidade.

Nota-se, pelas propriedades descritas, que:

$$
\begin{aligned}
\mathcal{T}_{p}^{I I-\mathrm{A}} & \approx\left(\begin{array}{cc}
0 \text {-forma } \\
0 & 1 \\
-1 & 0
\end{array}\right)+\left(\begin{array}{cc}
2 \text {-forma } \\
D_{2}^{1} & C_{2} \\
C_{2} & D_{2}^{2}
\end{array}\right)+\left(\begin{array}{cc}
4 \text {-forma } \\
0 & C_{4} \\
-C_{4} & 0
\end{array}\right)+\ldots \\
\mathcal{T}_{p}^{I I-\mathrm{B}} & \approx\left(\begin{array}{cc}
D_{1}^{1} & C_{1} \\
C_{1} & D_{1}^{2} \\
1 \text {-forma }
\end{array}\right)+\left(\begin{array}{cc}
0 & C_{3} \\
-C_{3} & 0 \\
3 \text {-forma }
\end{array}\right)+\left(\begin{array}{cc}
D_{5}^{1} & C_{5} \\
C_{5} & D_{5}^{2} \\
5 \text {-forma }
\end{array}\right)+\ldots
\end{aligned}
$$

Aqui, $D_{n}^{1,2}$ e $C_{n}$ representam simplesmente as componentes que não se anulam pelas propriedades (B.4) e (B.5). Uma dedução rigorosa pode ser encontrada em [16] e leva ao seguinte resultado:

$$
\mathcal{T}^{\mathcal{A B}}=\sum_{p=0}^{p \leq 10} \mathcal{T}_{p}^{\mathcal{A B}}=T_{\mathrm{D} 0} e^{k \mathcal{F}} f(\Psi) .
$$

Aqui, $f(\Psi)$ é uma matriz $2 \times 2, \operatorname{com} \Psi=\frac{1}{\sqrt{k}} \Gamma_{\mu} \Pi^{\mu}$, tal que:

$$
\begin{aligned}
f^{I I-\mathrm{A}} & =\left(\begin{array}{cc}
0 & \cos \Psi \\
-\cosh \Psi & 0
\end{array}\right) \\
f^{I I-\mathrm{B}} & =\left(\begin{array}{cc}
0 & \sin \Psi \\
\sinh \Psi & 0
\end{array}\right) .
\end{aligned}
$$

É importante lembrar que $\Psi$ é uma 1-forma, de modo que $f(\Psi)$ e $e^{k \mathcal{F}}$ correspondem a somatórios de formas diferenciais de grau diferente. Para obter o termo associado a uma D $p$-brana basta analisar as combinações possíveis que geram $p$-formas.

A construção do termo geral para a implementação da simetria kapa baseia-se no fato de que existe uma relação entre as tensões de duas D-branas diferentes. Com base na expansão de (5.10) verifica-se, pelo termo cinético do campo de calibre $F_{\alpha \beta} F^{\alpha \beta}$, que a tensão da $\mathrm{D} p$-brana deve ter a forma $c_{p} / g_{s}$, em que $c_{p}$ é um fator numérico e $g_{s}$ é a constante de acoplamento da corda fechada.

Como uma $\mathrm{D} p$-brana envolvendo uma dimensão compactificada pode ser vista, através de uma dualidade $\mathrm{T}$, como uma $\mathrm{D}(p-1)$-brana, estabelece-se que

$$
2 \pi R T_{\mathrm{D} p}=T_{\mathrm{D} p-1} \Rightarrow 2 \pi R \frac{c_{p}}{g_{s}}=\frac{c_{p-1}}{\tilde{g}_{s}}
$$

de acordo com a subseção 4.7.3. Portanto, pela equação (4.27), obtem-se uma relação de recorrência que permite determinar a tensão de todas as D-branas em função da tensão da D0-brana e do comprimento da corda:

$$
T_{\mathrm{D} p}=T_{\mathrm{D} 0}\left(\frac{1}{\pi l_{s} \sqrt{2}}\right)^{p}
$$

Isso encerra a introdução à dinâmica das D-branas, cuja ação efetiva no limite de baixas energias,

$$
S_{\mathrm{D} p}=-T_{\mathrm{D} p} \int d^{p+1} \sigma \sqrt{-\operatorname{det}\left(G_{\alpha \beta}+k \mathcal{F}_{\alpha \beta}\right)}+\int \Omega_{p+1}
$$

possui supersimetria e simetria kapa por construção.

$\mathrm{Na}$ próxima seção são introduzidas as ações efetivas de supergravidade também no limite de baixas energias. 


\subsection{Ações da Supergravidade}

A supergravidade é uma generalização da Relatividade Geral com a inclusão de campos fermiônicos, introduzindo a supersimetria localmente. Geralmente é reproduzida através de ações efetivas de limites de baixas energias de teorias mais fundamentais, como a teoria de supercordas e a teoria M.

\subsubsection{Teoria $\mathbf{M}$ e Supergravidade $D=11$}

Ao estudar a dinâmica das cordas, verificou-se que existem, de fato, 5 tipos diferentes de teorias: tipo $I$, tipos $I I$-A e $I I$-B e as cordas heteróticas $S O(32)$ e $E_{8} \times E_{8}$. Os três primeiros tipos foram descritos introdutoriamente na dissertação, incluindo a apresentação do seu conteúdo não massivo. As cordas heteróticas não foram mencionadas pois sua construção é um pouco mais sutil. O nome heterótica tem origem na forma como a teoria é construída. Ao analisar as soluções das coordenadas do espaço-alvo, verificou-se que esta podia ser dividida campos que se propagam em direções opostas na folha-mundo, denominadas genericamente de direita e esquerda. A corda heterótica assume que os campos que se propagam para a direita são os mesmos da corda bosônica, vivendo, inclusive, em $D=26$. Os campos que se propagam para a esquerda incluem a construção supersimétrica e vivem em $D=10$. Por incrível que possa parecer, as cordas heteróticas constituem uma teoria consistente do ponto de vista físico e acreditou-se, durante muito tempo, que eram as teorias com maior potencial de descrição da natureza.

Ao aprofundar os estudos sobre os cinco tipos de teorias de supercordas, gradualmente foram descobertas uma série de dualidades que poderiam relacionar cada uma das teorias entre si. Uma dessas dualidade foi descrita nos capítulos 2 e 4, a dualidade $\mathrm{T}$, que relaciona as supercordas $I I$-A e $I I$-B. Há ainda a dualidade $\mathrm{S}$, que relaciona os regimes de acoplamento forte de uma teoria com o acoplamento fraco de outra; a dualidade U, que é uma combinação das dualidades T e S; a simetria espelho e as transições de conifold. Os detalhes são muitos, mas pouco relevantes para o nível aqui descrito. O ponto importante é que essa ligação entre todas as cinco teorias sugere uma unificação. A proposta foi realizada na chamada segunda revolução da teoria de cordas, em que conjecturou-se a existência da teoria M, que dá origem, pelas dualidades citadas, à todas as teorias de supercorda conhecidas.

Muitos avanços foram alcançados, mas até hoje não existe uma descrição completa da teoria M. Sua característica mais marcante é que existe em um espaço-tempo de 11 dimensões. Conhecido é, entretanto, seu regime de baixas energias, que dá origem a supergravidade $D=11$ [17]. Esta subseção descreve seu conteúdo de campos e sua ação efetiva.

No limite de baixas energias as representações de estados de uma partícula pertencem ao grupo $S O(D-2)$ e esse fato é um bom guia para a composição dos campos.

Naturalmente, a teoria deve incluir o gráviton. Como foi visto anteriormente, o gráviton está associado a um tensor de segunda ordem simétrico e de traço nulo do grupo $S O(D-2)$. Desse modo, possui

$$
\frac{1}{2}(D-1)(D-2)-1
$$

graus de liberdade, o que em $D=11$ dá um total de 44 . O gráviton é representado pelo tensor métrico $G^{M N}, \operatorname{com} M, N=0, \ldots, 10$.

O conteúdo bosônico ainda não está completo, pela discussão em C.3.2, restam ainda 84 graus de liberdade a serem "preenchidos". Uma 3-forma $A_{3}$ fornece precisamente esse valor. Pela simetria de calibre, $A_{3} \rightarrow A_{3}+d \Lambda_{2}$, apenas as componentes transversais se propagam, de modo que um tensor de terceira ordem totalmente antissimétrico de $S O(9)$ possui

$$
\left(\begin{array}{l}
9 \\
3
\end{array}\right)=\frac{9 !}{3 ! 6 !}=84
$$


graus de liberdade. Há uma forma de confirmar a necessidade da 3-forma. Por uma redução dimensional, $G^{M N}$ e $A^{M N P}$ reproduzem o conteúdo bosônico dos espectro não massivo da supercorda $I I$-A. Os termos cinéticos dos campos bosônicos não apresentam nenhuma particularidade:

$$
S \sim \int d^{11} x \sqrt{-G}\left[R-\frac{1}{2}\left|F_{4}\right|^{2}\right] .
$$

Aqui, $G$ é o determinante da métrica, $R$ é o escalar de curvatura em 11 dimensões e $F_{4}=d A_{3}$, tal que $\left|F_{4}\right|^{2}=F_{\mu \nu \rho \sigma} F^{\mu \nu \rho \sigma}$.

O conteúdo fermiônico deve incluir um espinor de Rarita-Schwinger $\Psi_{A}^{M}$. Em $D=11$, a representação espinorial mínima possui 32 componentes. Impondo a condição de realidade, há 32 componentes reais. Como foi discutido em 3.1, a introdução de espinores no espaço-tempo requer o formalismo das vielbein, $E_{m}^{M}$, que permite a construção da teoria com simetria de Lorentz local. Aqui, $m$ é o índice do espaço tangente. O termo cinético de $\Psi_{A}^{M}$ é

$$
S \sim \int d^{11} x \sqrt{-G} \bar{\Psi}_{M} \Gamma^{M N P} D_{N} \Psi_{P}
$$

$D_{M}$ é a derivada covariante e $\Gamma^{M}=\Gamma^{m} E_{m}^{M}$.

Os espinores de Rarita-Schwinger devem pertencer a uma representação do grupo de cobertura $\operatorname{Spin}(9)$, possuindo 16 componentes espinoriais reais e 9 componentes vetoriais das direções transversais. Assim, há

$$
9 \cdot 16=128+16
$$

componentes. A ação possui a simetria $\delta \Psi_{M}=D_{M} \epsilon$, sendo $\epsilon$ um espinor qualquer. Desse modo, é possível eliminar 16 graus de liberdade e o espinor de Rarita-Schwinger contribui efetivamente com 128.

As transformações de supersimetria dos campos presentes na ação são

$$
\begin{aligned}
\delta E_{m}^{M} & =\bar{\epsilon} \Gamma_{m} \Psi^{M} \\
\delta A^{M N P} & =-3 \bar{\epsilon} \Gamma^{[M N} \Psi^{P]} \\
\delta \Psi_{M} & =D_{M} \epsilon+\frac{1}{12}\left(\Gamma_{M} F^{(4)}-3 F_{M}^{(4)}\right) \epsilon+\left(\Psi^{2}\right) \epsilon
\end{aligned}
$$

Aqui, a última relação é dada a menos de termos de ordem superior em $\Psi_{M} \mathrm{e}$

$$
\begin{aligned}
F^{(4)} & =\frac{1}{4 !} F_{M N P Q} \Gamma^{M N P Q}, \\
F_{M}^{(4)} & =\frac{1}{3 !} F_{M N P Q} \Gamma^{N P Q} .
\end{aligned}
$$

Como o objetivo principal do texto é o estudo das soluções clássicas, o campo fermiônico deve ser nulo. Entretanto, uma transformação de supersimetria pode violar tal condição, a menos que seu parâmetro tenha uma forma bem definida, isto é, para uma dada solução clássica é necessário encontrar os espinores de Killing $\epsilon$ da transformação, tal que

$$
D_{M} \epsilon+\frac{1}{12}\left(\Gamma_{M} F^{(4)}-3 F_{M}^{(4)}\right) \epsilon=0
$$

Se $\epsilon$ satisfaz à equação acima, então as trasnformações de supersimetria mantém o $\Psi_{M}=0$.

Como foi visto no começo do capítulo, a existência de $A_{3}$ sugere o acoplamento com branas, nesse caso M-branas. Uma 3-forma acopla-se eletricamente a uma 2-brana (ou M2-brana) e magneticamente a uma 5-brana (ou M5-brana). O interesse nas branas reside no fato de que são estados BPS saturados, o que as torna estáveis mesmo a altas energias. Ou seja, seu comportamento a baixas energias pode ser 
estudado e o resultado estendido a escalas não perturbativas. Uma discussão rápida sobre a estabilidade de estados BPS pode ser encontrada em [6].

A parte bosônica da supergravidade em $D=11$ é dada por:

$$
S_{11}=\frac{1}{2 \kappa_{11}^{2}}\left\{\int d^{11} x\left[R-\frac{1}{2}\left|F_{4}\right|^{2}\right]-\frac{1}{6} \int A_{3} \wedge F_{4} \wedge F_{4}\right\} .
$$

$\kappa_{11}$ é a constante de acoplamento gravitacional e está associado à constante gravitacional $G_{11}$ por

$$
16 \pi G_{11}=2 \kappa_{11}^{2}=\frac{1}{2 \pi}\left(2 \pi l_{P}\right)^{9}
$$

em que $l_{P}$ é o comprimento de Planck ${ }^{1}$ em $D=11$. Sendo $l_{P}$ a única escala na supergravidade $D=11$, as tensões das M-branas podem ser obtidas por uma simples análise dimensional:

$$
T_{M 2}=2 \pi\left(2 \pi l_{P}\right)^{-3}, \quad T_{M 5}=2 \pi\left(2 \pi l_{P}\right)^{-6} .
$$

A seguir, é introduzida a supergravidade associada à teoria $I I-\mathrm{A}$.

\subsubsection{Supergravidade $I I$-A}

A supergravidade $I I$-A pode ser obtida do limite de baixas energias da teoria M por uma redução dimensional. A redução dimensional pode ser vista como uma compactificação em que o raio da dimensão compactificada vai a zero. Em outras palavras, qualquer depedência em relação a essa coordenada é destruída, equivalentemente a tomar apenas o modo zero em uma expansão de Fourier.

O setor bosônico do espectro não massivo da teoria de supercordas $I I$-A é composto por

$$
\underbrace{\phi, g_{\mu \nu}, B_{\mu \nu}}_{N S-N S}, \quad \underbrace{A_{\mu}, A_{\mu \nu \rho}}_{R-R} .
$$

Aqui, $\mu=0, \ldots, 9$. Verifica-se que a redução dimensional dá origem a todos os campos acima:

$$
\begin{aligned}
G_{M N} & =e^{-\frac{2 \phi}{3}}\left(\begin{array}{cc}
g_{\mu \nu}+e^{2 \phi} A_{\mu} A_{\nu} & e^{2 \phi} A_{\mu} \\
e^{2 \phi} A_{\nu} & e^{2 \phi}
\end{array}\right), \\
A_{M N P} & \rightarrow\left\{\begin{array}{l}
A_{\mu \nu 10} \equiv B_{\mu \nu} \\
A_{\mu \nu \rho} .
\end{array}\right.
\end{aligned}
$$

A disposição do campo do dílaton é escolhida de forma conveniente para a construção da ação, que será introduzida adiante.

O setor fermiônico possui representações não massivas de $\left(\boldsymbol{8}_{v}+\boldsymbol{8}_{s}\right)$ e $\left(\boldsymbol{8}_{v}+\boldsymbol{8}_{c}\right)$, representadas por dois gravitinos e dois dilatinos, os superparceiros de gráviton e do dílaton, respectivamente. A redução dimensional fornece:

$$
\Psi_{M} \rightarrow\left\{\begin{array}{l}
\Psi_{10} \equiv \Phi_{ \pm} \\
\Psi_{\mu \pm}
\end{array}\right.
$$

Aqui \pm representa a quiralidade dos espinores, sujeitos à condição de Majorana-Weyl.

A ação da da supergravidade $I I$-A é composta por três termos, $S_{N S}, S_{R}$ e $S_{C S}$, representando os dois setores identificados acima e o termo de Chern-Simons, que constitui uma interação entre as formas.

\footnotetext{
${ }^{1} \mathrm{Na}$ seção 6.2 essa construção será utilizada novamente.
} 


$$
\begin{aligned}
S_{N S} & =\frac{1}{2 \kappa^{2}} \int d^{10} x \sqrt{-g} e^{-2 \phi}\left\{R+4 \partial_{\mu} \phi \partial^{\mu} \phi-\frac{1}{2}\left|H_{3}\right|^{2}\right\}, \\
S_{R} & =-\frac{1}{4 \kappa^{2}} \int d^{10} x \sqrt{-g}\left\{\left|F_{2}\right|^{2}+\left|\tilde{F}_{4}\right|^{2}\right\}, \\
S_{C S} & =-\frac{1}{4 \kappa^{2}} \int B_{2} \wedge F_{4} \wedge F_{4} .
\end{aligned}
$$

Aqui,

$$
F_{2}=d A_{1}, \quad F_{4}=d A_{3}, \quad H_{3}=d B_{2}, \quad \tilde{F}_{4}=d A_{3}+A_{1} \wedge H_{3} .
$$

Observa-se em $S_{N S}$ o fator global $e^{-2 \phi}$, que pode ser absorvido por um reescalonamento da métrica para que o termo de Einstein tenha sua forma usual. Tal escolha é denominada referencial de Einstein enquanto, mantido como está, diz-se que a ação está no referencial da corda.

As transformações de supersimetria dos campos presentes em (5.15) não serão aqui analisadas. Uma discussão introdutória pode ser encontrada em [3].

\subsubsection{Supergravidade $I I-\mathrm{B}$}

Ao contrário do tipo $I I$-A, a supergravidade $I I$-B não pode ser obtida por uma redução dimensional da supergravidade $D=11$. Embora o setor NS-NS das duas teorias seja o mesmo, o setor R-R é constituído de formas de grau par: 0-forma, $C_{0} ; 2$-forma, $C_{2}$; e 4-forma, $C_{4}$. É justamente nesse setor que encontra-se a maior dificuldade para a construção de uma ação da supergravidade $I I$-B. Não é possível implementar o vínculo de autodualidade $d C_{4}=F_{5}=\star F_{5}$ de forma trivial, uma vez que o termo cinético seria identicamente nulo.

Para contornar esse inconveniente, será ilustrada a ação que fornece as equações de movimento corretas dos campos desde que o vínculo de autodualidade seja imposto a posteriori ${ }^{2}$.

$$
\begin{aligned}
S_{N S} & =\frac{1}{2 \kappa^{2}} \int d^{10} x \sqrt{-g} e^{-2 \phi}\left\{R+4 \partial_{\mu} \phi \partial^{\mu} \phi-\frac{1}{2}\left|H_{3}\right|^{2}\right\}, \\
S_{R} & =-\frac{1}{4 \kappa^{2}} \int d^{10} x \sqrt{-g}\left\{\left|F_{1}\right|^{2}+\left|\tilde{F}_{3}\right|^{2}+\frac{1}{2}\left|\tilde{F}_{5}\right|^{2}\right\}, \\
S_{C S} & =-\frac{1}{4 \kappa^{2}} \int C_{2} \wedge H_{3} \wedge F_{3} .
\end{aligned}
$$

Aqui,

$$
F_{1}=d C_{0}, \quad F_{3}=d C_{2}, \quad \tilde{F}_{3}=F_{3}-C_{0} \wedge H_{3}, \quad \tilde{F}_{5}=F_{5}-\frac{1}{2} C_{2} \wedge H_{3}+\frac{1}{2} B_{2} \wedge F_{3} .
$$

O vínculo de autodualidade manifesta-se como $\tilde{F}_{5}=\star \tilde{F}_{5}$.

A supergravidade $I I$-B será retomada na seção $6.2 \mathrm{com}$ um estudo contextualizado das equações de movimento e algumas soluções.

\footnotetext{
${ }^{2}$ Como é argumentado em várias referências, esse não é um ponto de vista falho, afinal o interesse aqui não é a teoria quântica mas simplesmente uma ação efetiva, o suficiente para reproduzir a teoria clássica.
} 


\subsection{Comentários}

Notavelmente, o espectro de estados das supercordas não está de acordo com o que é observado na natureza. De fato, mesmo a supersimetria, fundamental na construção de tudo até aqui discutido, não é hoje observada. Desse modo, é necessário apegar-se ao único resultado que aproxima-se minimamente da realidade, que é o espectro não massivo da teoria. Argumentou-se que a corda aberta e a corda fechada poderiam fornecer o fóton e o gráviton em $D=10$ e isso foi aqui levado adiante: foram construídas as ações efetivas da D-branas e da supergravidade. Ações efetivas constituem a abordagem mais amigável para o entendimento da teoria em interação. Principalmente em se tratando de uma teoria complexa como a teoria de supercordas. Entretanto, mesmo esse caminho é trabalhoso e ainda não foi possível extrair qualquer previsão que, hoje, possa testar a teoria.

No final da década de 90 nasceu o que atualmente é a ligação mais promissora entre a teoria de cordas e a natureza. A chamada correspondência gravidade/calibre permitiu vislumbrar pela primeira vez um resultado passível de teste e que, mesmo sem uma prova definitiva, é utilizado para inferir o comportamento de sistemas em regimes até hoje inacessíveias às teorias modernas. Uma introdução básica a esse assunto é realizada no próximo capítulo. 


\section{Capítulo 6}

\section{Aspectos da Correspondência AdS $/$ CFT}

No decorrer do capítulo, são apresentadas algumas propriedades da teoria de $\mathcal{N}=4$ SYM em $D=4$ e da supergravidade $I I$-B, introduzida previamente no capítulo anterior. Com um caráter bastante descritivo, são enfatizadas as simetrias e propriedades comuns às duas teorias, os dois lados da correspondência AdS/CFT, culminando no enunciado da conjecura de Maldacena, em seus três níveis de intensidade.

\section{1 $\mathcal{N}=4$ Super Yang-Mills}

Esta seção introduz alguns dos aspectos mais fundamentais de $\mathcal{N}=4$ super Yang-Mills (SYM), discutindo seu conteúdo de campos, suas simetrias (em particular a simetria conforme), e motivando a expansão $1 / N$.

\subsubsection{O Conteúdo de Campos e a Ação}

Ao construir uma generalização supersimétrica do modelo de Yang-Mills em $D=4$, é interessante analisar o número máximo de supersimetrias que tal modelo comporta.

Seguindo estritamente o raciocínio desenvolvido na subseção C.3.2 do apêndice, a helicidade máxima de uma possível representação para a teoria de super Yang-Mills pura é igual a 1 (é importante lembrar que essa é uma representação não massiva de estados de uma partícula). Dessa forma, a álgebra de criação de helicidade admite no máximo 4 operadores de criação. Isso implica que:

$$
\frac{1}{4} \mathcal{S} \cdot \mathcal{N} \leq 4
$$

em que $\mathcal{S}$ é o tamanho da dimensão da representação espinorial e $\mathcal{N}$ é o número de supersimetrias.

Evidentemente, em $D=10$ há apenas supersimetria $\mathcal{N}=1$, com 16 supercargas associadas a um espinor de Majorana-Weyl. Em $D=4$, a condição de Weyl implica em $\mathcal{S}=4$ e, consequentemente, o número máximo de supersimetrias é $\mathcal{N}=4$ (a condição de Majorana-Weyl não é possivel de ser implementada com esse número de dimensões, portanto esse é verdadeiramente o número máximo de supersimetrias de $\mathcal{N}=4 \mathrm{SYM}$ ). 
Por essa discussão, manifestam-se duas maneiras distintas de se obter o conteúdo de campos do modelo. A primeira é a construção direta da representação pela álgebra de criação dos geradores $Q_{1}^{\mathcal{A}}$ (seguindo a notação da subseção C.3.1), $\operatorname{com} \mathcal{A}=1, \ldots, 4$. Partindo de um vácuo de Clifford de helicidade $-1,|-1\rangle$, obtem-se a representação desejada:

\begin{tabular}{|c|c|c|}
\hline helicidade & representação & \# de estados \\
\hline \hline-1 & $|-1\rangle$ & 1 \\
\hline$-1 / 2$ & $Q_{1}^{\mathcal{A}}|-1\rangle$ & 4 \\
\hline 0 & $Q_{1}^{\mathcal{A}} Q_{1}^{\mathcal{B}}|-1\rangle$ & 6 \\
\hline$+1 / 2$ & $Q_{1}^{\mathcal{A}} Q_{1}^{\mathcal{B}} Q_{1}^{\mathcal{C}}|-1\rangle$ & 4 \\
\hline+1 & $Q_{1}^{4} Q_{1}^{3} Q_{1}^{2} Q_{1}^{1}|-1\rangle$ & 1 \\
\hline
\end{tabular}

Os estados de helicidade \pm 1 estão associados ao campo de calibre $A_{\mu}$; os estados de helicidade $\pm 1 / 2$ estão associados a espinores de Weyl $\lambda^{\mathcal{A}}$; e os escalares são identificados por $X^{i}, \operatorname{com} i=1, \ldots, 6$. Ao todo são

$$
\sum_{n=0}^{4}\left(\begin{array}{l}
4 \\
n
\end{array}\right)=16
$$

graus de liberdade.

Com essa identificação, é necessário entender a simetria $R$ associada aos campos. Naturalmente, o campo de calibre é um singleto de $S U(4)_{R}$. Os espinores $\lambda^{\mathcal{A}}$ constituem vetores na representação fundamental do grupo. Os campos escalares $X^{i}$ correspondem às componentes de um vetor de $S O(6)_{R}$, grupo homomorfo a $S U(4)_{R}$.

A segunda forma de se obter o conteúdo de campos de $\mathcal{N}=4 \mathrm{SYM}$ em $D=4$ é a partir de uma redução dimensional de $\mathcal{N}=1 \mathrm{SYM}$ em $D=10$.

Como foi visto nos capítulos 2, 3 e 4, o espectro não massivo da supercorda aberta na presença de $N$ D-branas dá origem a uma teoria de calibre $U(N)$ supersimétrica. Sua ação efetiva é dada por:

$$
S_{e f}=-\frac{1}{2 g_{Y M}^{2}} \int d^{10} x\left\{\operatorname{tr}\left[F_{\mu \nu} F^{\mu \nu}-2 i \bar{\lambda} \Gamma^{\mu} D_{\mu} \lambda\right]\right\} .
$$

Aqui, $D_{\mu}$ é a derivada covariante, tal que $D_{\mu} \lambda=\partial_{\mu} \lambda+i\left[A_{\mu}, \lambda\right]$ e os índices da represetação adjunta dos campos $A_{\mu}$ e $\lambda$ estão implícitos. As transformações de supersimetria associadas aos campos da ação acima são

$$
\begin{aligned}
\delta A_{\mu} & =-i \bar{\xi} \Gamma_{\mu} \lambda, \\
\delta \lambda & =\frac{1}{2} F_{\mu \nu}, \Gamma^{\mu \nu} \xi,
\end{aligned}
$$

em que $\xi$ é o parâmetro da transformação.

A redução dimensional da ação acima é feita em um torus plano de 6 dimensões (com condições de contorno periódicas em todos os campos). Por conveniência, as dimensões sobre o torus serão indexadas por $i$ enquanto as dimensões restantes serão indexadas por $m$, tal que $i=1, \ldots, 6$ e $m=0, \ldots, 3$. Dessa forma, $A_{\mu}=\left\{A_{m}, X^{i}\right\}, \lambda=\left\{\lambda^{\mathcal{A}}\right\}$ e $\partial_{i}(A, X, \lambda)=0$. A última relação segue da redução dimensional, em que apenas os modos zero na expansão de Fourier dos campos são mantidos. Assim, o campo de calibre em $D=10$ dá orgiem ao campo de calibre em $D=4$ e aos 6 escalares $X^{i}$; enquanto o espinor de Majorana-Weyl em $D=10$ dá origem a 4 espinores de Weyl em $D=4$.

Com o conteúdo de campos descrito, resta introduzir a ação de $\mathcal{N}=4 \mathrm{SYM}$. Analisando a ação efetiva descrita acima e inserindo a decomposição de campos obtida na redução dimensional, verifica-se que o termo $F_{\mu \nu} F^{\mu \nu}$ dá origem a $F_{m n} F^{m n}, D_{m} X^{i} D^{m} X^{i}$ e $\left(X^{i} X^{j}\right)^{2}$, enquanto o bilinear espinorial dá origem 
a termos do tipo $\bar{\lambda}_{\mathcal{A}} \Gamma^{m} D_{m} \lambda^{\mathcal{A}}$ e $\lambda^{\mathcal{A}} \lambda^{\mathcal{B}} X^{i}$. Essa é uma análise qualitativa da decomposição. Quando o procedimento é repetido rigorosamente, obtem-se a ação:

$$
\begin{aligned}
S_{S Y M} & =\int d^{4} x \operatorname{tr}\left\{-\frac{1}{2 g_{Y M}^{2}} F_{m n} F^{m n}+\frac{\theta_{I}}{8 \pi^{2}} F_{m n} \star F^{m n}-i \bar{\lambda}_{\mathcal{A}} \Gamma^{m} D_{m} \lambda^{\mathcal{A}}-D_{m} X^{i} D^{m} X^{i}\right. \\
& \left.+\frac{g_{Y M}^{2}}{2} \sum_{i, j}\left[X^{i}, X^{j}\right]^{2}+g_{Y M}\left(C_{\mathcal{A B}}^{i} \lambda^{\mathcal{A}}\left[\lambda^{\mathcal{B}}, X^{i}\right]+\text { c.c. }\right)\right\} .
\end{aligned}
$$

Aqui, os coeficientes $C_{\mathcal{A B}}^{i}$ estão intimamente relacionados com as matrizes de Dirac nas dimensões reduzidas, mas a sua forma explícita não apresenta relevância alguma no que segue, por isso não será explicitada. O termo $F_{m n} \star F^{m n}$ não é justificado pela redução dimensional descrita. O parâmetro $\theta_{I}$ é denominado ângulo do ínstanton. O ínstanton é um fenômeno não-perturbativo, sendo solução das equações de movimento. O procedimento de dedução dessas equações parte da extremização da ação, mais precisamente, encontrar as equações que determinam os pontos críticos do funcional, sejam mínimos, máximos ou pontos de sela. Ínstantons estão associados a soluções que fornecem uma ação não nula e geralmente aparecem como as primeiras correções da teoria quântica tratadas perturbativamente. Mais detalhes sobre o ínstanton e suas propriedades não serão discutidos aqui.

A seguir são analisadas as simetrias presentes na ação de $\mathcal{N}=4 \mathrm{SYM}$.

\subsubsection{Simetrias}

Uma vez introduzida a ação do modelo é possível analisar as simetrias da teoria.

Por construção, a simetria de calibre é manifesta. Os campos $A_{\mu}, \lambda^{\mathcal{A}}$ e $X^{i}$ transformam-se pela representação adjunta ${ }^{1}$ de $S U(N)$.

Outra simetria presente em $\mathcal{N}=4 \mathrm{SYM}$ é a simetria $\mathrm{R}, S U(4)_{R}$, como foi discutido na seção anterior. Tal simetria decorre diretamente da existência de supersimetria $\mathcal{N}=4$.

A simetria de super Poincaré, isto é, a simetria de Poincaré mais a supersimetria, aparentemente esgota as simetrias externas da ação (6.1).

Evocando a generalização supersimétrica do teorema de Coleman-Mandula [20, 21], fica evidente uma brecha em uma de suas condições. As simetrias do espaço-tempo podem incluir algo mais em relação ao grupo de super Poincaré e a ausência de estados massivos sugere uma invariância de escala.

Examinando a ação (6.1) cuidadosamente, isso é verificado imediatamente. $\theta_{I}$ e $g_{Y M}$ possuem dimensão nula, confirmando a simetria de escala no nível clássico. Aprofundando a análise, verifica-se que a invariância de escala é também uma simetria no nível quântico. Tal conclusão decorre do anulamento da função beta, que garante a independência da constante acoplamento em relação à escala de renormalização.

O grupo que inclui o grupo de Poincaré e a invariância de escala é o grupo conforme. Algumas de suas propriedades são rapidamente introduzidas no apêndice E, incluindo a definição dos geradores.

Ao estender a álgebra com a inclusão da supersimetria é obtido o grupo superconforme. Em particular, o grupo de simetria resultante de $\mathcal{N}=4 \mathrm{SYM}$ é o supergrupo $S U(2,2 \mid 4)$, uma vez que $S O(2,4)$ é homomorfo a $S U(2,2)$. Como já foi discutido, o supergrupo $S U(2,2 \mid 4)$ permanece como simetria da teoria quântica. O gerador de simetria superconforme é representado pelo espinor $S^{\mathcal{A}}$ e pertence à mesma representação espinorial dos geradores de supersimetria. Sua existência deve-se ao fato de que a simetria conforme especial e a translação não comutam. Grosseiramente, os geradores superconformes

\footnotetext{
${ }^{1}$ No modelo padrão, por exemplo, os quarks possuem carga de cor, pertencendo à representação fundamental de $S U$ (3), enquanto aqui os férmions são os parceiros supersimétricos do campo de calibre e, naturalmente, pertencem à representação adjunta.
} 
estão para os geradores de simetria conforme especial assim como os geradores de supersimetria estão para os geradores de translação.

As dimensões dos supergeradores são, em unidades de massa ${ }^{2}$,

$$
\left[Q^{\mathcal{A}}\right]=\frac{1}{2}, \quad\left[S^{\mathcal{A}}\right]=-\frac{1}{2}
$$

e junto a (E.4) constituem uma gradação natural da álgebra superconforme.

Além da simetria superconforme há ainda uma simetria discreta na teoria quântica, constituída por uma combinação do acoplamento $g_{Y M}$ e do ângulo do ínstanton $\theta_{I}$, dando origem a um parâmetro complexo de acoplamento

$$
\varsigma=\frac{\theta_{I}}{2 \pi}+i \frac{4 \pi}{g_{Y M}^{2}} .
$$

Pelo modo como o ângulo do ínstanton aparece na teoria quântica $\left(e^{i n \theta_{I}}, n \in \mathbb{Z}\right)$, a teoria é invariante por $\theta_{I} \rightarrow \theta_{I}+2 \pi$ ou, consequentemente, $\varsigma \rightarrow \varsigma+1$. Junto a ela, existe a simetria $\varsigma \rightarrow-1 / \varsigma$, conjecturada por C. Montonen e D. Olive. Essas duas simetrias constituem o grupo de dualidade S, $S L(2, \mathbb{Z})$, gerado pelas transformações ${ }^{3}$

$$
\varsigma \rightarrow \frac{a \varsigma+b}{c \varsigma+d}
$$

De forma sucinta, aqui foram descritas as simetrias clássicas e quânticas de $\mathcal{N}=4 \mathrm{SYM}$. A simetria superconforme é utilizada no que segue para classificar alguns dos operadores de interesse na teoria.

\subsubsection{Multipletos Superconformes}

Uma vez que a gradação da álgebra conforme é bastante objetiva, ela pode ser utilizada para classificar multipletos superconformes de operadores. Isso será feito aqui de acordo com [26].

Para isso, é interessante que os operadores sejam polinomiais nos campos fundamentais, possuindo, portanto, dimensão definida. Além disso, é natural considerar operadores invariantes de calibre. Examinando as possibilidades, os operadores em questão devem constituir-se de um produto de potências inteiras positivas de $X^{i}, \lambda^{\mathcal{A}}, F_{\mu \nu}$ e possivelmente derivadas covariantes. Como esses campos possuem uma dimensão não renormalizada bem definida e positiva, qualquer operador com os requisitos acima também possuirá essas características, além da identidade $\mathbb{I}$, evidentemente, que possui dimensão nula.

Um multipleto supersimétrico é um conjunto de operadores que são mapeados um no outro por transformações de supersimetria. Assim, na classificação superconforme, há apenas um operador $\mathcal{O}_{(1)}$ que possui a menor dimensão do multipleto. Os outros operadores $\mathcal{O}_{(k)}$ do multipleto são chamados de descendentes de $\mathcal{O}_{(1)}$.

Como os geradores superconformes $S$ possuem dimensão $-1 / 2$, sua sucessiva aplicação em um dado operador leva, em algum momento, a um operador de dimensão negativa, o que é impossível em uma teoria unitária (isso pode ser visto através de uma possível não-renormalizabilidade por contagem de potências). Ou seja, o operador de menor dimensão do multipleto deve ser aniquilado por $S$, o que, em linguagem operatorial, é traduzido por

$$
\left[S, \mathcal{O}_{(1)}\right]_{ \pm}=0
$$

Aqui, [, $]_{ \pm}$é uma comutação (anticomutação), se $\mathcal{O}_{(1)}$ é bosônico (fermiônico). Se um dado operador satisfaz à equação acima, então é denominado operador superconforme primário. Além disso, se

$$
\mathcal{O}_{(i)}=\left[Q, \mathcal{O}_{(j)}\right]
$$

\footnotetext{
${ }^{2}$ Aqui é utilizado o sistema natural de unidades, em que $c=\hbar=1$.

${ }^{3} \mathrm{O}$ nome dualidade $\mathrm{S}$ vem de uma das dualidades da teoria de cordas, citada no início da seção 5.3 , que troca os regimes de acoplamento (forte e fraco) entre duas teorias ou na mesma teoria.
} 
então $\mathcal{O}_{(i)}$ é chamado de descendente superconforme de $\mathcal{O}_{(j)}$.

Com essa classificação definida, é fácil determinar quais as possibilidades de operadores primários da teoria: um operador superconforme primário não pode ser descendente de qualquer outro, de modo que, ao analisar as transformações de supersimetria dos campos,

$$
\begin{aligned}
\{Q, \lambda\} \sim F+[X, X], & {[Q, X] \sim \lambda, } \\
\{Q, \tilde{\lambda}\} \sim D X, & {[Q, F] \sim D \lambda, }
\end{aligned}
$$

a única possibilidade de operadores conformes primários é

$$
\operatorname{tr}\left\{X^{i} X^{j} \ldots X^{k}+(\text { simetrização })\right\}
$$

e seus produtos. O traço garante a invariância de calibre, a simetrização garante que não há operações de comutação e, evidentemente, as derivadas covariantes $D X$ não são permitidas.

Como o grupo de calibre é $S U(N)$, apenas o produto de dois ou mais escalares é possível, sendo os mais simples

$$
\begin{aligned}
\sum_{i} \operatorname{tr}\left\{X^{i} X^{i}\right\} & \rightarrow \text { multipleto de Konishi, } \\
\operatorname{tr}\left\{X^{\{i} X^{j\}}\right\} & \rightarrow \text { multipleto de supergravidade. }
\end{aligned}
$$

Aqui, $\{i j\}$ corresponde à parte simétrica de $S O(6)$ de traço nulo. Esses operadores serão mencionados novamente na seção 6.3, ao introduzir a correspondência AdS/CFT.

Representações antissimétricas de $S O(6)$ também são possíveis, mas a construção é através de produtos dos operadores (6.4), chamados operadores de traço múltiplo.

\subsubsection{Expansão $\frac{1}{N}$}

Seguindo o desenvolvimento proposto em [22], um dos aspectos mais evidentes da dualidade calibre/gravidade é a chamada expansão $1 / N$, introduzida em [23].

A densidade lagrangeana de uma teoria de calibre com campos que transformam-se sob a representação adjunta possui, em geral, a seguinte constituição:

$$
\mathcal{L} \sim \frac{1}{g^{2}} \operatorname{tr}\left\{d \Phi_{A} d \Phi_{A}+c^{A B C} \Phi_{A} \Phi_{B} \Phi_{C}+d^{A B C D} \Phi_{A} \Phi_{B} \Phi_{C} \Phi_{D}\right\} .
$$

Aqui, os índices identificam os campos da teoria, designando espinores, campos vetoriais, escalares, etc. A constante de acoplamento como um fator global decorre de um simples reescalonamento dos campos. Define-se aqui $\lambda \equiv g^{2} N$, em que $N$ é o grau do grupo de calibre ( $\left.S U(N), U(N), S O(N), O(N)\right)$. Desse ponto em diante serão considerados apenas os grupos unitários.

Nessas teorias, a expansão $1 / N$ constitui uma alternativa à teoria de perturbação usual, em termos da constante de acoplamento. Entretanto, o fato que chama mais a atenção em tal abordagem é a semelhança com a teoria de cordas em interação.

Usualmente, os campos da representação adjunta carregam o índice de identificação dos geradores do grupo de calibre, $T^{a}$. Na proposta de t'Hooft, os campos adjuntos passam a ser representados como um produto direto de uma representação fundamental e uma antifundamental, ou seja,

$$
\Phi^{a} \rightarrow \sum_{a} \Phi^{a} T^{a} \equiv \Phi^{i}{ }_{j}
$$


Dessa forma, é introduzida uma alternativa à diagramação de Feynman. Agora, os propagadores são representados por uma linha dupla com orientações opostas, associadas às representações fundamental e antifundamental (no caso dos grupos ortogonais as linhas não são orientadas, uma vez que não é possível distingui-las).

Ao analisar, então, o propagador dos campos, verifica-se que

$$
\left\langle\Phi_{j}^{i} \Phi_{l}^{k}\right\rangle \propto\left(\delta_{l}^{i} \delta_{j}^{k}-\frac{1}{N} \delta_{j}^{i} \delta_{l}^{k}\right)
$$

para $S U(N)$. Tal constatação é derivada das propriedades do grupo ao realizar a decomposição matricial dos geradores. O termo com $1 / N$ na equação acima está associado à nulidade do traço dos geradores do grupo e não existe para o grupo $U(N)$.

No limite $N \rightarrow \infty, U(N) \sim S U(N)$, de modo que, pela estrutura de índices, justifica-se a notação de linha dupla. Como $U(N)=U(1) \otimes S U(N)$, esse limite corresponde ao acoplamento de um campo de calibre $U(1)$ a $S U(N)$.

É interessante analisar a estrutura de diagramas dessa abordagem. Na notação de linha dupla, os vértices possuem a seguinte representação:
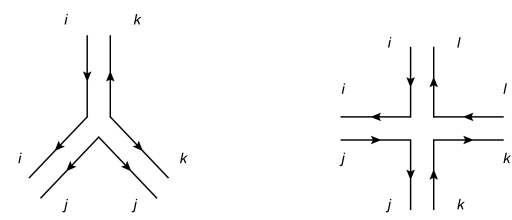

Figura 6.1: Representação dos vértices triplo e quádruplo.

Os diagramas da teoria são uma composição dos vértices acima e sua contribuição perturbativa pode ser compreendida em termos de $N$ e $\lambda$ : cada vértice $(\mathrm{V})$ contribui com um fator $g^{2}=\lambda / N$; cada propagador $(\mathrm{P})$ contribui com $N / \lambda$; e a cada loop $(\mathrm{L})$ há uma soma sobre os índices da representação, de modo que sua contribuição é $N$. Desse modo, um dado diagrama (V,P,L) apresenta potências em $N$ e $\lambda$ muito bem definidas. De um ponto de vista geométrico, ao identificar as linhas de propragadores com arestas (A) e os loops com faces (F) de um polígono, a contribuição de cada diagrama pode ser expressa por

$$
(V, A \equiv P, F \equiv L) \rightarrow N^{\chi} \lambda^{A-V},
$$

em que $\chi=V+F-A$ é a característica de Euler da superfície ${ }^{4}$. Assim, uma dada função de Green $G$ na teoria apresenta a seguinte estrutura perturbativa:

$$
G=\sum_{\chi} N^{\chi} f_{\chi}(\lambda)
$$

Para superfícies fechadas orientadas ${ }^{5}, \chi=2-2 n_{h}$, sendo $n_{h}$ o genus da superfície. Portanto:

$$
G=\sum_{n_{h}=0}^{\infty} N^{2-2 n_{h}} f_{n_{h}}(\lambda) .
$$

Por essa análise, os diagramas que contribuem para a função de Green no limite $N \rightarrow \infty$ (conhecido como limite de t'Hooft), com $\lambda$ fixo, são aqueles em que $\chi=2$, ou seja, apenas os diagramas ditos planares.

\footnotetext{
${ }^{4}$ Uma discussão bastante simples e intuitiva sobre essa construção e a característica de Euler de superfícies poligonais pode ser encontrada em um dos apêndices de [24].

${ }^{5}$ A construção de tais superfícies é possível devido à orientação da linha dupla. Por essa razão, os grupos ortogonais não podem ser associados, em geral, a superfícies orientáveis na expansão diagramática.
} 
Essa análise é estendida diretamente para $\mathcal{N}=4 \mathrm{SYM}$. E esse é, afinal, um dos pontos a serem discutidos na seção 6.3 em direção à correspondência.

A seguir, discute-se a supergravidade $I I$-B.

\subsection{Supergravidade $I I$-B}

Nesta seção são estudadas algumas propriedades da supergravidade $I I$-B. As equações de movimento na presença de branas são deduzidas e um conjunto específico de soluções é indicado. Especial atenção é dada ao caso da D3-brana e a geometria $A d S_{5} \times S^{5}$. As referências base do texto são [22, 26].

\subsubsection{Simetrias}

O conteúdo de campos da supergravidade $I I$-B e sua ação já foram introduzidos na seção 5.3. Agora, suas simetrias devem ser analisadas.

A principal simetria da supergravidade é comum, evidentemente, à teoria da gravidade ordinária, descrita pela ação de Einstein-Hilbert, ou seja, é a transformação geral de coordenadas.

Sem dúvida alguma, a supersimetria está presente. Além disso, a teoria é quiral (suas duas supersimetrias possuírem a mesma quiralidade). Como foi apontado na seção 5.3, o interesse na supergravidade II-B concentra-se principalmente no regime clássico, de modo que os campos fermiônicos são nulos. As transformações de supersimetria do dilatino $\Phi_{ \pm}$e do gravitino $\Psi_{\mu \pm}$ são, em primeira aproximação,

$$
\begin{aligned}
\delta \Phi & =\frac{1}{2}\left(\partial_{\mu} \phi-i e^{-\phi} \partial_{\mu} C_{0}\right) \Gamma^{\mu} \xi+\frac{1}{4}\left(i e^{\phi} \tilde{F}^{(3)}-H^{(3)}\right) \xi^{*} \\
\delta \Psi_{\mu} & =\left(D_{\mu}+\frac{i}{8} e^{\phi} F^{(1)} \Gamma_{\mu}+\frac{i}{16} e^{\phi} \tilde{F}^{(5)} \Gamma_{\mu}\right) \xi-\frac{1}{8}\left(2 H_{\mu}^{(3)}+i e^{\phi} \tilde{F}^{(3)} \Gamma_{\mu}\right) \xi^{*} .
\end{aligned}
$$

Aqui, os dois dilatinos e os dois gravitinos foram organizados em espinores de Weyl, por isso a notação complexa. $F^{(n)}$ e $F_{\mu}^{(n)}$ são definidos de acordo com (5.13). Para $\delta \Phi=\delta \Psi_{\mu}=0$, são obtidos os espinores de Killing, responsáveis por manter a solução clássica.

Com a supersimetria $\mathcal{N}=2$ vem a simetria $\mathrm{R}$. Como em $D=10$ as cargas de supersimetria são espinores de Majorana-Weyl (ou seja, uma representação quiral real) o grupo de simetria R é $S O(2) \sim$ $U(1)$.

No capítulo 4 foi levantada a possibilidade de uma simetria associando os campos dos setores NS-NS e R-R. Isso é realmente observado, ao menos no nível não massivo. Essa simetria foi identificada pela primeira vez em [25], sendo associada ao grupo $S U(1,1)$.

Ao analisar o conteúdo de campos da supergravidade $I I$-B, são observadas as seguintes formas:

\begin{tabular}{|c|c|c|c|}
\hline forma & setor & acoplamento elétrico & acoplamento magnético \\
\hline \hline$B_{2}$ & NS-NS & 1-brana (F-corda) & 5-brana (NS5-brana) \\
\hline$C_{0}$ & R-R & (-1)-brana (D-ínstanton) & 7-brana \\
\hline$C_{2}$ & R-R & 1-brana (D-corda) & 5-brana \\
\hline$C_{4}$ & R-R & 3-brana & 3-brana \\
\hline
\end{tabular}

Tal simetria está longe de ser evidente, sendo estabelecida do como segue. As 2-formas $B_{2}$ e $C_{2}$ são organizadas em um dubleto de $S L(2, \mathbb{R})$, homomorfo à $S U(1,1), \operatorname{com} B_{2}^{(1)} \equiv B_{2}$ e $B_{2}^{(2)} \equiv C_{2}$. A 
transformação é dada por

$$
B \rightarrow B^{\prime}=\Lambda B, \quad \Lambda=\left(\begin{array}{cc}
a & b \\
c & d
\end{array}\right) .
$$

Por essa transformação, a $C_{0}$ e $\phi$, reorganizados em um campo complexo $\varsigma$,

$$
\varsigma=C_{0}+i e^{-\phi}
$$

também são modificados, tal que

$$
\varsigma \rightarrow \frac{a \varsigma+b}{c \varsigma+d}
$$

$\varsigma$ é conhecido como campo do axion-dílaton. A ação (5.16) pode ser reescrita para tornar essa simetria manifesta e uma forma de se fazer isso encontra-se em [3].

Apesar de presente na teoria clássica, nota-se claramente que a teoria quântica não mantém tal simetria. A razão disso é fundamentada no que foi discutido no capítulo 5, a condição de quantização DNT, (5.4), que implica que as cargas elétricas e magnéticas das branas são quantizadas. Evidentemente, a simetria $S L(2, \mathbb{R})$ viola tal condição. A menos que o grupo seja, de alguma forma, restrito, a simetria é completamente perdida no nível quântico. Em outras palavras, os parâmetros da transformação, $a, b$, $c$ e $d$ devem ser inteiros e, de fato, o grupo de simetria da teoria quântica é $S L(2, \mathbb{Z})^{6}$.

Naturalmente, a transformação do campo $\varsigma$ constitui uma dualidade S, uma vez que o campo do dílaton está associado à constante de acoplamento das cordas. A semelhança entre (6.3) e (6.9) é clara, sendo este um dos pontos a serem discutidos na seção 6.3 sobre a correspondência.

As simetrias acima descritas constituem todas as simetrias da ação (5.16). É sabido, entretanto, que as equações de movimento podem não apresentar as simetrias de sua ação. O grupo de difeomorfismos, por exemplo, sofre severas restrições e, em geral, fica reduzido às isometrias de uma dada métrica. Isso é verificado a seguir, com as soluções da supergravidade na presença de branas. Na próxima subseção são obtidas as equações de movimento associadas a ação (5.16) e introduzidas as soluções de branas extremas.

\subsubsection{Equações de Movimento e Soluções Extremas}

A ação (5.16) está escrita no referencial da corda e é conveniente trazê-la para o referencial de Einstein. Para isso é realizada uma transformação conforme $g_{\mu \nu} \rightarrow e^{a \phi} g_{\mu \nu}$, sendo a constante $a$ escolhida convenientemente. Desse modo, após uma série de manipulações, verifica-se que

$$
\sqrt{-g} e^{-2 \phi} R \rightarrow \sqrt{-g}\left[R-\frac{(D-1)(D-2)}{4} a^{2}\left(\partial^{\mu} \phi \partial_{\mu} \phi\right)-a(D-1) \square \phi\right] \times \exp \left\{\left(\frac{a D}{2}-a-2\right) \phi\right\} .
$$

Em $D=10$, recupera-se o termo de Einstein para $a=1 / 2$ e o último termo entre colchetes na expressão acima corresponde a uma derivada total, sendo, portanto, desprezado.

Além disso, é necessário analisar como a transformação conforme afeta, por exemplo, o termo cinético das $(p+1)$-formas:

$$
\left|F_{p+1}\right|^{2}=g^{\mu_{1} \nu_{1}} \ldots g^{\mu_{n} \nu_{n}} F_{\mu_{1} \ldots \mu_{p+21}} F_{\nu_{1} \ldots \nu_{p+2}} \rightarrow e^{-a(p+2) \phi}\left|F_{p+2}\right|^{2} .
$$

Assim, a parte bosônica da ação da supergravidade $I I$-B no referencial de Einstein, a menos do termo de Chern-Simons, é

$$
S_{I I-B}=\frac{1}{2 \kappa^{2}} \int d^{10} x \sqrt{-g}\left\{R-\frac{1}{2} \partial_{\mu} \phi \partial^{\mu} \phi-\frac{1}{2} e^{-\phi}\left|H_{3}\right|^{2}-\frac{1}{2} e^{-2 \phi}\left|F_{1}\right|^{2}-\frac{1}{2} e^{-\phi}\left|F_{3}\right|^{2}-\frac{1}{4}\left|F_{5}\right|^{2}\right\} .
$$

\footnotetext{
${ }^{6}$ Existe todo um desenvolvimento da teoria nessa direção, nas chamadas cordas $(p, q)$. Nessa caso, $p$ é a carga da F-corda e $q$ é a carga da D-corda, introduzidas previamente na tabela de formas e seus acoplamentos.
} 
Para simplificar a obtenção das equações de movimento sem, entretanto, prejudicar sua generalidade, será considerada a presença de apenas uma $(p+1)$-forma, ou seja ${ }^{7}$,

$$
S=\frac{1}{2 \kappa^{2}} \int d^{10} x \sqrt{-g}\left\{R-\frac{1}{2} \partial_{\mu} \phi \partial^{\mu} \phi-\frac{1}{2} e^{\frac{3-p}{2} \phi}\left|F_{p+2}\right|^{2}\right\} .
$$

O fator de normalização do termo cinético da $(p+1)$-forma é apenas uma questão de escolha e pode ser absorvido por um reescalonamento do campo.

As equações de movimento são obtidas sem maiores dificuldades.

- $g_{\mu \nu}$ :

$$
R_{\mu \nu}-\frac{1}{2} g_{\mu \nu}\left[R-\frac{1}{2} \partial_{\rho} \phi \partial^{\rho} \phi-\frac{1}{2} e^{\frac{5-p}{2} \phi}\left|F_{p}\right|^{2}\right]=\frac{1}{2} \partial_{\mu} \phi \partial_{\nu} \phi+\frac{p}{2} e^{\frac{3-p}{2} \phi} F_{\mu \mu_{2} \ldots \mu_{p+2}} F_{\nu}{ }^{\mu_{2} \ldots \mu_{p+2}}
$$

- $\phi$ :

$$
\partial_{\mu}\left(\sqrt{-g} g^{\mu \nu} \partial_{\nu} \phi\right)=\sqrt{-g}\left(\frac{3-p}{2}\right) e^{\frac{3-p}{2} \phi}\left|F_{p+2}\right|^{2}
$$

- $C_{p-1}$ :

$$
\partial_{\mu}\left(\sqrt{-g} e^{\frac{5-p}{2}} \phi F^{\mu \mu_{2} \ldots \mu_{p+2}}\right)=0 .
$$

Para a $(p+1)$-forma existe ainda a identidade de Bianchi:

$$
\partial_{\left[\mu_{1}\right.} F_{\left.\mu_{2} \ldots \mu_{p+3}\right]}=0
$$

O processo de dedução aqui mostrado não é válido para $C_{4}$, uma vez que a condição de autodualidade não foi imposta. As equações de movimento são ligeiramente alteradas, com $F_{5}$ sendo substituído por $F_{5}+\star F_{5}$.

Como acontece, em geral, nas teorias de gravitação, as equações de movimento são altamente não lineares, permitindo a construção de soluções exatas apenas em alguns casos que envolvem simetrias chave. O interesse no texto repousa sobre as soluções de branas, também conhecidas como soluções extensas. São sólitons da teoria clássica e sua dinâmica não é levada em conta, apenas seu impacto na geometria do espaço-tempo. Ou seja, são soluções estáticas. Em $D=10$, a presença de uma $p$-brana sugere que essa geometria deve possuir o seguinte o grupo de simetria [26]

$$
\mathbb{R}^{p+1} \times S O(p) \times S O(9-p) .
$$

O primeiro grupo corresponde à simetrias de translação no tempo e nas direções da brana (considerada uniforme). O segundo responde pelas simetria de rotação sobre a brana, enquanto o último grupo está associado à simetria de rotação nas direções transversais.

Antes de prosseguir, é conveniente fazer uma decomposição dos índices do espaço-tempo:

$$
\mu=0, \ldots, 9 \rightarrow\left\{\begin{array}{l}
m=0, \ldots, p \\
i=p+1, \ldots, 9
\end{array}\right.
$$

Uma dedução bastante completa de soluções extensas em supergravidade pode ser encontrada em [27]. A base da dedução ali contida são os espinores de Killing, associados precisamente às simetrias acima descritas.

\footnotetext{
${ }^{7}$ Nesse caso, as ações simplificadas das teorias $I I$-A e $I I$-B são muito parecidas, embora uma contenha formas de grau par e outra formas de grau ímpar.
} 
Admitindo que a $p$-brana carregue uma carga de Ramond-Ramond (R-R) $Q_{p}$, são obtidas as chamadas soluções negras (descritas brevemente em [22] e nas referências ali contidas). Seu nome é justificado pela semelhança com o buraco negro de Reissner-Nordstrom, também descrito por dois parâmetros, a saber, sua massa e sua carga.

As soluções extremas constituem um caso particular das soluções negras e são descritas por apenas um parâmetro, igualando, nesse caso, massa e carga. Ao fazer isso, obtem-se automaticamente a simetria de Lorentz $S O(1, p)$.

Os Ansätze para obter tal solução são os seguintes:

- Métrica:

$$
d s^{2}=B^{2}(r)\left(d x^{m} d x^{n} \eta_{m n}\right)+F^{2}(r)\left(d r^{2}+r^{2} d \Omega_{7-p}^{2}\right) .
$$

Para ser mais preciso, o Ansatz inicial é mais geral e, após algumas etapas do cálculo, o resultado acima é atingido.

- Tensor do Campo:

$$
F_{0 \mu_{1} \ldots \mu_{p} r}=\epsilon_{\mu_{1} \ldots \mu_{p}} \partial_{r} E(r) .
$$

Esse é o chamado Ansatz elétrico.

- Dílaton: $\phi=\phi(r)$.

Nota-se aqui que as coordenadas $x^{i}$ foram reescritas em coordenadas esféricas, tal que $r^{2}=x^{i} x^{i}$, deixando evidente tal simetria nas direções transversais à $p$-brana.

Substituindo as hipóteses acima nas equações de movimento e impondo a condição de extremismo, são obtidas as soluções:

$$
\begin{aligned}
d s^{2} & =H^{\frac{p-7}{8}}\left(d x^{m} d x^{n} \eta_{m n}\right)+H^{\frac{p+1}{8}}\left(d r^{2}+r^{2} d \Omega_{8-p}^{2}\right), \\
e^{\phi} & =H^{\frac{3-p}{4}} \\
F_{0 \mu_{1} \ldots \mu_{p} r} & =\epsilon_{\mu_{1} \ldots \mu_{p}} \partial_{r}\left(\frac{1}{H}\right),
\end{aligned}
$$

em que

$$
H(r)=1-\frac{L^{7-p}}{r^{7-p}} .
$$

$L$ é a única constante dimensional das soluções e deve, portanto, estar associada ao comprimento $l_{s}$ da corda e, possivelmente, à constante de acoplamento $g_{s}$ e à carga $Q_{p}[26]$.

Para entender a importância das soluções extremas, é necessário discorrer sobre o efeito da quebra de invariância translacional na conservação das supercargas, os geradores de supersimetria. Intuitivamente, se houver supercargas conservadas, estas devem satisfazer à

$$
\left\{Q_{A}, Q_{B}^{\dagger}\right\}=P_{m}\left(\Gamma^{m} \Gamma^{0}\right)_{A B},
$$

indicando que apenas parte dos geradores de translação são geradores de simetria. Aqui, presume-se a simetria de Lorentz $S O(1, p)$.

A pergunta natural nesse ponto é: a partir da álgebra de supersimetria, é possível identificar qual é a combinação de supercargas que sobrevive à quebra da invariância translacional? Para ilustrar esse ponto, será considerada uma álgebra de supersimetria $\mathcal{N}=2$ qualquer sem carga central ${ }^{8}$. De forma geral,

$$
\left\{Q_{A}^{\mathcal{A}}, \bar{Q}_{B \mathcal{B}}\right\}=\delta_{\mathcal{B}}^{\mathcal{A}}\left(P_{m} \Gamma_{A B}^{m}+P_{i} \Gamma_{A B}^{i}\right) .
$$

\footnotetext{
${ }^{8}$ A ausência da carga central não é uma condição, sendo usada apenas para manter o cálculo mais enxuto.
} 
Supondo que as supercargas conservadas sejam uma combinaçao linear de $Q^{1}$ e $Q^{2}$, é sempre possível, por transformações de similaridade, reescrevê-las como $Q=Q^{1}+M Q^{2}$, sendo $M$ uma matriz a ser determinada. Tal combinação implica em

$$
\left\{Q_{A}, Q_{B}^{\dagger}\right\}=P_{m}\left(\Gamma^{m} \Gamma^{0}+M \Gamma^{m} \Gamma^{0} M^{\dagger}\right)_{A B}+P_{i}\left(\Gamma^{i} \Gamma^{0}+M \Gamma^{i} \Gamma^{0} M^{\dagger}\right)_{A B} .
$$

Portanto, para reproduzir (6.15) (a menos de um fator de normalização), $M$ deve satisfazer a

$$
\begin{aligned}
M \Gamma^{m} \Gamma^{0} M^{\dagger} & =\Gamma^{m} \Gamma^{0}, \\
M \Gamma^{i} \Gamma^{0} M^{\dagger} & =-\Gamma^{i} \Gamma^{0} .
\end{aligned}
$$

Analisando as possibilidades de construção de $M$ por produtos de matrizes de Dirac, verifica-se, por consistência, que envolve o produto de apenas matrizes $\Gamma^{m}$ ou apenas matrizes $\Gamma^{i}$. Como espera-se que a simetria de Lorentz sobre a $p$-brana seja mantida, qualquer combinação linear que envolva o produto de $d<p+1$ matrizes $\Gamma^{m}$ ou $d<D-p-1$ matrizes $\Gamma^{i}$ quebra tal invariância. Portanto,

$$
M(p)=\prod_{m=0}^{p} \Gamma^{m} .
$$

É fácil demonstrar que $M^{\dagger} M=\mathbb{I}$. Além disso,

$$
\left\{M(p), \Gamma^{i} \Gamma^{0}\right\}=\left[M(p), \Gamma^{m} \Gamma^{0}\right]=0,
$$

precisamente as relações desejadas. Assim, a combinação procurada é

$$
Q=Q^{1}+\Gamma^{0} \Gamma^{1} \ldots \Gamma^{p} Q^{2} .
$$

A solução extrema satisfaz os requisitos do cálculo acima. Mantém a simetria de Lorentz sobre a brana e quebra a invariância translacional nas direções transversais. Notavelmente, apenas metade das supercargas são conservadas e a solução extrema é chamada $\frac{1}{2}$ BPS. Como é apresentado no apêndice C, a saturação do vínculo BPS acontece quando a carga central da álgebra (nesse caso identificada com a carga R-R) é igual à massa da $p$-brana (sua tensão ou densidade de energia). Em outras palavras, a condição de extremismo corresponde à saturação do vínculo BPS.

Aqui fica evidente a semelhança com um outro objeto extenso, associado às supercordas abertas, a $\mathrm{D} p$-brana, também um estado $\frac{1}{2} \mathrm{BPS}$.

\subsubsection{A 3-brana e a Geometria $A d S_{5} \times S^{5}$}

Examinando (6.14) observa-se uma solução que é, no mínimo, intrigante: a 3-brana. Nota-se que $p=3$ é a condição de desacoplamento do campo do dílaton e da forma $C_{4}$, como pode ser visto em (6.11). Mais relevante ainda, é o fato de que $\phi$ é constante. Consequentemente, lembrando das simetrias descritas na subseção 6.2.1, o campo do axion também é constante. Além disso, a 3-brana extrema possui a simetria de Poincaré em quatro dimensões, sendo que, em $r=0$, a métrica sobre a brana corresponde à métrica plana.

Antes de prosseguir a análise, é bastante útil discutir a validade da aproximação de baixas energias, especialmente o caso da 3-brana. Desse modo, as conclusões serão mais sólidas e transparentes.

O único parâmetro livre da teoria de cordas é $l_{s}$. O acoplamento $g_{s}$ está associado ao valor esperado do campo do dílaton através de $g_{s}=e^{\langle\phi\rangle}$. Estes são os dois possíveis parâmetros de uma expansão perturbativa. 
Há ainda uma escala de comprimento fundamental, o comprimento de Planck, $l_{P}$. Em unidades naturais, é definido em função da constante de acoplamento gravitacional $\kappa_{D}$ em $D$ dimensões:

$$
2 \kappa_{D}^{2}=\frac{1}{2 \pi}\left(2 \pi l_{P}\right)^{D-2} .
$$

No contexto da supergravidade em $D=10, l_{s}$ é a escala de comprimento no referencial da corda, enquanto $l_{P}$ é a escala no referencial de Einstein. Devido ao escalonamento da métrica $g_{\mu \nu} \rightarrow e^{a \phi} g_{\mu \nu}$, as duas escalas estão associadas por

$$
l_{P}^{2}=\sqrt{g_{s}} l_{s}^{2} \Rightarrow l_{p}=g_{s}^{\frac{1}{4}} l_{s}
$$

Além disso, ao relembrar as equações (5.3), verifica-se imediatamente que

$$
L^{7-p}=g_{s} l_{s}^{7-p} 2^{5-p} \pi^{\frac{5-p}{2}} \Gamma\left(\frac{7-p}{2}\right) Q_{p} .
$$

Essa relação é facilmente obtida ao considerar volumes esféricos em dimensões arbitrárias (no caso, em $(8-p)$ dimensões $)^{9}$.

O parâmetro $L$ pode ser associado diretamente à curvatura $R$ das soluções dadas em (6.14). Particularizando para o caso da 3-brana é possível verificar, grosseiramente, que $R \sim L^{-2}$. De acordo com o espectro das supercordas, $l_{s} \rightarrow 0$ torna os estados massivos inacessíveis a baixas energias. Assim, correções da supergravidade ocorrem, em uma expansão perturbativa, acompanhadas de potências positivas de $l_{s}^{2}$. Portanto, a condição

$$
R l_{s}^{2} \ll 1 \Rightarrow L \gg l_{s}
$$

é necessária para que as soluções descrevam o regime de baixas energias.

Seguindo [22], a constante de acoplamento $g_{s}$ deve ser pequena, de tal modo que não há correções da teoria em interação e apenas os diagramas planares contribuem (diagramas de nível de árvore na expansão perturbativa). Se $g_{s}>1$, basta fazer uma dualidade S. Com as condições descritas, $g_{s}<1 \mathrm{e}$ $L \gg l_{s}$, e recordando a relação $(6.17)$, obtem-se:

$$
l_{P}<l_{s} \ll L \Rightarrow 1 \ll g_{s} Q_{3}<Q_{3} .
$$

Esse é o regime de validade clássico da solução extrema da 3-brana, sendo mapeado nas soluções clássicas por $r \rightarrow 0$, ou seja $L / r \gg 1$. Ao tomar esse limite nas soluções (6.14),

$$
d s^{2}=\frac{r^{2}}{L^{2}}\left(d x^{m} d x^{n} \eta_{m n}\right)+\frac{L^{2}}{r^{2}}\left(d r^{2}+r^{2} d \Omega_{5}^{2}\right) .
$$

Com uma redefinição, $u \equiv L^{2} / r$, obtem-se

$$
d s^{2}=L^{2}\left[\frac{1}{u^{2}} d x^{m} d x^{n} \eta_{m n}+\frac{d u^{2}}{u^{2}}+d \Omega_{5}^{2}\right]
$$

A métrica acima é bastante conhecida e corresponde ao espaço $A d S_{5} \times S^{5}$. O subespaço $S^{5}$ não apresenta nenhuma sutileza. Seu raio, $L$, é facilmente identificado na métrica e seu grupo de isometria é $S O(6)$. O subespaço $A d S_{5}$, por outro lado, é mais interessante. Dentre as várias propriedades deste espaço $^{10}$, duas se destacam, ao menos no nível de discussão aqui proposto: seu grupo de isometria e seu número máximo de supercargas.

\footnotetext{
${ }^{9}$ Uma discussão introdutória sobre essa construção e a relação entre constantes gravitacionais e comprimentos de Planck em várias dimensões pode ser encontrada em [13].

${ }^{10} \mathrm{Um}$ bom material pode ser encontrado em [22] e em suas referências.
} 
Para entender a origem das isometrias de $A d S_{5}$, basta analisar seus vetores de Killing. De acordo com a métrica dada em (6.20), as equações de Killing são:

$$
\begin{aligned}
\partial_{m} v_{n}+\partial_{n} v_{m} & =-2 \eta_{m n} \frac{v_{4}}{x^{4}}, \\
\partial_{m} v_{4}+\partial_{4} v_{m} & =2 \frac{v_{m}}{x^{4}}, \\
\partial_{4} v_{4} & =\frac{1}{x^{4}} v_{4} .
\end{aligned}
$$

Aqui, $v_{m}$ e $v_{4}$ são os vetores de Killing, sendo $u \equiv x^{4}$. Pela última equação, $v_{4}=\lambda\left(x^{m}\right) x^{4}$. Substituindo na primeira equação, obtem-se

$$
\partial_{m} v_{n}+\partial_{n} v_{m}=2 \eta_{m n} \lambda .
$$

Esse resultado é idêntico àquele do apêndice E sobre simetria conforme. É importante lembrar que todas as equações de Killing devem ser satisfeitas para uma isometria e, nesse caso, tudo fica mais evidente com os objetos na forma contravariante. Com isso, são identificadas imediatamente as isometrias da métrica $A d S_{5}$ : translações e transformações de Lorentz em $x^{m}$; dilatações e transformações especiais conformes em $x^{m}$ e $x^{4}$. Ou seja, o grupo de isometrias de é $S O(2,4)$.

Quanto à preservação de supersimetrias do espaço $A d S_{5}$, basta analisar as transformações de supersimetria do gravitino e do dilatino, dadas em (6.8). O obejtivo é encontrar restrições sobre o número de graus de liberdade dos espinores de Killing da transformação. Certamente, a transformação do dilatino não fornece informação alguma sobre os parâmetros de supersimetria e é identicamente satisfeita para a solução extrema da 3-brana. Entretanto, a transformação do gravitino pode impor alguma restrição sobre o número de graus de liberdade do parâmetro $\xi$. É possível mostrar através de uma condição de integrabilidade da equação do espinor de Killing que os espaços AdS admitem tantas supersimetrias quanto o espaço plano, de modo que não há restrições nas supercargas além do vínculo BPS [22].

$\mathrm{Na}$ próxima seção é estabelecida a conjectura de Maldacena.

\subsection{A Conjectura de Maldacena e o Dicionário da Correspondên- cia}

Até aqui, algumas semelhanças entre $\mathcal{N}=4 \mathrm{SYM}$ em $D=4$ e a solução extrema da 3-brana na supergravidade $I I$-B (no seu limite de validade, evidentemente) foram apontadas, mas nada feito formalmente. Essa abordagem tende a simplificar a evolução histórica das duas teorias, entretanto é suficiente para indicar aqui algumas evidências. Não constituem, em absoluto, uma prova da possível dualidade.

\subsubsection{Algumas Comparações}

Como é de praxe, as simetrias são uma excelente introdução. A tabela abaixo contém as simetrias globais das duas teorias.

\begin{tabular}{|c|c|c|}
\hline Simetria & $\mathcal{N}=4$ SYM em $D=4$ & Supergravidade $I I$-B em $A d S_{5} \times S^{5}$ \\
\hline \hline$S U(4) \sim S O(6)$ & simetria R & isometria de $S^{5}$ \\
\hline$S U(2,2) \sim S O(2,4)$ & simetria conforme & isometria de $A d S_{5}$ \\
\hline$S L(2, \mathbb{Z})$ & $(6.3)$ & $(6.9)$ \\
\hline$\#$ de supercargas de Poincaré & $16(\mathcal{N}=4$ Weyl $)$ & $16(1 / 2$ BPS, $\mathcal{N}=2$ Majorana-Weyl $)$ \\
\hline
\end{tabular}


É interessante o fato de que o balanço de simetrias é perfeito. É natural esperar que teorias duais possuam a mesma simetria. A razão disso é simples. Simetrias estão associadas a cargas conservadas e, naturalmente, ao descrever um modelo de duas formas diferentes, é essencial que as cargas conservadas em uma descrição sejam conservadas na outra, e vice-versa.

O grupo de simetria $S L(2, \mathbb{Z})$ merece um certo cuidado. Como foi apontado na seção anterior, o limite de validade clássico da solução extrema exige que $g_{s}<1$ e uma dualidade $\mathrm{S}$ pode retirar as soluções de tal regime. Além do mais, foi apontado que $S L(2, \mathbb{R})$ é o grupo de simetria no nível clássico. Isso será melhor entendido na próxima subseção, ao enunciar a conjectura nas suas formas forte e fraca.

Outro ponto que merece atenção em relação às duas teorias, são as suas expansões perturbativas. $\mathrm{Na}$ teoria de cordas, foi mostrado em (2.23) que a teoria em interação poderia ser entendida perturbativamente em uma série cujo parãmetro de acoplamento é o valor esperado do dílaton, organizado em potências de um invariante topológico, a característica de Euler de uma superfície. Coincidentemente, ao introduzir a expansão $1 / N$ em SYM, tal estrutura reaparece, e a organização da expansão é, para superfícies orientáveis, a mesma.

Prosseguindo a análise, é preciso aprofundar a relação entre a 3-brana extrema e a D3-brana. Por isso, entenda-se identificá-las. Ao fazê-lo, uma nova ponte entre as duas teorias é erguida.

A 3-brana extrema carrega uma carga de Ramond-Ramond $Q_{3}$ e é uma solução da supergravidade II-B. Em outras palavras, interage gravitacionalmente por intermédio das cordas fechadas (gráviton).

A D3-brana está fundamentalmente associada às cordas abertas. Como foi apontado na seção 2.8, quando $N$ D-branas são sobrepostas, as cordas que nela terminam podem ser associadas à cargas de Chan-Paton e, quando não orientáveis, a simetria de calibre $U(N)$ emerge naturalmente.

Como é frequentemente dito, a 3-brana extrema e a D3-brana podem ser descrições diferentes de um mesmo objeto. Como $\mathcal{N}=4 \mathrm{SYM}$ em $D=4$ pode ser obtida por uma redução dimensional de $\mathcal{N}=1$ SYM em $D=10$, construída a partir do espectro não-massivo das supercordas abertas na presença de $N$ D-branas, a identificação dessas duas branas é uma forte evidência da correspondência.

Essas e várias outras evidências, mais formais e incisivas, culminaram na conjectura de Maldacena, que será enunciada a seguir.

\subsubsection{A Conjectura e o Dicionário}

Em [29], é mostrado que o "limite de $N$ grande de certas teorias de campos conformes... incluem em seu espaço de Hilbert um setor que descreve a supergravidade em um produto de espaços Anti-deSitter, esferas e outras variedades compactas". Essa é conhecida como a forma fraca da conjectura de Maldacena. Em particular, o artigo original trata explicitamente de $\mathcal{N}=4 \mathrm{SYM}$ em $D=4(S U(N)$ é o grupo de calibre $^{11}$ ) e a supergravidade clássica no espaço $A d S_{5} \times S^{5}$.

Se a conjectura for válida, espera-se que observáveis nas duas teorias sejam, de alguma forma, relacionados. Logo após o trabalho de Maldacena [29], foi estabelecida a prescrição (dicionário) que mapeia as quantidades de relevância entre a teoria de calibre e a gravitacional [30]. O chamado dicionário da correspondência ainda não é completamente entendido e, ainda hoje, novas verificações são obtidas. Aqui, tendo introduzido algumas propriedades nas seções 6.1 e 6.2, o objetivo é bem mais modesto e apenas os mapeamentos mais evidentes são ilustrados.

Pelo dicionário da correspondência, o grau do grupo de calibre $N$ e a carga $Q_{3}$ da 3-brana devem ser iguais, $Q_{3} \equiv N$. Uma consequência disso, envolve o raio da geometria, $L$. Pela equação (6.18), este é relacionado ao grau do grupo de calibre, ao comprimento da corda e à constante de acoplamento $g_{s}$ por

$$
L^{4}=4 \pi l_{s}^{4} g_{s} N .
$$

\footnotetext{
${ }^{11} \mathrm{Na}$ subseção anterior o grupo de calibre é apontado como $U(N)$, mas no limite $N \rightarrow \infty$ as duas são, em primeira aproximação, idênticas.
} 
Outra entrada do dicionário que pode, sem maiores formalidades, ser introduzida é a associação entre as constantes de acoplamento dos dois lados da correspondência. Ao comparar (6.3) e (6.9), a identificação também é bastante objetiva: $4 \pi g_{s}=g_{Y M}^{2}$. Uma vez que $\lambda=g_{Y M}^{2} N, \lambda=4 \pi g_{s} N$. Ou seja, o parâmetro de t'Hooft, $\lambda$, está associado à constante de acoplamento da corda. Além desta, uma identificação análoga é feita entre o ângulo do dílaton e o campo da 0-forma $C_{0}$.

Através dessas observações, a forma fraca da conjectura de Maldacena também pode ser enunciada como:

A teoria de $\mathcal{N}=4 S Y M$ em $D=4$ e grupo de calibre $S U(N)$ no limite $N \rightarrow \infty$ e $\lambda$ grande é dual à teoria de supergravidade II-B clássica $\left(l_{s} \ll L\right.$ e $\left.g_{s} \ll 1\right)$ sobre o produto dos espaços $A d S_{5}$ e $S^{5}$ de mesmo raio $L$ e carga $N$ da 4-forma autodual.

Assim, a conjectura estabelece a equivalência das teorias nos limites dicutidos, sendo que o parâmetro de acoplamento da teoria de calibre, $\lambda$, é grande e a expansão perturbativa é realizada com suas potências negativas. Isso equivale, no lado gravitacional, a uma expansão em potências positivas do comprimento $l_{s}$. Aqui fica, de certa forma, escondido o problema mencionado anteriormente de que a correspondência é estabelecida para uma teoria de calibre $S U(N)$ enquanto, pela dualidade da 3-brana extrema e da D3-brana, o grupo de calibre é $U(N)$. Fato garantido pelo limite $N \rightarrow \infty$.

A conjectura foi, então, extensivamente estudada. Há, entretanto, dois inconvenientes na formulação descrita. A primeira delas envolve a identificação $Q_{3} \equiv N$. Essa condição, apesar de possível, não é natural na teoria clássica, uma vez que admite valores contínuos dos acoplamentos das branas com as formas. A segunda envolve a dualidade $\mathrm{S}$, que, na teoria clássica, fica mal definida, pois pode retirar uma solução do regime de validade clássico. Algum tempo depois, a conjecturada foi reformulada na que é conhecida como sua forma forte.

A teoria de $\mathcal{N}=4 S Y M$ em $D=4$ e grupo de calibre $S U(N)$ é dual a teoria de quântica de supercordas do tipo II-B em um campo de fundo $A d S_{5} \times S^{5}$, sendo $g_{Y M}^{2}=4 \pi g_{s}$ e $L^{4}=4 \pi l_{s}^{4} g_{s} N$.

Nesse enunciado, não há necessidade de se operar nos regimes perturbativos. Aqui, a simetria $S L(2, \mathbb{Z})$ passa a ser uma simetria completa da correspondência e a dualidade $S$, que mapeia regimes fortes e fracos, é bem definida. Mais sutil, entretanto, é que não existe restrição alguma sobre o grau do campo de calibre. O limite $N \rightarrow \infty$ não é mais um requisito, sendo necessário justificar a ausência do campo de calibre $U(1)$ em $U(N)=U(1) \otimes S U(N)$. Nesse caso, é possível mostrar que tal campo é desacoplado na dualidade $[22,30]$.

Há ainda um enunciado intermediário.

A teoria de $\mathcal{N}=4 S Y M$ em $D=4$ e grupo de calibre $S U(N)$ no limite de t'Hooft é dual a teoria clássica de supercordas do tipo II-B em um campo de fundo $A d S_{5} \times S^{5}$.

As identificações anteriores entre as constantes de ambas as teorias são as mesmas. A razão para um princípio intermediário está diretamente associada ao limitado entendimento da teoria de cordas em interação. Apesar dos avanços recentes, a teoria clássica é ainda muito mais acessível a possíveis cálculos e análises, o que faz a conjectura intermediária muito mais atraente.

O dicionário da correspondência é vasto e poderoso. Entretanto, qualquer descrição mais detalhada envolve ferramentas que não foram aqui apresentadas. Sua construção envolve, por exemplo, a associação da dimensão anômala de operadores na teoria de SYM com certos observáveis clássicos de soluções da supergravidade. Para citar outros exemplos, serão relembrados dois operadores introduzidos na subseção 6.1.3. É possível mostrar que o multipleto de supergravidade (6.6) é mapeado em excitações na teoria de 
supergravidade correspondendo à partículas de spin menor ou igual a 2. O multipleto de Konishi, dado em (6.5), corresponde a excitações massivas da supercorda $I I$-B [26, 30].

Uma das implicações mais profundas da correspondência AdS/CFT é que uma teoria de cordas vivendo em um espaço de 10 dimensões pode ser mapeada em uma teoria de calibre que vive na borda deste espaço, em 4 dimensões. Este é o chamado Princípio Holográfico. Sua relevância, como foi dito, está na afirmação de que toda a informação de uma teoria em um dado volume do espaço pode ser mapeada univocamente em sua superfície. Esse é um problema bastante fundamental e está presente em modelos cosmológicos e gravitacionais bastante modernos, como certos questionamentos sobre o horizonte de eventos de um buraco negro conter a informação "perdida" por ele absorvida.

\subsection{Comentários}

A correspondência AdS/CFT fala por si. É um dos grandes avanços da física teórica atualmente e, como muitos acreditam piamente, parece ser uma questão de tempo a sua prova absoluta. Como ferramenta de cálculo, tem sido utilizada extensivamente para estudar regimes altamente não triviais de teorias de calibre, inclusive nas chamadas descrições holográficas, em que sistemas idealizados hidrodinâmicos, supercondutores, entre outros, parecem ser descritos de forma mais conveniente pelo seu equivalente gravitacional.

Sua área de pesquisa é rica e envolve um profundo conhecimento da física teórica. De uma lado, a teoria de campos e suas várias facetas e desmembramentos quase sem fim, de outro, a teoria de cordas, principalmente sua descrição pelos formalismos de Green-Schwarz e espinores puros. Além da sua riqueza de conteúdos envolvidos, a correspondência AdS/CFT pode trazer uma nova interpretação dos fenômenos físicos. A principal possibilidade, envolve a grande teoria de unificação das forças da natureza, uma vez que possibilita a compreensão de fenômenos até hoje inacessíveis pelo modelos usuais, que funcionam perturbativamente. 


\section{Capítulo 7}

\section{Conclusão}

Efetuou-se aqui, uma apresentação parcial do desenvolvimento dos formalismos mais tradicionais da teoria de cordas. É evidente, entretanto, que esta vasta área de pesquisa não pode ser descrita nas rápidas palavras e demonstrações aqui apresentadas.

A corda bosônica foi incluída por motivos pedagógicos, correspondendo aos primeiros passos no estudo das cordas. Não é justo, entretanto, subestimar sua importância. Vários aspectos gerais da teoria supersimétrica (RNS, GS ou espinores puros) são muito melhor entendidos ao olhar para a corda bosônica. Por si só, a teoria já constitui uma área de pesquisa vasta e, por muito tempo, produtiva. Seus desenvolvimentos foram essenciais para atingir o estágio atual de compreensão das supercordas.

O formalismo de Ramond-Neveu-Schwarz foi descrito no texto de forma invertida em relação ao desenvolvimento histórico. Aqui a supersimetria foi um ingrediente, embora tenha sido produto da inclusão dos férmions na corda bosônica. Seu estudo levou à consideração da supersimetria no espaçotempo e ao posterior desenvolvimento do formalismo de Green-Schwarz.

A supercorda GS foi apresentada aqui em detalhes, especialmente a construção da sua ação, que não é uma generalização trivial da corda bosônica. O espectro da teoria foi obtido consistentemente e a supersimetria no espaço-tempo se mostrou fundamental para construir o espaço de Fock a partir de um único vácuo, descrevendo os estados bosônicos e fermiônicos em pé de igualdade.

A ação no limite de baixa energia das D-brana foi introduzida cuidadosamente, construindo as transformações de supersimetria e simetria kapa do campo de calibre e implementando essa construção na ação de Dirac-Born-Infeld. As ações de supergravidade foram indicadas, descrevendo seu conteúdo de campos e algumas de suas propriedades. Essas ações efetivas constituem a abordagem mais amigável para o entendimento da teoria em interação, especialmente em se tratando de teorias complexas como as supercordas.

Os primeiros capítulos foram construídos gradualmente para que a conjectura de Maldacena pudesse ser apresentada de forma conveniente, descrevendo as simetrias globais da teoria de calibre e da supergravidade, e apontando algumas de suas semelhanças. Embora descrita aqui brevemente, a chamada correspondência gravidade/calibre, permitiu vislumbrar pela primeira vez um resultado passível de teste (como os supercondutores holográficos), constituindo a ligação mais promissora entre a teoria de cordas e a natureza.

Todos os assuntos aqui discutidos consumiram um longo período de estudos e constituem uma formação mínima, porém sólida, para o prosseguimento das atividades de pesquisa a serem desenvolvidas no doutorado. Espera-se, no futuro, incorporar um melhor entendimento de todos os tópicos apresentados, incluindo o mais novo formalismo das supercordas, os espinores puros. 


\section{Apêndice A}

\section{A Dimensão do Espaço-Tempo}

Um dos resultados mais conhecidos da teoria de cordas é a "previsão" do número de dimensões que o espaço-tempo deve possuir para que a teoria quântica seja consistente. Há várias maneiras diferentes de averiguar tal resultado. Particularmente, a imposição da simetria de Lorentz (i.e. a álgebra dos geradores de Lorentz) no nível quântico é um critério muito elegante, embora sua demonstração seja extensa na abordagem escolhida [13].

A álgebra de Poincaré é dada em (C.1). Ao fazer a transição da teoria clássica para a teoria quântica escolhendo o calibre do cone de luz, é natural esperar uma quebra da simetria de Lorentz. De fato, verifica-se que

$$
\left[J^{-i}, J^{-j}\right] \neq 0
$$

a menos que a dimensão $D$ do espaço-tempo e a constante de ordenamento $a$, que fornece o desvio de massa, tenham valores bem definidos. Construindo o gerador para as cordas abertas no nível quântico obtem-se:

$$
J^{-i}=x^{-} p^{i}-\frac{1}{2 p^{+} l_{s}^{2}}\left[x^{i}\left(L_{0}^{T}-a\right)+\left(L_{0}^{T}-a\right) x^{i}\right]-\frac{i}{p^{+} l_{s}} \sum_{n=1}^{\infty} \frac{1}{n}\left(L_{-n}^{T} \alpha_{n}^{i}-\alpha_{-n}^{i} L_{n}^{T}\right) .
$$

A simetrização do termo entre colchetes advem do requisito de hermiticidade do operador.

Para mostrar a relação (A.1), algumas relações são úteis de antemão:

$$
\begin{array}{ccc}
{\left[x^{-}, p^{+}\right]=-i} & {\left[x^{i}, p^{j}\right]=i \eta^{i j}} & {\left[x^{i}, \alpha_{n}^{j}\right]=i l_{s} \eta^{i j} \delta_{n, 0}} \\
{\left[x^{i}, L_{m}^{T}\right]=i l_{s} \alpha_{m}^{i}} & {\left[\alpha_{m}^{i}, \alpha_{n}^{j}\right]=i m \eta^{i j} \delta_{m+n, 0}} & {\left[\alpha_{m}^{i}, L_{n}^{T}\right]=m \alpha_{m+n}^{i}} \\
& {\left[L_{n}^{T}, L_{m}^{T}\right]=(m-n) L_{m+n}^{T}+\left(\frac{D-2}{12}\right) m\left(m^{2}-1\right)} &
\end{array}
$$

As outras relações de comutação fundamentais são nulas. Todas as grandezas acima foram definidas no capítulo 2 .

Os cálculos ficam menos suscetíveis a erros se o problema for dividido em várias partes. Portanto, é interessante avaliar, como passo intermediário, os comutadores das grandezas $x^{-}, 1 / p^{+}, p^{i}, x^{i}, \alpha_{m}^{i}$ e $L_{m}^{T}$ com os geradores de Lorentz $J^{-j}$. O processo de obtenção é simples e os resultados são:

i. $\left[1 / p^{+}, J^{-j}\right]$

Nesse caso, apenas a relação $\left[x^{-}, p^{+}\right]$é utilizada. 


$$
\left[1 / p^{+}, J^{-j}\right]=-\frac{i p^{j}}{\left(p^{+}\right)^{2}}
$$

ii. $\left[p^{i}, J^{-j}\right]$

Como $\left[x^{i}, p^{j}\right]=i \eta^{i j}$ e esse é o único comutador relevante, obtem-se:

$$
\left[p^{i}, J^{-j}\right]=\frac{i}{p^{+} l_{s}^{2}} \eta^{i j}\left(L_{0}^{T}-a\right)
$$

iii. $\left[x^{i}, J^{-j}\right]$

$$
\left[x^{i}, J^{-j}\right]=i x^{-} \eta^{i j}-\frac{i}{2 p^{+}}\left(x^{j} p^{i}+p^{i} x^{j}\right)+\frac{1}{p^{+}} \sum_{n=1}^{\infty} \frac{1}{n}\left(\alpha_{-n}^{i} \alpha_{n}^{j}-\alpha_{-n}^{j} \alpha_{n}^{i}\right) .
$$

iv. $\left[x^{-}, J^{-j}\right]$

$$
\left[x^{-}, J^{-j}\right]=-\frac{i}{2\left(p^{+} l_{s}\right)^{2}}\left[x^{j}\left(L_{0}^{T}-a\right)+\left(L_{0}^{T}-a\right) x^{j}\right]+\frac{1}{\left(p^{+}\right)^{2} l_{s}} \sum_{n=1}^{\infty} \frac{1}{n}\left(L_{-n}^{T} \alpha_{n}^{j}-\alpha_{-n}^{j} L_{n}^{T}\right)
$$

v. $\left[\alpha_{m}^{i}, J^{-j}\right]$

Com um pouco mais de trabalho que os outros, obtem-se:

$$
\begin{aligned}
{\left[\alpha_{m}^{i}, J^{-j}\right]=-\frac{1}{2 p^{+} l_{s}^{2}} m\left(x^{j} \alpha_{m}^{i}+\alpha_{m}^{i} x^{j}\right)+\frac{i}{p^{+} l_{s}} \eta^{i j}\left(L_{0}^{T}-a\right) \delta_{m, 0} } & \\
& -\frac{i}{p^{+} l_{s}} \sum_{n=1}^{\infty} \frac{m}{n}\left(\alpha_{m-n}^{i} \alpha_{n}^{j}-\alpha_{-n}^{j} \alpha_{m+n}^{i}\right)-\frac{i}{p^{+} l_{s}} \eta^{i j} \sum_{n \neq 0} \frac{m}{n} L_{-n}^{T} \delta_{m+n, 0}
\end{aligned}
$$

vi. $\left[L_{m}^{T}, J^{-j}\right]$

$\mathrm{E}$, finalmente:

$$
\begin{array}{r}
{\left[L_{m}^{T}, J^{-j}\right]=-\frac{1}{p^{+} l_{s}^{2}}\left\{m\left(x^{j} L_{m}^{T}+L_{m}^{T} x^{j}\right)-i l_{s}\left[\alpha_{m}^{j}\left(L_{0}^{T}-a\right)+\left(L_{0}^{T}-a\right) \alpha_{m}^{j}\right]\right\}} \\
-\frac{i}{p^{+} l_{s}} \sum_{n=1}^{\infty} \frac{1}{n}\left[m\left(L_{m-n}^{T} \alpha_{n}^{j}-\alpha_{-n}^{j} L_{m+n}^{T}\right)+n\left(L_{m-n}^{T} \alpha_{n}^{j}+\alpha_{-n}^{j} L_{m+n}^{T}\right)-n\left(L_{-n}^{T} \alpha_{m+n}^{j}-\alpha_{m-n}^{j} L_{n}^{T}\right)\right] \\
-\frac{i}{p^{+} l_{s}} \sum_{n \neq 0} \frac{1}{n} \alpha_{n}^{j}\left(\frac{D-2}{12}\right) m\left(m^{2}-1\right) \delta_{m-n, 0} .
\end{array}
$$

Em particular, para $m=0$ :

$$
\left[L_{0}^{T}, J^{-j}\right]=\frac{i}{p^{+}} p^{j}\left(L_{0}^{T}-a\right) .
$$


Procedendo ao cálculo:

$$
\begin{aligned}
{\left[J^{-i}, J^{-j}\right] } & =x^{-}\left[p^{i}, J^{-j}\right]+\left[x^{-}, J^{-j}\right] p^{i} \\
& -\frac{1}{2 l_{s}^{2}}\left\{\frac{1}{p^{+}} x^{i}\left[L_{0}^{T}, J^{-j}\right]+\frac{1}{p^{+}}\left[x^{i}, J^{-j}\right]\left(L_{0}^{T}-a\right)+\left[\frac{1}{p^{+}}, J^{-j}\right] x^{i}\left(L_{0}^{T}-a\right)\right\} \\
& -\frac{1}{2 l_{s}^{2}}\left\{\frac{1}{p^{+}}\left(L_{0}^{T}-a\right)\left[x^{i}, J^{-j}\right]+\left[\frac{1}{p^{+}}, J^{-j}\right]\left(L_{0}^{T}-a\right) x^{i}+\frac{1}{p^{+}}\left[L_{0}^{T}, J^{-j}\right] x^{i}\right\} \\
& -\frac{i}{l_{s}} \sum_{m=1}^{\infty} \frac{1}{m}\left\{\frac{1}{p^{+}} L_{-m}^{T}\left[\alpha_{m}^{i}, J^{-j}\right]+\frac{1}{p^{+}}\left[L_{-m}^{T}, J^{-j}\right] \alpha_{m}^{i}+\left[\frac{1}{p^{+}}, J^{-j}\right] L_{-m}^{T} \alpha_{m}^{i}\right\} \\
& +\frac{i}{l_{s}} \sum_{m=1}^{\infty} \frac{1}{m}\left\{\frac{1}{p^{+}} \alpha_{-m}^{i}\left[L_{m}^{T}, J^{-j}\right]+\frac{1}{p^{+}}\left[\alpha_{-m}^{i}, J^{-j}\right] L_{m}^{T}+\left[\frac{1}{p^{+}}, J^{-j}\right] \alpha_{-m}^{i} L_{m}^{T}\right\}
\end{aligned}
$$

Substituindo as relações de comutação obtidas, reescreve-se $\left[J^{-i}, J^{-j}\right]=A^{i j}+B^{i j}+C^{i j}+D^{i j} . A^{i j}$ contem os termos proporcionais a $\eta^{i j}$. $B^{i j}$ contem os termos que envolvem produtos de $x^{i}, x^{j}, p^{i}$ e $p^{j}$. $C^{i j}$ contem os termos com apenas um somatório. $D^{i j}$ contem os somatórios duplos. Com algumas simplificações triviais:

$$
\begin{aligned}
A^{i j}= & \eta^{i j} \frac{1}{l_{s}^{2}}\left[i x^{-}\left(\frac{1}{p^{+}}\right)\left(L_{0}^{T}-a\right)-i\left(\frac{1}{p^{+}}\right) x^{-}\left(L_{0}^{T}-a\right)\right] \\
& -\eta^{i j}\left(\frac{1}{p^{+} l_{s}}\right)^{2} \sum_{m=1}^{\infty} \sum_{n \neq 0} \frac{1}{n}\left(L_{-n}^{T} L_{m}^{T} \delta_{n-m, 0}+L_{-m}^{T} L_{-n}^{T} \delta_{n+m, 0}\right)
\end{aligned}
$$




$$
\begin{aligned}
D^{i j}= & \sum_{m=1}^{\infty} \sum_{n=1}^{\infty} \frac{1}{n}\left(\frac{1}{p^{+} l_{s}}\right)^{2}\left\{L_{-m}^{T}\left(\alpha_{-n}^{j} \alpha_{m+n}^{i}-\alpha_{m-n}^{i} \alpha_{n}^{j}\right)\left(\alpha_{-n}^{j} \alpha_{-m+n}^{i}-\alpha_{-m-n}^{i} \alpha_{n}^{j}\right) L_{m}^{T}\right. \\
& \left.\left(L_{-m-n}^{T} \alpha_{n}^{j}-\alpha_{-n}^{j} L_{-m+n}^{T}\right) \alpha_{m}^{i} \alpha_{-m}^{i}\left(L_{m-n}^{T} \alpha_{n}^{j}-\alpha_{-n}^{j} L_{m+n}^{T}\right)\right\} \\
& +\sum_{m=1}^{\infty} \sum_{n=1}^{\infty} \frac{1}{m}\left(\frac{1}{p^{+} l_{s}}\right)^{2}\left\{L_{-n}^{T} \alpha_{n-m}^{j} \alpha_{m}^{i}+\alpha_{-m-n}^{j} L_{n}^{T} \alpha_{m}^{i}-L_{-m-n}^{T} \alpha_{n}^{j} \alpha_{m}^{i}+\alpha_{-n}^{j} L_{n-m}^{T} \alpha_{m}^{i}\right. \\
& \left.\quad+\alpha_{-m}^{i} L_{m-n}^{T} \alpha_{n}^{j}+\alpha_{-m}^{i} \alpha_{-n}^{j} L_{m+n}^{T}+\alpha_{-m}^{i} L_{-n}^{T} \alpha_{n+m}^{j}+\alpha_{-m}^{i} \alpha_{m-n}^{j} L_{n}^{T}\right\}
\end{aligned}
$$

As expressões acima não parecem nada promissoras, pois estão em sua forma bruta. Para um resultado mais palpável, é necessário certo trabalho para lapidá-las. O ponto mais importante, referente às expressões $C^{i j}$ e $D^{i j}$, é que o ordenamento normal deve ser levado em conta em todos os passos do processo.

Iniciando pelo termos mais simples:

$$
\begin{gathered}
A^{i j}=-\eta^{i j}\left(\frac{1}{p^{+} l_{s}}\right)^{2}\left(L_{0}^{T}-a\right) . \\
B^{i j}=\eta^{i j}\left(\frac{1}{p^{+} l_{s}}\right)^{2}\left(L_{0}^{T}-a\right) .
\end{gathered}
$$

Sua adição é nula, de modo que não contribuem para o resultado final.

Para $C^{i j}$ :

$$
\begin{aligned}
& C^{i j}=\frac{2}{\left(p^{+} l_{s}\right)^{2}} \sum_{m=1}^{\infty}\left(\alpha_{-m}^{i} \alpha_{m}^{j}-\alpha_{-m}^{j} \alpha_{m}^{i}\right)\left\{m\left(\frac{D-2}{24}\right)-\frac{1}{m}\left[\left(\frac{D-2}{24}\right)-a\right]\right\} \\
&+\frac{1}{\left(p^{+} l_{s}\right)^{2}} \sum_{m=1}^{\infty} \frac{1}{m}\left[\left(L_{-m}^{T} \alpha_{m}^{j}-\alpha_{-m}^{j} L_{m}^{T}\right) \alpha_{0}^{i}-\left(L_{-m}^{T} \alpha_{m}^{i}-\alpha_{-m}^{i} L_{m}^{T}\right) \alpha_{0}^{j}\right] \\
&-\frac{2}{\left(p^{+} l_{s}\right)^{2}} \sum_{m=1}^{\infty} \frac{1}{m}\left(\alpha_{-m}^{i} L_{0}^{T} \alpha_{m}^{j}-\alpha_{-m}^{j} L_{0}^{T} \alpha_{m}^{i}\right) \\
&-\frac{2}{\left(p^{+} l_{s}\right)^{2}} \sum_{m=1}^{\infty}\left(\alpha_{-m}^{i} \alpha_{m}^{j}-\alpha_{-m}^{j} \alpha_{m}^{i}\right) .
\end{aligned}
$$

$C^{i j}$ é antissimétrico e já está normalmente ordenado. Para confirmar, basta lembrar que $L_{m}^{T}$ comportase como um modo $\alpha_{m}^{i}$ no ordenamento. Ou seja: : $L_{m}^{T} \alpha_{n}^{i}:=L_{m}^{T} \alpha_{n}^{i}$ para $n>m$; e, para $n<m$, : $L_{m}^{T} \alpha_{n}^{i}:=\alpha_{n}^{i} L_{m}^{T}$.

Rearranjando $D^{i j}$ com o ordenamento explícito: 


$$
\begin{aligned}
& D^{i j}=\left(\frac{1}{p^{+} l_{s}}\right)^{2} \sum_{m=1}^{\infty} \sum_{n=1}^{m} \frac{1}{n}\left(\alpha_{-n}^{j} \alpha_{n-m}^{i} L_{m}^{T}-L_{-m}^{T} \alpha_{m-n}^{i} \alpha_{n}^{j}\right) \\
& -\left(\frac{1}{p^{+} l_{s}}\right)^{2} \sum_{m=1}^{\infty} \sum_{n=m+1}^{\infty} \frac{1}{n}\left(\alpha_{m-n}^{i} L_{-m}^{T} \alpha_{n}^{j}+\left[L_{-m}^{T}, \alpha_{m-n}^{i}\right] \alpha_{n}^{j}\right) \\
& +\left(\frac{1}{p^{+} l_{s}}\right)^{2} \sum_{m=1}^{\infty} \sum_{n=1}^{\infty} \frac{1}{n}\left(\alpha_{-n}^{j} L_{-m}^{T} \alpha_{m+n}^{i}+\left[L_{-m}^{T}, \alpha_{-n}^{j}\right] \alpha_{m+n}^{i}\right) \\
& -\left(\frac{1}{p^{+} l_{s}}\right)^{2} \sum_{m=1}^{\infty} \sum_{n=1}^{\infty} \frac{1}{n}\left(\alpha_{-m-n}^{i} L_{m}^{T} \alpha_{n}^{j}+\alpha_{-m-n}^{i}\left[\alpha_{n}^{j}, L_{m}^{T}\right]\right) \\
& +\left(\frac{1}{p^{+} l_{s}}\right)^{2} \sum_{m=1}^{\infty} \sum_{n=m+1}^{\infty} \frac{1}{n}\left(\alpha_{-n}^{j} L_{m}^{T} \alpha_{n-m}^{i}+\alpha_{-n}^{j}\left[\alpha_{n-m}^{i}, L_{m}^{T}\right]\right) \\
& +\left(\frac{1}{p^{+} l_{s}}\right)^{2} \sum_{m=1}^{\infty} \sum_{n=1}^{\infty} \frac{1}{n}\left(L_{-m-n}^{T} \alpha_{n}^{j} \alpha_{m}^{i}-\alpha_{-n}^{j} L_{n-m}^{T} \alpha_{m}^{i}\right) \\
& -\left(\frac{1}{p^{+} l_{s}}\right)^{2} \sum_{m=1}^{\infty} \sum_{n=1}^{\infty} \frac{1}{m}\left(L_{-m-n}^{T} \alpha_{n}^{j} \alpha_{m}^{i}+\alpha_{-n}^{j} L_{n-m}^{T} \alpha_{m}^{i}-\alpha_{-m-n}^{j} L_{n}^{T} \alpha_{m}^{i}\right) \\
& +\left(\frac{1}{p^{+} l_{s}}\right)^{2} \sum_{m=1}^{\infty} \sum_{n=1}^{\infty} \frac{1}{n}\left(\alpha_{-m}^{i} L_{m-n}^{T} \alpha_{n}^{j}-\alpha_{-m}^{i} \alpha_{-n}^{j} L_{n+m}^{T}\right) \\
& +\left(\frac{1}{p^{+} l_{s}}\right)^{2} \sum_{m=1}^{\infty} \sum_{n=1}^{\infty} \frac{1}{m}\left(\alpha_{-m}^{i} L_{m-n}^{T} \alpha_{n}^{j}+\alpha_{-m}^{i} \alpha_{-n}^{j} L_{n+m}^{T}-\alpha_{-m}^{i} L_{-n}^{T} \alpha_{m+n}^{j}\right) \\
& +\left(\frac{1}{p^{+} l_{s}}\right)^{2} \sum_{m=1}^{\infty} \sum_{n=1}^{m} \frac{1}{m}\left(\alpha_{n-m}^{j} L_{-n}^{T} \alpha_{m}^{i}+\left[L_{-n}^{T}, \alpha_{n-m}^{j}\right] \alpha_{m}^{i}\right) \\
& +\left(\frac{1}{p^{+} l_{s}}\right)^{2} \sum_{m=1}^{\infty} \sum_{n=m+1}^{\infty} \frac{1}{m}\left(L_{-n}^{T} \alpha_{n-m}^{j} \alpha_{m}^{i}-\alpha_{-m}^{i} \alpha_{m-n}^{j} L_{n}^{T}\right) \\
& -\left(\frac{1}{p^{+} l_{s}}\right)^{2} \sum_{m=1}^{\infty} \sum_{n=1}^{m} \frac{1}{m}\left(\alpha_{-m}^{i} L_{n}^{T} \alpha_{m-n}^{j}+\alpha_{-m}^{i}\left[\alpha_{m-n}^{j}, L_{n}^{T}\right]\right) \text {. }
\end{aligned}
$$

Nota-se que os limites do somatório foram arranjados de forma conveniente.

Após algumas manipulações:

$$
\begin{aligned}
D^{i j}=\frac{2}{\left(p^{+} l_{s}\right)^{2}} \sum_{m=1}^{\infty}( & m-1)\left(\alpha_{-m}^{j} \alpha_{m}^{i}-\alpha_{-m}^{i} \alpha_{m}^{j}\right) \\
& +\left(\frac{1}{p^{+} l_{s}}\right)^{2} \sum_{m \neq 0} \sum_{n \neq 0} \frac{1}{n}\left(: L_{m}^{T} \alpha_{n-m}^{i} \alpha_{-n}^{j}:-: L_{m+n}^{T} \alpha_{-m}^{i} \alpha_{-n}^{j}:\right) \\
& +\left(\frac{1}{p^{+} l_{s}}\right)^{2} \sum_{m \neq 0} \sum_{n \neq 0} \frac{1}{m}\left(: L_{m+n}^{T} \alpha_{-m}^{i} \alpha_{-n}^{j}:-: L_{n}^{T} \alpha_{-m}^{i} \alpha_{m-n}^{j}:\right) .
\end{aligned}
$$

Somando todas as contribuições para o cálculo do comutador: 


$$
\begin{aligned}
{\left[J^{-i}, J^{-j}\right] } & =A^{i j}+B^{i j}+C^{i j}+D^{i j} \\
& =\frac{2}{\left(p^{+} l_{s}\right)^{2}} \sum_{m=1}^{\infty}\left(\alpha_{-m}^{i} \alpha_{m}^{j}-\alpha_{-m}^{j} \alpha_{m}^{i}\right)\left\{m\left[\left(\frac{D-2}{24}\right)-1\right]-\frac{1}{m}\left[\left(\frac{D-2}{24}\right)-a\right]\right\} \\
& +\left(\frac{1}{p^{+} l_{s}}\right)^{2} \sum_{m \neq 0} \sum_{n} \frac{1}{m}\left(: L_{m+n}^{T} \alpha_{-m}^{i} \alpha_{-n}^{j}:+: L_{n}^{T} \alpha_{m-n}^{i} \alpha_{-m}^{j}:\right) \\
& -\left(\frac{1}{p^{+} l_{s}}\right)^{2} \sum_{m \neq 0} \sum_{n} \frac{1}{m}\left(: L_{m+n}^{T} \alpha_{-n}^{i} \alpha_{-m}^{j}:+: L_{n}^{T} \alpha_{-m}^{i} \alpha_{m-n}^{j}:\right)
\end{aligned}
$$

Os quatro últimos termos nos somatórios duplos anulam-se, basta fazer $n \rightarrow n-m$. Essa transformação é possível porque a soma é realizada sobre todo $n \in \mathbb{Z}$, contribuição que foi garantida por $C^{i j}$ para $n=0$ nos dois últimos termos da equação A.9. Portanto:

$$
\left[J^{-i}, J^{-j}\right]=\frac{2}{\left(p^{+} l_{s}\right)^{2}} \sum_{m=1}^{\infty}\left(\alpha_{-m}^{i} \alpha_{m}^{j}-\alpha_{-m}^{j} \alpha_{m}^{i}\right)\left\{m\left[\left(\frac{D-2}{24}\right)-1\right]-\frac{1}{m}\left[\left(\frac{D-2}{24}\right)-a\right]\right\}
$$

A condição $\left[J^{-i}, J^{-j}\right]=0$ implica, que para cada $m$ no somatório, a expressão se anule, sendo que os coeficientes de $m$ e $\frac{1}{m}$ devem fazê-lo independentemente. Assim, $\frac{D-2}{24}-1=0$ implica em $D=26$. A outra contribuição fornece $a=1$. Confirmando o resultado discutido na seção 2.6. 


\section{Apêndice B}

\section{Algumas Propriedades de Espinores em Várias Dimensões}

Neste apêndice são demonstradas algumas identidades envolvendo matrizes de Dirac e espinores. As derivações aqui mostradas são baseadas em $[4,7,14]$.

\section{B.1 Matrizes $\Gamma$}

Os objetos fundamentais dessa análise são as matrizes de Dirac $\Gamma^{\mu}$, definidas por:

$$
\left\{\begin{array}{l}
\Gamma^{\mu}, \operatorname{com} \mu=0,1, \ldots, D-1 \\
\left\{\Gamma^{\mu}, \Gamma^{\nu}\right\}=2 \eta^{\mu \nu} \\
\left(\Gamma^{\mu}\right)^{\dagger}=\Gamma^{0} \Gamma^{\mu} \Gamma^{0}
\end{array}\right.
$$

Todas as propriedades que não dependam da representação matricial devem ser baseadas somente em (B.1).

A representação matricial mínima de $\Gamma^{\mu}$ em $D$ dimensões é uma matriz quadrada de ordem $2^{\frac{[D]}{2}}$, em que $[D]$ é o maior inteiro par menor ou igual a $D$. Desse ponto em diante serão consideradas apenas dimensões pares, $D=2 p+2$, com $p=0,1,2, \ldots$.

Há uma matriz que requer atenção especial, a matriz de quiralidade $\Gamma^{D}$, que anticomuta com todas as matrizes de Dirac $\Gamma^{\mu}$ e é definida como:

$$
\Gamma^{D}=i^{x} \Gamma^{0} \Gamma^{1} \ldots \Gamma^{D-1} .
$$

A fase $i^{x}$ é facilmente determinada tendo em vista que $\Gamma^{D} \Gamma^{D}=\mathbb{I}$. Um cálculo direto fornece: $x=-p$. Portanto:

$$
\Gamma^{D}=i^{-p} \Gamma^{0} \Gamma^{1} \ldots \Gamma^{2 p+1}
$$

Para descrever uma matriz genérica $M$ na representação mínima, basta utilizar o conjunto das matrizes $\Gamma^{(n)}=\left\{\mathbb{I}, \Gamma^{\mu}, \Gamma^{\mu \nu}, \Gamma^{\mu \nu \rho} \ldots\right\}$, com $n=1,2, \ldots, 2^{D}$, em que $\Gamma^{\mu_{1} \mu_{2} \ldots \mu_{k}}$ é o produto antissimétrico de $k$ matrizes $\Gamma^{\mu}$, tal que:

i. $\Gamma^{(n)} \Gamma^{(n)}= \pm \mathbb{I}$ 
ii. Para uma dada $\Gamma^{(n)} \neq \mathbb{I}$ existe uma $\Gamma^{(m)}$, tal que $\left\{\Gamma^{(n)}, \Gamma^{(m)}\right\}=0$

iii. $\operatorname{Para} \Gamma^{(n)} \neq \mathbb{I}, \operatorname{tr}\left(\Gamma^{(n)}\right)=0$.

iv. O produto de duas matrizes $\Gamma$ também é uma matriz $\Gamma: \Gamma^{(n)} \Gamma^{(m)} \propto \Gamma^{(l)}$.

Todas as propriedades acima decorrem de (B.1).

Assim:

$$
M=\sum_{n=1}^{2^{D}} a_{n} \Gamma^{(n)},
$$

em que $a_{n}= \pm \operatorname{tr}\left(M \Gamma^{(n)}\right)$.

Uma identidade recorrente e bastante útil na determinação dos coeficientes $a_{n}$ é

$$
\Gamma^{\mu} \Gamma^{\mu_{1} \mu_{2} \ldots \mu_{k}} \Gamma_{\mu}=(-1)^{k}(D-2 k) \Gamma^{\mu_{1} \mu_{2} \ldots \mu_{k}} .
$$

Sua demonstração é bastante simples e o resultado é utilizado em alguns pontos da dissertação.

\section{B.2 Espinores de Majorana, Weyl e Majorana-Weyl}

Um dado espinor é dito espinor de Majorana se satisfaz à condição: $\psi=\psi^{C}$, em que o índice $C$ indica conjugação de carga, operação que será definida adiante.

A operação de conjugação de carga está intimamente ligada à conjugação complexa. Verifica-se, pelos lemas de Schur, que as matrizes $\Gamma^{\mu}$ e $\Gamma^{\mu *}$ devem estar relacionadas por uma transformação de similaridade:

$$
\Gamma^{\mu *}=B \Gamma^{\mu} B^{-1}
$$

Por recorrência, $B^{*} B= \pm \mathbb{I}$.

Dada a transformação de similaridade, o espinor $B^{*} \psi$ satisfaz à mesma equação de $\psi$ e a condição de Majorana é $B \psi=\psi^{*}$. Dessa forma, espinores de Majorana existem apenas para $B^{*} B=\mathbb{I}$ (para $B^{*} B=-\mathbb{I}, \psi$ é identicamente nulo).

Define-se a operação de conjugação de carga $C$ como:

$$
\psi^{C}=\bar{\psi} C \text {. }
$$

Para que $\psi^{C}$ satisfaça à equação de Dirac, verifica-se que a matriz de conjugação $C$ deve satisfazer à seguinte condição: $C \Gamma^{\mu T} C^{-1}=-\Gamma^{\mu}$ ( $T$ nesse caso indica transposição e não há no texto possibilidade de confusão com os geradores de Virasoro transversais, $\left.L_{m}^{T}\right)$.

A condição de Weyl refere-se à quiralidade do espinor. Espinores de Weyl são autoestados da matriz de quiralidade:

$$
\Gamma^{D} \psi= \pm \psi
$$

É importante observar que em dimensões de número ímpar, não é possível definir quiralidade, uma vez que são exauridas as possibilidades de construção de matrizes de Dirac.

A condição de Majorana-Weyl é muito mais restritiva, já que não é válida para espinores em qualquer dimensão, embora possa parecer uma simples extensão do que já foi discutido. Isso será demonstrado a seguir.

Como foi visto, $B^{*} B=\varepsilon \mathbb{I}, \operatorname{com} \varepsilon= \pm 1$. 


$$
\begin{aligned}
\Gamma^{\mu T} & =\left(\Gamma^{\mu \dagger}\right)^{*}=\left(\Gamma^{0} \Gamma^{\mu} \Gamma^{0}\right)^{*}=\left(B \Gamma^{0}\right) \Gamma^{\mu}\left(\Gamma^{0} B^{-1}\right) \\
& =\left(\Gamma^{\mu *}\right)^{\dagger}=\left(B \Gamma^{\mu} B^{-1}\right)^{\dagger}=\left[\left(B^{-1}\right)^{\dagger} \Gamma^{0}\right] \Gamma^{\mu}\left(\Gamma^{0} B^{\dagger}\right)
\end{aligned}
$$

Comparando as duas equações, $B=e^{i \alpha}\left(B^{\dagger}\right)^{-1}$, em que $\alpha \in \mathbb{R}$. Como $B^{\dagger} B=\lambda$ ( $\lambda$ é real e positivo) e $B$ é definido a menos de uma constante multiplicativa, escolhe-se

$$
B=\left(B^{\dagger}\right)^{-1} \Rightarrow B=\varepsilon B^{T} .
$$

Ou seja, $B$ é simétrico ou antissimétrico. Além disso, pela definição da matriz d conjugação de carga, $\left(B \Gamma^{0}\right) \Gamma^{\mu}\left(\Gamma^{0} B^{-1}\right)=-C^{-1} \Gamma^{\mu} C$, implicando que $C=-\Gamma^{0} B^{-1}$. Das condições acima, verifica-se que $C^{T}=-\varepsilon C$. Em outras palavras, quando $B$ é simétrico, $C$ é antissimétrico, e vice-versa. $\Gamma^{(n)}$ :

Com essas propriedades torna-se simples analisar a operação de conjugação de carga nas matrizes

$$
\Gamma^{(n)}(m)=(-1)^{m}(-1)^{\frac{1}{2} m(m-1)} C\left[\Gamma^{(n)}(m)\right]^{T} C^{-1},
$$

em que $m$ indica o número de matrizes $\Gamma^{\mu}$ que constituem $\Gamma^{(n)}$.

Portanto:

$$
\left[C^{-1} \Gamma^{(n)}(m)\right]=-\varepsilon(-1)^{\frac{1}{2} m(m-1)}\left[C^{-1} \Gamma^{(n)}(m)\right]^{T} .
$$

Uma vez que $\left\{\Gamma^{(n)}\right\}$ constitui uma base completa das matrizes quadradas de ordem $2^{\frac{D}{2}},\left\{C^{-1} \Gamma^{(n)}\right\}$ também é um conjunto completo. Como esses geradores tem simetria definida e há $\frac{1}{2} 2^{\frac{D}{2}}\left(2^{\frac{D}{2}}-1\right)$ matrizes antissimétricas em $G L\left(2^{\frac{D}{2}}\right), \varepsilon$ depende da dimensão $D$ do espaço-tempo. Contando o número de matrizes antissimétricas definidas pela identidade, deve-se obter que:

$$
\frac{1}{2} \sum_{m=0}^{D}\left[1-\varepsilon(-1)^{\frac{1}{2} m(m-1)}\right]\left(\begin{array}{l}
D \\
m
\end{array}\right)=\frac{1}{2} 2^{\frac{D}{2}}\left(2^{\frac{D}{2}}-1\right) .
$$

Reescrevendo a expressão:

$$
\varepsilon \sum_{m=0}^{D}(-1)^{\frac{1}{2} m(m-1)}\left(\begin{array}{l}
D \\
m
\end{array}\right)=2^{\frac{D}{2}}
$$

Como

$$
(-1)^{\frac{1}{2} m(m-1)}=\frac{1}{2}\left[(1+i) i^{m}+(1-i) i^{-m}\right]
$$

e

$$
\sum_{n=0}^{m} i^{n}\left(\begin{array}{c}
m \\
n
\end{array}\right)=2^{\frac{m}{2}} \exp \left(\frac{i m \pi}{4}\right)
$$

obtem-se:

$$
\varepsilon=-\sqrt{2} \cos \left(\frac{\pi}{4}(D+1)\right) .
$$

Portanto, espinores de Majorana $(\varepsilon=1)$ são definidos em $D=2,4(\bmod 8)$. 
Agora resta impor a condição de Weyl. Tomando o complexo conjugado da condição de Weyl:

$$
\begin{aligned}
\left(\Gamma^{D}\right)^{*} \psi^{*} & = \pm \psi^{*} \\
B^{-1}\left(\Gamma^{D}\right)^{*} B\left(B^{-1} \psi^{*}\right) & = \pm\left(B^{-1} \psi^{*}\right) \\
(-1)^{p} \Gamma^{D}\left(B^{-1} \psi^{*}\right) & = \pm\left(B^{-1} \psi^{*}\right) .
\end{aligned}
$$

Para $p$ par, $\psi$ e $B^{-1} \psi^{*}$ tem a mesma quiralidade e a representação de Weyl é dita autoadjunta. Para $p$ ímpar, a quiralidade é oposta e as representações de Weyl são adjuntas entre si.

Portanto a condição de Majorana-Weyl existe apenas para $D=2(\bmod 8)$.

Uma vez analisadas algumas destas propriedades, é natural questionar a existência de uma representação real das matrizes de Dirac $\Gamma^{\mu}$. Nesse caso $\left[B, \Gamma^{\mu}\right]=0$ para todo $\mu$, o que implica que $B=\mathbb{I}$. Para fazer isso é necessário procurar por uma transformação de similaridade adequada:

$$
\left(\Gamma^{\mu}\right)^{\prime}=A \Gamma^{\mu} A^{-1} \Rightarrow B^{\prime}=A^{*} B A^{-1} .
$$

Como foi visto, o objetivo é encontrar $B^{\prime}=\mathbb{I}$. Sabe-se que $B^{*} B=\mathbb{I}$ e $B=B^{T}$. Os autovalores de $B$ tem módulo unitário:

$$
B=\operatorname{diag}\left(e^{i \alpha_{1}}, e^{i \alpha_{2}}, \ldots\right) .
$$

Basta escolher

$$
A=\operatorname{diag}\left(e^{\frac{i \alpha_{1}}{2}}, e^{\frac{i \alpha_{2}}{2}}, \ldots\right),
$$

e obtem-se $B^{\prime}=\mathbb{I}$.

A representação em que $B=\mathbb{I}$ e $\Gamma^{\mu}$ é real é conhecida como representação de Majorana e possui as seguintes propriedades:

$$
\left\{\begin{array}{l}
C=-\Gamma^{0} \\
\Gamma^{0}=-\left(\Gamma^{0}\right)^{T} \\
\Gamma^{i}=\left(\Gamma^{i}\right)^{T} \\
\Gamma^{D}=\left(\Gamma^{D}\right)^{T} .
\end{array}\right.
$$

Além disso, quando espinores de Majorana-Weyl são considerados, observam-se duas identidades muito úteis:

$$
\begin{gathered}
\bar{\psi}_{A} \Gamma^{\mu_{1} \ldots \mu_{m}} \psi_{B}=(-1)^{\frac{m(m+1)}{2}} \bar{\psi}_{B} \Gamma^{\mu_{1} \ldots \mu_{m}} \psi_{A} . \\
\bar{\psi}_{A} \Gamma^{(n)}(m) \psi_{B}=(-1)^{m+1} \epsilon_{A} \epsilon_{B} \bar{\psi}_{A} \Gamma^{(n)}(m) \psi_{B} .
\end{gathered}
$$

Aqui, $\epsilon_{A}$ e $\epsilon_{B}$ são as quiralidades de $\psi_{A}$ e $\psi_{B}$, respectivamente. A primeira propriedade está associada à condição de Majorana e a segunda está associada à condição de Weyl.

A demonstração das identidades é bastante simples. Na primeira são utilizadas as relações de transposição na representação de Majorana e na segunda $\left\{\Gamma^{\mu}, \Gamma^{D}\right\}=0$. 


\section{B.3 Propriedade Especial}

Em um contexto supersimétrico, especialmente em $D=2,4$ e 10, existe uma propriedade especial, a saber,

$$
\Gamma_{\mu} \psi^{i} \bar{\psi}^{j} \Gamma^{\mu} \psi^{k} E_{i j k}=0 \text {. }
$$

Em que $E_{i j k}$ é objeto totalmente antissimétrico na troca dos índices $i, j$ e $k$.

No caso $D=10$ a propriedade acima é válida para espinores de Majorana-Weyl e pode ser demonstrada como segue.

Deixando os índices espinoriais explícitos:

$$
\begin{aligned}
E_{i j k}\left(\Gamma_{\mu}\right)_{A B} \psi_{B}^{i} \bar{\psi}_{C}^{j}\left(\Gamma^{\mu}\right)_{C D} \psi_{D}^{k}= & \\
& =E_{i j k} \psi_{B}^{i} \frac{1}{3}\left\{\left(\Gamma_{\mu}\right)_{A B}\left(\bar{\psi}^{j} \Gamma^{\mu} \psi^{k}\right)+\left(\bar{\psi}^{j} \Gamma_{\mu}\right)_{B}\left(\Gamma^{\mu} \psi^{k}\right)_{A}-\left(\bar{\psi}^{k} \Gamma_{\mu}\right)_{B}\left(\Gamma^{\mu} \psi^{j}\right)_{A}\right\}
\end{aligned}
$$

O termo entre chaves do lado direito da equação é uma matriz,

$$
M_{A B}=\left(\Gamma_{\mu}\right)_{A B}\left(\bar{\psi}^{j} \Gamma^{\mu} \psi^{k}\right)-\left(\Gamma^{\mu} \psi^{k}\right)_{A}\left(\bar{\psi}^{j} \Gamma^{\mu}\right)_{B}+\left(\Gamma^{\mu} \psi^{j}\right)_{A}\left(\bar{\psi}^{k} \Gamma^{\mu}\right)_{B},
$$

e, pelo teorema fundamental das matrizes de Dirac, pode ser expandido na base $\left\{\Gamma^{(n)}\right\}$ :

em que

$$
M_{A B}=\left(\bar{\psi}^{j} \Gamma^{\mu} \psi^{k}\right)\left(\Gamma_{\mu}\right)_{A B}+\sum_{n=1}^{2^{D}} a_{n} \Gamma_{A B}^{(n)},
$$

$$
a_{n}=\frac{\varepsilon_{n}}{2^{d}}\left[\bar{\psi}^{j} \Gamma_{\mu} \Gamma^{(n)} \Gamma^{\mu} \psi^{k}-\bar{\psi}^{k} \Gamma_{\mu} \Gamma^{(n)} \Gamma^{\mu} \psi^{j}\right]
$$

e $\epsilon_{n}$ é tal que $\left(\Gamma^{(n)}\right)^{2}=\varepsilon_{n} \mathbb{I}, \operatorname{com} \varepsilon_{n}= \pm 1$.

É possível restringir de forma considerável a análise dos coeficientes $a_{n}$ definidos acima. Em primeiro lugar, a antissimetria na troca $j \leftrightarrow k$ implica que $a_{n}=0$ para $\Gamma^{0} \Gamma^{(n)}$ antissimétrico. Pela identidade (B.5), apenas $\Gamma^{(n)}$ composto por um número ímpar de matrizes de Dirac contribui. Além disso, sabe-se que:

$$
\Gamma^{\mu_{1} \ldots \mu_{k}} \propto \Gamma^{\mu_{k+1} \ldots \mu_{D}} \Gamma^{D} .
$$

Portanto, apenas as matrizes $\Gamma^{(n)}(m)$ com $m \leq \frac{D}{2}=5$ precisam ser computadas.

Analisando cada termo separadamente:

i. $\Gamma^{(n)}=\Gamma_{\nu}$

Nesse caso $\varepsilon_{n}=+1$, portanto:

$$
a_{n} \equiv a_{\nu}=\frac{1}{32}\left[\bar{\psi}^{j} \Gamma_{\mu} \Gamma_{\nu} \Gamma^{\mu} \psi^{k}-\bar{\psi}^{k} \Gamma_{\mu} \Gamma_{\nu} \Gamma^{\mu} \psi^{j}\right]
$$

Pela identidade (B.3), obtem-se:

$$
a_{\nu}=-\frac{1}{4}\left[\bar{\psi}^{j} \Gamma_{\nu} \psi^{k}-\bar{\psi}^{k} \Gamma_{\nu} \psi^{j}\right]
$$

O resultado é equivalente, pelos argumentos acima, para $\Gamma^{(n)}$ composto por 9 matrizes de Dirac. 
ii. $\Gamma^{(n)}=\Gamma_{\mu \nu \rho}$

O produto $\Gamma^{0} \Gamma_{\mu \nu \rho}$ é antissimétrico, logo não contribui:

$$
a_{n} \equiv a_{\mu \nu \rho}=0
$$

Verificando:

- $\mu, \nu, \rho \neq 0$ :

$$
\left(\Gamma^{0} \Gamma_{\mu} \Gamma_{\nu} \Gamma_{\rho}\right)^{T}=-\Gamma_{\rho} \Gamma_{\nu} \Gamma_{\mu} \Gamma^{0}=\Gamma^{0} \Gamma_{\rho} \Gamma_{\nu} \Gamma_{\mu}=-\Gamma^{0} \Gamma_{\mu} \Gamma_{\nu} \Gamma_{\rho} .
$$

- $\mu=0$ :

$$
\left(\Gamma^{0} \Gamma_{0} \Gamma_{\nu} \Gamma_{\rho}\right)^{T}=\left(\Gamma_{\nu} \Gamma_{\rho}\right)^{T}=\Gamma_{\rho} \Gamma_{\nu}=-\Gamma_{\nu} \Gamma_{\rho}=-\Gamma^{0} \Gamma_{0} \Gamma_{\nu} \Gamma_{\rho}
$$

iii. $\Gamma^{(n)}=\Gamma_{\mu_{1} \mu_{2} \mu_{3} \mu_{4} \mu_{5}}$

Pela identidade (B.3),

$$
\Gamma^{\mu} \Gamma^{\mu_{1} \mu_{2} \ldots \mu_{k}} \Gamma_{\mu}=-(D-10) \Gamma^{\mu_{1} \mu_{2} \mu_{3} \mu_{4} \mu_{5}} .
$$

Logo, em $D=10$,

$$
a_{n} \equiv a_{\mu_{1} \mu_{2} \mu_{3} \mu_{4} \mu_{5}}=0 .
$$

Substituindo os resultados na expansão de $M$ :

$$
M_{A B}=\left(\bar{\psi}^{j} \Gamma^{\mu} \psi^{k}\right)\left(\Gamma_{\mu}\right)_{A B}-\frac{1}{4}\left(\Gamma_{\mu}\right)_{A B}\left[\bar{\psi}^{j} \Gamma^{\mu} \psi^{k}-\bar{\psi}^{k} \Gamma^{\mu} \psi^{j}+\bar{\psi}^{j} \Gamma^{\mu} \psi^{k}-\bar{\psi}^{k} \Gamma^{\mu} \psi^{j}\right]=0
$$

O que demonstra a propriedade:

$$
\Gamma_{\mu} \psi^{i} \bar{\psi}^{j} \Gamma^{\mu} \psi^{k} E_{i j k}=0 .
$$

No contexto de formas diferenciais, há duas conclusões importantes derivadas da propriedade acima:

i. A construção $\left(\chi \Gamma_{\mu} d \psi\right)\left(d \bar{\psi} \Gamma^{\mu} d \psi\right)$, em que fica implícito o produto exterior, é identicamente nula.

ii. Termos do tipo $\left(\chi \Gamma_{\mu} d \psi\right)\left(d \bar{\psi} \Gamma^{\mu} \psi\right)$ correspondem a uma derivada total quando $\chi$ é constante. Pela propriedade (B.6),

$$
2\left(\chi \Gamma_{\mu} d \psi\right)\left(d \bar{\psi} \Gamma^{\mu} \psi\right)+\left(\chi \Gamma_{\mu} \psi\right)\left(d \bar{\psi} \Gamma^{\mu} d \psi\right)=0 .
$$

Assim,

$$
3\left(\chi \Gamma_{\mu} d \psi\right)\left(d \bar{\psi} \Gamma^{\mu} \psi\right)+d\left[\left(\chi \Gamma_{\mu} \psi\right)\left(\bar{\psi} \Gamma^{\mu} d \psi\right)\right]=0,
$$

provando a afirmação. 


\section{Apêndice C}

\section{Supersimetria e Representações}

A construção da álgebra de supersimetria geralmente é descrita em $D=4$. Entretanto, ao introduzir a supersimetria no contexto de teoria de cordas, é necessário entender sua construção em dimensões arbitrárias. O objetivo do presente apêndice é introduzir essa construção e algumas de suas implicações nas teorias que dela fazem uso, como o vínculo BPS. Uma discussão bastante completa sobre o assunto pode ser encontrada em [28]. Para $D=4$ há muitas referências disponíveis e aqui foi seguida a notação de [19].

\section{C.1 Introdução}

O grupo de Poincaré inclui translações e transformações de Lorentz no espaço-tempo, representadas, respectivamente, pelos geradores $P^{\mu}$ e $J^{\mu \nu}$. A álgebra dos geradores é dada por:

$$
\begin{aligned}
{\left[P^{\mu}, P^{\nu}\right] } & =0 \\
{\left[J^{\mu \nu}, P^{\rho}\right] } & =\eta^{\mu \rho} P^{\nu}-\eta^{\nu \rho} P^{\mu} \\
{\left[J^{\mu \nu}, J^{\rho \sigma}\right] } & =\eta^{\mu \rho} J^{\nu \sigma}-\eta^{\mu \sigma} J^{\nu \rho}-\eta^{\nu \rho} J^{\mu \sigma}+\eta^{\nu \sigma} J^{\mu \rho} .
\end{aligned}
$$

A supersimetria pode ser vista como uma extensão das simetrias do espaço-tempo ao considerar geradores de caráter fermiônico. Desde o conhecido trabalho de Coleman e Mandula [20], acreditava-se que "todas as possíveis simetrias da matriz S" eram conhecidas. Entretanto, ao relaxar uma das condições do seu teorema e permitir uma álgebra de geradores anticomutantes, verificou-se a possibilidade de uma nova simetria, a supersimetria [21] (é importante lembrar que essa simetria já havia sido observada anteriormente em vários trabalhos independentes no começo da década de 70), dando origem a uma extensa e rica área de pesquisa em física teórica.

Os geradores de supersimetria são representados por $Q_{A}^{\mathcal{A}}$, sendo $\mathcal{A}=1, \ldots, \mathcal{N},(\mathcal{N}$ designa o número de supersimetrias) e $A$ o índice espinorial.

Para introduzir o assunto de forma gradual, a próxima seção trata da construção da álgebra de supersimetria em $D=2$, cujo interesse manifesta-se pelo estudo do formalismo RNS da supercorda. Na seção seguinte a construção é generalizada para dimensões arbitrárias, incluindo discussões particulares para os casos de interesse na dissertação, a saber $D=4,10,11$. 


\section{C.2 Supersimetria em $D=2$ e a Supercorda RNS}

Em duas dimensões, a álgebra de supersimetria apresenta sua primeira construção não trivial, uma vez que em uma dimensão não há geradores de Lorentz. Nesse caso, há os geradores de translação $P^{ \pm}=P^{0} \pm P^{1}$ e o gerador de Lorentz $J^{01} \equiv J$. Sua álgebra é resumida pelas seguintes relações:

$$
\begin{aligned}
{\left[P^{ \pm}, P^{ \pm}\right]=\left[P^{ \pm}, P^{\mp}\right]=[J, J] } & =0 \\
{\left[P^{ \pm}, J\right] } & = \pm P^{ \pm} .
\end{aligned}
$$

A última relação estabelece que $P^{ \pm}$possui peso \pm 1 em relação ao gerador de Lorentz e esse resultado é muito importante na construção da álgebra de supersimetria.

Para continuar, é conveniente utilizar a representação de Majorana. Nesse caso, os geradores de supersimetria são reais e as matrizes de Dirac bidimensionais são aquelas definidas na seção 3.2. Verificase, então, que as componentes dos espinores não se misturam por transformações de Lorentz:

$$
\mathcal{J}_{A B}=j\left(\Gamma_{0} \Gamma_{1}\right)_{A B}=\left(\begin{array}{cc}
j & 0 \\
0 & -j
\end{array}\right) .
$$

Aqui, $j$ é o parâmetro da transformação. Basicamente, a transformação de Lorentz corresponde a um reescalonamento dos espinores. Dessa forma, espera-se que

$$
\left[J, Q_{ \pm}^{\mathcal{A}}\right]=\mp k Q_{ \pm}^{\mathcal{A}}
$$

Aqui, $k \neq 0$ indica o peso dos geradores de supersimetria em relação ao gerador de Lorentz, sendo $Q_{ \pm}^{\mathcal{A}}$ as componentes de $Q^{\mathcal{A}}$. Desse fato, é evidente que uma álgebra de anticomutação entre os geradores $Q^{\mathcal{A}}$ deve possuir a seguinte estrutura:

$$
\begin{aligned}
\left\{Q_{ \pm}^{\mathcal{A}}, Q_{ \pm}^{\mathcal{B}}\right\} & =M^{\mathcal{A B}} P^{ \pm} \\
\left\{Q_{ \pm}^{\mathcal{A}}, Q_{\mp}^{\mathcal{B}}\right\} & =Z_{ \pm}^{\mathcal{A B}}+L^{\mathcal{A B}} J .
\end{aligned}
$$

$M^{\mathcal{A B}}$ e $L^{\mathcal{A B}}$ são coeficientes a serem determinados. $Z_{ \pm}^{\mathcal{A B}}=Z_{\mp}^{\mathcal{B} \mathcal{A}}$ é chamado de carga central, pois comuta com todos os geradores da álgebra de Poincaré e supersimetria. A álgebra de Poincaré implica que $L^{\mathcal{A B}}=0$, uma vez que a primeira relação definida acima comuta com todos os geradores de translação. Como essa é uma representação real dos geradores de supersimetria, os índices $\mathcal{A}$ podem ser associados a uma representação fundamental do grupo de rotação $S O(\mathcal{N})$ que deixa a álgebra invariante. Desse modo, a partir de uma rotação e um reescalonamento adequado dos geradores, a matriz $M$ pode ser feita igual à identidade e obtem-se a álgebra de supersimetria em duas dimensões:

$$
\begin{aligned}
& \left\{Q_{ \pm}^{\mathcal{A}}, Q_{ \pm}^{\mathcal{B}}\right\}=\delta^{\mathcal{A B}} P^{ \pm} \\
& \left\{Q_{ \pm}^{\mathcal{A}}, Q_{\mp}^{\mathcal{B}}\right\}=Z_{ \pm}^{\mathcal{A B}}
\end{aligned}
$$

Devido à primeira relação acima, nota-se que $k=\frac{1}{2}$.

Na supercorda RNS discutida no capítulo $3, \mathcal{N}^{2}=1$ e não há carga central. Verifica-se prontamente que as transformações de supersimetria descritas nesse capítulo satisfazem à algebra acima.

Uma possível generalização do formalismo RNS poderia incluir supersimetria estendida $\mathcal{N}>1$. O caso mais simples, $\mathcal{N}=2$, requer um espaço-tempo de dimensão crítica 2 , número bem menor que as 10 requeridas em $\mathcal{N}=1$ e muito limitado para qualquer interpretação física. Para $\mathcal{N}=4$ o caso é ainda mais esdrúxulo, requerendo uma dimensão crítica negativa. Isso é verificado pelo procedimento descrito no apêndice A generalizado para o caso supersimétrico. 


\section{C.3 Supersimetria em Dimensões Arbitrárias}

De forma geral, os geradores de supersimetria devem satisfazer à seguinte álgebra:

$$
\left\{Q_{A}^{\mathcal{A}}, \bar{Q}_{\mathcal{B} B}\right\}=\mathcal{M}^{\mathcal{A}}{ }_{\mathcal{B}} N_{A B}
$$

$\mathcal{M}_{\mathcal{B}}^{\mathcal{A}}$ são coeficientes a serem determinados e $N_{A B}$ é uma matriz construída a partir de uma combinação das matrizes de Dirac, contendo os geradores bosônicos.

O primeiro passo para entender a relação acima é analisar a comutação entre os geradores bosônicos e os geradores fermiônicos.

Como não há geradores de peso $\frac{3}{2}$ em relação aos geradores de Lorentz, espera-se que $\left[P^{\mu}, Q_{A}^{\mathcal{A}}\right]=0$. O raciocínio é muito parecido com o desenvolvido na seção anterior. Dessa forma, o único gerador bosônico que $N_{A B}$ pode conter é $P^{\mu}$, uma vez que $\left[J^{\mu \nu}, P^{\rho}\right] \neq 0$, e a única possibilidade de construção da relação acima é:

$$
\left\{Q_{A}^{\mathcal{A}}, \bar{Q}_{\mathcal{B} B}\right\}=M_{\mathcal{B}^{\mathcal{A}}}^{\mathcal{A}} P_{\mu}\left(\Gamma^{\mu} D\right)_{A B}+Z_{\mathcal{B}^{\mathcal{A}}} E_{A B}
$$

Novamente, o termo $Z^{\mathcal{A B}}$ é a carga central da álgebra e sua origem são possíveis cargas conservadas da teoria em questão. $D$ e $E$ são matrizes a serem determinadas.

Prosseguindo, os geradores de supersimetria são espinores de Lorentz, de modo que

$$
\left[J^{\mu \nu}, Q_{A}^{\mathcal{A}}\right]=\frac{1}{2} \Gamma_{A B}^{\mu \nu} Q_{B}^{\mathcal{A}}, \quad\left[J^{\mu \nu}, \bar{Q}_{\mathcal{A} A}\right]=-\frac{1}{2} \bar{Q}_{\mathcal{A B}} \Gamma_{B A}^{\mu \nu}
$$

Como os autovalores de $\Gamma^{\mu \nu}$ são unitários, a relação acima indica que os geradores de supersimetria têm peso $\pm \frac{1}{2}$ em relação aos geradores de Lorentz, de acordo com o que foi discutido para $D=2$. Verifica-se facilmente que

$$
\left[\Gamma^{\mu \nu}, D\right]=\left[\Gamma^{\mu \nu}, E\right]=0,
$$

basta analisar a comutação dos geradores de Lorentz com os dois lados de (C.3) e compará-los. Isso restringe absolutamente as matrizes $D$ e $E$. De fato, há apenas duas soluções covariantes para as equações acima: a matriz identidade $\mathbb{I}$ e a matriz de quiralidade $\Gamma^{D}$, definida apenas em dimensões pares. Esse resultado poderia ter sido antecipado, uma vez que qualquer outra combinação de matrizes $\Gamma$ requereria objetos tensoriais dos quais não se dispõem, como foi visto no apêndice B.

Na construção verifica-se que $M=M^{\dagger}, Z=-Z^{\dagger}$ e que a álgebra de supersimetria é invariante por transformações unitárias, sendo que $Q^{\mathcal{A}}$ pertence à representação fundamental e $\bar{Q}_{\mathcal{A}}$ à representação antifundamental do grupo $S U(\mathcal{N})_{R}$. Essa simetria é denominada simetria $R$. Utilizando-a, é possível diagonalizar a matriz $M$. Devido à sua hermiticidade, a positividade da energia $\left(P^{0}>0\right)$ implica na positividade de seus autovalores, de modo que uma transformação unitária e um reescalonamento adequado podem fazer $M$ igual à identidade. Assim, é obtida a álgebra de supersimetria em dimensões arbitrárias.

- $D$ par:

$$
\left\{Q^{\mathcal{A}}, \bar{Q}_{\mathcal{B}}\right\}=\delta_{\mathcal{B}}^{\mathcal{A}} P_{\mu} \Gamma^{\mu} f\left(\mathbb{I}, \Gamma^{D}\right)+Z_{\mathcal{B}}^{\mathcal{A}} g\left(\mathbb{I}, \Gamma^{D}\right) .
$$

Aqui, $f$ e $g$ são combinações lineares das matrizes $\mathbb{I}$ e $\Gamma^{D}$.

- D ímpar:

$$
\left\{Q^{\mathcal{A}}, \bar{Q}_{\mathcal{B}}\right\}=\delta_{\mathcal{B}}^{\mathcal{A}} P_{\mu} \Gamma^{\mu}+Z_{\mathcal{B}}^{\mathcal{A}}
$$

A implicação mais fundamental da álgebra acima é a igualdade de estados fermiônicos e bosônicos em uma teoria supersimétrica, argumento utilizado constantemente ao longo do texto. Para avaliar isso, é necessário definir o que são estados fermiônicos e bosônicos. Mais precisamente, o que os diferencia. 
Naturalmente, identificando a álgebra acima como uma álgebra de criação e destruição de spin (procedimento que será estudado em detalhes logo em seguida na construção das representações em $D=4$ ), verifica-se que um estado fermiônico difere por um número ímpar de operadores de criação $\bar{Q}$ de um estado bosônico. Define-se o operador $(-1)^{F}$, tal que

$$
\left\{Q,(-1)^{F}\right\}=0 \text {. }
$$

Assim,

$$
\operatorname{tr}\left[(-1)^{F}\left(\delta_{\mathcal{B}}^{\mathcal{A}} \Gamma^{\mu} P_{\mu}+Z_{\mathcal{B}}^{\mathcal{A}}\right)\right]=\operatorname{tr}\left[(-1)^{F}\left\{Q^{\mathcal{A}}, \bar{Q}_{\mathcal{B}}\right\}\right]=0
$$

pela propriedade de ciclicidade do traço. Supondo que todos os estados tenham um momento fixo igual e diferente de zero, obtem-se o resultado:

$$
\operatorname{tr}\left[(-1)^{F}\right]=0
$$

Isso indica que o número de estados bosônicos e fermiônicos é igual.

\section{C.3.1 Supersimetria em $D=4$ e suas Representações}

Em dimensões pares é possível utilizar uma base de Weyl, decompondo o espinor em componentes de quiralidade positiva e negativa,

$$
Q_{A}^{\mathcal{A}}=\left(\begin{array}{c}
Q_{\alpha}^{\mathcal{A}} \\
\tilde{Q}_{\dot{\alpha}}^{\mathcal{A}}
\end{array}\right)
$$

em que $Q_{\alpha}^{\mathcal{A}}$ e $\tilde{Q}_{\dot{\alpha}}^{\mathcal{A}}$ possuem quiralidades opostas. Para $D=4, \alpha, \dot{\alpha}=1,2$. Nesse caso, a álgebra pode ser dividida em duas partes que contêm toda a informação disposta em (C.3):

$$
\begin{aligned}
\left\{Q_{\alpha}^{\mathcal{A}},\left(\tilde{Q}_{\dot{\alpha}}^{\mathcal{B}}\right)^{\dagger} \tilde{\gamma}_{\dot{\alpha} \beta}^{0}\right\} & =Z^{\mathcal{A B}} \delta_{\alpha \beta}, \\
\left\{Q_{\alpha}^{\mathcal{A}}, \bar{Q}_{\mathcal{B} \dot{\alpha}}\right\} & =\delta_{\mathcal{B}}^{\mathcal{A}} P_{\mu} \gamma_{\alpha \dot{\alpha}}^{\mu}
\end{aligned}
$$

Aqui, as matrizes de Dirac escritas como

$$
\Gamma_{A B}^{\mu}=\left(\begin{array}{cc}
0 & \gamma_{\alpha \dot{\alpha}}^{\mu} \\
\tilde{\gamma}_{\dot{\alpha} \alpha}^{\mu} & 0
\end{array}\right),
$$

deixam evidente a questão da quiralidade.

Impondo a condição de Majorana (como foi visto no apêndice B) verifica-se que

$$
\left\{Q_{\alpha}^{\mathcal{A}}, Q_{\beta}^{\mathcal{B}}\right\}=Z^{\mathcal{A B}} C_{\alpha \beta}
$$

Aqui $C$ é a matriz de conjugação de carga, que é antissimétrica ${ }^{1}$. Isso implica que $Z^{\mathcal{A B}}$ também é antissimétrico na troca dos índices $\mathcal{A}$ e $\mathcal{B}$.

Desse modo a álgebra de supersimetria em $D=4$ é dada por:

$$
\begin{aligned}
\left\{Q_{\alpha}^{\mathcal{A}}, Q_{\beta}^{\mathcal{B}}\right\} & =Z^{\mathcal{A B}} \epsilon_{\alpha \beta}, \\
\left\{Q_{\alpha}^{\mathcal{A}}, \bar{Q}_{\mathcal{B} \dot{\alpha}}\right\} & =\delta_{\mathcal{B}}^{\mathcal{A}} P_{\mu} \gamma_{\alpha \dot{\alpha}}^{\mu} .
\end{aligned}
$$

\footnotetext{
${ }^{1}$ A necessidade da matriz de conjugação de carga vem de uma análise da comutação de geradores de Lorentz com geradores de supersimetria, resultado que decorre diretamente de (C.4).
} 
$\epsilon_{\alpha \beta}$ é o tensor antissimétrico em duas dimensões tal que $\epsilon_{12}=1$, sendo proporcional à matriz de conjugação de carga $C_{\alpha \beta}$.

Agora, é interessante analisar a representação da álgebra de supersimetria por estados de uma partícula.

\section{C.3.1.1 Representações Não Massivas}

Ao representar estados não massivos é útil fazer uma transformação de Lorentz de modo que o 4momento seja $P^{\mu}=(E, 0,0, E)$. Desse modo, identificando as matrizes $\gamma_{\alpha \dot{\alpha}}^{\mu}$ com as matrizes de Pauli,

$$
\gamma^{0}=-\tilde{\gamma}^{0}=\left(\begin{array}{ll}
1 & 0 \\
0 & 1
\end{array}\right), \quad \gamma^{1}=\tilde{\gamma}^{1}=\left(\begin{array}{ll}
0 & 1 \\
1 & 0
\end{array}\right), \quad \gamma^{2}=\tilde{\gamma}^{2}=\left(\begin{array}{cc}
0 & -i \\
i & 0
\end{array}\right), \quad \gamma^{3}=\tilde{\gamma}^{3}=\left(\begin{array}{cc}
1 & 0 \\
0 & -1
\end{array}\right)
$$

a álgebra de supersimetria assume a seguinte forma:

$$
\begin{aligned}
\left\{Q_{1}^{\mathcal{A}}, Q_{2}^{\mathcal{B}}\right\} & =Z^{\mathcal{A B}} \\
\left\{Q_{\alpha}^{\mathcal{A}},\left(Q_{\beta}^{\mathcal{B}}\right)^{\dagger}\right\} & =\delta_{\mathcal{B}}^{\mathcal{A}}\left(\begin{array}{cc}
2 E & 0 \\
0 & 0
\end{array}\right) .
\end{aligned}
$$

As relações acima definem uma álgebra de criação associada aos geradores $Q_{1}^{\mathcal{A}}$ e $\bar{Q}_{\mathcal{A} i}$. Ao considerar representações unitárias, verifica-se que $Q_{2}^{\mathcal{A}}=0$ e $Z^{\mathcal{A B}}=0$, consequência direta da álgebra. Esse raciocínio é estendido para mais dimensões na seção C.3.2. No referencial escolhido, o gerador $J^{12}$ comuta com o operador de momento, de modo que $\left[J^{12},\left\{Q_{1}^{\mathcal{A}}, \bar{Q}_{\mathcal{B} 1}\right\}\right]=0$. Assim, é possível associar $Q_{1}^{\mathcal{A}}$ e $\left(Q_{1}^{\mathcal{A}}\right)^{\dagger}$ a operadores que abaixam e levantam, respectivamente, a helicidade de um dado estado por $\frac{1}{2}$. Dado o estado $|h\rangle$, denominado vácuo de Clifford, com helicidade $h$, verifica-se que:

$$
\begin{aligned}
Q_{1}^{\mathcal{A}}|h\rangle & =0 \\
\left(Q_{1}^{\mathcal{A}}\right)^{\dagger}|h\rangle & \rightarrow \text { helicidade }\left(h+\frac{1}{2}\right) .
\end{aligned}
$$

O vácuo de Clifford é o estado de menor helicidade e é possível, a partir dele, construir $2^{\mathcal{N}}$ estados. A maior helicidade obtida é $h+\frac{\mathcal{N}}{2}$, com uma contribuição de cada supersimetria.

O caso de interesse em $D=4$ é $\mathcal{N}=4$ Super Yang-Mills. Partindo de um vácuo de Clifford de helicidade -1 , é construído o multipleto de calibre não massivo: campo de calibre $A_{\mu}$ com helicidade \pm 1 ; campos escalares reais $X^{i}$, com $i=1, \ldots, 6$; e espinores de Weyl $\lambda_{a}$, com $a=1, \ldots, 4$ e helicidade $\pm \frac{1}{2}$. Ao todo, são 16 graus de liberdade, 8 bosônicos e 8 fermiônicos.

\section{C.3.1.2 Representações Massivas e o vínculo BPS}

No caso de representações massivas, basta fazer uma transformação de Lorentz e colocar a partícula no referencial de repouso. Dessa forma, $P^{\mu}=(m, 0,0,0)$ e a álgebra de supersimetria é dada por:

$$
\begin{aligned}
\left\{Q_{\alpha}^{\mathcal{A}}, Q_{\beta}^{\mathcal{B}}\right\} & =Z^{\mathcal{A B}} \epsilon_{\alpha \beta}, \\
\left\{Q_{\alpha}^{\mathcal{A}},\left(Q_{\beta}^{\mathcal{B}}\right)^{\dagger}\right\} & =\delta_{\mathcal{B}}^{\mathcal{A}}\left(\begin{array}{cc}
m & 0 \\
0 & m
\end{array}\right) .
\end{aligned}
$$

Para uma carga central geral, a construção de uma álgebra de criação a partir das relações acima não é óbvia. 
Quando há apenas uma supersimetria o resultado é bastante simples. Em primeiro lugar, não há carga central, fato garantido por $Z^{\mathcal{A B}}=-Z^{\mathcal{B} \mathcal{A}}$. Dessa forma, são obtidas duas álgebras de criação, uma para cada componente dos espinores de Weyl. No caso massivo, a atuação dos geradores está associada à adição de momento angular. Dado um vácuo de Clifford $|s\rangle$ de spin s, os estados da representação são construídos a partir da atuação de $\left(Q_{\alpha}\right)^{\dagger}$ em $|s\rangle$. Como a construção $Q_{\alpha}^{\dagger} Q_{\beta}^{\dagger}$ é antissimétrica na troca dos índices de Weyl, sua atuação é equivalente a uma adiação de momento angular nulo. Desse modo, há duas situações possíveis:

- $s=0$. Há dois estados de $\operatorname{spin} 0,|0\rangle$ e $Q_{\alpha}^{\dagger} Q_{\beta}^{\dagger}|0\rangle$, e um estado de $\operatorname{spin} \frac{1}{2}, Q_{\alpha}^{\dagger}|s\rangle$.

- $s>0$. Além do vácuo de Clifford $|s\rangle$, há outro estado de spin $s$, um estado de $\operatorname{spin} s+\frac{1}{2}$ e um estado de $\operatorname{spin} s-\frac{1}{2}$.

Quando há superssimetria $\mathcal{N}=2$, a análise é um pouco mais trabalhosa, uma vez que a carga central pode ser diferente de zero.

$$
\begin{aligned}
\left\{Q_{\alpha}^{1}, Q_{\beta}^{2}\right\} & =Z \epsilon_{\alpha \beta}, \\
\left\{Q_{\alpha}^{\mathcal{A}},\left(Q_{\beta}^{\mathcal{B}}\right)^{\dagger}\right\} & =\delta_{\mathcal{B}}^{\mathcal{A}}\left(\begin{array}{cc}
m & 0 \\
0 & m
\end{array}\right) .
\end{aligned}
$$

Naturalmente, a simetria $R$ inclui mudanças de fases em cada um dos geradores de supersimetria e isso permite impor uma condição de realidade na carga central. Assim, desse ponto em diante $Z \in \mathbb{R}$. Multiplicando a primeira relação acima por $i \gamma_{\beta \dot{\alpha}}^{2}$, verifica-se imediatamente que:

$$
\left\{Q_{\alpha}^{1},\left(i Q_{\beta}^{2} \gamma_{\beta \dot{\alpha}}^{2}\right)\right\}=\left(\begin{array}{cc}
Z & 0 \\
0 & -Z
\end{array}\right) .
$$

Portanto, é possível definir $Q_{\alpha \pm}^{\dagger}=\left(Q_{\alpha}^{1}\right)^{\dagger} \pm i\left(Q^{2} \gamma^{2}\right)_{\alpha}$ de tal forma que toda a álgebra fica contida nas seguintes relações (a menos de fatores de normalização):

$$
\begin{aligned}
\left\{Q_{\alpha \pm}, Q_{\beta \mp}\right\} & =0 \\
\left\{Q_{\alpha \pm}, Q_{\beta \pm}^{\dagger}\right\} & =\left(\begin{array}{cc}
m \pm Z & 0 \\
0 & m \pm Z
\end{array}\right) .
\end{aligned}
$$

Aqui a álgebra de criação é identificada e a representação é construída de forma análoga ao que foi feito para uma supersimetria. Como o lado esquerdo da última relação acima é positivo definido para uma representação unitária, verifica-se que existe um vínculo sobre a carga central, a saber $|Z|<m$. De outro modo,

$$
\left\{Q_{\alpha}, Q_{\beta}^{\dagger}\right\} \leq 0
$$

para uma das componentes \pm e diz-se que a supersimetria é quebrada. Em outras palavras, a representação é construída a partir de apenas uma supersimetria e é chamada $\frac{1}{2}$ BPS. Esse vínculo é conhecido como vínculo BPS (Bogomolnyi-Prasad-Sommerfield).

A extensão para supersimetria $\mathcal{N}>2$ com carga central é bastante direta mas sua construção não é aqui relevante. Uma pequena discussão pode ser encontrada em [26] e nas referências ali contidas.

\section{C.3.2 Supersimetria e Supergravidade em $D=10,11$}

Existe um resultado muito bem conhecido em física de que campos em interação não massivos com spin maior que 2 em $D \geq 4$ violam a causalidade [31]. Tendo isso em mente, é possível estabelecer um 
limite para o número máximo de supercargas (geradores de supersimetria) que uma teoria consistente pode possuir. Basicamente, o critério de consistência é limitar o número de operadores que aumentam a helicidade de um dado estado construído a partir de um vácuo de Clifford. Dessa forma, de -2 até 2 são necessários 8 geradores. Assim, a álgebra de supersimetria pode ter no máximo 8 geradores que aumentam a helicidade de um estado. É correto afirmar, portanto, que o critério acima pode ser julgado pela representação espinorial dos geradores de supersimetria e, consequentemente, pela dimensão do espaço-tempo em questão.

É necessário entender a álgebra de supersimetria em várias dimensões e analisar as possibilidades coerentes. Como a discussão gira em torno de representações não massivas, a carga central será considerada nula (a motivação da escolha vem da discussão da supersimetria em $D=4$ ). A demonstração desse resultado é simples. De forma geral, a álgebra de supersimetria é dada em (C.4) e (C.5). Fazendo uma transformação de Lorentz, é possível escolher $P^{0}=P^{1} \neq 0$ e $P^{i}=0$ para $i=2, \ldots, D-1$. Desse modo,

$$
\left\{Q_{A}^{\mathcal{A}}, \bar{Q}_{\mathcal{B} B}\right\}=\delta_{\mathcal{B}^{\mathcal{A}}} P_{-} \Gamma_{A B}^{-}+Z^{\mathcal{A}}{ }_{\mathcal{B}} \delta_{A B}
$$

Naturalmente, um mesmo par de geradores de supersimetria não pode ter pesos diferentes em relação a um gerador de Lorentz específico. De forma conveniente, o gerador a ser usado como referência é $J^{01} \equiv J$, como foi feito ne seção C.2, o que define duas classes de relação mutuamente exclusivas:

$$
\begin{aligned}
\left\{Q_{A}^{\mathcal{A}}, \bar{Q}_{\mathcal{B} B}\right\} & =\delta_{\mathcal{B}}^{\mathcal{A}} P_{-} \Gamma_{A B}^{-}, \\
& \text {ou } \\
\left\{Q_{A}^{\mathcal{A}}, \bar{Q}_{\mathcal{B} B}\right\} & =Z^{\mathcal{A}}{ }_{\mathcal{B}} E_{A B} .
\end{aligned}
$$

Assim, cada par $(Q, \bar{Q})$ satisfaz a apenas uma das relações acima.

Como $\Gamma^{-}$é uma matriz singular, metade de seus autovalores são nulos. Diagonalizando-a, certamente metade dos pares de geradores conjugados satisfazem a $\left\{Q_{A}, \bar{Q}_{A}\right\}=0$, omitindo os índices de supersimetria. Em uma representação unitária, essa condição implica que $Q_{A}^{\mathcal{A}}=0$. Pela segunda relação, há apenas uma possbilidade de construção, $Z_{\mathcal{B}}^{\mathcal{A}_{\mathcal{B}}}=0$. O raciocínio pode ser estendido para todas as cargas centrais. Portanto, uma representação unitária não massiva de estados de uma partícula não admite carga central e sua álgebra de criação é construída a partir de

$$
\left\{Q_{A}^{\mathcal{A}},\left(Q_{B}^{\mathcal{B}}\right)^{\dagger}\right\}=\delta_{\mathcal{B}}^{\mathcal{A}} P_{\mu}\left(\Gamma^{\mu} \Gamma^{0}\right)_{A B}
$$

Resta contar o número de geradores que aumentam a helicidade. Seja $\mathcal{S}$ a dimensão da representação espinorial dos geradores de supersimetria $Q$ (ou seja, $\mathcal{S}$ é o número de supercargas). Por exemplo, um espinor de Majorana-Weyl em $D=10$ possui 16 graus de liberdade, ou seja, $\mathcal{S}=16$. Pela relação acima, metade dos geradores se anula, uma vez que $P_{\mu} \Gamma^{\mu}$ é singular. Do restante, metade é formada por operadores de criação, que aumentam a helicidade de um estado, e metade por operadores de destruição. Ou seja, apenas um quarto de $\mathcal{S}$ é constituída de operadores de criação de helicidade. Baseado no raciocínio desenvolvido no começo da seção,

$$
\frac{1}{4} \mathcal{S} \cdot \mathcal{N} \leq 8
$$

para que as representações não massivas contenham apenas partículas cujo módulo da helicidade seja menor ou igual a 2 .

Com a condição (C.8), é possível obter qual o tamanho máximo da representação espinorial dos geradores de supersimetria. É fácil ver que $\mathcal{S} \leq 32$ para supersimetria $\mathcal{N}=1$. Em $D=10$, que é o caso da supercorda, é possível chegar a esse limite de várias formas: $\mathcal{N}=1 \mathrm{com}$ a condição de Majorana ou com a condição de Weyl e $\mathcal{N}=2$ com a condição de Majorana-Weyl. Para $D=11$ não existe a condição de quiralidade, de modo que a única forma de construir uma representação não massiva é através da 
condição de Majorana, que fornece 32 cargas de supersimetria. Em $D=12$ há 128 graus de liberdade para um espinor de Dirac, de modo que seria necessário uma redução de $\frac{3}{4}$ para atingir 32 supercargas. Entretanto, a única condição em potencial (Majorana-Weyl) não é definida nesta dimensão, como foi visto no apêndice $B$. Portanto, $D=11$ é o número máximo de dimensões admitidas por uma teoria supersimétrica de partículas sem massa ${ }^{2}$.

A importância das representações não massivas é evidenciada no capítulo 5, na qual são introduzidas ações efetivas no limite de baixas energias. Como exemplo, um cálculo simples pode mostrar o número de graus de liberdade do conteúdo de campos da supergravidade $D=11$. Nesse caso são 8 operadores de criação. Por redução dimensional, essa álgebra de criação é equivalente à álgebra em $D=4 \operatorname{com} \mathcal{N}=8$, de modo que há $2^{8}=256$ estados, 128 bosônicos e 128 fermiônicos. O conteúdo de campos é descrito na subseção 5.3.1. Outro exemplo é a supercorda aberta, que possui supersimetria $\mathcal{N}=1$. Em termos de operadores de criação de helicidade, esta possui a metade do número da supercorda fechada. Portanto, há $2^{4}$ estados na representação da supercorda aberta, 8 bósons e 8 férmions.

\footnotetext{
${ }^{2}$ Mais precisamente, é possível defini-la em $D=12$ para teorias com dois tempos, mas isso não será explorado aqui.
} 


\section{Apêndice D}

\section{Matrizes Singulares e a Simetria kapa}

Neste apêndice, discute-se rapidamente algumas propriedades de matrizes singulares, inclusive a fixação do calibre $\Gamma^{+} \Theta^{\mathcal{A}}=0$ na supercorda GS.

\section{D.1 Matrizes Singulares}

As matrizes $\Gamma^{+}$e $\Gamma^{-}$são singulares. Condição implicada por $\left(\Gamma^{+}\right)^{2}=\left(\Gamma^{-}\right)^{2}=0$. Entretanto, sua soma não é singular, uma vez que $\left(\Gamma^{+}+\Gamma^{-}\right)^{2} \propto \mathbb{I}$. Apesar de sua simplicidade, servem ao propósito de caracterização das matrizes desse tipo.

Uma forma de estudar a atuação de matrizes singulares é através da obtenção de seus autovalores e autovetores. Como $\left(\Gamma^{+}\right)^{2}=0$, há ao menos um autovalor nulo. Por hipótese, $\Gamma^{+}$possui $d$ autovalores nulos. Dado um vetor $V$ desse espaço, é possível expandi-lo em uma base ortogonal que inclui os autovetores de autovalor nulo de $\Gamma^{+}$, designados por $V_{+}^{i}$ Assim,

$$
V=\sum_{i=1}^{d} a_{i} V_{+}^{i}+\sum_{i=1}^{32-d} b_{i} V_{-}^{i} .
$$

Como

$$
\Gamma^{+} V=\sum_{i=d+1}^{32} b_{i}\left(\Gamma^{+} V_{-}^{i}\right)
$$

e $\Gamma^{+}\left(\Gamma^{+} V\right)=0$, verifica-se que:

$$
\Gamma^{+} V_{-}^{i}=\sum_{j=1}^{d} c_{i j} V_{+}^{j} .
$$

A última relação implica que $\Gamma^{+}$possui a seguinte estrutura:

$$
\Gamma^{+}=\left(\begin{array}{cc}
0 & 0 \\
\sqrt{2} \mathbb{X} & 0_{d \times d}
\end{array}\right)_{32 \times 32} .
$$

O fator $\sqrt{2}$ foi colocado por conveniência futura.

A matriz $\Gamma^{-}$possui propriedades semelhantes à $\Gamma^{+}$, e $\Gamma^{-}=-\left(\Gamma^{+}\right)^{T}$, de modo que:

$$
\Gamma^{-}=\left(\begin{array}{cc}
0 & -\mathbb{X}^{T} \\
0 & 0_{d \times d}
\end{array}\right)_{32 \times 32} .
$$


Como $\left\{\Gamma^{+}, \Gamma^{-}\right\}=-2, d=16$ e $\mathbb{X}$ é uma matriz ortogonal. $\Gamma^{+}$e $\Gamma^{-}$possuem apenas 16 autovalores cada e todos são nulos.

Portanto, qualquer espinor pode ser expandido em uma base em que metade de suas componentes são autovetores de $\Gamma^{+}$, enquanto a outra metade é de autovetores de $\Gamma^{-}$.

\section{D.2 Simetria kapa}

Com o que foi desenvolvido na seção anterior, resta saber se a simetria kapa permite a escolha $\Gamma^{+} \Theta^{\mathcal{A}}=0$. Em outras palavras, supondo $\Theta^{\prime \mathcal{A}}(\tau, \sigma)$ tal que $\Gamma^{+} \Theta^{\prime \mathcal{A}} \neq 0$, é possível encontrar $\Theta^{\mathcal{A}}(\tau, \sigma)=$ $\Theta^{\prime \mathcal{A}}(\tau, \sigma)+\Pi_{\alpha} \cdot \Gamma \kappa^{\mathcal{A}}$ que satisfaça à condição de calibre $\Gamma^{+} \Theta^{\mathcal{A}}=0$ ? A resposta é afirmativa.

$$
\Gamma^{+} \Theta^{\mathcal{A}}=0 \Rightarrow \Gamma^{+} \Theta^{\prime \mathcal{A}}=-\Gamma^{+}\left(\gamma_{ \pm} \kappa^{\mathcal{A}}\right)
$$

Como foi visto, as matrizes $\gamma_{ \pm}$são singulares na concha de massa e $\left\{\Gamma^{+}, \gamma_{ \pm}\right\} \propto \mathbb{I}$.

$$
\begin{aligned}
\Theta^{\mathcal{A}} & =\sum_{i=1}^{16} a_{i} \psi_{+}^{i}+\sum_{i=1}^{16} b_{i} \psi_{-}^{i} \\
\kappa^{\mathcal{A}} & =\sum_{i=1}^{16} c_{i} \psi_{+}^{i}+\sum_{i=1}^{16} d_{i} \psi_{-}^{i} \\
\Gamma^{+} \Theta^{\mathcal{A}} & =\sum_{i=1}^{16} e_{i} \psi_{+}^{i} \\
\gamma_{ \pm} \kappa^{\mathcal{A}} & =\sum_{i=1}^{16} c_{i}\left(\gamma_{ \pm} \psi_{+}^{i}\right) \\
\Gamma^{+}\left(\gamma_{ \pm} \kappa^{\mathcal{A}}\right) & =\sum_{i=1}^{16} c_{i} \Gamma^{+}\left(\gamma_{ \pm} \psi_{+}^{i}\right) \\
& =\sum_{i=1}^{16} f_{i} \psi_{+}^{i} .
\end{aligned}
$$

Portanto, ao expandir o parâmetro da transformação kapa em uma base de autovetores de $\gamma_{ \pm}$e $\Gamma^{+}$, a condição acima evidencia que existe uma escolha de parâmetros que permite a fixação do calibre proposto. 


\section{Apêndice E}

\section{Simetria Conforme}

Antes de tudo, é importante entender o que significa a simetria conforme ou o que ela deixa invariante. Do ponto de vista de espaço-tempo, uma transformação conforme é um difeomorfismo que preserva a métrica a menos de uma fator local, ou seja, preserva o ângulo entre os vetores. Para uma transformação infinitesimal, $x^{\mu} \rightarrow x^{\mu}+v^{\mu}$, em que $v^{\mu}$ é o parâmetro da transformação, sabe-se que a métrica transformase como

$$
\delta g_{\mu \nu}=D_{\mu} v_{\nu}+D_{\nu} v_{\mu}
$$

Aqui, $D_{\mu}$ é a derivada covariante no espaço-tempo cuja métrica é $g_{\mu \nu}(x)$. Desse modo, uma transformação conforme pode ser representada infinitesimalmente por

$$
g_{\mu \nu}^{\prime}(x)=g_{\mu \nu}+\delta g_{\mu \nu}=[1+w(x)] g_{\mu \nu} .
$$

Tomando o traço da equação acima e substituindo a transformação da métrica, verifica-se que

$$
w(x)=\frac{2}{D} D_{\mu} v^{\mu} .
$$

Ou seja, uma transformação conforme infinitesimal de parâmetro $v^{\mu}$ deve satisfazer a

$$
D_{\mu} v_{\nu}+D_{\nu} v_{\mu}=\frac{2}{D} g_{\mu \nu} D_{\rho} v^{\rho} .
$$

Se a transformação é realizada no espaço plano, $g_{\mu \nu}(x)=\eta_{\mu \nu}$ e $D_{\mu}=\partial_{\mu}$, de modo que a equação acima é reduzida a

$$
\partial_{\mu} v_{\nu}+\partial_{\nu} v_{\mu}=\frac{2}{D} \eta_{\mu \nu} \partial_{\rho} v^{\rho}
$$

Para determinar suas soluções é conveniente fazer uma expansão de Taylor, tal que

$$
v^{\mu}(x)=a^{\mu}+c^{\mu \nu} x_{\nu}+c^{\mu \nu \rho} x_{\nu} x_{\rho}+\ldots
$$

Substituindo na equação diferencial (E.1) e comparando os dois lados da equação ordem a ordem em $x$ :

- ordem 0: $a^{\mu}$ é um parâmetro real qualquer, satisfazendo à equação trivialmente.

- ordem 1: verifica-se que

$$
c^{\mu \nu}+c^{\nu \mu}=\frac{2}{D} \eta^{\mu \nu} c_{\rho}^{\rho} .
$$

A igualdade admite duas soluções distintas:

$$
\begin{aligned}
c^{\mu \nu} & =\lambda \eta^{\mu \nu}, \\
c^{\mu \nu} & =-c^{\nu \mu} .
\end{aligned}
$$


- ordem $\geq 2$ : a equação dos parâmetros da forma $c^{\mu \nu \rho_{1} \ldots \rho_{n}}$ é

$$
\left(c_{\mu \nu \rho_{1} \ldots \rho_{n}}+c_{\nu \mu \rho_{1} \ldots \rho_{n}}\right)=\frac{2}{D} \eta_{\mu \nu}\left(\eta^{\lambda \sigma} c_{\lambda \sigma \rho_{1} \ldots \rho_{n}}\right) \equiv \frac{2}{D} \eta_{\mu \nu} C_{\rho_{1} \ldots \rho_{n}}
$$

Isso é facilmente verificável, lembrando que $c_{\mu \nu \rho_{1} \ldots \rho_{n}}$ é simétrico na troca de qualquer índice à exceção do primeiro. Observa-se que

$$
c_{\mu \nu \rho_{1} \ldots \rho_{n}}=\eta_{\mu \nu} C_{\rho_{1} \ldots \rho_{n}}+\sum_{k=1}^{n}\left[\left(\nu \leftrightarrow \rho_{k}\right)-\left(\mu \leftrightarrow \rho_{k}\right)\right]
$$

aparenta ser uma solução da equação algébrica (E.2). Os termos entre parênteses designam uma permutação de índices.

Apesar de (E.3) ser solução de (E.2), verifica-se que não satisfaz aos requisitos de simetria na permutação dos índices $\left(\nu \leftrightarrow \rho_{k}\right)$, à exceção do caso $n=1$, ou seja, segunda ordem. Outra razão para tal contribuição se anular é que sua existência requereria geradores de simetria com spin maior ou igual a 2 (a partir da terceira ordem). Isso pode ser visto da seguinte forma. Qualquer transformação dos campos é associada ao produto do gerador pelo parâmetro da transformação. Como os parâmetros $C_{\rho_{1} \ldots \rho_{n}}$ são totalmente simétricos, correspondem a operadores de spin n, por simples argumento de composição de índices vetoriais. Ou seja, não são bem vindos em uma teoria de calibre cujo campo de maior spin é 1 .

Portanto, a solução para as transformações conformes é dada por

$$
v^{\mu}(x)=\overbrace{a^{\mu}}^{\text {translação }}+\underbrace{\lambda x^{\mu}}_{\text {dilatação }}+\overbrace{c^{\mu \nu} x_{\nu}}^{\text {Lorentz }}+\underbrace{2 x^{\mu} c_{\rho} x^{\rho}-c^{\mu} x_{\rho} x^{\rho}}_{\text {conforme especial }} .
$$

Aqui, $a^{\mu}, \lambda, c^{\mu \nu}=-c^{\nu \mu}$ e $c^{\mu}$ são os parâmetros translação, dilatação, transformação de Lorentz e transformação conforme especial, respectivamente.

Naturalmente, o próximo passo é analisar os geradores das simetrias indicadas: $P^{\mu}$ (translação), $D$ (dilatação), $J^{\mu \nu}$ (Lorentz) e $K^{\mu}$ (conforme especial). Como a dimensão dos geradores é o inverso da dimensão dos parâmetros das transformações, verifica-se que

$$
\left[P^{\mu}\right]=1, \quad\left[K^{\mu}\right]=-1, \quad\left[J^{\mu \nu}\right]=[D]=0,
$$

em unidades de massa ${ }^{1}$. Dessa forma, a álgebra do grupo conforme é obtida sem maiores dificuldades. Além da álgebra de Poincaré, contém:

$$
\begin{aligned}
{\left[J^{\mu \nu}, K^{\rho}\right] } & =\eta^{\nu \rho} P^{\mu}-\eta^{\mu \rho} P^{\nu} \\
{\left[P^{\mu}, K^{\nu}\right] } & =2\left(J^{\mu \nu}+\eta^{\mu \nu} D\right) \\
{\left[D, P^{\mu}\right] } & =P^{\mu} \\
{\left[D, K^{\mu}\right] } & =-K^{\mu} \\
{\left[D, J^{\mu \nu}\right] } & =0 .
\end{aligned}
$$

Em $D=2$, a álgebra conforme é isomórfica à álgebra de Virasoro, de dimensão infinita. Nota-se claramente a semelhança entre a equação (E.1) e a equação de simetria residual na folha-mundo (2.13), o que justifica a denominação de calibre conforme no estudo da corda bosônica.

Para $D>2$ dimensões, a álgebra conforme é isomórfica àquela do grupo $S O(2, D)$ e isso é verificado diretamente a partir de um rearranjo conveniente de (C.1) e (E.5). Esse fato é bastante importante, pois permite a comparação entre as simetrias globais entre $\mathcal{N}=4 \mathrm{SYM}$ e a supergravidade $I I$-B, descrita no capítulo 6.

\footnotetext{
${ }^{1}$ Aqui é utilizado o sistema natural de unidades, em que $c=\hbar=1$.
} 


\section{Referências Bibliográficas}

[1] M. B. Green, J. H. Schwarz. Anomaly Cancellation in Supersymmetric D $=10$ Gauge Theory and Superstring Theory. Physics Letters B149, 1984. DOI:10.1016/0370-2693(84)91565-X.

[2] J. Polchinski. Dirichlet-Branes and Ramond-Ramond Charges. Physycal Review Letters, Volume 75, 1995. DOI: 10.1103/PhysRevLett.75.4724.

[3] K. Becker, M. Becker, J. H. Schwarz. String Theory and M-Theory: A Modern Introduction. Cambridge University Press, 2007.

[4] M. B. Green, J. H. Schwarz, E. Witten. Superstring Theory: Introduction. Cambridge Monographs on Mathematical Physics, 1988. Volume 1.

[5] J. G. Polchinski. String Theory: An Introduction to the Bosonic String. Cambridge Monographs on Mathematical Physics, 1998. Volume 1.

[6] E. Kiritsis. String Theory in a Nutshell. Princeton University Press, 2007.

[7] F. Gliozzi, J. Scherk and D. Olive. Supersymmetry, Supergravity Theories and the Dual Spinor Model. Nuclear Physics B, Volume 122 (1977), p. 253.

[8] M. B. Green, J. H. Schwarz. Covariant description of superstrings. Physics Letters B, Volume 136. DOI:10.1016/0370-2693(84)92021-5.

[9] J. G. Polchinski. String Theory: Superstring Theory and Beyond. Cambridge Monographs on Mathematical Physics, 1998. Volume 2.

[10] W. Siegel. Hidden Local Supersymmetry in the Supersymmetric Particle Action. Physics Letters B, Volume 128, 1983. DOI: 10.1016/0370-2693(83)90924-3.

[11] L. Brink, John H. Schwarz. Quantum superspace. Physics Letters B, Volume 100. DOI: 10.1016/03702693(81)90093-9.

[12] N. Berkovits. Super-Poincaré Covariant Quantization of the Superstring. JHEP 0004 (2000) 018. DOI: $10.1088 / 1126-6708 / 2008 / 01 / 065$. (arXiv:0712.0324v2 [hep-th])

[13] B. Zwiebach. First Course in String Theory. Cambridge University Press, 2004.

[14] R. H. Good, Jr. Properties of the Dirac Matrices. Reviews of Modern Physics 27 (1955), p. 187. DOI: 10.1103/RevModPhys.27.187

[15] M. Aganagic, C. Popescu, J. H. Schwarz. D-brane actions with local kapa symmetry. Physics Letters B, Volume 393. DOI: 10.1016/S0370-2693(96)01643-7. (arXiv:hep-th/9610249v2) 
[16] M. Aganagic, C. Popescu, J. H. Schwarz. Gauge-invariant and gauge-fixed D-brane actions. Nuclear Physics B, Volume 495. DOI: 10.1016/S0550-3213(97)00180-6. (arXiv:hep-th/9612080v3)

[17] E. Cremmer, B. Julia, J. Scherk. Supergravity Theory in 11 Dimensions. Physics Letters B, Volume 76 (1978). DOI:10.1016/0370-2693(78)90894-8.

[18] D. Z. Freedman, P. van Nieuwenhuizen, S. Ferrara. Progress toward a Theory of Supergravity. Physical Review D 13, 3214 (1976).DOI: 10.1103/PhysRevD.13.3214.

[19] J. Wess, J. Bagger. Supersymmetry and Supergravity. Princeton University Press, 1992. $2^{\text {a }}$ edição revisada.

[20] S. Coleman, J. Mandula. All Possible Symmetries of the S Matrix. Physical Review, Volume 159 (1967). DOI: 10.1103/PhysRev.159.1251.

[21] R. Haag, J. T. Lopuszanski, M. Sohnius. All Possible Generators of Supersymmetries of the S-matrix. Nuclear Physics B, Volume 88, 1975. DOI: 10.1016/0550-3213(75)90279-5.

[22] O. Aharony, S. S. Gubser, J. Maldacena, H. Ooguri, Y. Oz. Large N Field Theories, String Theory and Gravity. Physics Reports, Volume 323, 2000. DOI: 10.1016/S0370-1573(99)00083-6.

[23] G. t Hooft. A Planar Diagram Theory for Strong Interactions. Nuclear Physics B, Volume 72. DOI: 10.1016/0550-3213(74)90154-0.

[24] S. Coleman. 1/N. SLAC-PUB-2484. The International School of Subnuclear Physics: Pointlike Structures Inside and Outside Hadrons. Erice Lectures, 1979.

[25] J. H. Schwarz, P. C. West. Symmetries and Transformations of Chiral $\mathcal{N}=2, D=10$ Supergravity. Physics Letters B, Volume 126, 1983. DOI: 10.1016/0370-2693(83)90168-5.

[26] E. D'Hoker, D. Z. Freedman. Supersymmetric Gauge Theories and the AdS/CFT Correspondence. arXiv:hep-th/0201253v2.

[27] L. I. Bevilaqua. Branas em Supergravidade. Dissertação de Mestrado. Universidade de São Paulo, IF-DFMA. USP/IF/SBI-026/2006. Endereço digital: www.teses.usp.br/teses/disponiveis/ 43/43134/tde-29032007-211600/publico/Tese.pdf.

[28] S. Weinberg. The Quantum Theory of Fields: Supersymmetry. Cambridge University Press. 2005. Volume 3.

[29] J. M. Maldacena. The Large N Limit of Superconformal Field Theories and Supergravity. Advances in Theoretical and Mathematical Physics, Volume 2, 1998. [arXiv:hep-th/9711200].

[30] E. Witten. Anti-deSitter Space And Holography. Advances in Theoretical and Mathematical Physics, Volume 2, 1998.[arXiv:hep-th/9802150v2]

[31] S. Weinberg, E. Witten. Limits on Massless Particles. Physics Letters B, Volume 96, 1980. DOI: 10.1016/0370-2693(80)90212-9. 